\title{
CONTRIBUCIONES AL ESTABLECIMIENTO DE UNA RED GLOBAL DE SENSORES INALAMBRICOS INTERCONECTADOS
}

\author{
Eduardo Omar Sosa
}

Director: Stefan Fischer

Codirector: Francisco Javier Díaz

Tesis presentada para obtener el grado de Doctor en Ciencias Informáticas

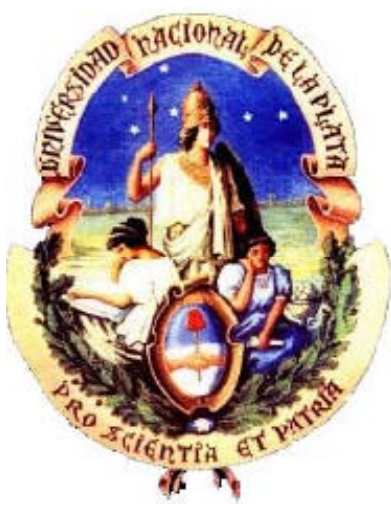

Facultad de Informática - Universidad Nacional de La Plata 


\begin{abstract}
A mi familia, por haber sido soportes $y$ principales inversores en tiempo, comprensión $y$ acompañamiento durante el presente proyecto.
\end{abstract}




\section{Agradecimientos}

A los profesores Stefan Fischer y Francisco Javier Díaz, por su constante apoyo y asesoramiento.

A las autoridades del Rectorado, y de la Facultad de Ciencias Exactas, Químicas y Naturales de la Universidad Nacional de Misiones quienes apoyaron entusiastamente este proyecto

Al personal de Laboratorio de Nuevas Tecnologías Informáticas de la Universidad de La Plata.

A los integrantes del Instituto de Telemática de la Universidad de Lübeck, por su predisposición, amabilidad y colaboración continúa.

A mis colegas de la cátedra en la Facultad de Ciencias Exactas, Químicas y Naturales de la Universidad Nacional de Misiones quienes me apoyaron en todo momento, cubriéndome en mis actividades cuando debí ausentarme para asistir a La Plata ó a Lübeck.

Al Dr. Carlos E. Galián y a través suyo al Parque Tecnológico Misiones PTMi. 
"The most profound technologies are those that disappear. They weave themselves into the fabric of everyday life until they are indistinguishable fram it."

"Las tecnologías más significativas son aquellas que se mimetizan entretejiéndose en la trama de la vida catidiana hasta que se tornan indistinguibles de la vida misma.

Mark Weiser, 1991 


\section{Índice General}

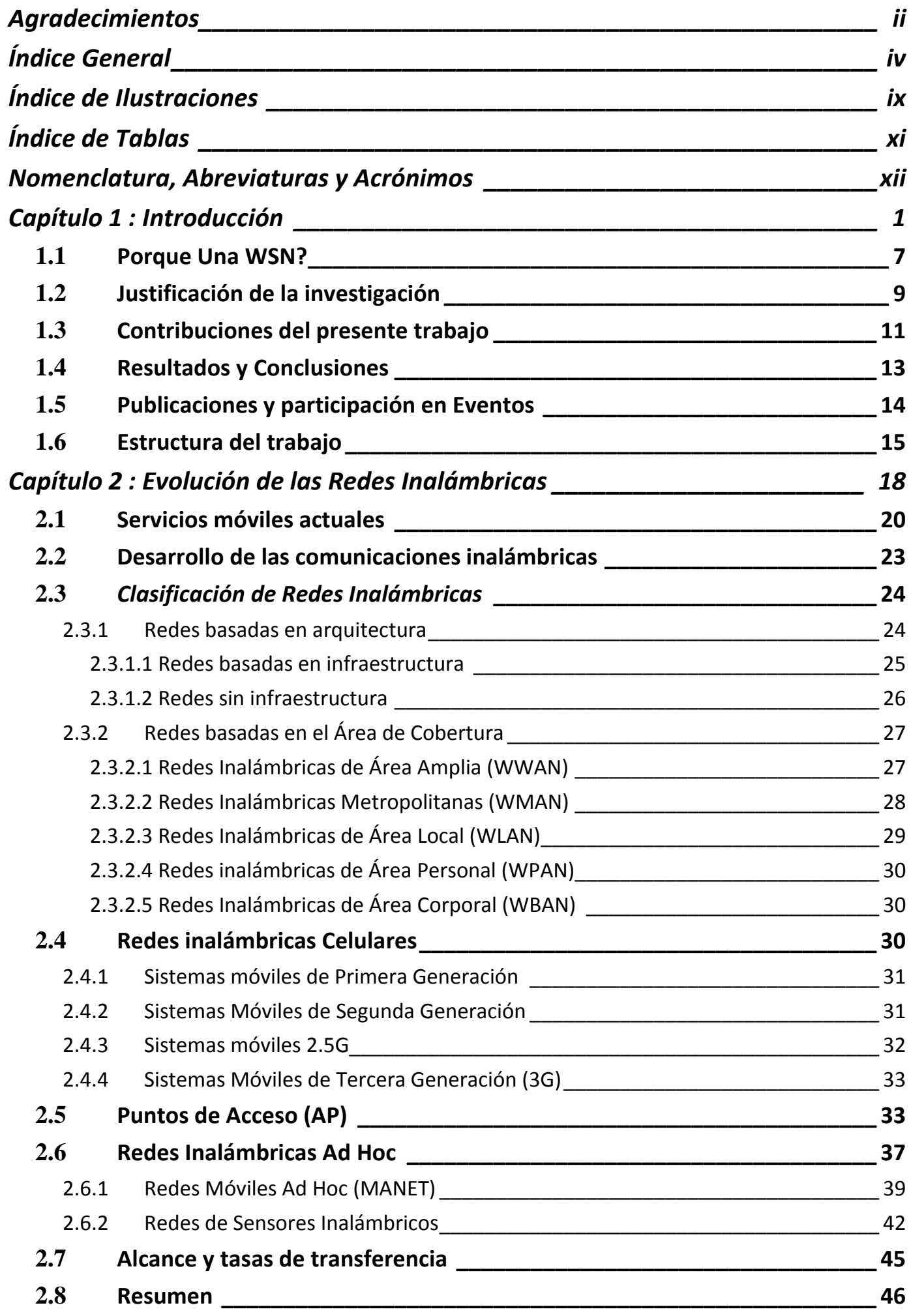


Capítulo 3 : Redes de Sensores Inalámbricos

3.1 El Desarrollo Tecnológico y la escala

3.2 Características de las WSN _ 56

3.3 Escenarios de Aplicación __ 56

3.4 Factores de diseño de las WSN _ 63

$3.5 \quad$ Métricas de evaluación de las WSN___ 63

3.5.1 Vida útil__ 64

3.5.2 Cobertura _ 66

3.5.3 Tolerancia a fallas _ 69

3.5.4 Costo y facilidad de instalación___ 70

3.5.5 Tiempo de respuesta___ 72

3.5.6 Precisión Temporal __ 72

3.5.7 Privacidad _ 73

3.5.8 Seguridad__ 73

3.5.9 Tasa Efectiva de Muestreo __ 75

3.6 Componentes de Hardware _ 76

3.7 Métricas de evaluación de nodos individuales __ 79

3.8 Energía___ 79

3.8.1 Flexibilidad — 80

3.8.2 Robustez — 80

3.8.3 Seguridad___ 81

3.8.4 Comunicación __ 82

3.8.5 Capacidad de Cálculo __ 83

3.8.6 Sincronización __ 83

3.8.7 Tamaño y costo __ 84

3.9 Prestaciones del Hardware_ 85

3.9.1 Energía__ 85

3.9.1.1 Tecnología de las baterías __ 87

3.9.1.2 Regulación de Voltaje ___ 89

3.9.1.3 Energías renovables __ 89

3.9.2 Radio 93

3.9.2.1 Alcance de transmisión___ 95

3.9.2.2 Modulación y Tasa de Transferencia ___ 96

3.9.2.3 Tiempo de encendido y "power-aware" __ 96

3.10 Controladores 98

3.10.1 Microcontroladores _ 100

3.11 Memoria 105

3.12 Equipo de Comunicaciones_ 105

3.12.1 Elección del medio de Comunicación __ 105

3.12.2 Transceptores__ 106

3.13 Resumen 109 
4.1 Estructura de las redes de sensores__ 111

4.2 Roles de los nodos en las WSN___ 113

4.3 Múltiples orígenes y sumideros __ 116

4.4 WSN estáticas y móviles___ 117

4.5 Movilidad__ 117

4.6 Objetivos de optimización __ 118

4.6.1 Calidad de servicio _ 120

4.6.1.1 Heterogeneidad _ 123

4.6.1.2 Eficiencia energética___ 123

4.6.1.3 Precisión temporal __ 124

4.6.1.4 Escalabilidad __ 124

4.6.1.5 Relación costo-beneficio _ 125

4.6.1.6 Confiabilidad___ 125

4.6.1.7 Robustez _ 126

4.6.1.8 Movilidad _ 126

4.6.1.9 Seguridad _ 127

4.7 Principios de diseño de WSNs _ 127

4.8 Centralidad de datos_ 130

4.9 Otros fundamentos de diseño de WSN _ 132

4.10 Optimización de la pila de protocolos __ 133

4.11 Interfaces de servicio en WSN __ 135

4.12 Resumen _ 137

Capítulo 5 : Las simulaciones__ 139

5.1 Desafíos de la simulación ___ 141

5.2 Ventajas de la simulación___ 144

5.3 Datos a simular 145

5.4 Simulación en WSN _ 146

5.5 Simuladores comunes en WSN _ 147

5.5.1 Network Simulator 2 (NS-2) __ 148

5.5.2 SensorSim _ 149

5.5.3 Prowler and JProwler 150

5.5.4 OMNeT++ — 150

5.5.5 GTSNetS__ 150

5.5.6 J-Sim — 151

5.5.7 SENS 151

5.5.8 SENSE_ 151

5.5.9 TOSSIM _ 152

5.5.10 Shawn 152

5.6 Selección del simulador _ـ 153 


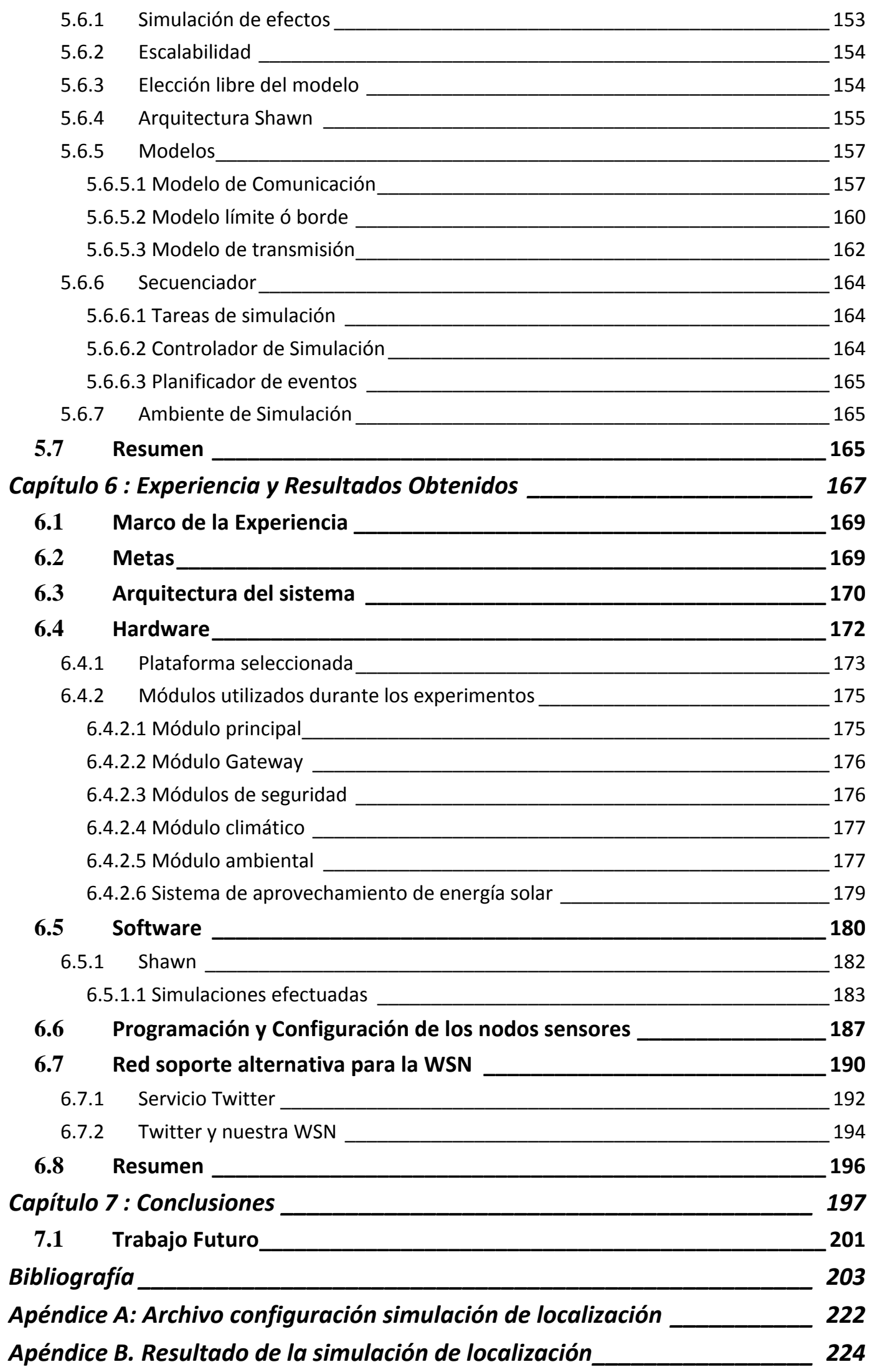


Apéndice C: Código programa instalado en nodos 228

Apéndice D: Script de Envío de datos a Twitter 235

Apéndice E: Trabajos relacionados al proyecto 236 


\section{Índice de Ilustraciones}

Ilustración 1.1 Plataforma de los prototipos Crosbow [11] 6

Ilustración 1.2 Espectro de costos de diferentes plataformas

Ilustración 2.1 Clasificación de redes inalámbricas por área de cobertura __ 28

Ilustración 2.2 Esquema de canalización de RF en IEEE 802.11_ 35

Ilustración 2.3. Evolución de la suite de protocolos 802.11 __ 36

Ilustración 2.4 Esquema típico de red inalámbrica ad hoc___ 39

Ilustración 2.5 Instalación típica de nodos sensores en una WSN __ 43

Ilustración 2.6 Alcance y tasa máxima de transferencia ___ 45

Ilustración 3.1 Proyección estimada de la penetración de las WSN en la sociedad___ 49

Ilustración 3.2 Clasificación de computadoras: [1] IBM S/360, [2] Apple II/IBM PCAT/XT, [3]

PCs/Amiga 486, [4] Teléfonos celulares a granel, [5] PCs Pentium __ 54

Ilustración 3.3 Nodos sensores en diques de contención___ 57

Ilustración 3.4 Monitoreo de petreles en la isla Great Duck___ 58

Ilustración 3.5. Arreglo de Sensores para monitoreo volcánico __ 59

Ilustración 3.6. Distribución de nodos para SHM en el puente Golden Gate [87] ___ 62

Ilustración 3.7. Auto organización de minas Self-Healing __ 63

Ilustración 3.8. Vista general de los componentes de un sensor ___ 77

Ilustración 3.9. Curva de descarga de Batería alcalina $A A_{2} 88$

Ilustración 3.10. Curva de descarga de batería de Litio __ 88

Ilustración 3.11 Módulo generación-almacenamiento de energía en nodos de WSN___ 92

Ilustración 3.12. Funcionamiento del controlador energético ___ 92

Ilustración 3.13. Estándares de comunicaciones inalámbricas y sus consumos___ 94

Ilustración 3.14 Consumo energético del circuito TI CC2430 en diferentes modos __ 97

Ilustración 3.15. AVIDirector___ 101

Ilustración 3.16. Dispositivo WMSN.P___ 101

Ilustración 3.17. Plataforma Jennic JN5139___ 102

Ilustración 3.18. Tecnología de MeshScape. a) Módulo MeshGate ___ 103

Ilustración 3.19. Nodo SensiNet EMS Smart___ 104

Ilustración 3.20. Nodo de la familia de sensores Sensinet RTD _ 104

Ilustración 3.21. Esquema de front-end de radiofrecuencia___ 108

Ilustración 4.1 Estructuras de las WSN___ 111

Ilustración 4.2 Arquitectura jerárquica en WSNs __ 112

Ilustración 4.3 Arquitectura distribuida en WSN __ 113

Ilustración 4.4 Diferencias operativas entre renvío y compilado de datos___ 114

Ilustración 4.5. Escenario de congestión y paliativos ___ 115

Ilustración 4.6 Enfoque holístico de la calidad de servicio ___ 121

Ilustración 4.7. Ejemplo de agregación adaptado de Holger and Willig [128]___ 129

Ilustración 4.8 WSN como base de datos __ 132

Ilustración 4.9 Pila de protocolos de Modelo Básico de Referencia OSI, TCP/IP y ZigBee ___ 134 
Ilustración 4.10 Pilas de arquitecturas existentes para WSN a) arbol de protocolos b) localización

c) tridimensional 135

Ilustración 4.11 Protocolos de cruce de capas para WSN 136

Ilustración 4.12 Dos formas de interfaz entre una aplicación y la pila de protocolos 137 Ilustración 5.1 Modelo de capas a ser simulado 142

Ilustración 5.2 Ámbito de aplicación de distintos simuladores WSN 148

Ilustración 5.3. Ciclo de desarrollo posibilitado por Shawn 155

Ilustración 5.4. Arquitectura de alto nivel de Shawn 156

Ilustración 5.5. Extracto de la Interfaz del Modelo de Comunicación de Shawn 157

Ilustración 5.6. Vista de los modelos de comunicación de Shawn 158

Ilustración 5.7. Modelo radial UDG 159

Ilustración 5.8. Modelo Radial RIM 160

Ilustración 5.9 Código de Interfaz aplicación del modelo de borde en Shawn 161

Ilustración 5.10 Modelos de borde de Shawn 162

Ilustración 6.1 Arquitectura de la implentación física 171

Ilustración 6.2 Nodo sensor iSense con porta baterías y cable USB 174

Ilustración 6.3. Módulo principal iSense 175

Ilustración 6.4. Módulo Gateway 176

Ilustración 6.5. Módulos de Seguridad utilizados 177

Ilustración 6.6. Módulo climático utilizado 178

Ilustración 6.7. Módulo ambiental utilizado 178

Ilustración 6.8 Módulo de administración de energía 179

Ilustración 6.9 Caja estanca con la batería y módulos montados 180

Ilustración 6.10 Entorno de Eclipse con Shawn y entorno de desarrollo de aplicaciones 181

Ilustración 6.11. Herramientas de evaluación de rendimiento de WSN según Imran 182

Ilustración 6.12 Archivo de configuración de Shawn para la simulación con 184

Ilustración 6.13. Pantalla de inicio de simulación 185

Ilustración 6.14. Simulación de localización en Shawn 186

Ilustración 6.15. Estructura de directorio instalada por el Firmware iSense 188

Ilustración 6.16 iSense generando aplicación en entorno Eclipse __ 189

Ilustración 6.17 Creación de ejecutable en entorno Eclipse 189

Ilustración 6.18. Ishell instalado aplicación en nodo 1c:22 191

Ilustración 6.19. Reportes de la WSN en el sitio web de Twitter 195 Ilustración 6.20. Datos de la WSN accedidos del sitio de Twitter 195 


\section{Índice de Tablas}

Tabla 3.1 Potencial energético para diferentes tecnologías de baterías 65

Tabla 3.2 Potencia generada por diferentes fuentes alternativas

Tabla 3.3 Banda de frecuencias disponibles de ISM para aplicaciones 95

Tabla 5.1 Simuladores típicos en WSN 147 


\section{Nomenclatura, Abreviaturas y Acrónimos}

\begin{tabular}{ll}
$\alpha$ & Coeficiente de pérdida de señal \\
$\mu$ & Densidad de Sensores \\
AMPS & Advanced Mobile Telephone System \\
AP & Access Point \\
ASIC & Application-Specific Integrated Circuits \\
CDMA & Code Division Multiplexing \\
DARPA & U.S. Defense Advanced Research Projects Agency \\
DECT & Digital Enhanced Cordless Telecommunications \\
EDGE & Enhanced Data Rate for GSM Evolution \\
EEPROM & Electrically-Erasable Programmable Read-Only Memory \\
ESB & Embedded Sensor Board \\
FPGA & Field-Programmable Gate Arrays \\
GPRS & General Packet Radio Service \\
GSM & Global System for Mobile communications \\
IEEE & Institute of Electrical and Electronics Engineers \\
IMT-2000 & International Mobile Telecommunications-2000 \\
ISM & Industrial, Scientific and Medical \\
IUSS & Integrated Undersea Surveillance System \\
LBS & Location Based Services \\
MAC & Medium Access Control \\
MANET & Mobile Ad Hoc Network \\
MBS & Mobile Broadband Systems \\
MEMS & Micro-Electro-Mechanical-System \\
NF & Relación de Ruido \\
NFC & Near Field Communication \\
PACS & Personal Access Communication System \\
PDA & Personal Digital Assistant \\
PPP & Point to Point Protocol \\
\hline FA
\end{tabular}




\begin{tabular}{|c|c|}
\hline PSTN & Public Switched Telephone Network \\
\hline PTT & Push-to-talk \\
\hline QoS & Quality of Service \\
\hline $\mathbf{R}$ & Confiabilidad \\
\hline RADIUS & Remote Authentication Dial-In User Server \\
\hline RAM & Random Access Memory \\
\hline RedVITEC & $\begin{array}{l}\text { Red de Vinculación Tecnológica de las Universidades } \\
\text { Argentinas }\end{array}$ \\
\hline RF & Radiofrecuencias \\
\hline RFID & Radio Frequency IDentification. \\
\hline ROM & Read only Memory \\
\hline SCADA: & Supervisory Control and Data Acquisition \\
\hline SMS & Short Message System \\
\hline SNR & Signal-Noise Relation \\
\hline sosus & Sound Surveillance System \\
\hline TACS & Total Access Communications System \\
\hline TDMA & Time Division Multiplexing \\
\hline UCB & Universidad de California en Berkeley \\
\hline UMTS & Universal Mobile Telecommunications Systems \\
\hline UWB & Ultra Wideband \\
\hline WEP & Wired Equivalent Privacy \\
\hline Wi-Fi & Wireless Fidelity \\
\hline WISEBED & Wireless Sensors Testbed \\
\hline WLAN & Wireless Local Area Network \\
\hline WiMAX & Worldwide Interoperability for Microwave Access \\
\hline WMAN & Wireless Metropolitan Area Network \\
\hline WMN & Wireless Mesh Networks \\
\hline WPA & Wi-Fi Protected Access \\
\hline WPAN & Wireless Personal Area Networks \\
\hline WSN & Wireless Sensors Networks \\
\hline WWAN & Wireless Wide Area Network \\
\hline
\end{tabular}


Capítulo 1 : Introducción 
E

continuo avance de la ciencia y la tecnología a lo largo del tiempo ha conllevado a un sinnúmero de innovaciones. Basta observar los avances que se han experimentado en la astronomía, basados en la mejora y evolución de las tecnologías de desarrollo de telescopios; como también los progresos a los largo de las últimas décadas en las ciencias médicas con todo lo referente al mundo microscópico. Cada una de estas innovaciones ha tenido que ver, indudablemente, con el desarrollo explosivo de las Tecnologías de la Información y Comunicaciones. Hoy es posible realizar medidas y cálculos a partir de las imágenes obtenidas por unidades satelitales, utilizando avanzados algoritmos computacionales para el cálculo.

Mark Weiser, conocido como el padre de la computación ubicua, a principios de los años 1990 ha apostado a la mencionada teoría, la que debería evolucionar hasta interactuar con los diferentes usuarios de las tecnologías [1], [2]. Estos principios enunciados originalmente por Weiser han sido validados por diferentes investigadores y estudiosos, convirtiéndose en un escenario cierto en el futuro cercano.

La computación ubicua tiene que ver, y está íntimamente relacionada, con la evolución de los dispositivos electrónicos. Esta revolución se materializa a través de la miniaturización de los dispositivos y la mayor sociabilización de los contenidos en red. Así hoy es posible realizar simulaciones numéricas para estudiar y analizar fenómenos utilizando métodos computacionales, los que se volverían prohibitivos si se pretendiera realizarlos por métodos empíricos.

La computación ubicua encuadra a un gran número de tecnologías y aplicaciones; desde dispositivos móviles utilizados diariamente, pasando por artefactos "inteligentes" para propósitos especiales (hornos, heladeras, etc.) y hasta a los juegos domiciliarios en red. 
La existencia de las numerosas y variadas simulaciones en los diversos campos de las ciencias se han producido como consecuencia del crecimiento exponencial de las propiedades y capacidades de los semiconductores que fueran desarrollados por la industria en los últimos años. De acuerdo a la ley de Moore, el número de transistores, y por ende la capacidad de almacenamiento y cálculo de un circuito; se duplica cada uno ó dos años [3]. Por ello, en la actualidad, es un desafío establecer y orientar los estudios hacia las regiones del conocimiento donde las TICs, convergen apuntalando a las ciencias.

Cuando el objetivo de una acción es obtener información válida de algún entorno con el cual se interactúa, la más de las veces es prácticamente imposible establecer vínculos fijos permanentes (cableados normales). Para estos escenarios se ha establecido una nueva tecnología, basada en un nuevo paradigma para los sistemas de cómputo, que han venido a denominarse redes de sensores inalámbricos (WSN).

Merece ser destacado aquí, por similitud operativa, la tecnología de identificación por radio frecuencia (RFID), que consiste en un sistema de almacenamiento y recuperación de datos remotos, utilizados por transponders ${ }^{1}$ RFID.

Con éste sistema, se transmite radialmente la identidad determinada de un objeto por medio de un código y/ó número de serie. Los elementos necesarios para ello son las etiquetas y los lectores. Los últimos, son los que detectan e identifican a determinado objeto físico, y que el mismo pueda ser controlado e inventariado en un sistema de cómputos al efecto.

Las etiquetas RFID se diferencian entre las pasivas y las activas. Las primeras carecen de una fuente de energía, pero son capaces de ser

\footnotetext{
${ }^{1}$ Set de emisión-transmisión que genera una señal de respuesta determinada bajo una interrogación electrónica apropiada.
} 
energizados desde el dispositivo que realiza la consulta. Los RFID activos son dispositivos provistos de alguna fuente de energía, guardando una relación con pequeños nodos integrados de WSN [4]. Recientemente han sido desarrollado dispositivos RFID clase 4 y 5 , los que son capaces de establecer entornos de seguimiento, ubicación, red y seguridad.

El deseo de mejorar el rendimiento de los servicios de las cadenas de provisión globalizadas ha impulsado el desarrollo de los sistemas RFID, pero su implementación ha sido problemática [4], en primer lugar por los costos de manufactura de las etiquetas y, fundamentalmente, por invasión de la privacidad de los usuarios adquirentes de productos etiquetados con RFID.

De estudios realizados en diferentes trabajos y publicaciones, se infiere el éxito de la computación ubicua; basado fundamentalmente en la adopción de las implementaciones de WSN y RFID. La ilustración 1.1 muestra la superposición existente entre las tecnologías RFID y las WSN.

Una red de sensores inalámbricos consiste en una serie de dispositivos distribuidos desordenadamente en un área geográfica dada. Cada nodo posee capacidad de comunicación inalámbrica, de procesamiento de la información obtenida y fundamentalmente, cada uno de los nodos es capaz de promover el establecimiento de una red de datos eficiente.

Las WSN han trascendido el ámbito académico-científico, habiéndose vuelto una realidad en cuanto a la utilidad práctica de la tecnología. El alcance y diversidad de las aplicaciones de las redes de sensores, los requerimientos, diseños y plataformas son virtualmente ilimitados [5]. 


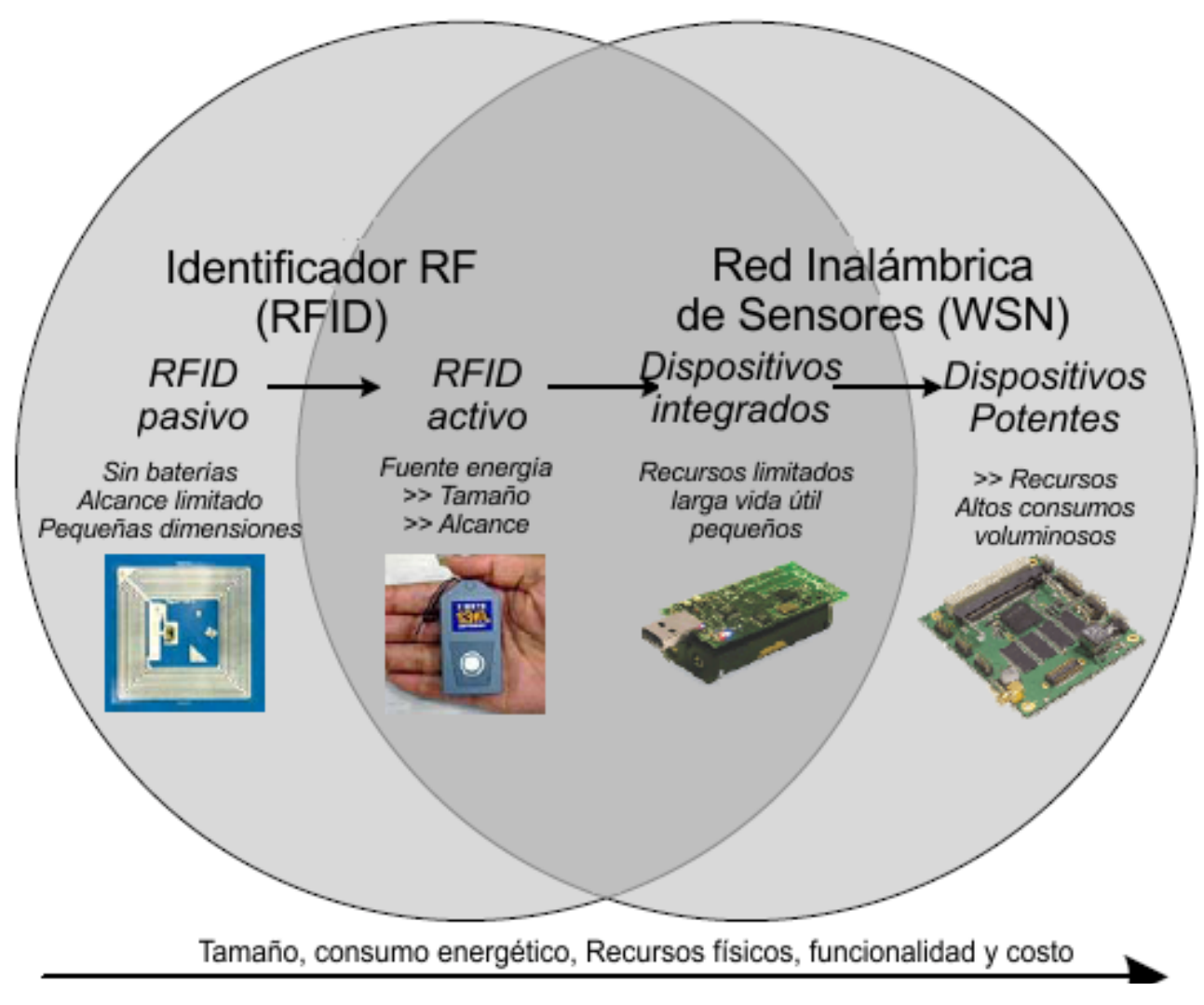

Ilustración 1.1. Intersección de las tecnologías de RFID y WSN

La génesis de las WSN se ha basado en el desarrollo de una serie de nodos por parte de la UCB en California. Los primeros equipos se denominaron nodos Mica [6]. Por razones operativas, y debido a partes del hardware poco confiables en estos equipos, los mismos evolucionaron a los nodos Mica2 [7], Mica2Dot y MicaZ [8]. El Mica2, como sucesor de los nodos Mica, proporciona una mejor interfaz de radio y diseño. El nodo Mica2Dot es bastante más pequeño que Mica2 y bastante más accesible en cuanto a precio. El nodo MicaZ se caracteriza porque reemplaza el sistema de radio propietario por otro compatible del tipo IEEE 802.15.4. Como nodo sucesor de Mica2 aparece el Telos [9], [10] en 2004. Este nodo se orienta fundamentalmente a atacar las debilidades de la 
tecnología: el consumo de energía, cortos tiempos de wake-up y la utilización de radio IEEE 802.15.4.

\begin{tabular}{|c|c|c|c|c|c|}
\hline \multicolumn{2}{|c|}{ Mote type } & IMote2 & MicaZ & Mica2 & Mica2Dot \\
\hline \multicolumn{6}{|c|}{ Example picture } \\
\hline \multirow{4}{*}{ MCU } & Chip & Marvell PXA271 & MPR2400CA & \multicolumn{2}{|c|}{ ATmega128L } \\
\hline & Type & 13-416 MHz, & & \multicolumn{2}{|c|}{$8 \mathrm{MHz}, 8$ bit } \\
\hline & Program memory & 32 МB & \multicolumn{3}{|c|}{$128 \mathrm{~KB}$} \\
\hline & RAM (KB) & 258 & - & & \\
\hline \multirow{3}{*}{$\begin{array}{l}\text { External } \\
\text { nonvolatile } \\
\text { storage }\end{array}$} & Chip & - & - & \multicolumn{2}{|c|}{ AT45DB014B } \\
\hline & Connection type & $12 \mathrm{C}$ & I2C, SPI & \multicolumn{2}{|c|}{ SPI } \\
\hline & Size (KB) & - & - & \multicolumn{2}{|c|}{512} \\
\hline \multirow{2}{*}{$\begin{array}{l}\text { Default power } \\
\text { source }\end{array}$} & Type & $3 \times A A A$ & \multicolumn{2}{|c|}{$\begin{array}{l}\text { 2xAA } \\
2 \times A A \\
2 \times A A\end{array}$} & Coin cell \\
\hline & $\begin{array}{l}\text { Typical capacity } \\
\text { (mAh) }\end{array}$ & 500 & \multicolumn{2}{|c|}{2850} & 1000 \\
\hline \multirow{3}{*}{ RF } & Chip & CC2420 & - & & \\
\hline & Radio frequency & \multicolumn{2}{|c|}{$2.4-2.4835 \mathrm{GHz}$} & \multicolumn{2}{|c|}{$868 / 916 \mathrm{MHz}, 433$, or $315 \mathrm{MHz}$} \\
\hline & Raw speed (kbps) & \multicolumn{2}{|c|}{250} & \multicolumn{2}{|c|}{38.4} \\
\hline
\end{tabular}

Ilustración 1.1 Plataforma de los prototipos Crosbow [11]

Montados en la ola paradigmática, se desarrollaron en Europa varias plataformas. Se destacan tecnologías como el BTnode [12], [13] que proporciona conexión a notebooks, PDAs y teléfonos celulares por Bluetooth. La Freie Universität Berlín, ha desarrollado y establecido el ESB (Embedded Sensor Board) [14], que luego evolucionara a la marca comercial Scatternode [15]. Demostrando el interés y desarrollo del campo, la plataforma iSense [16] se desarrolla en Alemania, en un todo de acuerdo a los estándares comunes imperantes. 


\subsection{Porque Una WSN?}

Los objetivos básicos del establecimiento de una red de sensores son función directa de la aplicación necesaria de la red a establecer. Normalmente la tareas comunes a la mayoría de las redes de éste tipo son: medición, monitoreo y control. Las tareas mencionadas son orientadas a realizar alguna de las siguientes tareas:

1. Determinación del valor de algún parámetro en un lugar geográfico determinado. Se puede pretender saber la temperatura, presión atmosférica, la intensidad de iluminación, y la humedad relativa en un número definido de ubicaciones.

2. Detección la ocurrencia de eventos de interés. Discriminar y clasificar a los parámetros para una evaluación/estimación de los eventos indicativos de aquello que se considere de interés.

3. Clasificación un objeto detectado: Clasificar el objeto detectado en el paso anterior.

4. Monitoreo de un objeto: Seguimiento de un elemento determinado que se mueve por la zona geográfica cubierta mediante la red de sensores

De las cuatro tareas mencionadas, la característica común en todas ellas es que la medición debe ser transmitida a los usuarios finales de una manera segura, salvaguardando los datos obtenidos. En ciertas ocasiones es imperativa la transmisión inmediata de los datos obtenidos, para así poder implementar medidas correctivas y/ó de seguridad. Un incendio, robo ó ataque a la propiedad son eventos de ésta naturaleza.

Las redes ad hoc son sistemas sumamente complejos. En ellos conviven y participan muchos conceptos, protocolos, tecnologías, algoritmos, y elementos que deben ineludiblemente trabajar conjuntamente. La 
aplicación de las redes móviles ad hoc (MANET) y las WSN es sumamente diversa, yendo desde pequeñas redes estáticas limitadas en su existencia por la necesidad de disponibilidad de energía, a redes a gran escala con gran dinámica y mucha movilidad. Las WSN son unas de las herramientas preferidas en SCADA con el objeto de monitorear 0 controlar procesos químicos u operaciones de fenómenos de transporte. Son frecuentemente utilizadas en el ámbito de la producción y suministro de agua potable, como también en entornos de generación energética [17].

Existen muchas razones que deben ser consideradas en el momento de formular un proyecto de redes de datos, pero dos de ellas posicionan a las WSNs mejor que a otras tecnologías: la económica y la de aplicación.

Según la literatura, el beneficio de las WSN frente a otras tecnologías convencionales, deviene del costo necesario para la realización del cableado entre sensores. El costo de instalación de un simple nodo alcanza los US\$ 200 en un edificio de oficinas [18], o puede alcanzar valores como US\$ 150 por cada metro de instalación en entornos peligrosos, tales como son consideradas las industrias químicas o plantas generadoras de energía. La adopción de sistemas inalámbricos de sensores puede proveer una reducción de costos considerables, hasta un $80 \%$ del costo total, en materiales y mano de obra.

\begin{tabular}{c|c|c|c|c|c}
\hline RFID & $\begin{array}{c}\text { Wireless Sensor } \\
\text { Nodes }\end{array}$ & $\begin{array}{c}\text { Gateways/ } \\
\text { Aggregators }\end{array}$ & Handheld & $\begin{array}{c}\text { Mobile/ } \\
\text { Desktop }\end{array}$ & Server \\
\hline US\$ 0,1 & US\$1 1 & US $\$ 10$ & US $\$ 100$ & US $\$ 1.000$ & US $\$ 10.000$
\end{tabular}

Ilustración 1.2 Espectro de costos de diferentes plataformas de cómputo según Culler 
En la ilustración 1.2 se muestra el espectro de costos de las distintas plataformas de cómputos existentes en el mercado [19].

\subsection{Justificación de la investigación}

El objetivo práctico de este trabajo es el desarrollo, prueba, configuración y puesta en marcha de una infraestructura de red de sensores inalámbricos. Esta infraestructura a nivel piloto, debería ser utilizada en primer lugar para fines de investigación, apuntando a una visión interdisciplinaria que abarca los aspectos del hardware, software, algoritmos y datos.

Se pretende demostrar que la heterogeneidad de dispositivos de pequeñas dimensiones y instalaciones piloto (testbeds) existentes, pueden unirse para formar estructuras a gran escala bien organizadas; diferentes de las grandes redes actuales, dado que permitirán una investigación cualitativa y cuantitativa a una escala mucho mayor, afrontando los cambios dinámicos de escenarios, infraestructura y composición de nodos.

Las redes de sensores inalámbricos son una de las tecnologías fundamentales en la Internet del futuro, y representa la expansión de Internet al mundo real. Con esto se permite acceder a indicadores desde cualquier punto del orbe en tiempo real.

Un aspecto sobresaliente de la "Internet del Futuro" es la utilización de tecnología sumamente compleja, entre las que se puede establecer la utilización de una red de sensores de una manera totalmente transparente al usuario. Con ellos tiende a favorecer la integración de las redes fijas a las nuevas tecnologías de "conectividad móvil".

El proyecto ha sido enmarcado en el nacimiento en Europa de un consorcio de instituciones bajo el nombre de WISEBED [20]. Este 
consorcio está formado por la Universidad de Lübeck, Universidad de Berlín, Universidad de Braunschweig de Alemania, el Research Academic Computer Technology Institute de Grecia, la Universitat Politècnica de Catalunya de España, Universidad de Berna y la Universidad de Ginebra de Suiza, Universidad de Delft de Holanda, y la Universidad de Lancaster en el Reino Unido.

WISEBED forma parte de la "Estrategia Europea para Internet del Futuro", la que se está discutiendo a nivel nacional en Alemania y respaldada en su accionar, por la Sociedad Alemana de Informática.

El proyecto aquí desarrollado ha sido imaginado como un apéndice de WISEBED, dado que el fin último es instalar, configurar y activar una red de sensores inalámbricos en la Republica Argentina, la que una vez operativa se integraría al consorcio mencionado como la primera no europea.

Actualmente no existe en nuestro país una red de sensores realmente establecida que pueda servir como estación piloto para alguna inquietud de investigación y/ó desarrollo con socios nacionales y/ó extranjeros. Como consecuencia de este trabajo, se establecerá una red básica de sensores, la cual servirá para el desarrollo e investigación de distintos grupos dedicados al tema en nuestro país.

Se ha considerado a este trabajo como un aporte al establecimiento de una red global de sensores donde los humanos (agentes inteligentes), y computadoras potentes interactúan con redes piloto de sensores inalámbricos, distinguiendo en la red global 3 subdominios:

- Una red superior existente, donde los distintos nodos ejecutan las aplicaciones en potentes computadoras que pueden interconectarse vía Internet u otras redes globales. 
- Los dispositivos sensores que forman una red piloto y se comunican entre sí por medio inalámbrico.

- Un portal de servidores, formado por nodos que controlan las redes piloto, y permiten la interacción entre los nodos de la red superior y los dispositivos de la WSN.

Los temas a desarrollar en esta tesis son:

- Modelado y simulación: La simulación es utilizada para evaluar sistemas desarrollados y a implementar en redes reales. La utilidad de los datos obtenidos depende puramente del realismo y la precisión de los modelos a implementar. Fundamentalmente se avanzan con simulaciones que simplifican la instalación de las WSNs.

- Entorno de desarrollo de aplicaciones: Necesario para la corrida de las simulaciones como también desarrollo de las aplicaciones a ser instaladas en los nodos físicos existentes.

- Alternativa de soporte de las WSN: la preexistencia de situaciones donde la prestación de servicios de transmisión de datos es sumamente ineficiente y provisto por una empresa de manera monopólica. Ciertas veces el servicio es inexistente en la locación del proyecto. Por ello se avanzó en la búsqueda de un soporte a la red de WSN no basada en la Red Digital Soporte de la prestadora en la región de desarrollo del trabajo.

\subsection{Contribuciones del presente trabajo}

Las redes de sensores inalámbricos son hoy una realidad que permite una gran variedad de aplicaciones en el área de las detección/medición de eventos en vastas superficies de terreno de manera inalámbrica, 
remota y distribuida. Este tipo de nodos integrantes de la red son de un tipo muy especial, dado que posen una capacidad restringida en cuanto a vida útil, cálculo y memoria. Estas restricciones generan campos definidos hacia donde la investigación y desarrollo debe avanzar inexorablemente, desarrollando modelos y aplicaciones para estos pequeños y avanzados equipos de comunicación y cálculo.

Se pueden enumerar las siguientes contribuciones:

- Establecimiento de una red piloto: Se establece una red piloto con disponibilidad de nodos, posibilidad de soporte a la investigación y desarrollo de actividades orientadas a las WSN. Esta red servirá de trampolín para futuras investigaciones y desarrollos sobre el tema, dado que no existe alguna similar en nuestro país.

- Transferencia tecnológica y know-how: El proceso de apropiación de la tecnología, por medio de la asociación con instituciones europeas ha permitido disminuir el tiempo de transferencia, con lo que se abre una puerta a las investigaciones sobre el tema en la red piloto implementada.

- Adopción de Shawn: Habiendo evaluado varios entornos de simulación, se ha adoptado uno que podrá ser profundizado en trabajos posteriores. A la fecha existe un tesis de graduación de la Licenciatura en Sistemas de Información en la Universidad Misiones sobre el tema "Sistemas Modernos de Comunicación", utilizando como base de desarrollo a Shawn.

- Formación de un grupo de trabajo sobre WSN: por la existencia del presente trabajo se ha aglutinado un grupo de profesionales y estudiantes que han adherido a la línea de investigación, desarrollando diferentes tareas al respecto. 
- Soporte de las WSN por una red social: a la pobre oferta de la proveedora de conectividad en la zona del proyecto y a los efectos de resguardar el éxito de los emprendimientos, se ha desarrollado una estructura donde Twitter es utilizada como soporte de la WSN implementada.

\subsection{Resultados y Conclusiones}

- Se ha realizado un estudio del estado del arte y evolución de las WSN hasta la fecha.

- La Red de sensores inalámbricos instalada en forma piloto se comportó en forma robusta.

- Se alcanzaron las metas propuestas como orientaciones al trabajo a realizar en el proyecto.

- Ante inconvenientes de índole práctico que se presentaron a lo largo de las tareas planificadas, se encontraron las soluciones ingenieriles correspondientes.

- La administración remota de la red instalada, se devela como un punto crítico en cuanto a la utilización y al futuro de la tecnología.

- Se han desarrollado e implementado aplicaciones distribuidas y totalmente integradas.

- Se ha confirmado una vez más la imprevisibilidad del medio ambiente en las experiencias llevadas a cabo.

- Se han integrado el simulador seleccionado, el firmware del hardware implementado y un entorno de aplicación y desarrollo; en un único entorno amigable. 
- Se ha experimentado con protocolos de alto nivel de transmisión inalámbrica basados en 802.15 .4

- La red piloto que se pretendía como uno de los elementos del proyecto, se encuentra actualmente operativa.

- Se compararon resultados de simulaciones con experiencias realizadas en el mundo real.

- Se han investigado soportes alternativos de conectividad a redes externas, para así permitir el acceso de los usuarios a los resultados de la experiencia. En este trabajo se integró a la red social Twitter como soporte de la WSN.

- Se han identificado y clasificado cada uno de los roles cumplidos por los nodos en la WSN instalada.

- Hemos implementado entornos de simulación con resultados replicables en campo (cálculo y visualización).

- Se ha establecido la muy buena integración y escalabilidad de las redes experimentales establecidas durante el proyecto.

\subsection{Publicaciones y participación en Eventos}

Como resultado del presente trabajo se han presentado a conferencias, reuniones y otros eventos en el país y el exterior, cuyas copias se encuentran en el Anexo E:

> Eduardo Sosa, Stefan Fischer, Javier Díaz: Towards a Global Sensor Network Interconnection: A German-Argentinean Field Trial, 2nd EELA-2 Conference, Choroni, Venezuela, Nov. 25-27, 2009. ISBN: 978-84-7834-627-1. 
Eduardo O. Sosa; Stefan Fischer , Francisco J. Díaz, et ál., Twitter, Soporte de una Red de Sensores Inalámbricos, XII Reunión de Otoño de Potencia, Electrónica y Computación, ROPEC'2010 INTERNACIONAL, Manzanillo, Colima, México. (enviada: http://www.ieee-sco.org/ropec/resultados_ropec_2010.html)

$>$ Hacia una Red Global de Sensores Interconectados. Un ensayo experimental Argentino-Alemán. Eduardo O. Sosa, Francisco J. Díaz, Luis A. Marrone. II Jornadas RedVITEC. La Vinculación Tecnológica en el contexto de las Políticas de Estado y la Sociedad. Entre Ríos, Paraná, 19 y 20 de Noviembre de 2008. ISBN: 978-950-698-234-8

Universidad Nacional de Misiones; Facultad de Ciencias Exactas, Químicas y Naturales. Secretaría de Investigación y Posgrado. Proyecto de Investigación: "Hacia la programación de Sensores Inalámbricos en la forma web 2.0".

$>$ Proyecto AL807 "Hacia una Red Global de Sensores Interconectados. Un ensayo experimental Argentino-Alemán", presentado en el marco del Programa de Cooperación CientíficoTecnológico entre el Ministerio de Ciencia, Tecnología e Innovación Productiva de la República Argentina (MINCYT) y el Bundesministerium für Bildung und Forschung (BMBF) de Alemania. Integrante. Directores: Francisco Javier Díaz y Stefan Fischer.

\subsection{Estructura del trabajo}

En el capítulo 2 se realiza un estudio de la evolución de las redes inalámbricas a través del tiempo. Para ello se contempló lo atinente a los tipos de servicios inalámbricos que se prestan en la actualidad, 
analizando las redes existentes y clasificándolas por la estructura subyacente de la red y el área de cobertura de la misma. Se realiza un análisis de la evolución de las redes móviles celulares hasta la tecnología utilizada actualmente, arribando luego a la suite 802 de la IEEE. Se estudia la evolución de las redes ad hoc, en especial se realza la división existente entre redes ad hoc móviles y las redes de sensores inalámbricos, para finalmente llegar a redes de malla.

En el capítulo 3 se comienza a tratar lo concerniente a los sensores inalámbricos y la escala. En todo momento se considera y se refuerza el concepto de la existencia y aplicación de un nuevo paradigma el campo de la computación. Se presentan los diferentes escenarios de aplicación de la tecnología tratada en la tesis, introduciendo los factores de diseño de las redes de sensores y las métricas de evaluación, tanto de los sensores en sí mismo como de las redes en particular. Se presentan y analizan las distintas alternativas referentes a las prestaciones de los diferentes componentes de hardware de los nodos.

En el capítulo 4 se presenta la arquitectura de red de las WSN, clasificando las funciones posibles de los diferentes nodos en la red. Se considera la movilidad de los nodos, tanto de los dispositivos comunes como los que cumplen tareas de límite de red. Se consideran los objetivos de optimización, como los principios de diseño. Al final del capítulo se analiza cierto comportamiento de las redes de sensores que contradicen la norma en cuanto a las pilas de protocolos e interfaces de servicio.

En el capítulo 5 se comenta sobre los diferentes simuladores existentes y una clasificación de acuerdo a sus rendimientos, referente al entorno de utilización y número de nodos de las simulaciones. Una vez que se seleccionó el simulador a utilizarse en el trabajo se ahondó en las características del mismo. 
En el capítulo 6 se enumera lo atinente a equipamiento y programas utilizados, indicando las diferentes plataformas y productos que se acoplaron para fundamentar el desarrollo de las aplicaciones instaladas en los nodos, como también los resultados obtenidos tanto de las simulaciones efectuadas, como de las tareas de proveer a la red piloto de sensores de un soporte de publicación de sus tareas, que sea una alternativa válida a los proveedores monopólicos de conectividad.

Finalmente en el capítulo 7 se discuten los resultados y proponen tareas a futuro sobre la tecnología analizada en la tesis. 
Capítulo 2: Evolución de las

Redes Inalámbricas 


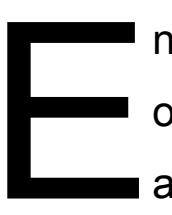

$\mathrm{n}$ el mundo actual donde las tecnologías inalámbricas van ocupando un lugar cada vez más importante, no cabe imaginarse al hombre como una entidad prescindente de la misma. Todo servicio y aplicación se encuentra como mínimo en sus dos opciones, cableado e inalámbrico. En cada uno de los diferentes estratos de nuestra sociedad se evidencia la importancia de los servicios móviles en la interacción día a día con la realidad.

Hace poco tiempo, no más que una década, los servicios a los que se podía acceder de manera inalámbrica no iban más allá que el servicio básico de comunicación por voz. Hoy cada uno de los estamentos de la sociedad en su conjunto es impactado de diferente manera por las tecnologías inalámbricas. Podemos dar fe de grandes aportes y/ó dependencia de los servicios móviles en la educación, la salud, entretenimientos; y en manera muy especial todo aquello referente al comercio. La evolución de las tecnologías de transmisión inalámbricas ha aportado de manera sustancial en el mejoramiento de la calidad de vida de usuarios en diversos puntos del orbe.

El estudio, proyección e instalación de tecnologías inalámbricas se ha convertido en la parte más importante y desafiante de la telemática de nuestros dias. Las aplicaciones inalámbricas actuales: telefonía celular, redes de área local inalámbrica (WLANs), los sistemas satelitales de posicionamiento global (GPS), sistemas de distribución multipunto local (LMDS), sistemas de identificación por radio frecuencia (RFID), redes de sensores inalámbricos (WSN), redes móviles ad hoc (MANET), etc.; son parte importante del mercado global, y experimentan un crecimiento y evolución continua [21]. Esta evolución ha comenzado en 1973 el día que Martin Cooper realizó la primera llamada por telefonía celular a Joel Engel de la Bell Labs. Coincidentemente IBM publicaba los resultados obtenidos en su fábrica en Suiza de experimentos realizados sobre la manera de interconectar varios equipos por medio de trasmisiones en el espectro 
infrarrojo. Ambos momentos, son el punto histórico de quiebre para el desarrollo y avance de todo lo atinente a las tecnologías inalámbricas sin solución de continuidad.

No es extraño entonces, que todos y cada uno de los individuos de la sociedad actual seamos dependientes de la tecnología, de modo tal que se conforma en una ayuda en las necesidades cotidianas de la vida. En un día típico, más de dos mil millones de personas en todo el mundo confían en las ofertas de servicios móviles para coordinar, realizar y avanzar en su rutina diaria.

\subsection{Servicios móviles actuales}

De aquello que encontramos como oferta en el éter, consideramos a los siguientes como los servicios móviles más representativos: la comunicación, las empresas, los entretenimientos, localización y ubicación, salud, educación a distancia, comercio móvil y otros servicios móviles emergentes

La comunicación móvil has sido causante principal del rápido cambio de los escenarios en cuanto al por qué, cuándo, con qué frecuencia y en qué grado nos comunicamos. Estas comunicaciones pueden ser ubicuas, personal o interactivas [22]; convirtiéndose en uno de los servicio más comunes, fáciles y entretenidos.

Quienes han sido los precursores de la utilización de la tecnología inalámbrica para sus tareas habituales han sido las diferentes empresas móviles. Por sus particularidades, éstas han estado siempre a la vanguardia de la adopción temprana de servicios de tecnología inalámbrica. Con ello han logrado una ventaja competitiva respecto a la competencia. Esta ventaja se vislumbra en un incremento de la eficiencia [23], debido a la integración a los procesos de negocio desde localizaciones fijas, con la fuerza laboral móvil. 
El creciente número de aficionados a los diferentes entretenimientos actuales, ha visto con sumo agrado no tener que estar de cuerpo presente en sus hogares para poder satisfacer su pasión por el entretenimiento. Con el arribo de las tecnologías inalámbricas, han logrado la flexibilidad y libertad no solo de participar, sino también elaborar con sus preferencias la programación particular de su entretenimiento [24], [25]. Hoy, y cada vez más frecuentemente, la televisión móvil, vídeos y películas, música, juegos, o la generación de contenido en las diferentes redes sociales son elementos al alcance de cualquier usuario.

Una de las maneras de personalizar los servicios móviles, es basar los mismos de acuerdo al posicionamiento de los distintos dispositivos móviles por medio de LBS (Location Based Services). El principal de los sistemas utilizados es el de posicionamiento global (GPS), pudiendo utilizarse asimismo el método de triangulación de las señales desde distintos nodos proveedores del servicio inalámbrico. Para algunos, LBS se corresponde con el seguimiento eficiente de los bienes y servicios. Para otros, LBS aumenta el nivel de confort, permitiendo, por ejemplo, la ubicación de un niño o un anciano. En tiendas y restaurantes, LBS proporciona instrucciones precisas para un cliente cualquiera [26].

Los servicios móviles de salud son conocidos como telemedicina o telesalud. Están diseñados para permitir una mejor calidad de vida a los usuarios por medio de tratamiento ambulatorio y contralor de la evolución de pacientes. Así, es posible la captura de los datos médicos intrínsecos a la patología de cada uno de los pacientes de manera ambulatoria, lo que agiliza y permite el diagnóstico y tratamiento oportuno [27]. Los servicios móviles de asistencia sanitaria proporcionan libertad, movilidad y un mayor sentido de bienestar a los pacientes externos.

El comercio móvil es el campo que mayor impulso y desarrollo ha obtenido con la implementación de tecnologías inalámbricas. El Ilamado 
muchas veces comercio electrónico inalámbrico, consiste en la propaganda y compra/venta de bienes y servicios a través de redes de comunicación de datos inalámbricas, utilizando como interfaces a dispositivos móviles [28]. "Tiempo es dinero" sigue representando a los intereses de la caótica y veloz economía de hoy; con los ya establecidos omnipresentes servicios móviles de comercio como e-banking, e-pago, el dinero electrónico, etc. [29].

La educación no ha sido la excepción en involucrarse en la utilización de la tecnología inalámbrica. En los últimos años se ha sociabilizado a través de la red los comúnmente denominados servicios móviles de educación a distancia. Con éstos se permiten nuevas formas de relacionamiento docente/alumno; generándose nuevas experiencias de enseñanza y aprendizaje [30]. Se ha maximizado la accesibilidad a estos servicios de manera de poder recibir instrucción en un aula en vivo (o en caché); o a la formación profesional en un entorno móvil o de enseñanza a distancia.

Por último cabe mencionar aquí a los servicios móviles emergentes. Algunos de estos proporcionan posibilidades de capacitación en cuestiones sociales y económicas a muchos ciudadanos que nunca pensaron podrían ser destinatarios de dichos servicios [31]. La gente, en los mercados emergentes, está adoptando los servicios móviles que, tradicionalmente, no estaban disponibles para ellos, tales como banca móvil, el aprendizaje a distancia y la telemedicina.

Los servicios que acabamos de revisar son solo una muestra de los muchos que se ofrecen actualmente. En el futuro, la forma en que utilizamos los servicios móviles seguirá creciendo y evolucionando, debido a que nuestra imaginación no posee límites en cuanto a mejorar la calidad de vida propia y de nuestros semejantes. 


\subsection{Desarrollo de las comunicaciones inalámbricas}

El sector de comunicaciones inalámbricas ha experimentado un crecimiento exponencial en la década pasada. A lo largo del tiempo las instalaciones de las topologías de red en diferentes estamentos fueron alcanzando un delicado equilibrio entre necesidades de conectividad en edificios, aplicaciones a instalar y ancho de banda requerido. La evolución desde las conexiones cableadas conmutadas Ethernet, a conexiones compartidas inalámbricas, ha roto el equilibrado balance anterior, determinando que el ancho de banda sea el factor potencialmente limitante en las instalaciones.

Podemos considerar varios parámetros cuando pretendemos realizar una evaluación del desarrollo del sector de las tecnologías inalámbricas. Es conveniente analizar por lo tanto las variaciones en las infraestructuras de red, el crecimiento de usuarios de comunicación móvil de voz, la disponibilidad de aplicaciones inalámbricas en mayor cantidad y la aparición de los nuevos dispositivos inalámbricos. Estos dispositivos, que han evolucionado conjuntamente en cuanto a potencia y capacidad de cálculo, están conformados por ordenadores portátiles o de mano, PDAs y teléfonos móviles. Podemos afirmar que los dispositivos móviles actuales poseen características de mejor portabilidad, más económicas, son confortables y poseen potencia considerable.

En la actualidad los usuarios son capaces de revisar sus cuentas de email o navegar en el WWW directamente desde sus teléfonos celulares, y dado la ubicuidad de las señales, no existe límite para el establecimiento de conectividad.

Es normal en nuestros días el uso de herramientas posicionamiento global instaladas en automóviles, de manera de recorrer las rutas y caminos, evitando la utilización de los históricos mapas de ruta. Esta 
herramienta permite localizar y establecer derroteros hacia lugares de interés turísticos.

Todos los factores que hemos mencionado sucintamente hasta aquí, son catalizadores del crecimiento explosivo y desmesurado del mercado de la comunicación celular. Estudios independientes han demostrado que los usuarios de celulares se han ido duplicando progresivamente cada año y medio. Conjuntamente con las redes celulares, se ha incrementado notablemente el uso de los accesos inalámbricos a redes estáticas por medio de Puntos de Acceso (AP).

\subsection{Clasificación de Redes Inalámbricas}

A las redes inalámbricas las catalogamos teniendo en cuenta:

- la arquitectura de la red

- $\quad$ el área de cobertura de la red

\subsubsection{Redes basadas en arquitectura}

La arquitectura de una red define los protocolos y componentes que son necesarios de manera de satisfacer los requerimientos de la aplicación. El más popular de los estándares de ilustración de la arquitectura de red es el modelo de referencia de las siete capas de la OSI (Open System Interconnection) que fuera desarrollado por la International Standards Organization (ISO).

La arquitectura de red es la forma más económica y efectiva de desarrollo e implementación de conectividad entre un determinado conjunto de equipos. Tiene que ver principalmente con la forma en la cual se conectan los protocolos y elementos del software. Esto es algo que redunda en beneficio de los usuarios de la red, como también de los proveedores de hardware y software. 
Las redes inalámbricas, por construcción, permiten que las aplicaciones implementadas también sean móviles y portables. Las claves para la comunicación inalámbrica son las interfaces de red (NICs) y las estaciones base; quienes utilizan al aire como medio de transporte.

\subsubsection{Redes basadas en infraestructura}

Este tipo de redes posee una estructura fija, lo que normalmente está conformado por una serie de nodos y puertas de enlace los que se distribuyen en algún área física. Las estaciones base proveen el acceso al backbone de red cableado desde los nodos móviles. Las funciones de control de red son realizadas por las estaciones base, y generalmente existe un vínculo entre ellas de manera de simplificar lo referente a control.

Pertenecen a éste tipo de redes la telefonía celular, las redes inalámbricas locales (WLANs) y los sistemas de paginado. Estos sistemas están construidos de manera que cada una de las terminales móviles transmite directamente a una estación base, no utilizando para ello el ruteo multisalto entre nodos intermedios. Con estas rutas "single-hop" obtienen menores retardos, pérdidas de paquetes, mayor tasa de transferencia y flexibilidad [32].

Por todas estas razones se explica el mejor rendimiento de las redes inalámbricas basadas en infraestructura, comparadas con la que no son basadas en ella. Las redes celulares son un tipo de estas redes que hacen una utilización eficiente del espectro radial, reutilizándolo nuevamente en diferentes ubicaciones separadas espacialmente. Están constituidas normalmente por la red telefónica básica (PSTN), los centros móviles de conmutación, las estaciones base y los host móviles. 


\subsubsection{Redes sin infraestructura}

Este tipo de redes inalámbricas son muy importantes y las de mejor desempeño en situaciones de demanda temporal y/ó localizada del servicio de telecomunicaciones. Se caracterizan por formarse dinámicamente y de una forma cooperante entre diversos equipos inalámbricos, sin necesidad de una organización previa de los roles específicos a cumplir por cada nodo en la red. Cada equipo es capaz de reenviar un paquete a un determinado destino, si dicha tarea le fuera solicitada. Además, cada nodo es suficientemente independiente como para tomar una decisión de transporte basada en la situación existente en la red.

En la última década se ha dedicado un importante esfuerzo en lo que se refiera a investigación [33] de uno de estos tipos de redes, las redes móviles ad hoc (MANET). Aparte de su capacidad de auto configurarse y enlazarse, los dispositivos en las MANETs realizan tareas de ruteo, auxiliándose unos a otros para así transferir la información de una manera eficiente. La implementación de este tipo de redes es fundamental en casos de desastres naturales, operaciones de rescate, o situaciones específicas donde la infraestructura de red ha sido destruida, o frente a la inexistencia de la misma. Basta recordar algunas de las situaciones de desastre ocurridas últimamente, donde ha quedado demostrado que las redes tradicionales de comunicaciones han ayudado muy poco. En los Estados Unidos de América, basta recordar las consecuencias devastadoras del el huracán Katrina ${ }^{1}$, como el desastre ecológico en el Golfo de México ${ }^{2}$ por la explosión de uno de los pozos de extracción de gas de la firma BP.

\footnotetext{
${ }^{1}$ fue un gran ciclón tropical que azotó el sur y el centro de los Estados Unidos en agosto de 2005.

${ }^{2}$ plataforma petrolera "Deep Water Horizon", que se encuentra a unos 70 km de la costa, frente a Nueva Orleans, EUA.
} 
Si bien se ha avanzado mucho en las investigaciones sobre MANET en los últimos años, la adopción de la tecnología dista mucho de ser la preferida del mercado inalámbrico, contrariamente a lo indicado por el potencial de la misma.

Si bien es atrayente y cautivadora la flexibilidad de la comunicación anytime-anywhere ofrecida por las MANET, estas redes no fueron diseñadas para proveer un servicio confiable, deseado para el caso de aplicaciones de red sensibles a retardos, como las acciones de rescate ó en los campos de batalla. Normalmente existe en estas redes un número de nodos que se desconectan aleatoriamente debido a movilidad, daños ó desperfectos. Estos períodos de inactividad pueden ser bastante prolongados, y durante la ocurrencia de estos percances puede existir pérdida de información sensible y degradación de la performance de la red. Lo anterior ocurre debido a inconvenientes tanto en las tareas de ruteo, como en los protocolos en la capa de transporte. La característica más sobresaliente de estas redes es el comportamiento dinámico y cooperativo de cada uno de los nodos para el establecimiento espontáneo de la red. Las redes de sensores inalámbricos (WSN), también forman parte de este tipo de redes.

\subsubsection{Redes basadas en el Área de Cobertura}

El criterio usual para caracterizar a las redes es hacerlo de acuerdo al alcance o a la escala de cobertura. Razones históricas han llevado a considerar cada uno de estos tipos de redes como para algún tipo de área. Ejemplos típicos son mostrados en la ilustración 2.1.

\subsubsection{Redes Inalámbricas de Área Amplia (WWAN)}

Para el establecimiento de conexiones inalámbricas a redes remotas, estas son redes dependientes de otras redes basadas en infraestructura. 
Las áreas cubiertas son normalmente bastante extensas; abarcando en general ciudades y ciertas veces países. Utilizan en la mayoría de las instalaciones sistemas de múltiples antenas, ó saltos satelitales, que generalmente son administrados por los proveedores del servicio.

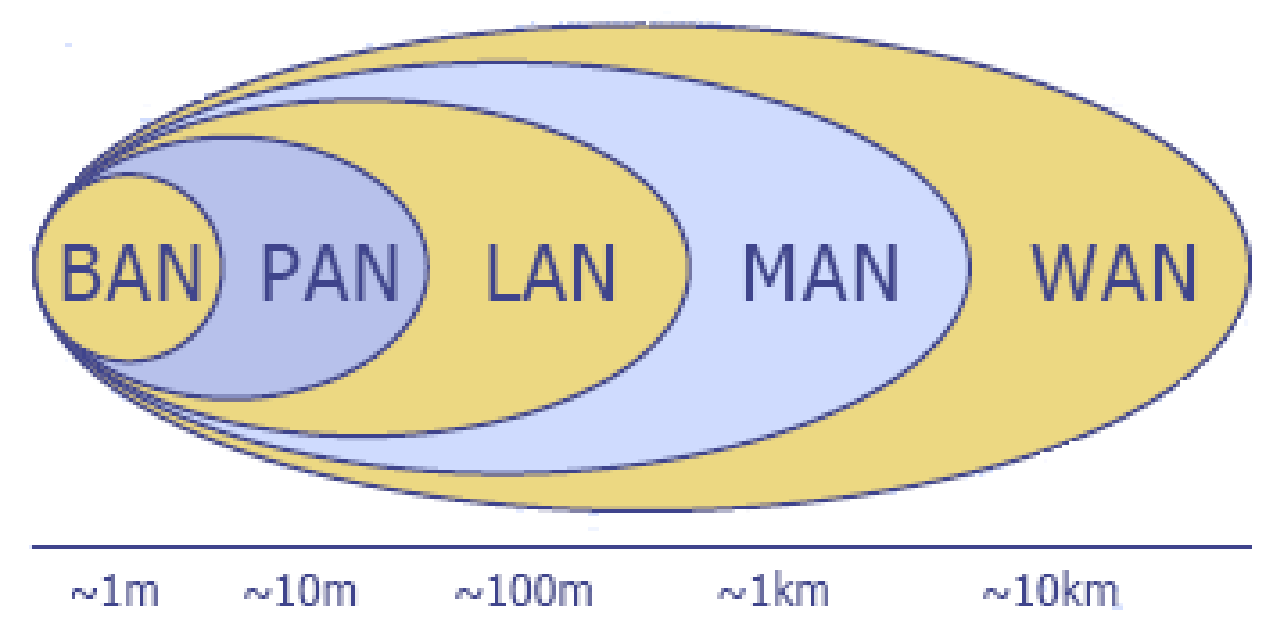

llustración 2.1 Clasificación de redes inalámbricas por área de cobertura

\subsubsection{Redes Inalámbricas Metropolitanas (WMAN)}

Una red de área metropolitana interconecta a usuarios con recursos de red distribuidos en una región mayor que la cubierta por una WLAN, pero obviamente menor que una WWAN. Este término es utilizado para la interconexión de redes dentro de una ciudad para ser consideradas como solamente una entidad, con lo cual se está ofreciendo una conexión mucho más eficiente a las WWAN, o ciertas veces se refiere a la interconexión de diferentes WLAN a los backbones existentes.

Estas redes basadas en infraestructura, permite a los usuarios establecer conexiones de banda ancha entre distintos nodos de la red MAN. Con esta acción se supera lo atinente a altos costos de instalación de las 
redes de fibra óptica y los accesos de cobre. Para la transmisión de datos se utilizan tanto ondas radiales, como del espectro infrarrojo. La IEEE ha creado el grupo de trabajo específico 802.16 [34] sobre accesos inalámbricos de banda ancha, desarrollando estándares y recomendaciones como soporte de la tecnología de las WMANs.

WiMAX (Worldwide Interoperability for Microwave Access) es un nuevo sistema WMAN, capaz de proveer los accesos mencionados anteriormente mencionados a una gran escala. Es la tecnología del momento para el acceso de última milla (ó decenas de kilómetros) tanto para las conexiones de banda ancha fijas ó las móviles.

Por el hecho de ser una tecnología novedosa, existen pocas referencias aún en la literatura [35]. Cabe mencionar aquí que el hecho de pertenecer a WiMAX certifica que el producto en cuestión cumple con los estandartes publicados IEEE 802.16. Estos estándares, compatible con otros anteriores como el de Wi-Fi, permiten interconexiones de ancho de banda similares al ADSL, sin cables; y hasta una distancia de 50-60 kilómetros.

En un análisis socioeconómico de WiMAX realizado en una conferencia de la IEEE [36] se ha establecido que existen nueve factores que afectarán el despliegue actual y futuro de la tecnología, a saber: dispositivos considerados, confusión sobre las virtudes de WiMAX, asignación de espectro radioeléctrico, WiMAX como 4G, WiMAX como tecnología emergente de un país, factores políticos y regulatorios, crisis económicas, competencia WiMAX vs. LTE (Long Term Evolution) [37] , y fundamentalmente quienes son los usuarios finales de WiMAX.

\subsubsection{Redes Inalámbricas de Área Local (WLAN)}

Estas redes, como se infiere de su denominación, permiten a los usuarios establecer conexiones dentro un edificio o un espacio público reducido, pudiendo operar en forma indistinta ya sea en infraestructura, o no. 
En el modo con infraestructura, los nodos inalámbricos se conectan a un punto de acceso (AP) que sirve como bridge entre las estaciones y el backbone de red existente. En modo sin infraestructura, varios nodos inalámbricos son capaces de establecer una red en una determinada zona geográfica, de forma de utilizarla temporariamente. En esa situación no precisan utilizar un AP; aunque tampoco pueden alcanzar recursos externos a la red temporaria establecida. Ejemplos típicos de WLAN son las implementaciones 802.11, conocidas como Wi-Fi e Hiperlan2 [38].

\subsubsection{Redes inalámbricas de Área Personal (WPAN)}

Estas son redes establecidas de manera ad hoc, operando en modo sin infraestructura, y donde vínculos establecidos no superan los diez metros de distancia. Los dispositivos involucrados generalmente son asistentes digitales personales (PDA), teléfonos celulares y laptops que se encuentren en el espacio interactivo considerado, involucrando tecnologías Bluetooth [39], ZigBee [40] y luz Infrarroja [41].

\subsubsection{Redes Inalámbricas de Área Corporal (WBAN)}

Este término es utilizado para describir la portación de dispositivos diminutos, permitiendo con ello la comunicación inalámbrica entre diversos unidades sensores corporales (BSU) y la unidad corporal central (BCU) en el cuerpo humano.

\section{$2.4 \quad$ Redes inalámbricas Celulares}

Estas redes radiales se distribuyen espacialmente sobre áreas denominadas células, cada una de ellas servida al menos por un transceptor conocido como estación base. Cuando estas redes se unen para trabajar en forma conjunta, son capaces de cubrir áreas extensas, 
permitiendo a numerosos transceptores (celulares, pagers, etc.) comunicarse mutuamente, como así también con otros transceptores móviles o fijos, los que pueden estar ubicados en cualquier lugar de la red. La comunicación se realiza por intermedio de las estaciones base.

\subsubsection{Sistemas móviles de Primera Generación}

La primera generación de celulares analógicos fue implementada con AMPS (Advanced Mobile Telephone System) a partir de 1983. La experiencia inicial se realizó en Chicago en un área de 2.100 millas cuadradas, ofreciendo 832 canales con un ancho de banda de diez kilobits por segundo. Las transmisiones desde las estaciones base a los móviles se realiza en una frecuencia entre 869 y $894 \mathrm{MHz}$. El canal inverso está establecido entre las frecuencias de 824 y $849 \mathrm{MHz}$.

En Europa se estableció el TACS (Total Access Communications System) con mil canales y una velocidad de datos de 8 kilobits por segundo. AMPS y TACS utilizan la técnica de modulación de frecuencia para la transmisión radial. El tráfico es multiplexado por el sistema de múltiple acceso por división de frecuencias.

\subsubsection{Sistemas Móviles de Segunda Generación}

Los sistemas $2 \mathrm{G}$ utilizan la tecnología de múltiple acceso digital, como TDMA (Time Division Multiplexing) y CDMA (Code Division Multiple Access). Ejemplos de sistemas $2 \mathrm{G}$ son el GSM, que utiliza la tecnología TDMA para soportar múltiples usuarios; CT2 (cordless telephone), PACS (Personal Access Communication Systems) y DECT4 (Digital Enhanced Cordless Telecommunications). Todos los protocolos $2 \mathrm{G}$ utilizan la codificación digital.

Los protocolos de redes $2 \mathrm{G}$ soportan el transporte de voz y algún otro tipo de comunicaciones de forma limitada, tales como faxes y SMSs. 


\subsubsection{Sistemas móviles $2.5 \mathrm{G}$}

El nacimiento de ésta generación de las redes celulares comenzaron con las redes GPRS (General Packet Radio Service). Esta tecnología radial ha sido ideada para redes GSM; dado que se adiciona el protocolo de conmutación de paquetes, con lo cual mejora la interacción de los proveedores de Internet. Las conexiones GPRS permaneces siempre abiertas, lo que permite a los usuarios de terminales móviles el mismo tipo de disponibilidad de red que la que puede obtenerse en las redes fijas de las empresas. No hay tiempos asociados al establecimiento y liberación de conexiones asociadas. Por ello, las terminales pueden formar parte efectiva de la Internet [42], permitiendo velocidades flexibles de transmisión como también conexiones continuas a la red. GPRS es el paso más significante hacia 3G.

Otra de las evoluciones hacia $3 G$ de estas redes es conocida como EDGE (Enhanced Data Rate for GSM Evolution). EDGE es una técnica de modulación mejorada concebida para aumentar la capacidad de red y las velocidades de datos en las redes GSM, proporcionando una mejora de la velocidad de transferencia de datos sin exigir una nueva infraestructura de red. Se basa en un cambio importante en la norma GSM, para así dar soporte a la modulación de señal basada en la modulación por desplazamiento de fase MDP-8, utilizada actualmente en la modulación GSM [42]. Con esto se permitió a los utilitarios de las bandas GSM utilizar servicios multimedia sobre protocolos internet hasta una velocidad teórica máxima de 384 kilobits por segundo. La implementación de EDGE permitió la competencia y funcionamiento en paralelo y simultáneo con las redes 3G; ofreciendo un servicio similar.

Para ello se requieren mínimos cambios en el hardware y software de red debido a que utilizan la misma estructura TDMA, canal lógico y ancho de banda de transporte que las actuales redes GSM. 


\subsubsection{Sistemas Móviles de Tercera Generación (3G)}

Con estos sistemas se resuelven la mayoría de los desafíos existentes sobre el tema intercomunicación. Aquí deben convivir transparentemente redes cableadas ó inalámbricas, y deben satisfacerse lo referente a ubicuidad.

En Europa tres han sido los sistemas que se han estudiado como redes piloto: UMTS (Universal Mobile Telecommunications Systems) [43], MBS (Mobile Broadband Systems) [44] y WLANs. En el estándar global para la tercera generación de redes de comunicaciones inalámbricas $3 G$ (IMT2000) se establece la utilización de una estructura de celdas jerárquicas. En el año 2000, la Conferencia Mundial de Radiocomunicaciones (CMR-2000) [45] reconoció la creciente importancia de los sistemas de comunicaciones móviles, no sólo para los usuarios de los saturados mercados comerciales de los países desarrollados, sino para un número creciente de usuarios de países en desarrollo en los cuales los sistemas celulares ayudan a satisfacer las deficiencias de los sistemas de acceso fijo. También se asignó espectro adicional a los sistemas en tres bandas mundiales armonizadas, que utilizan atribuciones existentes a servicios móviles y móviles por satélite.

\subsection{Puntos de Acceso (AP)}

Un Punto de Acceso inalámbrico (AP ó WAP) es un dispositivo configurado de manera especial en una WLAN. Este nodo es el que actúa como transceptor principal de las señales radiales en la WLAN. Como tarea normal, los AP soportan estándares de comunicación Wi-Fi.

Si bien las pequeñas WLAN pueden funcionar sin AP en las llamadas redes ad hoc, o en el modo peer-to-peer; los AP permiten acceder a redes en modo infraestructura. El AP generalmente está conectado a una red 
cableada y es capaz de transmitir datos entre dispositivos a cada lado del mismo, es decir en la red cableada y la red inalámbrica.

Estos equipos, de bajo costo y de muy fácil instalación, crecieron rápidamente en popularidad a finales de 1990 y principios de 2000 . Un AP 802.11 antiguo puede comunicarse normalmente a 30 sistemas clientes a un radio de hasta 100 metros. Los equipos de última generación pueden interconectar a 255 clientes.

La distancia especificada puede variar significativamente, dependiendo de variables funcionamiento en exterior o interior, altura sobre el nivel del suelo, obstrucciones físicas, tipo de antena, estado del tiempo, frecuencia de radio utilizada, potencia de salida del equipo, etc.

Para comunicarse, estos dispositivos utilizan un espectro de frecuencias que son legalmente establecidas. Usualmente los AP adyacentes utilizan diferentes frecuencias para comunicarse con sus clientes, y así evitar interferencias entre las dos AP vecinos. Los equipos inalámbricos son capaces de "escuchar" el tráfico de datos en otras frecuencias y con ello pueden cambiar de una frecuencia a otra rápidamente y de esta manera mejorar la recepción desde diferentes puntos de acceso.

En la llustración 2.2 se muestra el esquema de canalización utilizado por la suite de protocolos IEEE 802.11 de manera de evitar interferencias con equipos vecinos [46].

Las redes inalámbricas están en una posición desventajosa si comparamos su rendimiento con respecto a las redes cableadas. En términos de ancho de banda y capacidad de transporte, los equipos inalámbricos comúnmente utilizados en la actualidad pueden alcanzar velocidades de 11 (802.11b) [47] o 54 megabits por segundo -IEEE 802.11a, IEEE 802.11g- [48]. El hardware de red cableado, a iguales costos, permite tráficos a tasas de 1.000 Mbps (Gigabit-Ethernet). 


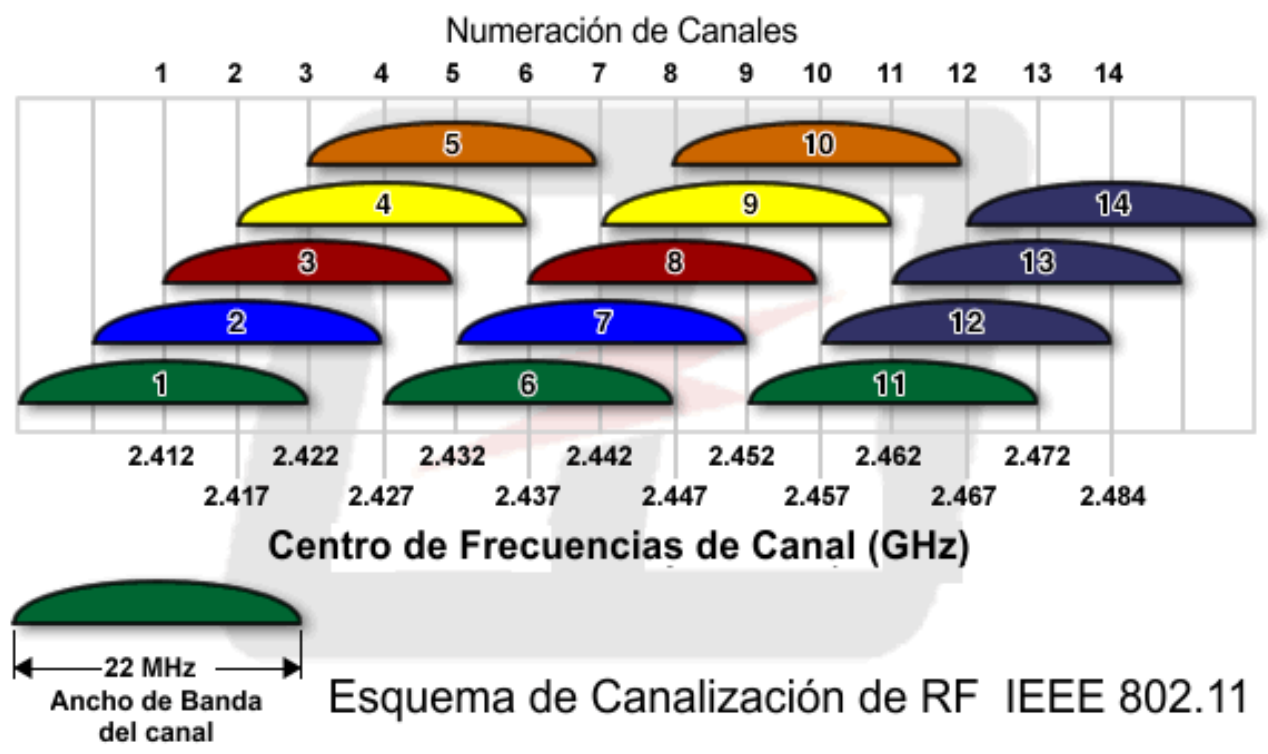

Ilustración 2.2 Esquema de canalización de RF en IEEE 802.11

El éxito de Wi-Fi han impulsado una considerable actividad de investigación, como también ha atraído una inversión importante en el desarrollo de las normas 802.11 , lo que se ha traducido en ampliaciones significativas en la capacidad de la misma. Como resultado, Wi-Fi es cada vez más rápido, más completo y más seguro; al mismo tiempo que los volúmenes de fabricación permiten seguir reduciendo los costos por unidad.

Las mejoras a las normas Wi-Fi permiten abordar nuevos mercados, nuevas aplicaciones y nuevos escenarios de uso. Esta evolución continuará impulsando la adopción de la tecnología, mientras que la mayor inversión por parte de las proveedoras de tecnología conlleva a un mayor crecimiento y madurez de la norma.

La llustración 2.3 muestra la evolución de 802.11 teniendo en cuenta las extensiones aprobadas y por aprobar dentro del grupo de trabajo Wi-Fi. 


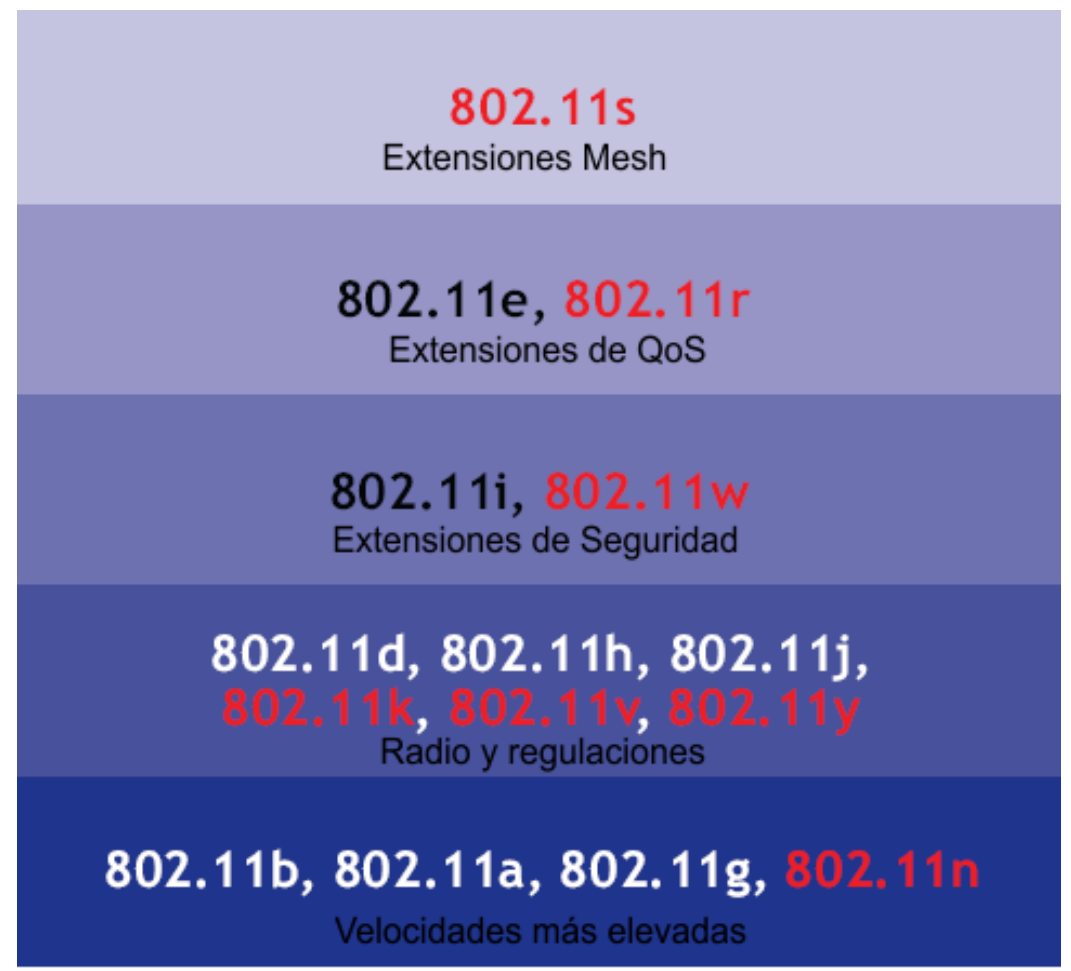

Ilustración 2.3. Evolución de la suite de protocolos 802.11

Uno de los inconvenientes más severos y aún no resueltos en la suite de protocolos 802.11, tiene que ver con la seguridad. Los APs incorporan como herramienta principal una encriptación WEP, que nace en 1999 como un protocolo para redes inalámbricas, el que permite utilizar la criptografía para procesar la información que se transmite en una red WiFi. Su cifrado está basado en el algoritmo RC4, pudiendo utilizar claves de 64 ó de 128 bits.

En 2001 se identificaron una serie de "puntos débiles" en el sistema de encriptación, por donde ha sufrido la mayoría de sus inconvenientes. El "explotar" una clave WEP es cuestión de pocos minutos teniendo el 
software apropiado [49]. La Oficina Federal de Investigaciones de los Estados Unidos de América (FBI) ha demostrado la capacidad de quebrar la clave de encriptación WEP de 128 bits en solamente 3 minutos [50].

La Alianza Wi-Fi anunció en 2003 que WEP había sido reemplazado por WPA. Fue ya en 2004 cuando se ratificó el estándar completo 802.11i (llamándolo WPA2). WPA autentica los usuarios con un servidor, normalmente del tipo RADIUS; y está orientado principalmente a un uso empresarial.

\subsection{Redes Inalámbricas Ad Hoc}

Es de esperarse que la nueva generación de comunicaciones inalámbricas este orientada hacia los usuarios que requieran despliegue rápido de aplicaciones y de telefonía móvil independiente.

Una red inalámbrica ad hoc consiste en un grupo de nodos o terminales autónomas; las que comunicándose unas con otras, forman una red radial multisalto, manteniendo la conectividad de una manera descentralizada [17]. Siendo una comunicación radial, estas redes enfrentan los desafíos e inconvenientes consabidos para este tipo de tecnología. Cada uno de estos nodos es capaz de funcionar como host y router. El control de la red se delega a los nodos en forma distribuida. La topología de la red es dinámica, teniendo en cuenta la alta probabilidad de variación del número de nodos conectados y/o en operación.

Este tipo de redes la conforman las MANET y las WSN. El objetivo fundamental de la primera de ellas es el establecimiento de vínculos entre los diferentes nodos; mientras las WSN son diseñadas de manera de poder detectar o estimar algún evento de interés. Es importante hacer notar y saber que existen características propias de las WSN que no se presentan en las redes ad hoc. Estos son retos presentados como los 
desafíos actuales que precisan de la investigación y desarrollo dentro de las redes ad hoc [51].

Otras de las diferencias existentes entre MANET y WSN, tiene que ver con la cantidad e nodos que toman parte en cada una de ellas. Mientras el tamaño de las redes ad hoc tradicionales alcanza a la decena de nodos; las WSN escalan hasta tamaños de miles de nodos. Generalmente son inmóviles e instalados de una manera densa; por lo cual los mecanismos tradicionales de las redes ad hoc la más de las veces innecesarios, o de un costo operativo elevado. Las WSN tienden a fallar frecuentemente debido a los ámbitos agrestes y remotos de los escenarios donde son instalados, siendo una de las razones (de las tantas) por la que estas redes mudan frecuentemente de topología. Debe tenerse en cuanta también que en una WSN la capacidad cómputo, memoria y energía es sumamente restringida. Los nodos sensores utilizan el paradigma de comunicación por difusión, mientras las MANETS están basadas en comunicación punto a punto. Las WSN pueden requerir alguna información externa para desarrollar las tareas planificadas, por ejemplo datos de localización.

En las redes ad hoc tradicionales existen recursos considerados de forma particular, como el ancho de banda; cosa que no es así en las WSN dado que se espera que los nodos trabajen mancomunadamente, apuntando a un resultado común, normalmente tendiendo a mantener un nivel de calidad de servicio.

Otra característica de las WSN tiene que ver con su característica datacéntrica, es decir capaz de realizar la agregación, compresión, priorizado y descarte dependiendo de la descripción del dato; más allá de la tarea de trasporte de las redes basadas en sus direcciones de red.

En cuanto a la forma de implementar la comunicación entre nodos, en una WSN se realiza por medio de paquetes de datos sumamente cortos, por 
lo que el overhead por el procesamiento de los mismos en cada una de las capas de la red se vuelve muy importante. El modelo de tráfico de las WSN son normalmente de muchos-a-uno, produciéndose el fenómeno conocido de "problema de hot spot" [52] el cual se produce en el nodo de una WSN más cercano a la estación base, dado que deben rutear mayor cantidad de paquetes que los nodos más alejados de la estación base. Por esta razón dichos nodos son primordiales en la vida útil de la WSN.

\subsubsection{Redes Móviles Ad Hoc (MANET)}

Con el avance y desarrollo de los últimos años en las tecnologías inalámbricas, la tecnología ha alcanzado un grado de desarrollo y popularidad que la ha convertido en la preferida de los usuarios en todas las escalas (ilustración 2.4).

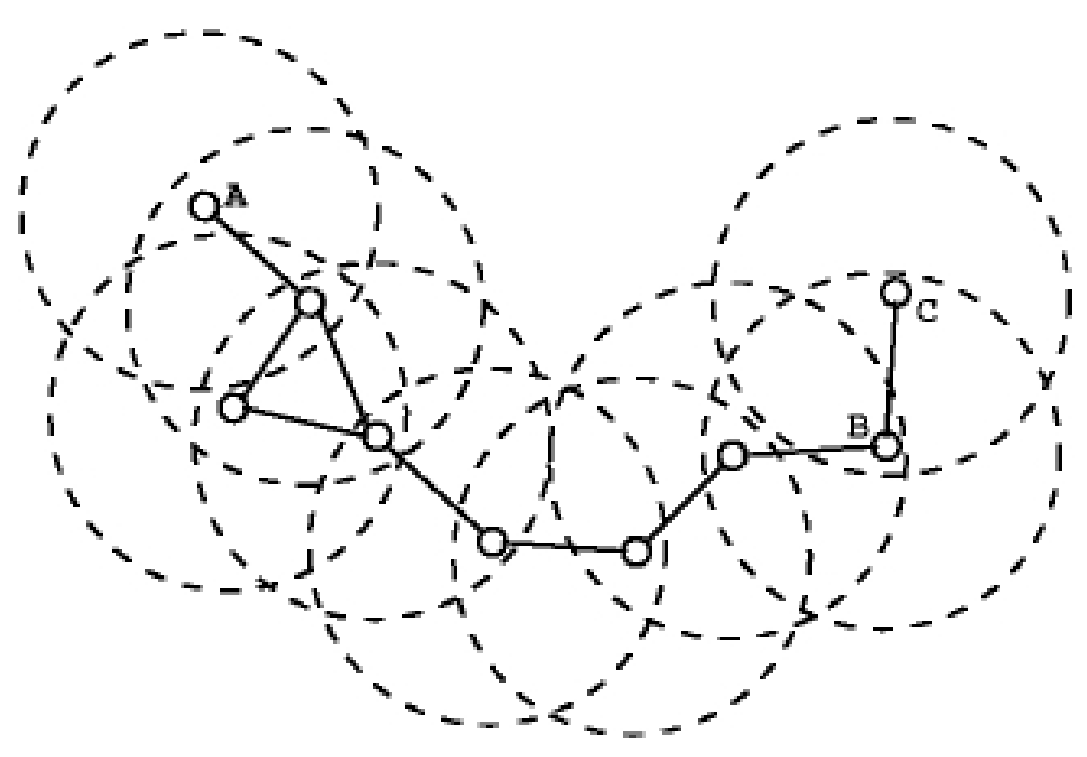

Ilustración 2.4 Esquema típico de red inalámbrica ad hoc 
La misión fundamental de las redes ad hoc es mantener el servicio en función a robustez y eficiencia, parámetros que son función directa de la funcionalidades de ruteo en cada uno de los nodos. Estas redes son muy dinámicas en el tiempo formados por nodos de capacidades restringidas de ancho de banda, cambiando rápidamente de topología en forma aleatoria.

Este tipo de redes ha motorizado el desarrollo y avance de las redes inalámbricas, dado que están directamente relacionadas con el creciente interés de establecer comunicaciones duraderas, eficientes y dinámicas en casos de operaciones de rescate/emergencia y en esfuerzos de colaboración frente a desastres. Pero sobretodo, se destaca la imperiosa necesidad de establecer este tipo de redes en instalaciones militares. En estos escenarios no se puede confiar, o no es posible establecer, una conectividad centralizada y organizada. Son situaciones que ameritan aplicaciones desarrolladas específicamente para MANETs.

La tecnología se ha heredado de las primeras redes Packet-Radio que fueran utilizadas durante los años 1970 y 1980 [53], [54], [55]. En esa época la investigación de este tipo de redes era realizada exclusivamente por los militares en USA, financiadas por el Departamento de Defensa de los Estados Unidos de América (DoD) a través de la DARPA ${ }^{1}$. Estas redes radiales de paquetes fueron las predecesoras directas de Internet, habiendo aportado múltiples colaboraciones a la elaboración de la suite de protocolos originales de IP [56]. Para la década de los 80, la actividad de DARPA incluyó al proyecto SURAN [57] (Survivable Radio Network). La tercera ola de la actividad académica sobre redes inalámbricas ad hoc comenzó en los años noventa, basado en el fenómeno causado por la utilización masiva de placas de radio 802.11, cuyas características

\footnotetext{
${ }^{1}$ DARPA: U.S. Defense Advanced Research Projects Agency
} 
siempre ha sido su economicidad, simpleza de configuración y una capacidad de cambiar rápidamente de escala sin inconvenientes.

Este sistema establece comunicación por medio de paquetes a un canal de radio, y no a un medio cableado. Esta tecnología permite establecer LANs enlazando dispositivos que pueden ofrecer las funciones de puerta de enlace para otros sistemas de red o bases de datos [58]. La versión actual, conocida como Packet Radio Distribuida, permite adaptaciones muy rápidas a los entornos físicos, como también una gran flexibilidad de servicio y fundamentalmente contempla la movilidad de los nodos.

Wi-Fi es capaz de incorporar a los sistemas inalámbricos ad hoc a su esquema, siempre y cuando no estén presentes los AP. En los sistemas 802.11 , cada nodo transmite y recibe datos pero no encamina paquetes entre diferentes redes.

Considerando que las MANET heredan los problemas tradicionales de las comunicaciones móviles e inalámbricas (optimización de ancho de banda, mejora de control de energía y de calidad de transmisión); el diseño de protocolos para este tipo de redes no es una tarea simple. Como norma, se producen interferencias de señal con aquellos equipos terminales que no son destinatarios del mensaje, ya que los canales de comunicación son compartidos por los diferentes equipos inalámbricos. Con ello se produce una caída de rendimiento de las redes a causa de transmisiones simultáneas. La utilización de múltiples canales y/ó múltiples radios puede aliviar el efecto, pero no eliminar definitivamente la interferencia.

La determinación de posibles rutas para la entrega de mensajes en un entorno descentralizado en el que la topología de la red no es fija, es uno de los mayores desafíos para estas redes. Si bien el camino más corto de un origen a un destino es la ruta óptima por lo general en una red estática; esto es de difícil aplicación en las MANET. Diversos factores interactúan afectando al rendimiento del ruteo, como lo son la calidad variable de 
enlace inalámbrico, la pérdida de potencia de señal a lo largo del camino de propagación, la interferencia multiusuario, potencia consumida, y los cambios topológicos [17]. La red debe ser capaz de alterar las rutas para aliviar cada uno de estos efectos. Un caso particular y singular son las redes militares, que son diseñadas para mantener una baja probabilidad de detección, transmitiendo una señal de baja potencia y aleatoriamente.

\subsubsection{Redes de Sensores Inalámbricos}

Existen diversos tipos de sensores, siendo mayoría entre ellos los eléctricos ó electrónicos. Un sensor es un tipo de transductor $^{1}$. Los dispositivos se han vuelto cada vez más sensibles y confiables, desde el momento que se generalizara y expandiera la utilización de MEMS (MicroElectro-Mechanical-System) [59].

La ilustración 2.5 muestra una instalación típica de sensores formando una red. Cada uno de estos nodos posee la capacidad de capturar y diseminar los datos a través de la misma, destinándolos a los sinks (gateways) de la red, dispositivos que se convierten en camino obligado a hacia los usuarios finales.

Todo buen sensor, y que se precie de ello, debe cumplir los siguientes preceptos: ser sensitivo a la propiedad a ser medida, ser no sensitivo a las otras propiedades y sobre todo no debe influenciar a la propiedad medida.

Las redes de sensores son instalaciones que capturan y diseminan datos. Estos equipos fueron desarrollados originariamente en Universidad de California en Berkeley (UCB), denominándolos Mica [6]. Como parte de éstos desarrollos se han desarrollado productos complementarios como

\footnotetext{
${ }^{1}$ Dispositivo electrónico que convierte un tipo energía en otra.
} 
los paquetes de software de código abierto TinyOS [60] y la base de datos TinyDB [61].

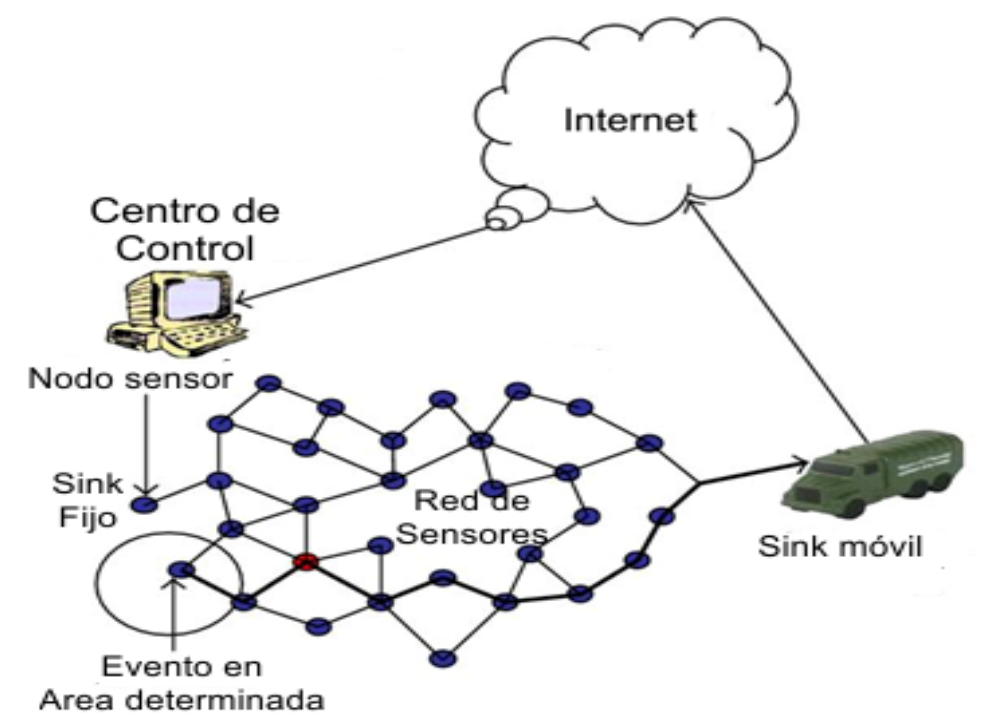

Ilustración 2.5 Instalación típica de nodos sensores en una WSN

Los dispositivos, en una WSN, deben combinar sus puntos de vista sobre los fenómenos físicos dentro de la propia red. Esta cooperación tiene que ver fundamentalmente con la capacidad de permanecer en funcionamiento, su capacidad de almacenamiento y la potencia de cálculo. Cada uno de los nodos está normalmente conformado por un transceptor de radio, un micro controlador y una fuente de energía. Su tarea específica se realiza por medio de sensores adaptados a cada una de las necesidades específicas; pudiendo adicionarse sensores acústicos, sísmicos, infrarrojos, videocámaras, etc.

La mayoría de los equipos de las WSN funcionan con baterías, por lo cual el tiempo de funcionamiento de los nodos es dependiente del tipo y la capacidad de las mismas. La eficiencia energética en estos dispositivos es fundamental, permitiendo con ello prolongar la vida útil de estas redes. 
Para mejorar la calidad de los datos obtenidos de un evento específico, normalmente se procesa y adicionan los resultados a reportes resumidos en cierto número de nodos. Esta fusión de datos requiere la transmisión de paquetes de un tamaño mayor; y de los consecuentes mensajes de control, con lo que la arquitectura de red puede presentar ciertas limitaciones.

Las necesidades de la aplicación pueden requerir que se consulte a todos y cada uno de los nodos de la red, ó a algunos de ellos en cierta región específica. La información requerida por el usuario puede resultar tan grande en términos de tamaño de paquete, que no sea factible trasmitirla por la red. Lo que sí podrían realizar alguno de los nodos intermedios de zonas o regiones, es almacenar esa información en sus recursos propios, para elaborar mensajes resumen conteniendo la misma.

Entonces podemos decir que de las tareas realizadas por las WSNs, a tres de ellas se las considera esenciales: medición, comunicación y cálculo. Cada una de ellas tiene una relación directa con el hardware, software y los algoritmos.

En una WSN cada uno de los nodos puede poseer una dirección global única, en otras se produce un acondicionamiento del dato (calculo) previo a su transmisión.. Un ejemplo típico de las nombradas en primer lugar se encuentra en una playa de estacionamiento, donde la red sensores debe ser capaz de indicar los espacios libres disponibles.

La agregación de datos como medida de mejoramiento de la calidad de servicio, puede ser contraria las necesidades de la aplicación y utilización de la red. Pensemos en el caso de detección de intrusos, evento que debe ser inmediatamente comunicado a la autoridad de manera de aplicar alguna de las políticas establecidas. 
Por último; otro de los desafíos de esta tecnología es la interoperabilidad. Siendo que este tipo de dispositivos tienden a ser cada vez más económicos, que el tipo de comunicación establecida no precisa dedicación, y de los continuos avances en las comunicaciones inalámbricas, es de esperarse que en un futuro no muy lejano el despliegue de este tipo de redes sea una tarea sumamente común. Como consecuencia de ello, será muy probable el escenario donde convivan equipos de diferentes marcas, proveedores y/ó características.

\subsection{Alcance y tasas de transferencia}

En la ilustración 2.6 se presenta para cada una de las tecnologías presentadas en este capítulo, el rango de su utilización y los picos máximos de transferencia.

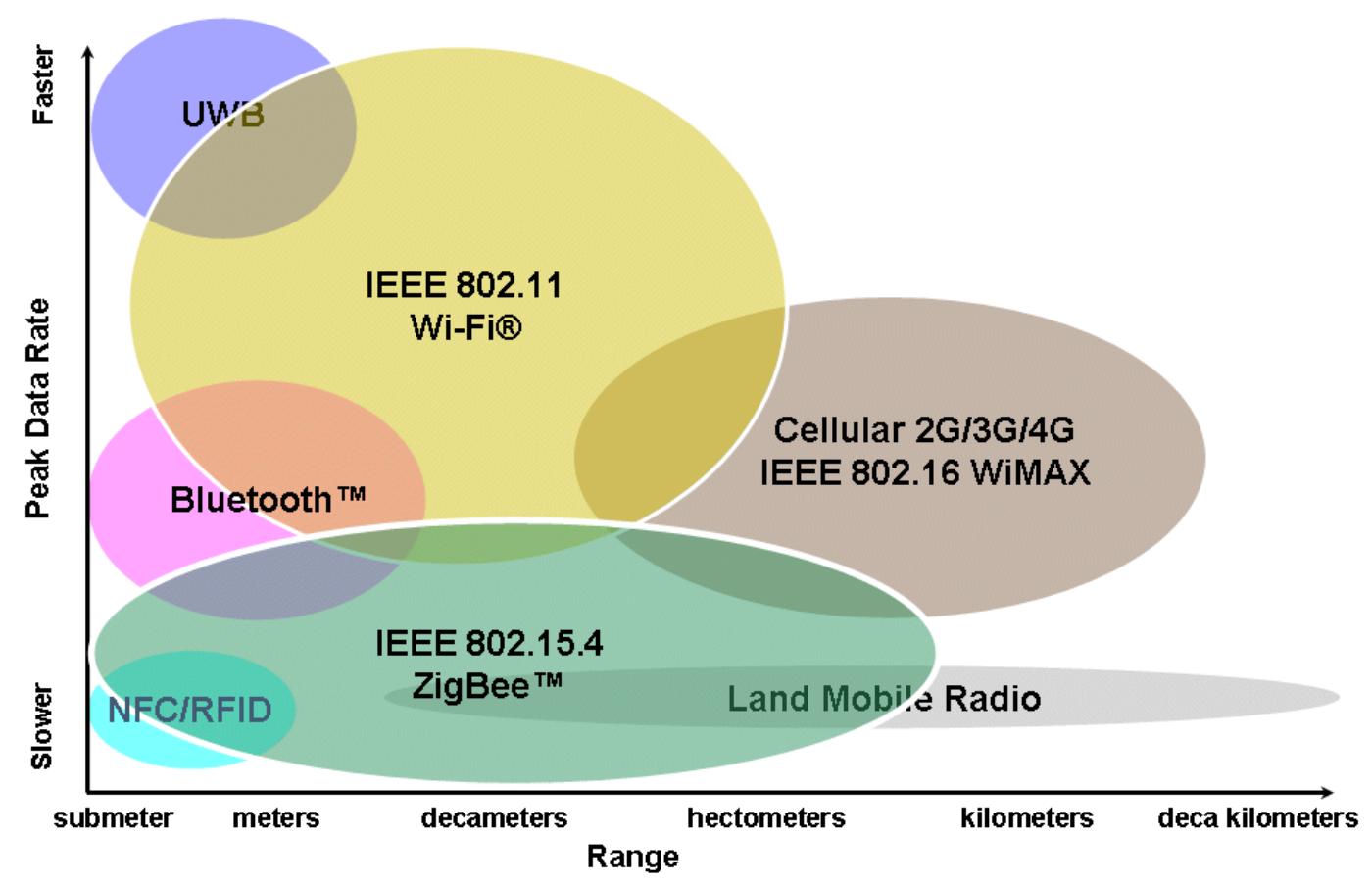

llustración 2.6 Alcance y tasa máxima de transferencia de las diferentes tecnologías [62] 


\subsection{Resumen}

Las comunicaciones inalámbricas se han vuelto omnipresentes. El número de teléfonos móviles y los usuarios inalámbricos de Internet se han multiplicado significativamente en los últimos años.

Las redes de primera generación fueron enfocadas a las comunicaciones de voz, y algunos datos a velocidades bajas de transferencia. Con la llegada de $2 G$ y $3 G$, se incorpora a las comunicaciones inalámbricas las características conocidas como banda ancha. Aparte de orientarse como soporte de movilidad, la banda ancha está orientada también hacia el tráfico multimedia, incluyendo con ello calidad de servicio (QoS).

En este capítulo se han presentado las diferentes tecnologías inalámbricas, y considerado la necesidad de la interoperabilidad.

Se han presentado ejemplos de sistemas de segunda generación como el GSM, (CT2), de Acceso Personal Sistemas de Comunicación (PACS), y Digital Europea teléfono inalámbrico (DECT). Las WLAN se han presentado como tecnologías que extienden a las redes cableadas actuales. Se han revisado en este capítulo el estándar de IEEE para redes inalámbricas, tales como WWAN, WMAN, WLAN, WPAN y WBAN. 
Capítulo 3 : Redes de Sensores Inalámbricos 
i bien los nodos sensores han sido utilizados desde hace décadas en aplicaciones tan diversas como terremotos y el ámbito castrense; el desarrollo evidenciado por esta tecnología ha sido impresionante a partir de 1998 con el proyecto Smartdust [63], [64], [65]. El proyecto se presentaba como una tecnología emergente, notable por utilizar diminutos equipos que conformaban, en definitiva, una pequeña computadora. Incluían fuente de energía, sensores y un sistema de comunicación. El objetivo era desarrollar la tecnología hasta lograr que cupieran en un milímetro cubico de volumen. Si bien es cierto que ya finalizado el proyecto no se ha alcanzado el tamaño meta mencionado, el proyecto ha catalizado y disparado innumerables trabajos sobre el tema en todo el mundo.

Las redes de sensores inalámbricos, proveen una tecnología que permite operar de forma autónoma a cada uno de los nodos, sin depender de infraestructura alguna. Las WSN son parte de aquellos objetos cooperantes, que residiendo en la zona de la computación ubicua; permite desarrollar una gran variedad de aplicaciones prácticas. La computación ubicua es un modelo de interacción de personas y equipos, en los cuales el procesamiento ha sido asimilado invariablemente a los elementos y actividades del día a día. Es de prever que, en un futuro próximo, las WSN formen parte indivisible de nuestro medio ambiente. Cada uno de los objetos con los cuales interactuamos todos los días, tienden a ser integrados con sensores de alguna naturaleza, siendo desarrollados estas políticas por la estimulación de diversos elementos de la industria y organizaciones gubernamentales.

Tomando en consideración el tráfico de datos que generarían en las redes globales todos estos nuevos dispositivos realizando mediciones sobre fenómenos determinados en ambientes hasta ahora insondables, el volumen del tráfico global se incrementaría de manera alarmante, significando esto un cambio arquitectónico de la infraestructura general de 
las tecnologías de la Información. Ante este escenario, es donde el procesamiento de datos en unidades centralizadas sería convenientemente reemplazado por el agregado y el filtrado de datos en crudo.

La ilustración 3.1 muestra el escenario futuro próximo que se espera en el ámbito de las WSN.

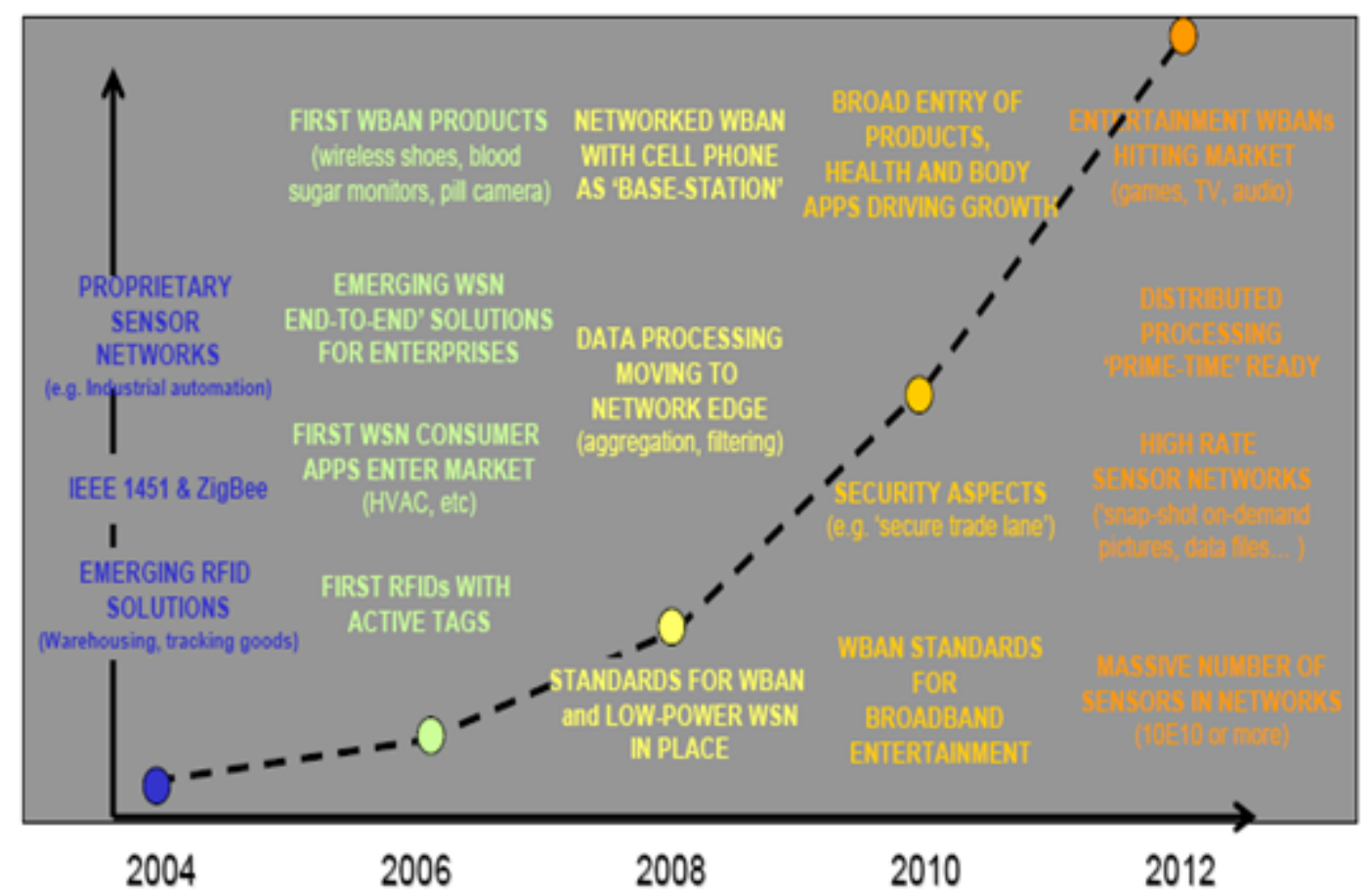

Ilustración 3.1 Proyección estimada de la penetración de las WSN en la sociedad

Los avances alcanzados en la tecnología de las redes inalámbricas en general, nos han colocado en la puerta de una nueva era, en la cual pequeños equipos inalámbricos nos proveerán acceso a la información en cualquier momento $\mathrm{y}$ en cualquier lugar, permitiéndonos asimismo participar activamente en la creación de "espacios inteligentes" [66]. Uno de estos espacios inteligentes son las WSN. Estas se establecen de modo ad hoc con el objeto de medir algún parámetro de un fenómeno 
determinado. Esta tecnología promete ser revolucionaria por su confiabilidad, exactitud, flexibilidad y facilidad de instalación.

Las WSN representan una mejora considerable cuando se compara con la utilización tradicional de los sensores. La utilización de éstos últimos normalmente se realiza de alguna de las siguientes maneras [67]:

- Los sensores se colocan lejos de la ocurrencia del fenómeno, utilizando lo que se conoce como sentido de la percepción.

- Se instalan varios sensores que solamente realizan la medición.

- Como regla, transmiten los valores obtenidos del fenómeno a un lugar específico donde los datos son procesados y reportados.

Una WSN en tanto, se compone de una gran cantidad de equipos (nodos), que generalmente son del mismo tipo. La función de un nodo individual es obtener un valor (dato), y que el mismo sea reconocido como válido y por lo tanto sea procesado. Para ello estos nodos deben reaccionar de manera particular ante ciertos eventos, reconociéndolos, pre-procesarlos y finalmente aprobarlos. Cada uno de estos equipos, es capaz de interconectarse inalámbricamente con equipos similares por medio de protocolos de ruteo multisalto.

La diferencia paradigmática fundamental entre las WSN y las otras redes inalámbricas, se encuentra en que la comunicación en las WSN no se realiza de extremo a extremo.

Al vincularse los nodos sensores entre sí, forman un sistema complejo que es mucho más potente; siendo capaz de realizar tareas más complicadas que aquellas llevadas a cabo por un nodo de manera individual. Normalmente, cada unos de los nodos de una WSN posee las siguientes características: poca velocidad de procesamiento, exiguo almacenamiento, estrecha banda de comunicación, poca memoria y corta 
vida útil. En consecuencia los dispositivos de una WSN poseen una gran capacidad de procesamiento en su conjunto, pero no por separado; y sobretodo son equipos de muy bajos recursos computacionales.

El utilizar sistemas autónomos cooperantes para la realización de diferentes tareas es algo común en ciertos entornos. Los primeros fueron los instalados en el sistema militar antisubmarino Sound Surveillance System (SOSUS) [68], el que fuera complementado luego con el Sistema Integrado de Vigilancia Antisubmarino (IUSS) [69].

Diferenciemos un sensor y un actuador. Los actuadores son casi tan diversos como los sensores, sin embargo, son más simples. En principio, todo lo que un nodo sensor puede hacer es abrir o cerrar un interruptor o un relé o definir un valor de alguna manera. Si esto controla un motor, una lámpara, o algún objeto físico no es realmente de interés para el diseño de los protocolos de comunicación entre los sensores. Esa tarea es exclusiva del actuador. Una buena práctica de diseño es tener en cuenta que en la mayoría de las aplicaciones se debe relacionar siempre un actuador con un sensor de control, siguiendo el principio de "nunca confiar solamente en un actuador" [70].

En las WSN se identifica claramente al sensor como una interfaz entre el observador y el fenómeno que se quiere investigar. El sensor es el equipo que realiza la determinación en el fenómeno investigado y realiza el reporte de las medidas. El usuario final, interesado en obtener información sobre el fenómeno que la red de sensores analiza y disemina, es el observador. Éste puede indicar su interés en algún tipo particular de dato efectuando consultas específicas, a las cuales la red sabrá responder apropiadamente. El fenómeno es la entidad de interés del observador, y que está siendo investigada por intermedio de la red de sensores.

El interés del observador (nivel de aplicación) está centrado en la información obtenida y debe ser transmitida por la red de sensores. La 
información se analiza como un todo en el sistema distribuido de captura de datos. Como dijimos, lo más importante para el observador es el fenómeno, no interesándose de la WSN subyacente. La consulta se lleva a cabo como uno, o más, de los intereses específicos de bajo nivel

Las redes de sensores comparten muchos de los retos de las redes inalámbricas tradicionales. Uno de ellos tiene que ver con la energía necesaria para su adecuado funcionamiento, por ende, con la vida útil de la misma. Otro de los elementos a tener en cuenta son el limitado ancho de banda disponible, y la siempre existente posibilidad de errores en los canales de comunicación utilizados.

Es conveniente que realicemos un pequeño análisis del porque el desarrollo de equipos utilizados en la informática han confluido en el desarrollo de la idea de las WSN.

Hacia 1997, Mark Weiser quien es considerado el padre de la computación ubicua; se planteaba los siguientes interrogantes “¿Cuál es la idea de la computadora del futuro? ¿El agente inteligente?, ¿La televisión (multimedia)?, ¿El mundo gráfico 3-D (realidad virtual)? ¿El equipo de voz ubicua de la película StarTrek?, ¿Las interfaces gráficas de usuarios -GUI-, elaboradas y seleccionadas?, ¿La máquina que por arte de magia cumpla y acceda a nuestros deseos?" [71]. La respuesta que él mismo ha dado es: ninguna de las anteriores. La sencilla razón es que todos estos conceptos comparten un defecto básico: se llevan a la práctica con algo tangible, visible.

Al inicio de este trabajo, hemos reproducido una frase de Weiser indicando que "las tecnologías más significativas son aquellas que se mimetizan entretejiéndose en la trama de la vida cotidiana hasta que se tornan indistinguibles de la vida misma". Toda herramienta que se precie de ser útil, debe ser ubicua, considerándolas así a aquellas que no involucren conscientemente a la persona, centrándose fundamentalmente 
en la tarea; no en la herramienta. Los anteojos son una buena herramienta, ya que nos fijamos en el mundo, no en las gafas. El ciego tocando con su bastón siente la calle, no al bastón en sí mismo. Por supuesto las herramientas no son invisibles, sino que forman parte de un contexto de utilización de las mismas. Con suficiente práctica, hasta podremos hacer desaparecer multitud de cosas, que si bien las utilizamos muy frecuentemente, nuestra memoria las ha olvidado hace largo tiempo. Las buenas herramientas realzan a la invisibilidad.

Este campo de estudio ha venido a llamarse la "Computación Ubicua". Es algo completamente diferente a las notebooks, netbooks, PDAs; o a la información al alcance de la mano. Es invisible, es la computación en cualquier lugar; pero que no es residente ni dependiente de un dispositivo personal.

\subsection{El Desarrollo Tecnológico y la escala}

El hecho de la desaparición física de la computadora personal ha sido predicha hace muchos años, aunque se observa que el mercado de los mencionados equipos es todavía vital y estable, siendo fuente de frecuentes y revolucionarias innovaciones. Con el paso de los años el procesamiento de la información se ha ido distribuyendo a dispositivos cada vez más pequeños y descentralizados.

La ilustración 3.2 clasifica al equipamiento informático tanto por su costo como por el grado de conectividad alcanzado por cada uno de ellos a lo largo de los años [72], [73]. En el ángulo inferior izquierdo se encuentra uno de los primeros equipos que se vendiera como mainframe, la IBM $\mathrm{S} / 360$. La conectividad en los años de 1960 no era tema relevante, dado que se realizaban cálculos simples y operaciones binarias para lo cual era más que suficiente 64 kilobytes de memoria. 


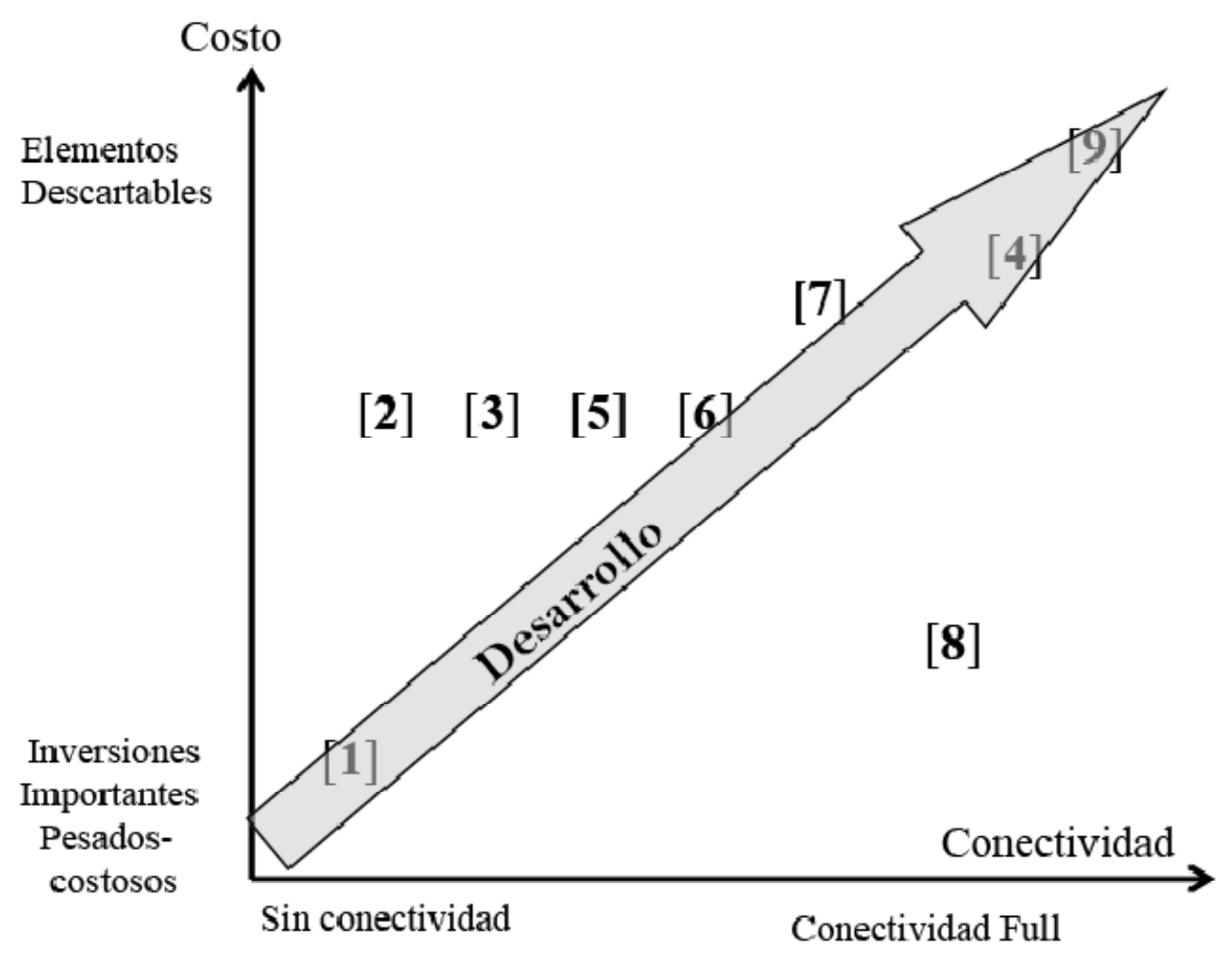

Ilustración 3.2 Clasificación de computadoras: [1] IBM S/360, [2] Apple II/IBM PC AT/XT, [3] PCs/Amiga 486, [4] Teléfonos celulares a granel, [5] PCs Pentium

[6] PCs de la era "siempre encendidas", [7] PDA con GPRS, [8] Automóviles conectados, [9] Redes de Sensores

Un cambio de paradigma se produjo en los años 1970-1980 cuando se asumió que cada uno de nosotros deberíamos poseer una computadora personal. Las primeras PC fueron producidas por Apple, Texas Instruments e IBM.

Si bien en esos años se utilizaban módems para efectuar comunicaciones digitales sobre líneas telefónicas analógicas; llevó casi 10 años que esta tarea se volviese rutinaria. El método de conexión por modem fue adoptado por las empresas como forma de conectividad; como un paso previo a la existencia de Internet. 
La diseminación y migración de las computadoras personales de las corporaciones y grandes agencias gubernamentales hacia las pequeñas y medianas empresas, y a los usuarios privados, podría ser interpretado como un primer paso en el largo camino existente hacia los equipos descartables y de bajo costo; los que se ubican al final de la escala.

Para finales de los años 1980, ya con la visión de productos orientados a la conectividad, hacen su aparición los teléfonos celulares; los que se producen a gran escala. El éxito de la telefonía celular ha demostrado la necesidad de las personas de permanecer continuamente interconectada; y liberarse de las conexiones cableadas y estaciones de trabajo fijas. El límite físico existente entre PDAs y los equipos celulares se va esfumando día a día.

Los modernos automóviles son capaces de intercomunicarse, compartiendo entre ellos la información referente accidentes, embotellamientos, problemas en el camino, etc. Los pasajeros, pueden revisar sus correos electrónicos, consultar la información turística del sitio a visitar; mientras se entretienen con audio o video digital. En un futuro próximo, y dado que los automóviles no son económicos, ni descartables; estaríamos en presencia de las redes de sensores más importantes en la escala establecida anteriormente.

Podemos en este instante redefinir a las WSN como un sistema de diminutos equipos autónomos, que cooperan; en casi en todas las aplicaciones, de manera de resolver al menos una aplicación común. Su tarea incluye siempre algún tipo de percepción de parámetros físicos.

El término cooperar debe entenderse como transmitir la información. En el fenómeno físico de transmitir la información de forma inalámbrica se presenta como inevitable la utilización de antenas, elemento que no encaja cuando encuadramos a la tecnología como formada por equipos diminutos. 


\subsection{Características de las WSN}

Las WSN comparten ciertas características generales con las redes ad hoc. Por lo tanto el diseño de protocolos para WSN deberán considerar a las propiedades de éstas redes.

Se deben tener en cuenta la limitada vida útil de los equipos por la imposibilidad de los nodos de obtener fuentes externas de energía, la comunicación inalámbrica no confiable por naturaleza, y la característica de autoconfiguración con poca o nada de intervención humana.

\subsection{Escenarios de Aplicación}

Las WSN son muy útiles en ocasiones en las que la observación humana es casi imposible, o cuando los sistemas de detección por cables son difíciles de instalar, o su costo es prohibitivo.

En la ilustración 3.3 [74] se muestra una aplicación típica para una red de sensores, realizando el monitoreo de penetración de la humedad en un dique destinado a combatir las inundaciones por crecidas de un río. Con estas instalaciones es posible informar a los equipos de socorro con datos en tiempo real sobre el estado del dique, informando de filtraciones ó defectos en las defensas por la existencia de pequeñas filtraciones en las estructuras.

Los nodos sensores se incorporan a bolsas de arena donde se efectúa el control de humedad. Una vez instalados los sensores, éstos establecen una red ad hoc espontánea. Las lecturas de cada uno de los nodos, húmedos o secos, se comparan con las mediciones de los nodos vecinos, de manera de evitar falsos positivos o negativos. 


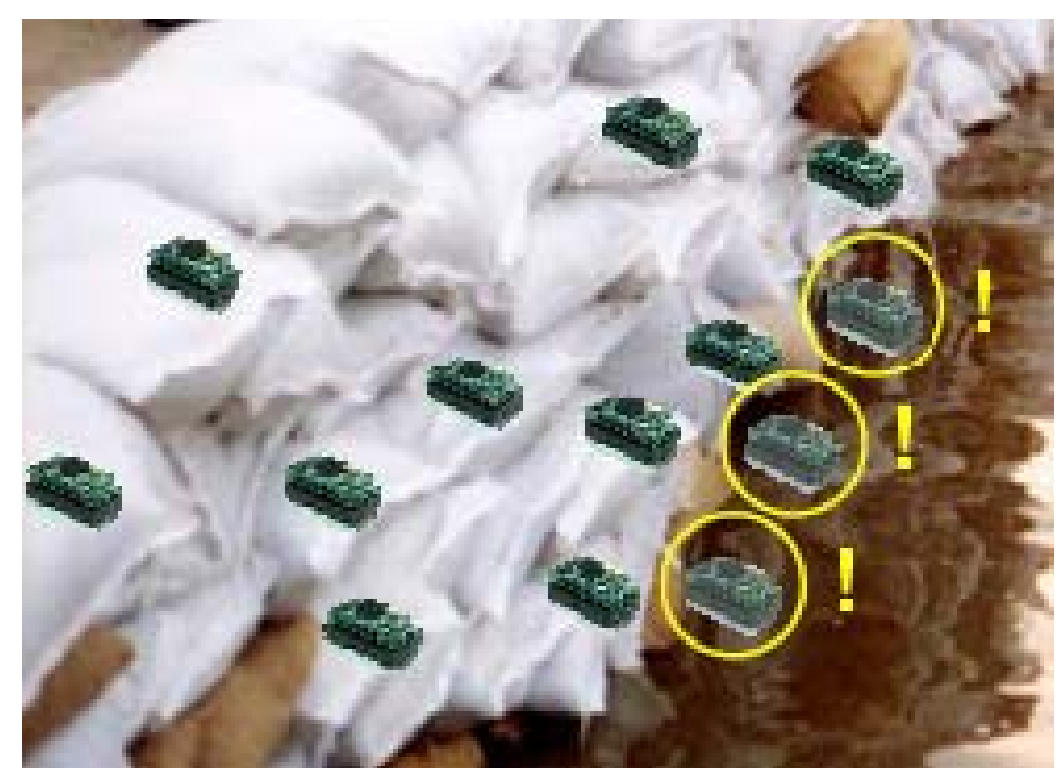

llustración 3.3 Nodos sensores en diques de contención

Luego de esta etapa de coordinación local, la lectura del sensor es enviada a uno o más pasarelas. En la ruta a un nodo controlador (sink), las lecturas de los distintos sensores son comparadas y procesadas, de manera de no transmitir información errónea.

El monitoreo de hábitats es una de las tareas insignias de las WSNs. Esta nueva tecnología permite a zoólogos y biólogos realizar el seguimiento de distintas comunidades sin realizar ninguna perturbación en el ambiente investigado.

Entre los años 2003 y 2004, se han desplegado sensores en los nidos bajo tierra de petreles en Great Duck Island, donde las aves procrean [75], [76]. La ilustración 3.4 muestra un diagrama de la red instalada, mostrando un conjunto de nodos que ha medido el microclima de los distintos nidos (temperatura, humedad, presión barométrica y radiación infrarroja). Otros nodos proporcionaron información sobre las condiciones fuera de los nidos. Los datos colectados fueron enviados por medio de 
una red multisalto a la central de manera de establecer una base de datos.

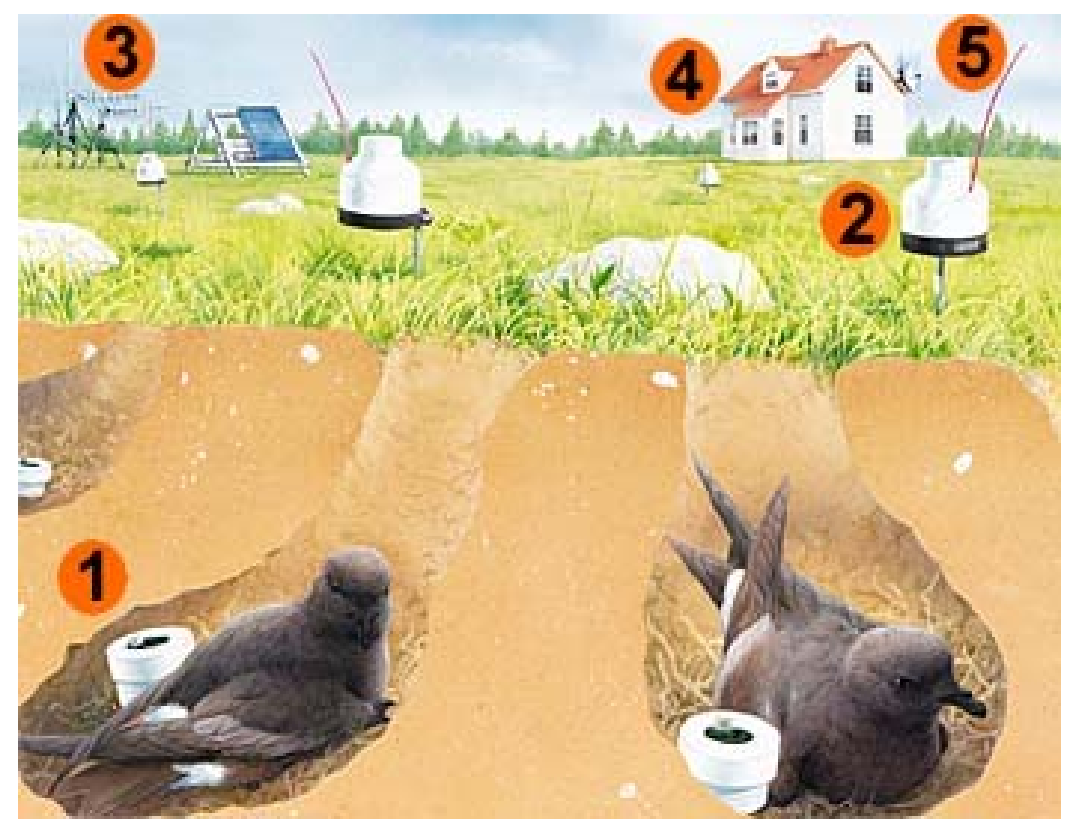

Ilustración 3.4 Monitoreo de petreles en la isla Great Duck

En el sistema ZebraNet [77] se ha utilizado GPS [78] para registrar datos precisos de la posición de diferentes animales (cebras), para así controlar las migraciones de estos a lo largo del tiempo. Siempre que los sensores portados por cada animal se encontraren dentro del rango de rango de comunicación de otros equipos similares, por ejemplo cuando abrevaban por un determinado tiempo, se realizaba el intercambio de información sincronizando la misma con todos los nodos, de manera que cada animal almacene toda la información de la red.

Se han realizado innumerables controles en ambientes naturales, donde se ha buscado observar la evolución de ciertos parámetros considerados de interés. Ejemplo es el estudio realizado sobre el comportamiento y 
emisiones en el volcán Tungurahua, Ecuador [79]. Para ello se ha instalado una WSN en el volcán durante el mes de Julio de 2004. La red ha capturado y almacenado señales infra sónicas a $102 \mathrm{~Hz}$, y ha transmitido esta información a una estación base remota ubicada a 9 kilómetros. Se realizaron mediciones durante 54 horas continuas, intervalo en el cual se experimentaron 9 eventos muy importantes (explosiones). Los nodos han sido sincronizados utilizando GPS. En la ilustración 3.5 se muestra el arreglo de sensores realizado en campo [80].

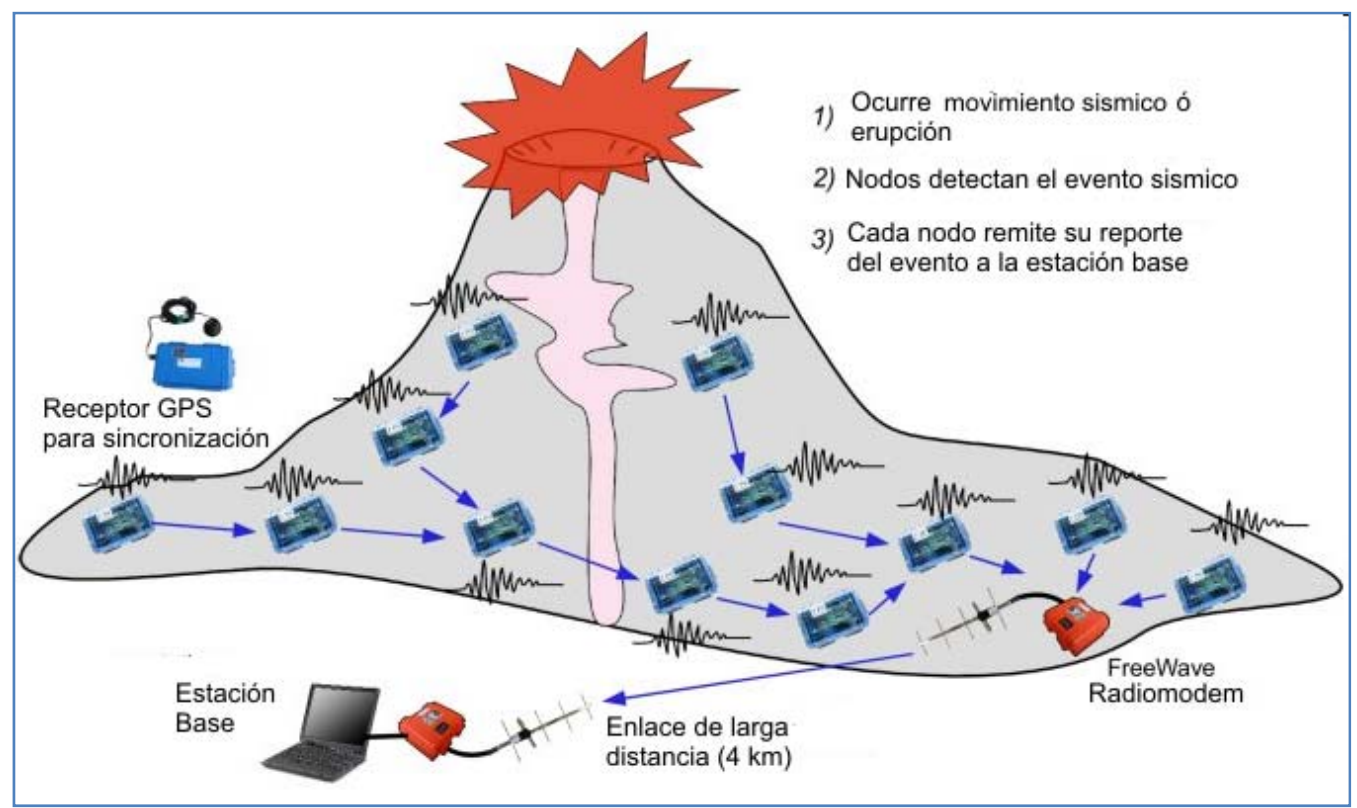

Ilustración 3.5. Arreglo de Sensores para monitoreo volcánico

Frente a la crisis del calentamiento global, de la escasez de agua, y las demandas de una economía ágil; los agricultores y productores están recurriendo a soluciones WSN para así de ahorrar en los costos laborales, aumentar los rendimientos, mejorar la calidad, y conservar el recurso agua. En zonas de California y Australia donde se presentan condiciones de sequía recurrentes, conjuntamente con los altos precios de la 
irrigación, los productores apenas pueden mantener el ritmo de la demanda. En el año 2008 se realizó una encuesta, determinándose que de 36 vitivinicultores y agricultores, más de la mitad eran usuarios de sensores inalámbricos; y casi un tercio estaban planeando nuevas aplicaciones inalámbricas de sensores. Como parte de esta acción Accenture Technology Labs instaló una WSN en los viñedos Pickberry [81] en un área de 30 acres, de manera de medir continuamente humedad relativa ambiente, velocidad del viento, humedad del suelo y temperatura del aire. Esta información de transmitía de manera inalámbrica a una PC ubicada en las oficinas. Esta es una herramienta valiosa para los productores, ya que son capaces de determinar la mejor manera de planificar y manejar sus cultivos.

Un caso de éxito de las WSN en control sanitario de la población ha sido Health Smart Home [82], establecida en un entorno diseñado por la facultad de Medicina de Grenoble (Francia), entorno donde Intel ha realizado una experiencia de instalación de 130 nodos de control en individuos localizados en una residencia geriátrica. Los dispositivos eran portados por los diferentes internos, controlando los datos fisiológicos de los pacientes, rastreando a médicos y personas en los edificios, efectuando asimismo la administración de fármacos.

En el proyecto MarathonNet [83], [84] una red de sensores es utilizada para monitorear los eventos durante una maratón. Los corredores voluntarios reciben un nodo sensor especial (pacemates) que controlan el ritmo cardíaco captado por una correa a nivel del pecho. Esta información es emitida a diferentes estaciones base a lo largo del recorrido. Por medio de diferentes tecnologías las estaciones base se conectaba con el servidor central, equipo en el que se almacenaban los valores sensados de cada corredor. Los espectadores han podido seguir la maratón en tiempo real, mientras que los organizadores han controlado el ritmo 
cardíaco de los corredores; pudiendo alertar a los servicios médicos ante cualquier eventualidad de riesgo en la salud de alguno de ellos.

La domótica también utiliza a los sensores de manera de poder establecer "edificios inteligentes" [85] para mejorar la calidad de vida de los usuarios y ahorrar energía en el edificio por medio de un monitoreo distribuido. La mayor parte del consumo diario de energía se debe a los consumos propios de los edificios donde habitamos. En un esfuerzo por economizar los escasos combustibles fósiles en la tierra, las redes de sensores son una herramienta valiosa para incrementar la eficiencia energética de los edificios, sin que ello afecte severamente nuestra calidad de vida. Un edificio de oficinas podría ser receptor de cientos de sensores de niveles de intensidad luminosa y temperatura, como también de movimiento. La WSN puede ser capaz de encender ó apagar las lámparas de los distintos ambientes si existiera detección de movimiento. De la misma manera la WSN ajustaría la temperatura de la climatización, relevando de esta tarea a los humanos.

En el monitoreo de salud de estructuras (SHM), deben tenerse muy en cuenta dos factores en la programación de las tareas: la escala de tiempo y la severidad del cambio. La escala de tiempo indica la rapidez a la que un cambio tiene lugar, y la severidad se corresponde con el grado cambio ocurrido. Existen asimismo dos categorías de SHM, la respuesta a desastre (terremoto, explosión, etc.) y el monitoreo de carácter continuo (vibraciones ambientales, viento, etc.). El método convencional es el realizado con computadoras interconectadas a acelerómetros piezoeléctricos por medio de cables [86]. Entre las desventajas de los métodos cableados podemos mencionar la necesidad de instalar los cables por toda la estructura, pudiendo afectar la misma; y el costo de los equipos es alto; donde el costo de instalación es un factor a tener en cuenta y por último es necesario un mantenimiento de costo elevado. Comparado con los métodos convencionales, una WSN proporciona la 
misma funcionalidad a un precio mucho más bajo, por lo que permite aumentar el número de los puntos de vigilancia. En la ilustración 3.6 se puede observar la distribución de nodos sensores efectuada en el puente Golden Gate para realización de SHM.

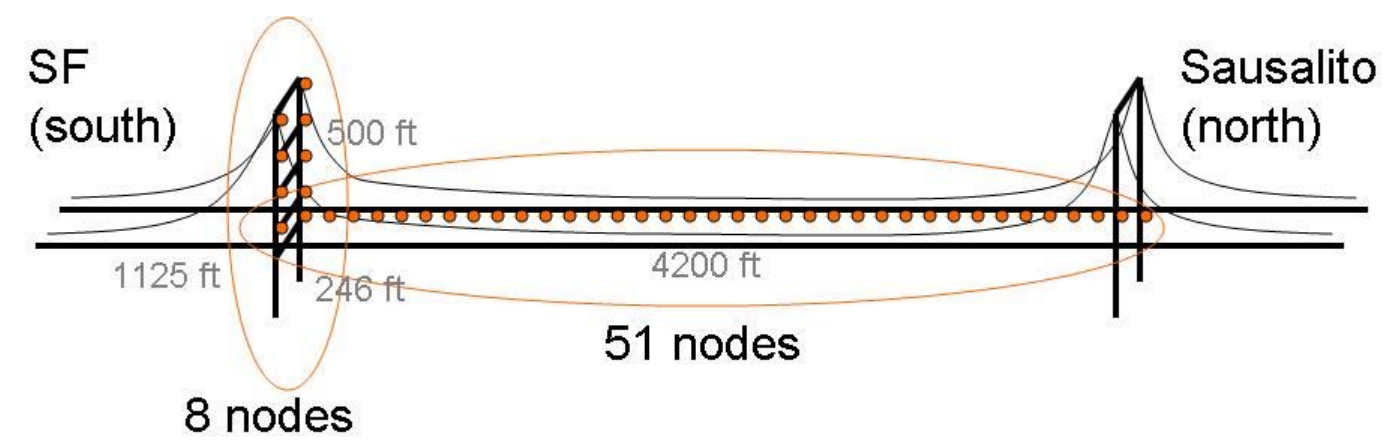

llustración 3.6. Distribución de nodos para SHM en el puente Golden Gate [87]

En otro campo de utilización de la tecnología, y ya en el ámbito castrense, encontramos al campo de minas Self-Healing [88]. Estas minas son capaces de determinar si su condición en un determinado momento es activada o inerte, estado que deviene de acuerdo a la característica de la serie de eventos ocurridos. Normalmente las minas antitanques son colocadas conjuntamente con minas antipersonales para evitar el desarmado de las mismas. Este nuevo tipo de arma es un obstáculo independiente y dinámico, que responde a la acción de evitarlas con una reacción de reordenamiento de los nodos, como se observa en la ilustración 3.7. Los nodos pueden moverse a una velocidad de hasta 1 $\mathrm{m} / \mathrm{s}$. Una vez instalado el campo minado, éste comienza a realizar el control de la integridad del obstáculo, realizando la vigilancia anti brecha por medio de la comunicación mina-a-mina. Cuando el enemigo ha producido una brecha en el campo minado, o se ha hallado un camino por 
el cual es posible vulnerar a la instalación, los nodos tienen capacidad de moverse y auto organizarse, evitando con ello los corredores liberados [89].

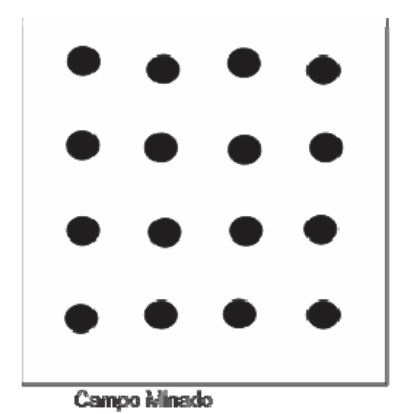

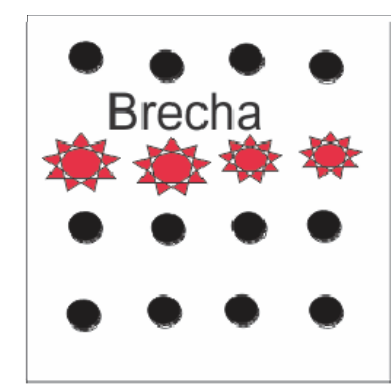

Brectiva groducicia

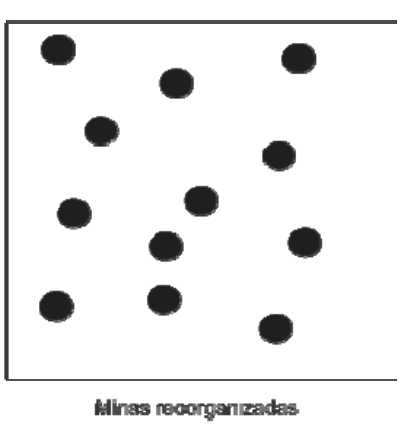

Alinas necorgenzadas

Ilustración 3.7. Auto organización de minas Self-Healing

\subsection{Factores de diseño de las WSN}

El diseño de las WSNs están influenciadas por diversos factores [67], entre los cuales podemos nombrar la tolerancia a fallas, la escalabilidad, los costos de producción, el ambiente de operación, la topología de la WSN, las limitaciones de hardware, el medio de transmisión, y el consumo energético. Estos factores son importantes, ya que son utilizados como guía para el diseño de los protocolos y algoritmos de las WSNs.

\subsection{Métricas de evaluación de las WSN}

Analicemos los parámetros considerados como primordiales al momento de evaluar una WSN.

En primer lugar debemos conocer ciertamente que ventajas posee esta tecnología sobre otras existentes y que uso daremos a la red. Los factores a considerar son la vida útil, cobertura, costo, facilidad de 
implementación, tiempo de respuesta, certeza temporal, seguridad y velocidad efectiva de muestreo. El resultado de estos análisis presentara siempre un grado de interrelación que no puede evitarse. Normalmente debe sacrificarse uno de los parámetros, de manera de poder obtener una mejora de rendimiento en otro. Por ejemplo, el disminuir el número de muestras de los eventos, es una manera de aumentar la vida útil de la red.

Tomado todos los puntos establecidos por las métricas, se conforma el espacio multidimensional que debe usarse para caracterizar a una red de sensores inalámbricos. Una aplicación específica de este tipo de redes, representa solamente un punto en ese espacio. La plataforma seleccionada será evaluada positivamente, si es capaz de cumplir con los requisitos de la aplicación dentro del hiperespacio que muestra la capacidad de tareas del equipo.

\subsubsection{Vida útil}

Es el elemento crítico en la evaluación de las WSN. El fin práctico de una red, con referencia a la vida útil, consiste que una vez instalada la WSN, esta funcionará de manera autónoma por largos períodos de tiempo, desde varios meses hasta años.

La primera limitación es el modo en el que se provee la energía necesaria para el funcionamiento de los nodos. La tecnología ha avanzado en éste campo de la ciencia muchísimo más que la tecnología de las baterías utilizadas para mantenerlas funcionando. Consideremos la evolución de la potencia de cálculo y la memoria por unidad de superficie de cada uno de los nodos y podremos dimensionar esta disociación entre la evolución de la tecnología inalámbrica y el de las baterías. 
En los equipos GPS portátiles, en las cámaras digitales compactas, en computadoras personales de bolsillo; las baterías ocupan un importante porcentaje del volumen. La realidad indica que la energía provista por las baterías en estos dispositivos es escasa, considerando que el equipo suele salir de servicio en el momento que más lo necesitamos.

¿Por qué de la ocurrencia de este fenómeno? Es muy simple de ser explicado. Las baterías siguen siendo pequeñas latas de componentes químicos, que funcionan de igual manera que lo hacían hace dos siglos, a partir del experimento de Alejandro Volta. La tabla 3.1 indica los capacidad de cada tipo de batería, potencialmente utilizables por las WSN en un futuro próximo [90], [75].

\begin{tabular}{lc}
\hline Tecnología de la batería & Watt-hora/gramo \\
\hline Batería química de iones Litio & 0,3 \\
Celdas combustibles de Metanol* $^{*}$ & 3,0 \\
Batería nuclear de Tritio** $^{*}$ & 850,0 \\
Batería nuclear de Polonio-210** & $57.000,0$ \\
\hline
\end{tabular}

Asumiendo $50 \%$ eficiencia - ${ }^{* *}$ Asumiendo $8 \%$ de eficiencia y 4 años de operación

Tabla 3.1 Potencial energético para diferentes tecnologías de baterías

En una WSN cada nodo debe realizar su tarea con el objetivo de maximizar la vida útil de la red, no importando la suya propia. En algunos escenarios es posible utilizar la energía provista por fuentes externas a los sensores, es decir de alguna infraestructura existente. Sin embargo, una de las características que hemos nombrado como ventaja de las WSN es su facilidad de instalación.

La pregunta clave de toda instalación es la siguiente: ¿Cuál es la energía disponible y cuál es el grado de utilización de la misma? Los equipos 
deben poseer suficiente energía almacenada, para mantenerse activos por largos períodos de tiempo. Caso contrario, deberán ser capaces de almacenar energía por alguno de los métodos conocidos de aprovechamiento de energías alternativas.

Hoy se conocen muchas técnicas de generación y almacenamiento, mientras que solamente algunas de ellas son utilizadas. Algunas de estas técnicas son extravagantes, y rendimientos muy bajos. Los más conocidos son los estudios realizados utilizando celdas solares [91], y generadores piezoeléctricos [92], [93].

En las tareas de campo solamente 4 técnicas son ampliamente recomendadas por su rendimiento en entornos prácticos: a) diferencia de temperaturas b) fotocélulas (interior y exterior) y c) radiofrecuencia.

La tabla 3.2 muestra una lista de los valores estimados e producción de energía por los diferentes métodos indicados anteriormente [94].

En los nodos se presenta el consumo máximo de energía durante la transmisión y recepción de datos. Esto debe considerarse cuando se pretende aumentar la duración de las baterías por medio de una disminución de la potencia radial del nodo, ó disminuyendo los ciclos de transmisión a un mínimo. Ambas alternativas se corresponden a diferentes sacrificios en las métricas del sistema.

Dietrich y Dressler [95] realizan un análisis detallado y profundo sobre las distintas metodologías y parámetros de medición de la vida útil de las WSN.

\subsubsection{Cobertura}

Conjuntamente con la vida útil, la cobertura es de las métricas fundamentales al momento de evaluar una WSN. La facultad de poder 
desplegar la red en una gran superficie, representa siempre una ventaja significativa.

Tabla 3.2 Potencia generada por diferentes fuentes alternativas

\begin{tabular}{|c|c|}
\hline Fuente de Energía & $\begin{array}{c}\text { Potencia } \\
\text { Generada }\end{array}$ \\
\hline Vibración- Movimiento & \\
\hline Humano & $4 \mu \mathrm{W} / \mathrm{cm}^{2}$ \\
\hline Industrial & $100 \mu \mathrm{W} / \mathrm{cm}^{2}$ \\
\hline Diferencia de Temperaturas & \\
\hline Humana & $25 \mu \mathrm{W} / \mathrm{cm}^{2}$ \\
\hline Industrial & $1-10 \mathrm{~mW} / \mathrm{cm}^{2}$ \\
\hline Luz & \\
\hline Interior & $10 \mu \mathrm{W} / \mathrm{cm}^{2}$ \\
\hline Exterior & $10 \mathrm{~mW} / \mathrm{cm}^{2}$ \\
\hline Radio Frecuencia & \\
\hline GSM & $0,1 \mu \mathrm{W} / \mathrm{cm}^{2}$ \\
\hline Wi-Fi & $0,001 \mu \mathrm{W} / \mathrm{cm}^{2}$ \\
\hline
\end{tabular}

Es importante saber diferenciar entre la cobertura de la red y al alcance de la red inalámbrica. Las técnicas de comunicación multihop pueden extender la red mucho más allá del alcance propio de la tecnología de radio utilizada. En teoría, la red se podría extender indefinidamente. Pero para un rango dado, la red multisalto incrementa el consumo de energía de los nodos, atentando contra la vida útil de la red. Además, esto requiere de una densidad de nodos mínima, aumentando los costos de instalación y distribución. 
Cuando consideramos la cobertura, sabemos que se debe considerar la escalabilidad de la WSN, es decir su capacidad de interaccionar con un gran número de nodos. La escalabilidad es una de las proposiciones de valor de las WSNs. En nuestra experiencia, hemos instalado una pequeña WSN como prueba, red a la cual se le han adicionado diferentes nodos a los largo del trabajo; pudiendo además adicionar diferentes nodos sin solución de continuidad. Con ello aumentamos la capacidad de la red de toma de muestras, como también podremos diversificar el tipo de información capturada.

En ciertas oportunidades la red puede alcanzar 10.000 o incluso 100.000 nodos. Por ello deben ser necesariamente auto-organizadas, escalables, tolerantes a fallos, con procesamiento distribuido de datos, etc. Si bien alguno de los nodos puede llegar a desconectarse del resto de la red, se debe asegurar un alto grado de conectividad por medio un alto grado de redundancia.

La densidad de sensores en cada proyecto es muy variable. Este valor se calcula con la siguiente ecuación

$$
\mu(R)=N \pi P^{2} / A
$$

Donde $\mathrm{N}$ es el número de sensores en el área $\mathrm{A}$ y $\mathrm{R}$ el alcance de transmisión de radio. El valor de $\mu(\mathbf{R})$ representa el número de nodos que se encuentran dentro del rango de transmisión de cada nodo en la región considerada.

La escalabilidad en WSN debe considerarse integralmente tanto en el hardware como en el software. Para el hardware, escalabilidad implica sensibilidad y alcance de los sensores, ancho de banda y uso de la energía. Referente al software podemos asociar a la escalabilidad con la fiabilidad de los comandos de difusión y transferencia de datos, gestión de 
grandes volúmenes de datos y la utilización de algoritmos escalables para el análisis [96].

El usuario normalmente confía en la capacidad de escalamiento de la red proyectada de acuerdo a las necesidades del proyecto. Pero debe considerarse que el aumento del número de equipos conlleva a un aumento de las transmisiones necesarias, con lo que se aumenta el consumo energético global de la WSN. Una alternativa es realizar mediciones no tan frecuentes del parámetro investigado, y así disminuir el número de transmisiones.

Establecer alternativas para estas situaciones permite la aplicación de WSNs más allá de prototipos de laboratorio, replicándolos a una escala mayor, como la vida real.

\subsubsection{Tolerancia a fallas}

Debe considerarse que el comportamiento de las WSN es impredecible, y que generalmente se producen fallas en ellas. Estas fallas pueden generar zonas ciegas dentro de la WSN, debido al aislamiento de un cierto número de sensores. Esto puede llevar a comportamientos anormales de la red, y hasta situaciones de colapso total de extensas redes.

Algunos sensores pueden fallar, ser robados, dañados, interferidos físicamente, o simplemente agotar la energía de sus baterías. Esto no debería afectar el rendimiento en las tareas de la WSN. La tarea de reemplazo de baterías, ó de algunos equipos, suele ser una tarea complicada en redes extensas, o cuando la geografía de la zona de instalación es complicada. Una WSN debe ser tolerante a fallas, y así asegurar el desarrollo de las tareas asignadas a la misma [97]. 
Tolerancia a fallas la definimos como la capacidad de mantener la funcionalidad de la red, es decir sin interrupciones, a pesar de la salida de funcionamiento de algunos nodos [98], [99]. La confiabilidad $\mathbf{R}(\mathbf{t})$ ó también llamada tolerancia a fallas, puede ser modelada utilizando la distribución de Poisson [98], representando la probabilidad de no fallar en un intervalo de tiempo [0,t] [98],

$$
R(t)=e^{-\lambda t}
$$

Donde $\lambda$ y $\mathbf{t}$ son la tasa de falla del sensor y el período de tiempo considerado. Como resultado de esto, el nivel de la tolerancia a fallas de una WSN dependerá del tipo de aplicación, y de todos los posibles esquemas de fallos que se planteen para una instalación determinada.

Se puede considerar la tolerancia a fallos en cuatro niveles diferentes de abstracción, a partir del hardware y software del sistema $e$ ir al middleware y las capas de aplicación [97]. En algunos estudios, se considera la tolerancia a fallas en cada uno de los componentes individuales de un nodo. Por último se puede considerar la tolerancia a fallos de cada nodo a nivel de red.

\subsubsection{Costo y facilidad de instalación}

La ventaja fundamental de las WSN respecto a otro tipo de tecnologías tiene que ver con la facilidad de instalación. Demás está decir que los biólogos, ó los obreros de la construcción, poco saben de los mecanismos subyacentes de comunicaciones y de redes de datos cuando se encargan de realizar seguimientos de ciertos fenómenos por medio de una WSN.

Una WSN exitosamente instalada y puesta en marcha, debe ser capaz de auto configurarse. Cada uno de los nodos puede distribuirse en la zona de estudio por personas que no han sido entrenadas para la tarea específica. El sistema debe simplemente funcionar bajo cualquier modo de 
configuración física. Por supuesto que siempre existen limitaciones en los sistemas reales, como la imposibilidad de instalar nodos de alcance infinito.

La WSN debe ser capaz de evaluar y determinar problemas potenciales en la red. Debe descubrir enlaces y determinar la calidad de los vínculos establecidos. Sumado a ello cada uno de los nodos deben adaptarse inmediatamente a la variabilidad del medio ambiente en la de la zona de instalación. Una de las premisas de toda instalación es evitar la obstrucción de la línea de visión entre los nodos; aunque los equipos deben reconfigurarse automáticamente para poder superar este inconveniente.

El costo de instalación y configuración es importante en la primera etapa del ciclo de la vida útil de una WSN. A largo plazo, los costos mayores se producen por la necesidad de mantenimiento de los elementos componentes de la red.

El escenario particular de aplicaciones en donde se considera a la seguridad, se requiere que el sistema sea sumamente robusto, además de una concienzuda selección y consideración de software y hardware. La red debe ser capaz de realizar una rutina de auto mantenimiento, debiendo poseer algunos mecanismos de alarma, en caso que sea necesaria la presencia física de equipos técnicos para realizar ciertas tareas específicas.

En las instalaciones reales, parte de la energía disponible en el sistema se utiliza para las tareas de verificación y mantenimiento, reduciendo con ello la vida útil de la red, generando tráfico de diagnósticos y reconfiguraciones. Esto disminuye, obviamente, la tasa efectiva de recolección de información. 


\subsubsection{Tiempo de respuesta}

Considérese el caso típico de disparo de una alarma. Ante estos eventos la celeridad es la mejor consejera, tanto para asistencia física en el lugar del evento, como el reporte a los responsables de responder ante la mencionada situación. Se instalan las alarmas e implementan los métodos de notificación de manera de dispararse justamente en el momento de intrusión. Si bien en cada uno de los nodos rige la regla de uso racional de la energía de manera de prolongar la vida útil de la red, debe existir una prioridad de envío de ciertos mensajes tan pronto como se produzca el evento. Como hemos dicho anteriormente la vida útil de la red es mayor cuanto menor sea la utilización del equipo de radio.

Ante ciertas circunstancias el tiempo de respuesta de las WSN podría mejorarse si dejáramos los equipos activos continuamente, logrando con ello que los mensajes sean distribuidos inmediatamente, ó de acuerdo a las prioridades establecidas para cada uno de los eventos a detectar.

\subsubsection{Precisión Temporal}

Para ser precisa temporalmente, la WSN debe ser capaz de mantener una base de tiempo global que pueda ser utilizada, cronológicamente, para ordenar las muestras y los eventos. Parte de la energía utilizada por las redes se destina a la mantención del reloj distribuido. La sincronización que se realiza por medio del envío de diferentes mensajes, debe ser continuamente transmitida entre nodos, dependiendo la precisión deseada de la mayor frecuencia de mensajes. Por lo tanto queda claro que el mantenimiento de reloj distribuido en una WSN requiere en primer lugar energía, y fundamentalmente de disponibilidad de ancho de banda. 
En aplicaciones medioambientales, de seguimiento o de monitoreo; las muestras puntuales no son de mucha importancia. La tarea normalmente consta en que las muestras obtenidas por los diferentes nodos deben ser comparadas y correlacionadas de manera de obtener un valor real y así propagar los valores medios del fenómeno.

En el caso de medición de temperaturas en un edificio, las muestras serán suficientemente representativas si realizamos la medición dentro de los segundos, aunque podría considerarse realizar las mediciones tras varios minutos. Cuando se evalúa la reacción del edificio frente a eventos sísmicos, ineludiblemente es imperativa una notificación dentro de los milisegundos.

\subsubsection{Privacidad}

Muchas veces se sospecha que las corporaciones, gobiernos o individuos no tendrían el menor pudor en utilizar los nodos de una WSN para poder vigilar comportamientos de otros individuos sin su consentimiento previo, en un acto puramente de espionaje. En general todas las redes pueden ser utilizadas con fines espurios, ilegales, poco éticos. Las WSN no son la excepción a la regla.

No es tarea simple avanzar con la implementación de la privacidad en las WSN. Pero considerando que las WSN cada día son más económicas, pequeñas y muy difundidas en la sociedad, se visualizan inconvenientes reales en lo que se refiere a límite éticos y morales de la utilización de estas redes.

\subsubsection{Seguridad}

La seguridad en las WSN es un tema que cobra importancia a medida que las aplicaciones experimentales de las mismas están migrando hacia 
la industria. El adicionar un gran número de dispositivos capaces de generar información y tráfico consiguiente, los que se sumaría a los que existen al momento; claramente contribuirían a generar a un caos generalizado. La seguridad, no contemplada en los albores de Internet, es para las redes ad hoc uno de los elementos que condicionan su destino exitoso.

La temperatura, intensidad luminosa o humedad relativa son valores puntuales que, obtenidos por un nodo meteorológico; no poseen mucha importancia aparentemente. Sin embargo el mantener la seguridad de estos datos es sumamente importante. Utilizando estos datos es posible realizar estudios sobre el comportamiento de las variables temperatura y luminosidad de un determinado edificio. En manos no deseadas, estos valores pueden ser utilizados en la planificación de ataques estratégicos, económicos o físicos a una empresa u organización.

Con el desarrollo y aplicación de WSNs a distintas facetas de nuestra vida cotidiana, la seguridad de la información conforma un valor específico muy importante; especialmente cuando las redes tienen utilización de uso militar [100].

Con la autenticación de usuarios y dispositivos se asegura que los actores en el proceso de comunicación son legítimos y que son quienes dicen ser. La autenticación de los mensajes permite verificar la integridad de los datos. La protección de la integridad de los datos tiene por finalidad prevenir el intento malicioso de corromper o modificar los datos que son portados por un mensaje. Diferentes personas ó dispositivos podrían estar interesados en obtener información de la WSN. Por ello deben proponerse, como normalmente se realiza en otras redes, mecanismos de autenticación como una medida de protección ante ataques internos y externos. 
La utilización de la criptografía en una red WSN se utiliza para el aseguramiento de los vínculos establecidos, para asegurar los servicios distribuidos localmente y tolerar adecuadamente la captura de nodos diversos [101]. Las tareas que no pueden realizarse ó sustituirse con la criptografía son: prevenir el análisis de tráfico, eliminar paquetes retransmitidos, prevenir la reutilización de paquetes, prevenir sobre el arribo de paquetes retrasados, prevenir los embotellamientos y evitar capturas de nodos de la WSN con intenciones espurias. Como se observa, los procedimientos criptográficos no son algo mágico para convertir un servicio inseguro en uno seguro.

Las técnicas criptográficas ayudan, pero no son la solución final para el inconveniente. Una de las alternativas en el diseño de WSN a fin de evitar inconvenientes frente a nodos capturados con fines espurios, es apuntar a la robustez de la WSN. En las WSN, pueden implementarse diferentes tipos de seguridad en diferentes niveles del sistema. Cualquier técnica de protección es válida, una de las más difundidas es la llamada marca de agua [102].

Para cualquier infraestructura Ad hoc deben considerarse requerimientos de seguridad en protocolos de enrutamiento, autenticación del acceso a través de la administración de claves, y sistemas de detección de intrusos.

\subsubsection{Tasa Efectiva de Muestreo}

Definimos a este parámetro como la velocidad a la que un dato, tomado por un nodo cualquiera, puede ser comunicado al punto central de almacenamiento de la información.

En algunas aplicaciones se necesitan tasas efectivas de muestreo de 10 2 muestras por minuto. Sin embargo, conjuntamente con la velocidad de 
cada nodo individual, debemos considerar el impacto de las arquitecturas de red multisalto sobre la capacidad de las mismas de retransmitir la información desde nodos vecinos.

En un árbol de captura de datos, un nodo debe manejar los datos de todos sus descendientes del árbol considerado. Si cada uno de los nodos transmite su lectura propia y a la vez posee 30 descendientes, se verá necesitado de transmitir 30 veces los datos y a su vez recibir las 30 lecturas en un determinado período de tiempo. Este incremento multiplicativo en la comunicación de datos, incrementa notablemente los requerimientos del sistema.

Uno de los mecanismos más utilizados para incrementar la tasa efectiva de muestreo, hasta más allá de la capacidad original de la red, es aprovechar la capacidad de procesamiento de la misma. Se pueden utilizar métodos de compresión espacial y temporal, reduciendo con ello el ancho de banda de la comunicación, mientras se mantiene la misma tasa efectiva de muestreo.

En las WSN es un comportamiento normal el utilizar el almacenamiento local a altas velocidades durante cortos períodos de tiempo. El procesamiento interno de los datos en la red puede establecer cuando ha ocurrido un evento importante [103], y automáticamente disparar el almacenamiento de datos. Los datos pueden luego ser descargados sobre la red multisalto con la rapidez que lo permita el ancho de banda disponible.

\subsection{Componentes de Hardware}

En la ilustración 3.8 se pude observar un esquema de elementos componentes de un nodo sensor. 


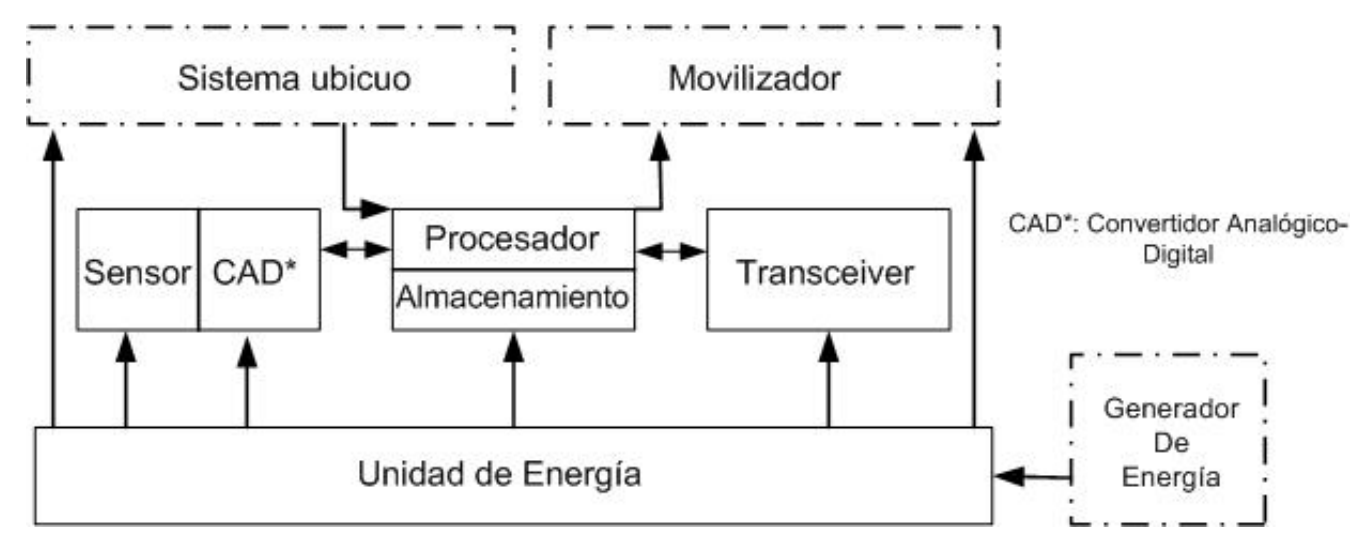

Ilustración 3.8. Vista general de los componentes de un sensor

- CPU: Procesa todo lo referente a aplicación, y es capaz de ejecutar código arbitrario. Generalmente es asociada con una pequeña unidad de almacenamiento. Es responsable de manejar los procedimientos de un sensor, y de colaborar con los otros nodos para realizar las tareas específicas. Es conocido también como unidad microcontroladora (MCU)

- Sensores y Actuadores: es la interfaz al mundo real, los equipos son capaces de observar y/ó controlar parámetros físicos del ambiente. Traducen el fenómeno investigado en señales eléctricas.

- Memoria: Los nodos precisan de algún tipo de memoria para almacenar datos intermedios y/ó programas. Usualmente son distintos tipos de memoria los utilizados para programas que aquellos para datos.

- Provisión de Energía: Tiene que ver fundamentalmente con la utilización de alguno de los tipos de baterías existentes en el mercado. 
- Transceptor: puede ser un dispositivo óptico pasivo o activo, ó un equipo de radiofrecuencia (RF). Los primeros precisan de la línea de visión entre equipos, razón por la cual han sido sucesivamente reemplazados por los segundos.

En relación directa en la aplicación que ha sido prevista para el nodo, puede considerarse la utilización de otros componentes tales como GPS, generador de Energía y unidad de movilización.

Se catalogan a los sensores en tres grandes categorías [104].

- Sensores pasivos omnidireccionales: miden una magnitud física en la ubicación del nodo mismo, sin interaccionar con el medio ambiente que lo rodea (pasivo). Sin embargo, estos tipos de sensores son capaces de autoalimentarse de energía del ambiente por alguno de los métodos estandarizados. Esta energía es utilizada para la amplificación de sus señales analógicas. En estas mediciones no existe noción de dirección. Ejemplo de éstos tipos de sensores son los termómetros, sensores lumínicos, de vibración, micrófonos, de humedad, tensión en materiales, sensores químicos sensibles a algunas sustancias determinadas, detectores de humo, presión atmosférica, etc.

- Sensores pasivos de haz estrecho: Son pasivos como los anteriores, pero tienen bien definida la noción de medidas y direcciones. Un caso típico es una video cámara que puede efectuar mediciones de parámetros en una determinada dirección.

- Sensores activos: Estos últimos realizan un muestreo activo del ambiente, por ejemplo un sonar, radar, sísmico, etc.

Las WSN están compuestas por un gran número de nodos, los cuales son dispuestos ya sea "en" el fenómeno a ser medido ó controlado; ó muy 
cerca de él [67]. La utilización de una WSN refuerza la idea del esfuerzo mancomunado de cada uno de los nodos. La ubicación de los sensores no se calcula o predetermina, por lo que pueden instalarse zonas inaccesibles; ó devastadas.

Habiendo considerado las métricas a tener en cuenta al momento de evaluar los diferentes rendimientos en las WSN, consideremos ahora los parámetros que califican a los nodos individuales.

\subsection{Métricas de evaluación de nodos individuales}

Los factores de diseño de los nodos individuales que consideraremos sirven de guía para el diseño de los protocolos y algoritmos de las WSN. Algunos de los parámetros son similares a los utilizados en la evaluación de las redes en sí mismas.

\subsection{Energía}

Para que las aplicaciones que ejecutan los diferentes nodos individuales sean capaces de funcionar por periodos adecuados y calculados como vida útil de la red (meses, años), la demanda energética de cada uno de estos equipos debe ser, indefectiblemente, muy baja. Los sensores son equipos dependientes de baterías, siendo ésta la razón de su delicado balance de energía. El reemplazo de las baterías no es una tarea factible cuando se considera una red de varios miles de nodos.

Las técnicas de diseño de baja energía y las diferentes estructuras de hardware solo proveen una solución puntual, que ha demostrado ser insuficiente para los sistemas de WSN que se caracterizan por ser energía-limitante.

A diferencia de los teléfonos celulares donde el consumo promedio de la energía se mide en cientos de miliamperios y vidas útiles de varios días, 
el consumo energético en un nodo de la red de sensores inalámbricos debe ser considerado del orden de los microamperios y vida útil mucho más prolongada. ¿Cómo lograr esto? Con la combinación de componentes de hardware de baja potencia en los equipos, sumado a técnicas de ciclos de trabajo muy cortos.

Durante la operación activa del nodo, la comunicación radial constituye la fase donde se produce el mayor consumo energético. Los algoritmos y protocolos se desarrollan de tal manera de reducir estos tiempos a un mínimo.

\subsubsection{Flexibilidad}

Cada escenario propondrá diferentes intercambios de cada uno de los parámetros de los equipos al considerar las posibilidades de trabajo de cada WSN. Con esto se obliga a que la arquitectura de los nodos sea flexible y adaptable a ellas. Para ello deben considerárselos siguientes términos: el ciclo de vida, tiempo de respuesta y procesamiento en la red. Por otro lado, cada uno de los nodos debe poseer solamente el hardware y software que demanda la aplicación en cuestión; caso contrario no sería todo lo económico que se pretende que sean. Por ello los nodos precisan de una gran modularidad de software y hardware, de tal manera que corriendo diferentes aplicaciones, no pierdan rendimiento ni eficiencia.

\subsubsection{Robustez}

Definimos robustez de un nodo sensor como la propiedad del mismo de soportar cambios en los procedimientos, aplicaciones o circunstancias en las que se utiliza. Para soportar el ciclo de vida requerido, cada nodo debe ser construido tan robusto como sea posible. 
En aplicaciones típicas de WSNs decenas o centenas de equipos deben trabajar armoniosamente por años. Para ello la red en su totalidad debe tolerar y adaptarse al caso de fallas individuales. Cada nodo, por tanto, debe ser diseñado en función de esta propiedad intrínseca de las WSNs. La modularidad del sistema es la herramienta más poderosa al momento de diseñar un sistema robusto.

La división de la funcionalidad del sistema total en subsistemas aislados, permite el control profundo de cada uno de los componentes previo al montaje final. Cada uno de los componentes debe ser lo más independiente posible, previendo interfaces definidas de manera de atender a interacciones inesperadas.

Como estas redes coexisten normalmente con otros sistemas inalámbricos, es indispensable la robustez de los vínculos inalámbricos. Con esta finalidad se suelen disponer de esquemas radiales multifrecuencias, ó a la utilización de spread-spectrum.

\subsubsection{Seguridad}

Los nodos de una WSN, como cualquiera de los equipos interconectados, son susceptibles a incidentes de seguridad. Esta susceptibilidad tiene que ver con la capacidad de los nodos de ser reprogramados. Explotando esta característica los desarrolladores actualizan el código ejecutable en solo nodo, replicándola aplicación en todo el universo de las WSN.

Para cumplir con los requisitos de seguridad establecidos en la WSN, cada uno de los nodos debe ser capaz de ejecutar rutinas complejas de autenticación y rutinas de encriptación de datos. La única manera de mantener la privacidad de la información en las redes de la WSN consiste en la encriptación de los datos debido a la susceptibilidad de intercepciones de las comunicaciones inalámbricas. Esta tarea debe ser 
realizada exclusivamente por la CPU, o con el auxilio de aceleradores criptográficos [105], [106]. Además de asegurar los datos transmitidos, los nodos deben asegurar también los datos almacenados por el nodo.

\subsubsection{Comunicación}

El esquema común de comunicación de los nodos es utilizar señales de radio frecuencia (RF) para comunicarse a través de distancias relativamente cortas. Con ello se minimiza el tamaño del equipo y su consumo energético.

Si bien hemos hecho notar anteriormente que la cobertura de la red no tiene que ver con el alcance de la transmisión de cada nodo, este parámetro influye en el momento que debamos definir el impacto de la densidad de nodos en la WSN. Si la distancia entre nodos es amplia, es probable que no podamos mantener la interconexión de la red, y si lo logramos, puede ser posible no poder asegurar suficiente redundancia para mantener el nivel aceptable de confiabilidad de la misma. La mayoría de los escenarios poseen una densidad natural de sensores que se corresponde con la granularidad de medición que deseamos. Si las comunicaciones de la red demandan una mayor densidad de nodos, simplemente se debe adicionar aquellos que se consideren necesarios por los objetivos del proyecto.

El alto rendimiento final de una conexión se corresponde con la capacidad de los nodos de lograr altas tasas de transferencia efectiva de datos con un consumo bajo de energía. Mientras la velocidad de transferencia aumenta, disminuye el tiempo de comunicación, por lo que el consumo disminuye consecuentemente. Sin embargo el aumento de velocidad de transferencia radial de la información se condice con un aumento de la energía consumida por el radio. 


\subsubsection{Capacidad de Cálculo}

Las operaciones más frecuentes en cualquier WSN son: 1) el procesamiento de los datos dentro de la red, y 2) el proceso de manejo de los protocolos de comunicación de bajo nivel.

Existen requerimientos de tiempo real asociados tanto con la comunicación como en el monitoreo. A medida que la información esta arribando al nodo, la CPU debe controlar la radio, decodificar el mensaje, almacenar en memoria los datos necesarios, etc. A medida que más rápida sea la tasa de transferencia de información en la WSN, mayor deberá ser la capacidad de cálculo del nodo.

Comúnmente el mayor tiempo de sus ciclos, las CPUs realizan procesamiento corriente de operaciones que incluyen: filtrado digital, cálculos de valores medios, detección de umbrales en mediciones, análisis de correlación y datos espectrales.

\subsubsection{Sincronización}

Teniendo en cuenta que la red de sensores inalámbricos debe realizar tareas específicas, las que normalmente deben correlacionarse con las mediciones de otros nodos, es preciso mantener una sincronización con los otros miembros de la red.

Los nodos de una WSN para poder comunicarse deben estar "despiertos", y cuando no se comunican deben permanecer "dormidos" para así ahorrar energía. Ambos estados deben corresponderse entre los estados de los nodos participantes de la comunicación. Los errores ó problemas en la sincronización conllevarán a que se realicen más ciclos de trabajo debido a ésta ineficiencia, degradando con ello la performance de la red. 
En los sistemas distribuidos se cumple que los relojes de los equipos van aumentando la diferencia existente entre ellos debido a mecanismos ineficientes de sincronización [107]. La única forma posible de sincronización entre los procesadores de cada nodo de la WSN es utilizando apropiadamente la red de comunicaciones.

Los mecanismos utilizados normalmente presentan variaciones de acuerdo a la temperatura, voltaje y humedad, por lo que el mecanismo de sincronización de los nodos debe ser un proceso preciso; tendiendo a compensar continuamente todos y cada uno de los factores que atenten contra la sincronización buscada.

\subsubsection{Tamaño y costo}

Los nodos de las WSN ese encuentran en el mercado en la forma de dispositivos que se convierten en parte de la red de comunicación. Estos nodos están lejos del ideario de tamaño comparable con el polvo (dustsized). Actualmente se encuentran dispositivos en tamaños desde monedas hasta de un paquete de naipes. Los precios que se deben abonar dependen de la capacidad de procesamiento, de la capacidad de procesamiento, de los requerimientos de instalación; y como en cualquier otra transacción comercial, del número de equipos solicitado.

El tamaño y costo de cada uno de los nodos afectará a otros parámetros a considerarse en la instalación de la WSN. Con una fácil instalación, se disminuyen los costos de implementación. Estos dos factores son los decisivos cuando se debe tomar una decisión sobre la adopción la tecnología para un determinado proyecto, cuando existen varias alternativas tecnológicamente viables. Los nodos inalámbricos operados por baterías no precisan de cables para su instalación, requiriéndose solamente un destornillador común. 
Los primeros usuarios de estas tecnologías han debido abonar por los nodos de sus WSN unos pocos cientos de dólares. En nuestros días es posible obtener precios mucho más convenientes. Un reporte de Intel indica que: “... con reingeniería, la ley de Moore y grandes volúmenes de producción, el precio de los nodos podría estar debajo de los cinco dólares en los próximos años" [108].

Las redes WSN proyectan normalmente sobre la existencia de un presupuesto fijo, siendo el objetivo principal la toma de muestras en la mayor cantidad de lugares posible, dentro del límite del presupuesto. Reduciendo el costo de cada equipo, se pueden aumentar el número de nodos, y con ello la densidad de equipos.

No se debe olvidar que el tamaño facilita la instalación de los equipos en el escenario elegido, aumentando con ello el coeficiente de portabilidad del equipo.

\subsection{Prestaciones del Hardware}

Consideraremos aquí las diferentes variables a tener en cuenta al considerar cada uno de los elementos que componen un nodo en las WSN. La prestaciones que debemos considerar adecuadas en cada proyecto son la tasa de transferencia, el consumo energético, la memoria, y los costos.

\subsubsection{Energía}

La limitación de disposición de energía en los nodos individuales es el mayor inconveniente a superar cuando se considera el diseño de los equipos a instalar en una WSN. Para obtener largas autonomías debe considerarse un bajo consumo. Existen algunos preceptos que se deben respetar siempre que se quiera optimizar el consumo energético en los 
nodos. Una consideración fundamental es disminuir la potencia irradiada utilizando redes ad hoc y métodos de comunicación multisalto; lograr un balance óptimo entre los procesos de comunicación y de cálculo; diseñar de sistemas de radio mucho más eficientes, que cumplan los requerimientos de las WSN; y por último desarrollar protocolos de ruteo y algoritmos más eficientes considerando las características de las WSN.

Las WSNs han sido destinatarias de numerosos trabajos en lo académico e industrial, fundamentalmente por el amplio rango aplicaciones para las cuales se las considera apropiadas [109]. Los trabajos de investigación sobre WSNs se han enfocado, desde sus orígenes, con la premisa que los nodos necesariamente utilizan una fuente portátil y limitada de energía. Este es el insumo principal por lo que la mayoría de los trabajos han apuntado al desarrollo de protocolos que maximicen la eficiencia energética, mejorando la vida útil de las mismas [110].

Los ciclos de operación de un nodo normalmente son del tipo: dormirdespertar-sensar-comunicar. El mayor tiempo del ciclo se desarrolla en la etapa de bajo consumo, es decir durante dormir [111].

Existen escenarios donde la vida útil esperada de una WSN sea más prolongada que en otros, proyectando para este valor muchos años; incluso décadas. Considérense los cases de los proyectos de monitoreo ambiental ó de SHM donde el reemplazo de las baterías es una tarea muy difícil, cuando no imposible.

Para el diseño y operación de todos los aspectos de las WSN se requiere el uso de una metodología de diseño bien estructurada, comenzando por la subyacente plataforma del hardware, el software y los protocolos de red con el objetivo final de maximizar la vida útil.

Teóricamente, una batería de 1000 mAh podría mantener un equipo consumiendo $10 \mathrm{~mA}$ durante 100 horas. Sabido es que esta regla no es 
aplicable como se expresa. A medida que las baterías consumen la carga que poseen, la diferencia de tensión entre bornes disminuye. Esto se debe a los diferentes procesos químicos que tienen lugar en la batería, por lo que el voltaje varía contantemente. Si el sistema no fuera tolerante a la variación de voltaje, sería imposible utilizar la totalidad de la carga almacenada en la pila para realizar trabajo útil en la WSN. Una batería alcalina de $1,5 \mathrm{~V}$ no es considerada por sus fabricantes como exhausta hasta que la misma alcance una lectura de 0,9 V [112].

\subsubsection{Tecnología de las baterías}

Existe un gran número de baterías en el mercado, cada una de las cuales posee ventajas y desventajas cuando deben utilizarse como alimentación de modernos equipos electrónicos. Los tipos más frecuentes de baterías utilizadas en WSNs son tres: alcalinas, ión-litio, y de hidruro de Níquel.

Una batería alcalina AA está normalizada a 1,5 volts, pero durante la operación la tensión entre bornes alcanza valores desde 1,65 a 0,8 Voltios. La máxima intensidad de corriente de las baterías alcalinas alcanza un máximo a $2.850 \mathrm{mAh}$. Estos valores pueden apreciarse en la ilustración 3.9. Considerando el volumen aproximado de esta batería (8,5 $\mathrm{cm}^{3}$ ), posee aproximadamente una densidad energética de 1.500 Joule/cm ${ }^{3}$.

Si bien la batería alcalina es la más económica, y posee una alta capacidad, la principal desventaja la presenta en el amplio rango de los voltajes entregados a los equipos conectados.

Las baterías de ión-litio no recargables son una excelente fuente de energía compacta. La más pequeña es solamente es unos pocos milímetros de diámetro. Su ventaja respecto a las baterías alcalinas se basa en el hecho de proporcionar una tensión contante, ó que varía muy 
poco, a medida que se descarga (ver la ilustración 3.10). Los equipos que funcionan con estas baterías no deben ser tan tolerantes a los vaivenes en cuanto a niveles de voltajes provistos por las baterías, como es el caso cuando se hace uso de las baterías alcalinas.

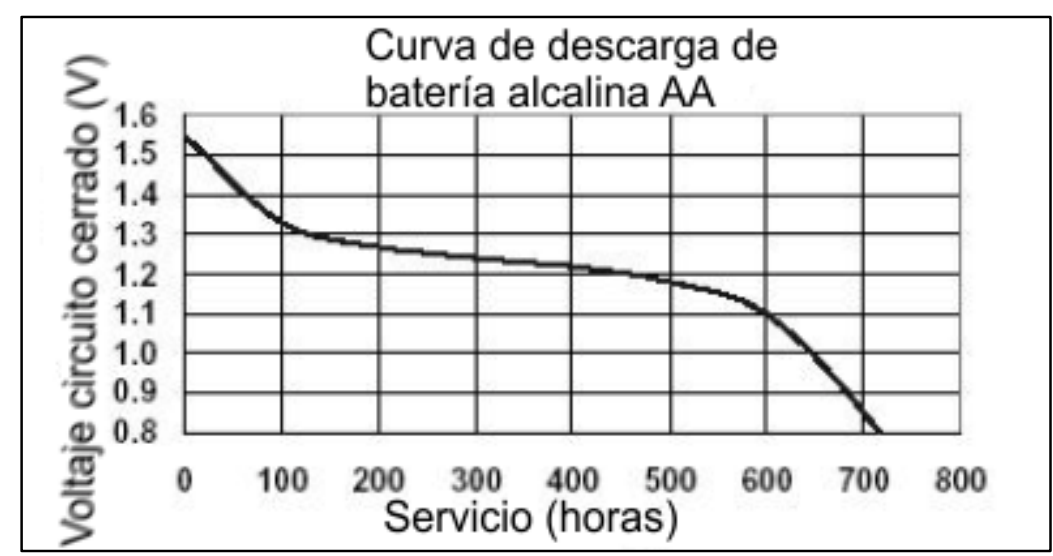

llustración 3.9. Curva de descarga de Batería alcalina AA

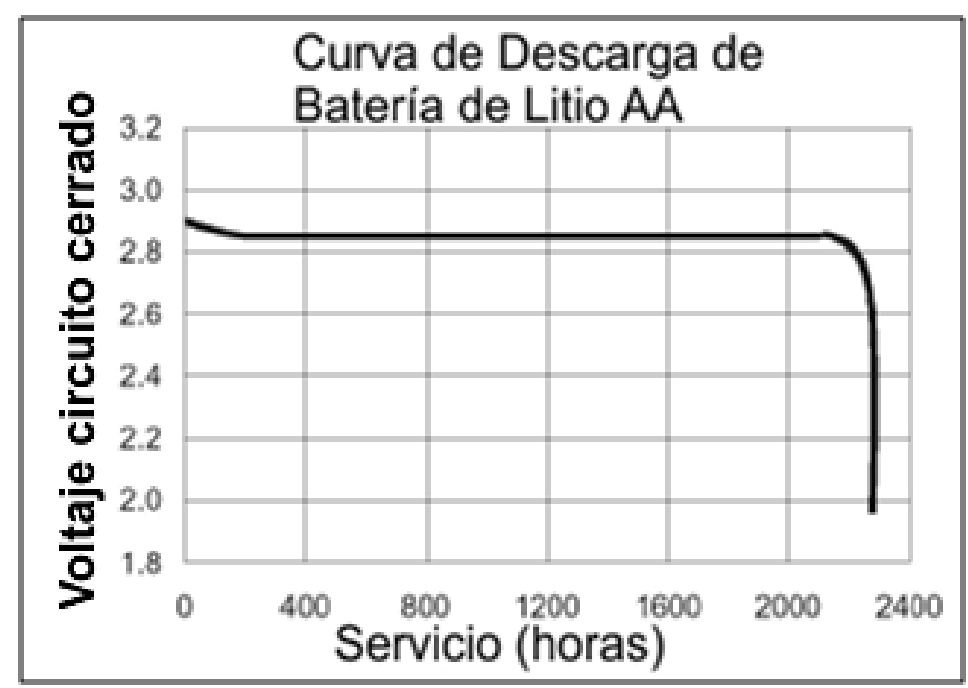

Ilustración 3.10. Curva de descarga de batería de Litio

Las baterías de hidruro de Níquel poseen la ventaja de ser fácilmente recargables, y como desventaja principal a su baja densidad energética. 
Una batería de hidruro posee la mitad de densidad energética de una batería alcalina, costando la batería de $\mathrm{NiMH} 5$ veces más.

\subsubsection{Regulación de Voltaje}

Mientras la velocidad del procesador y la capacidad de la memoria han exhibido un crecimiento de varios órdenes de magnitud en los últimos años, la densidad energética de las baterías solamente se ha triplicado, quedando claro que el almacenamiento y provisión energética no ha evolucionado en la misma manera que la tecnología en las WSN [113]. Ante el escenario cierto de la imposibilidad de recarga ó reemplazo de las baterías en campo, la energía deviene en el recurso más preciado de las WSN.

Para reducir los efectos indeseados de la variación del voltaje a los nodos sensores, es norma utilizar técnicas de regulación, que normalmente requieren que el voltaje de entrada sea mayor que el voltaje de salida.

La mayor eficiencia que se obtiene de los reguladores de voltaje alcanza al $90 \%$, en sistemas donde el consumo alcanza a cientos de miliamperios. Este valor de la eficiencia cae bruscamente cuando el consumo es menor al indicado.

El consumo energético propio de los reguladores es un costo muy alto que los sensores se ven obligados a pagar, ya que se producen en todo momento, aún cuando el equipo no esté utilizando energía para realizar sus actividades estándar.

\subsubsection{Energías renovables}

Una alternativa a las baterías descartables en los nodos de las WSN se encuentra en las energías renovables. El obtener energía de una fuente alternativa, como el proceso de almacenamiento de la misma, es una 
necesidad en muchas de las aplicaciones emergentes de las WSN, los implantes biomédicos, etc. Sabido es que éste proceso puede proveer la energía necesaria a lo largo de la vida útil de la WSN., por ello el diseñar un sistema que aproveche este potencial, requiere un profundo conocimiento de diversas y complejas interrelaciones en la red [114].

Este proceso no es ninguna novedad en cuanto a lo innovación tecnológica, pero lo que sí es nuevo es utilizar las ventajas de la comodidad de almacenamiento alternativo en dispositivos modernos como los nodos sensores; cuando estos presentan serios inconvenientes en lo que a sus limitaciones físicas se refiere. Considere que los molinos de viento y los generadores hidroeléctricos han estado en funcionamiento desde hace muchos años. Estos ejemplos han tratado de traducirse en aplicaciones en WSN a través de miniaturizaciones para poder ser utilizados en sistemas integrados. Las trabajos prácticos realizados han conllevado a eficiencias muy bajas.

La diferencia sustancial desde el punto de vista del manejo energético que existe entre los sistemas alimentados por baterías y aquellos que son parte de sistemas de aprovechamiento de energía alternativa, es que las métricas de optimización energéticas convencionales no podrían ser utilizadas en los escenarios donde se realice algún tipo de aprovechamiento.

Frente a este escenario, un objetivo de diseño podría ser que el nodo opere el mayor tiempo posible en modo neutral, lo que se corresponde con utilizar la energía que se corresponde con aquella cantidad que es almacenada. En esta condición de diseño, podríamos alcanzar una vida útil indefinida, limitada solamente por la longevidad de los equipos utilizados.

El avance del aprovechamiento de las energías alternativas se ha correspondido con un avance importante en el desarrollo de tecnologías 
electrónicas de bajo consumo y potencia [115]. Los paneles fotovoltaicos con el paso de los años son más económicos y mucho más eficientes. Teniendo en cuenta los innumerables proyectos existentes sobre utilización de energía solar en el planeta, ha quedado demostrado que el sol es una fuente confiable de energía.

La energía capaz de ser almacenada ded cada una de las fuentes alternativas es muy variable, y generalmente escasa; requiriendo el desarrollo de arquitecturas y circuitos de bajo consumo energético que deben escalar con los recursos existentes del aprovechamiento realizado.

Los dispositivos de almacenamiento energético no son dispositivos que proveen energía continuamente. Los modelos de disponibilidad energética son utilizados para establecer la utilización eficiente de los recursos en el nodo.

Las celdas solares modernas son capaces de generar hasta $10 \mathrm{~mW}$ por pulgada cuadrada si son colocadas directamente al sol. Si esta energía es almacenada adecuadamente, puede ser utilizada en horarios sin presencia solar.

Normalmente las baterías recargables son utilizadas en forma conjunta con la energía solar. Las baterías son recargadas directamente durante las horas del día con suficiente luz solar. En la ilustración 3.11 se muestra el panel fotovoltaico, una batería, el módulo controlador de energía y la caja estanca. 


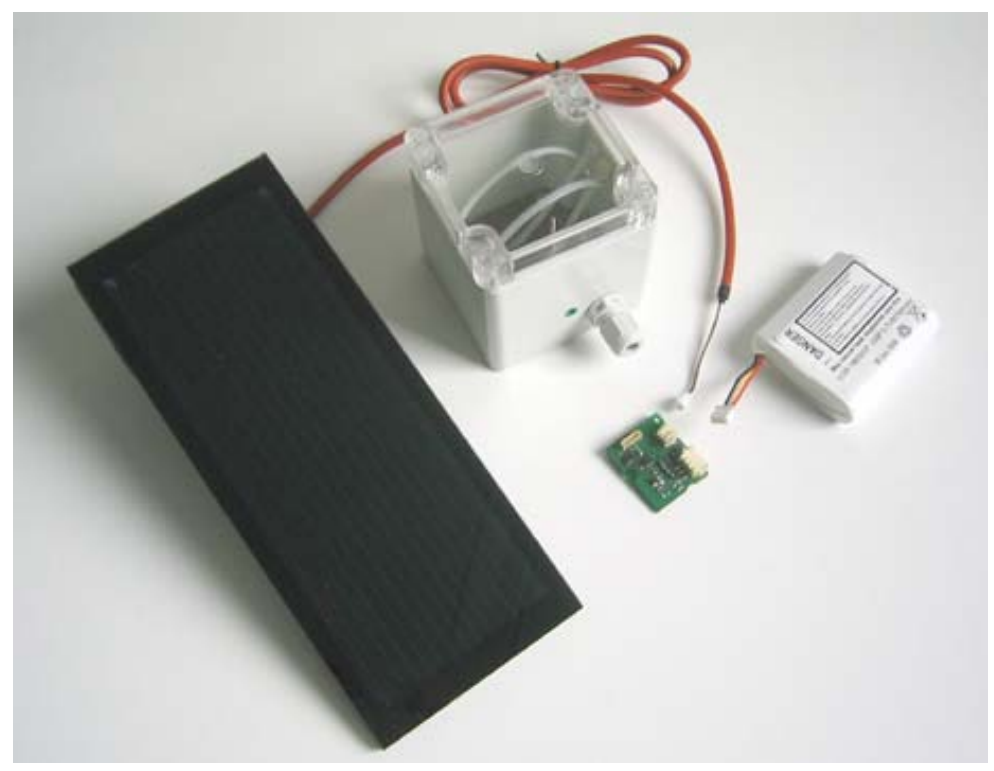

llustración 3.11 Módulo generación-almacenamiento de energía en nodos de WSN

(a)

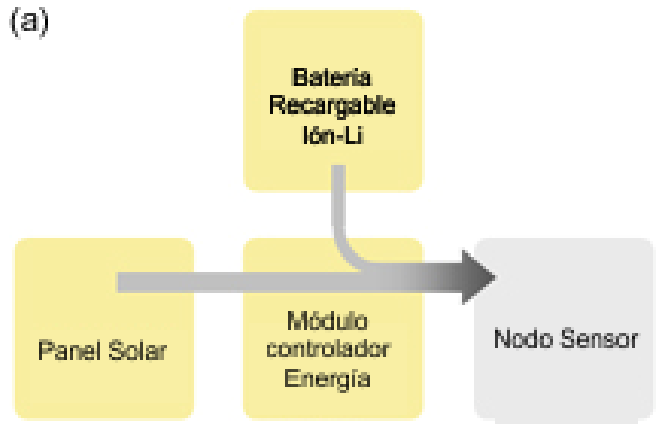

(b)

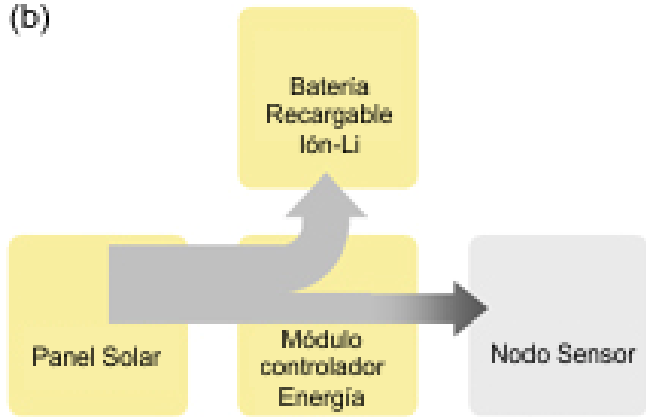

\section{Ilustración 3.12. Funcionamiento del controlador energético de un nodo de WSN [16]}

El módulo controlador de energía distribuye la energía captada por el panel solar en una forma inteligente. En el caso que la generación momentánea de energía no sea suficiente para alimentar al nodo, se complementa el consumo con energía almacenada en la batería, como se observa en la llustración 3.12 (a) [16]. Caso contrario, el excedente 
generado es almacenado en la batería para su utilización en momentos oportunos.

\subsubsection{Radio}

En las WSN, el subsistema de radio es el más importante, debido a que se trata del mayor demandante de energía del sistema. Por ello los desafíos más importantes al diseñar tecnologías de radio para las WSN distribuidas, apuntan al consumo de las mismas y al tamaño del sistema radial.

El consumo energético del sistema de radio es la limitante en la implementación y desarrollo de WSN de muy bajo consumo, siendo la línea de trabajo preferida de investigadores [116]. Hemos visto que la capacidad de las baterías normalmente no es suficiente para mantener a los equipos en funcionamiento continuo a lo largo de una vida útil aceptable, por lo que se deben diseñar adecuadamente los ciclos de trabajo. Por supuesto esto representa un impacto en la forma de proyectar y desarrollar sistemas de radio para las WSN.

Para lograr un tamaño cada vez más pequeño de nodo, se requiere un alto grado de integración de todos los componentes, de manera que pueda caber completamente en un circuito integrado. Con esta orientación se han desarrollado los system-on-chip, que en general son del tipo aplicado en las WSN. En transceptores modernos, el consumo de los equipos para transmisiones a corta distancia; el consumo promedio es entre 15 y 300 milliwatts, durante los modos de transmisión y recepción.

Una idea equivocada en las redes de datos inalámbricas es aquella que asume que la recepción no importa costos energéticos. La mayor parte de la energía utilizada por la radio es utilizada para la operación interna de la misma; y solamente una baja proporción de la misma es emitida por la 
antena, por lo que el consumo mayor de los nodos puede ser dominado por el consumo en modo recepción.

Las primeras redes inalámbricas de sensores estaban conformadas por equipos que poco hacían con respecto al ahorro energético. Esto ha cambiado notablemente con los equipos de segunda y tercera generación hoy en el mercado. Se ha reemplazado el antiguo hardware propietario con sistemas de radio estandarizados, de ancho de banda hasta 250 kilobits por segundo, los que son administrados por controladores mucho más modernos y poderosos.

La ilustración 3.13 nos ayuda a realizar una revisión sobre las tecnologías inalámbricas utilizadas actualmente, y su relación en cuanto a ancho de banda y consumo energético.

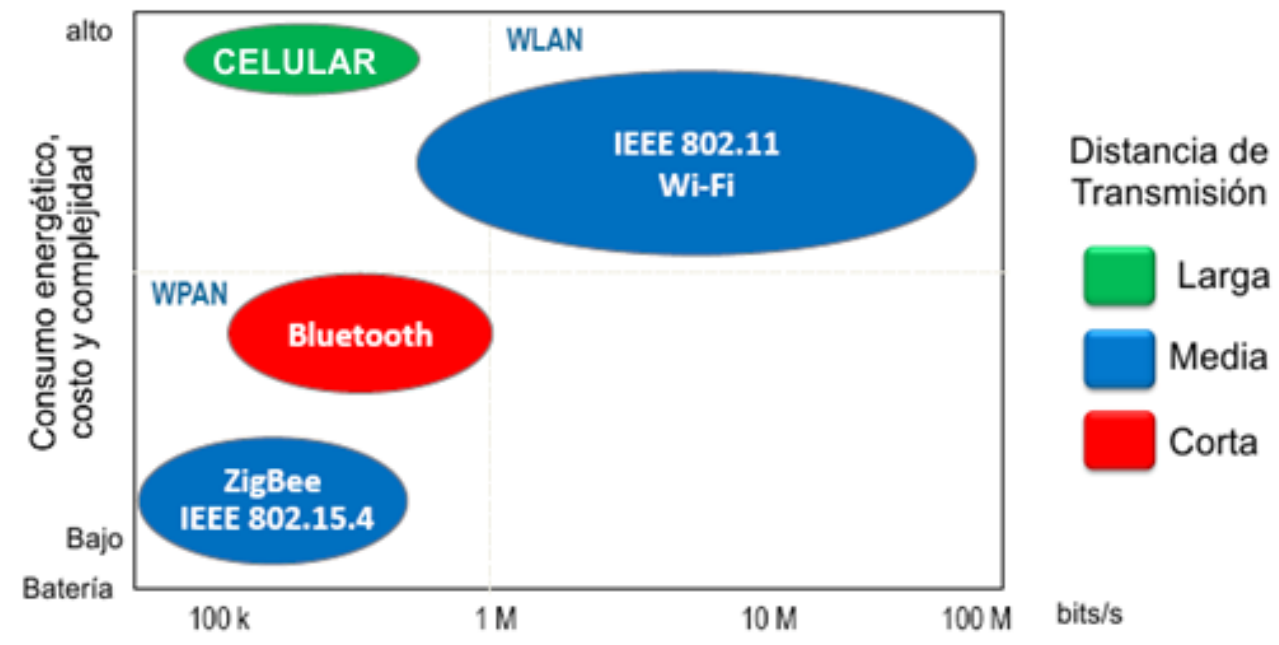

Ilustración 3.13. Estándares de comunicaciones inalámbricas y sus consumos

Respecto al espectro electromagnético disponible para la emisión radial, la mayoría de los nodos de las WSN son libres de modular en el rango de las frecuencias industriales, científicas y médicas (ISM), siendo esta una 
ventaja comparativa para la tecnología. En la Tabla 3.3 se indican las frecuencias ISM [117] disponibles para aplicaciones en la industria inalámbrica.

Tabla 3.3 Banda de frecuencias disponibles de ISM para aplicaciones

\begin{tabular}{|c|c|}
\hline Banda de frecuencias & Frecuencia central \\
\hline $6.765-6.795 \mathrm{MHz}$ & $26.957-27.283 \mathrm{MHz}$ \\
\hline $13.553-13.567 \mathrm{MHz}$ & $13.567 \mathrm{MHz}$ \\
\hline $26.957-27.283 \mathrm{MHz}$ & $27.12 \mathrm{MHz}$ \\
\hline $40.66-40.70 \mathrm{MHz}$ & $40.68 \mathrm{MHz}$ \\
\hline $433.05-434.79 \mathrm{MHz}$ & $433.92 \mathrm{MHz}$ \\
\hline $902-928 \mathrm{MHz}$ & $915 \mathrm{MHz}$ \\
\hline $2,400-2,500 \mathrm{MHz}$ & $2,450 \mathrm{MHz}$ \\
\hline $5.725-5.825 \mathrm{GHz}$ & $5.8 \mathrm{GHz}$ \\
\hline $24.00-24.25 \mathrm{GHz}$ & $24.125 \mathrm{GHz}$ \\
\hline $61.00-61.50 \mathrm{GHz}$ & $61.25 \mathrm{GHz}$ \\
\hline $122-123 \mathrm{GHz}$ & $122.5 \mathrm{GHz}$ \\
\hline $244-246 \mathrm{GHz}$ & $245 \mathrm{GHz}$ \\
\hline
\end{tabular}

\subsubsection{Alcance de transmisión}

El alcance de la transmisión de los nodos en una red de sensores inalámbricos depende de numerosos factores. El más intuitivo de todos ellos es la potencia de transmisión; cuando más potencia posea la señal, más lejos alcanzará a propagarse. La relación entre potencia de la señal y distancia alcanzada es una ecuación polinomial de grado entre 3 y 4 para la propagación fuera de la línea de visión [118], por lo que para transmitir a una distancia dos veces mayor se necesitará aumentar la energía de transmisión entre 8 a 16 veces.

Una alternativa que mejora tanto lo referente a consumo energético, como a la calidad de los enlaces es lograr la disminución de la potencia de transmisión utilizando la comunicación multisalto. Con ello acortamos las 
distancias de los vínculos entre los nodos, a la vez que aumentamos la densidad de nodos mejorando la conectividad.

Normalmente en las instalaciones de WSN no es posible utilizar antenas direccionales de alta ganancia, sencillamente porque no se efectúa alineación alguna de los nodos. Los nodos son provistos, en la mayoría de los casos, con una antena omnidireccional, permitiendo establecer vínculos en todas direcciones.

\subsubsection{Modulación y Tasa de Transferencia}

Diferenciándose de las redes inalámbricas a las que nos hemos acostumbrado al uso diario, en las WSN la tasa de transferencia no es un valor elevado. Este valor, para las mayorías de las aplicaciones, trepa hasta un valor entre 10 y 100 kilobits por segundo. A medida que la tasa de transferencia aumenta, disminuye el tiempo de transmisión. Siendo la fase de transmisión la de mayor consumo energético instantáneo, es esencial que la radio permanezca inactiva el mayor tiempo posible.

\subsubsection{Tiempo de encendido y "power-aware"}

El modo en el cual la radio opera para conmutar entre sus estados de operación "dormido" y "activo", es un parámetro importante para dimensionar y calcular la eficiencia global de la WSN. Normalmente en todos los estudios se asume que el equipo estará encendido solamente cuando es necesario realizar alguna tarea.

En una WSN, realizar las mediciones y enviar los datos pueden ser tareas a realizar, sin necesidad de establecer una organización temporal de las mismas. Estas dos tareas hemos visto anteriormente que se realizan sin posibilidad de ahorro energético, sin considerar el procesamiento y la compresión de datos. La cuestión que debe plantearse es: ¿cuándo 
deben pasar los nodos al estado "dormido" y cuando deben estar "despiertos"? Esta respuesta estará dada por la interacción propia del equipo con el mundo real.

Un sensor del tipo "power-aware" administra el consumo energético a lo largo del ciclo de trabajo a través de diferentes estados de "dormido". Por supuesto cada uno de los componentes de los nodos posen sus diferentes estados de consumo energéticos, por ejemplo el procesador, puede estar activo, dormido ó inactivo; mientras que los modos de radio normalmente en un nodo WSN puede ser uno de los siguientes: transmisión, recepción, espera o desconectado. Cada uno de estos estados, por ejemplo del MCU se diferencia por los diferentes consumos en los diversos intervalos tales como el habilitar la funcionalidad, overead de cambio de modo y el tiempo requerido para "despertarse" desde una función de bajo consumo [119].

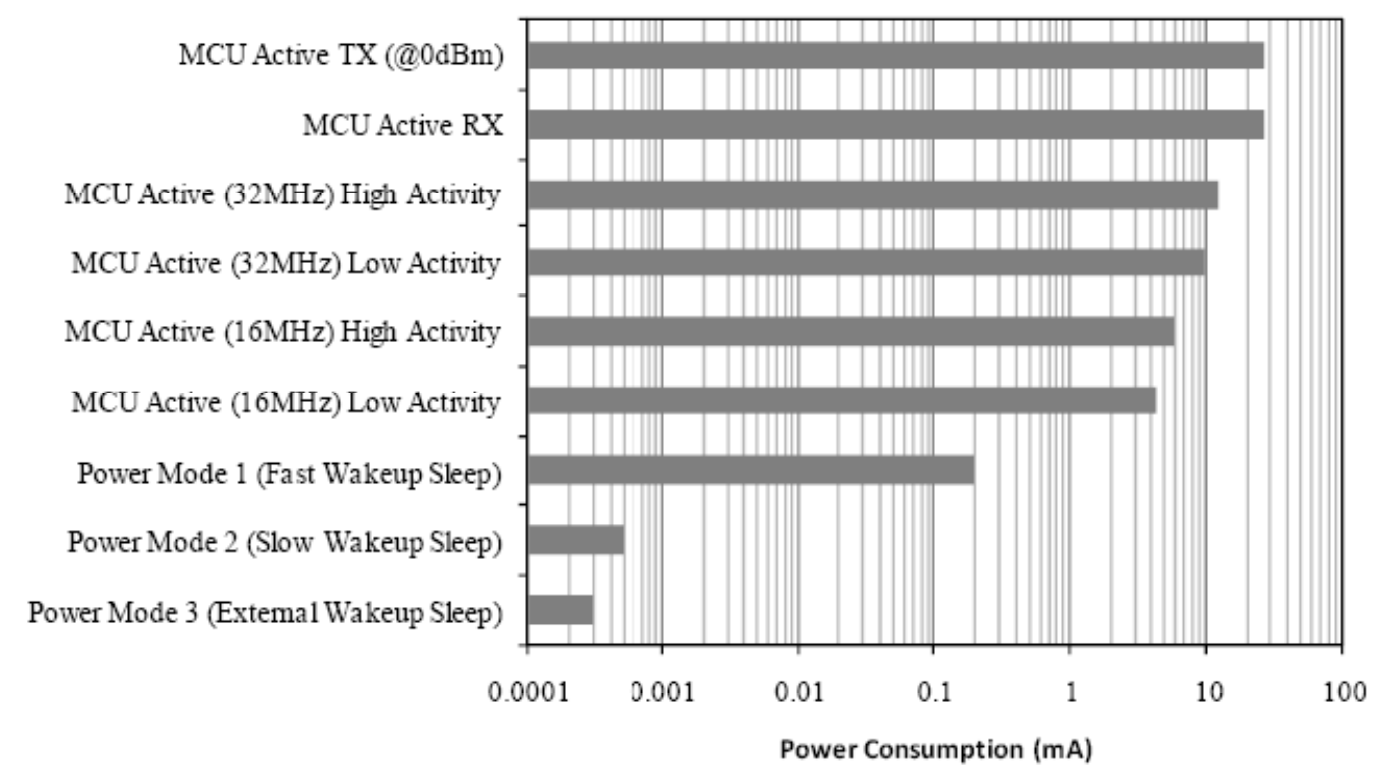

Ilustración 3.14 Consumo energético del circuito TI CC2430 en diferentes modos 
En la ilustración 3.14 se muestran los diferentes valores de consumo energético para un sistema integrado (SoC) de un circuito Texas Instruments CC2430 [120], donde se observan que los mayores consumos se producen en los modos activos.

\subsection{Controladores}

La unidad de procesamiento (CPU ó $\mathrm{MCU}^{1}$ ) es el corazón de cualquier computador. En un nodo de WSN, la CPU recoge los datos originados en los sensores; los procesa, decide cuando y donde enviarlos, recibe datos de otros sensores y establece el comportamiento del actuador. Generalmente se encarga de correr varios programas, algunos de ellos simultáneamente; siendo los mismos consistentes en procesamiento de señales y protocolos de comunicación.

Las características de las MCU son en general, manejo sencillo de datos, número reducido de instrucciones y diversos modos de ahorro energético, para así obtener una buena relación entre ahorro y reacción ante eventos (tiempo de wakeup).

Estas tareas pueden llevarse a cabo por varias arquitecturas de controladores, representando cada una de ellas una relación distinta de flexibilidad, performance, eficiencia energética y costos. Una de las alternativas es la utilización de procesadores de propósito general, como aquellos que son utilizados en las PC.

Los procesadores en las computadoras personales están sobredimensionados en cuanto a potencia de cálculo, y el consumo de energía de los mismos es excesivo. Como una alternativa a las CPU de PCs existen procesadores más simples, los que han sido desarrollados

\footnotetext{
${ }^{1}$ Micro Controller Unit
} 
específicamente para trabajar en forma conjunta con sistemas integrados. Debido al aumento de la demanda de capacidad de cálculo a los nodos de WSN, los procesadores de 16 y 32 bit se han vuelto normales al momento de seleccionar MCU para sinks, gateways y nodos de acceso a internet. En la última generación de hardware de sensores, se ha normalizado la utilización de sistemas operativos integrados [121] tales como TinyOS, Jennic BS, y otros.

Una de las cualidades de estos microcontroladores es su flexibilidad de conexión con otros dispositivos, como por ejemplo con sensores. Además otra de sus características es que son fácilmente programables en lenguajes abiertos, siendo por lo tanto sumamente flexibles. Estos procesadores no están diseñados específicamente para ser utilizados en nodos que se caracterizan por ser orientados a eventos, sino que la mayoría de los MCU se basan en el presupuesto que la máquina será utilizada para la computación monolítica de propósito general.

Otra alternativa a ser utilizada en los controladores de WSN es partir desde la alta flexibilidad ofrecida por un microcontrolador y la utilización de Field-Programmable Gate Arrays (FPGAs) [122], [123], ó como otra alternativa, Application-Specific Integrated Circuits (ASICs) [124]. En el caso de las aplicaciones dedicadas para WSN, en las cuales las tareas de los nodos sensores no cambian a lo largo de su vida útil, y el número de nodos es suficientemente grande de manera de asegurar la inversión en un desarrollo ASIC, este podría ser la mejor solución. Sin embargo al estar de la tecnología de WSN hoy día, la mayor flexibilidad y la simpleza en la utilización de microcontroladores hace de éstos la solución preferida. 


\subsubsection{Microcontroladores}

Existen diferentes productos tanto entre microcontroladores, como aquellos que presentan soluciones completas para los ambientes de redes de sensores.

Los microcontroladores utilizados en varios prototipos de sensores incluyen el procesador Atmel ó como indicáramos anteriormente el MSP 430 de Texas Instruments. En prototipos más antiguos han sido utilizados también los procesadores StrongArm de Intel, pero no es considerado como hoy como una alternativa válida. Sin embargo, dado que las propiedades principales de cada uno de estos procesadores y controladores es bastante similar, las conclusiones de estos trabajos pioneros son aún válidas.

- Intel StrongARM: considerandos el ambiente de las WSN, éste procesador es considerado de alta gama, dado que está orientado a ser utilizado en dispositivos portátiles como PDAs. El Modelo SA1110 [125] posee un core RISC, con una velocidad hasta $206 \mathrm{MHz}$.

- Texas Instruments MSP 430: TI provee una familia completa de microcontroladores bajo esta designación [126]. A diferencia del StrongArm, el MSP 430ha sido desarrollado para ser utilizado en sistemas embebidos. Posee un procesador RISC de 16 bits, una cantidad variable de memoria RAM en el chip (2 a $10 \mathrm{~KB}$ ) y varios convertidores analógicos/digital de 12 bits, como también un reloj de tiempo real.

- AVIDirector: Este producto de la firma estadounidense Avi Wireless utiliza Java en sus dispositivos M2M. Estos pueden conectarse a cualquier tipo de máquina, sensor o dispositivo. Son capaces de establecer vínculos con una gran cantidad de equipos utilizando enlaces inalámbricos, ya sean satelitales, Wi-Fi, 
Bluetooth o ZigBee. (ilustración 3.15). El sistema muestra una arquitectura abierta por lo que el usuario final puede construir su propio hardware y utilizar diferentes plataformas de software.

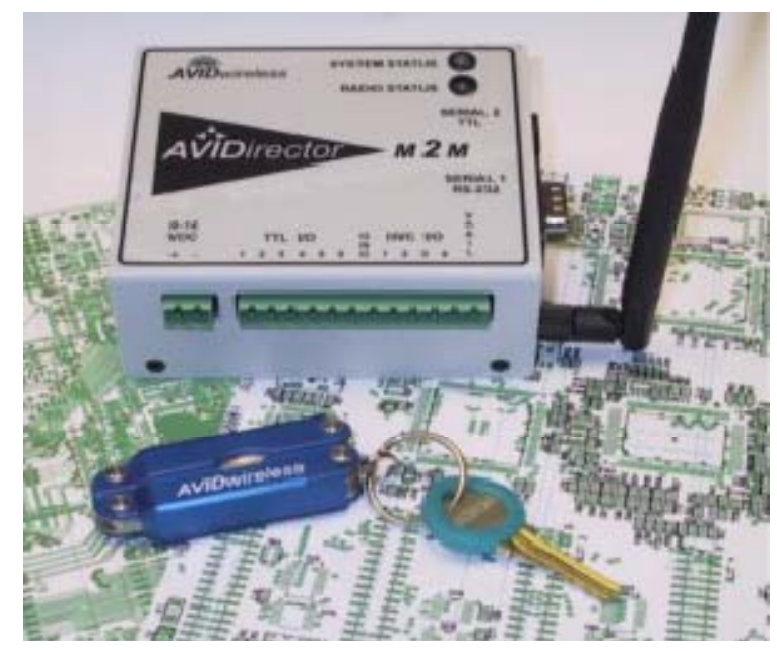

llustración 3.15. AVIDirector

- WMSN.P: El principal producto para WSN de la empresa Convergix (Bélgica) es el WMSN.P - 2812/D2 (ilustración 3.16), utilizando la banda ISM de 2,4 GHz. Una característica de éste nodo es su bajo costo, permitiendo la instalación de redes bastante densas.

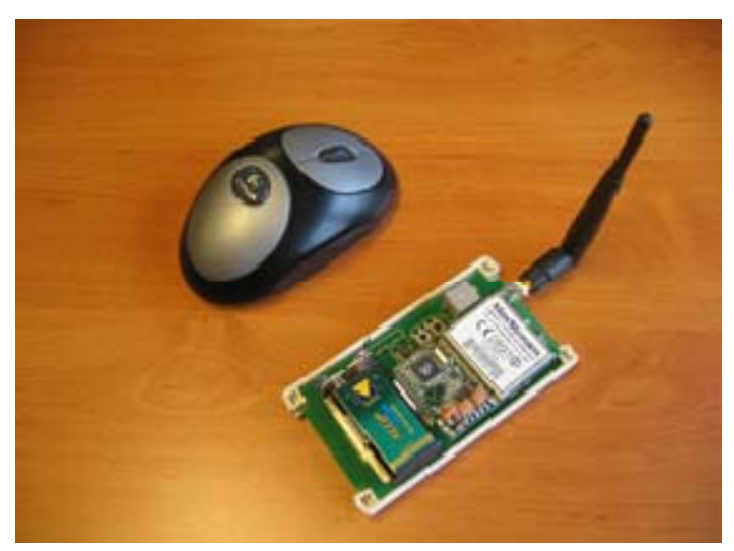

Ilustración 3.16. Dispositivo WMSN.P 
- JN5139/JN5148: El módulo Jennic JN5139 es compatibles con los microcontroladores de bajo costo y consumo IEEE802.15.4. El núcleo soporta un procesador RISC de 32 bits, transceptor compatible IEEE802.15.4 de 2,4 GHz, 192 KB ROM incluyendo el stack de protocolos y 96 KB RAM.

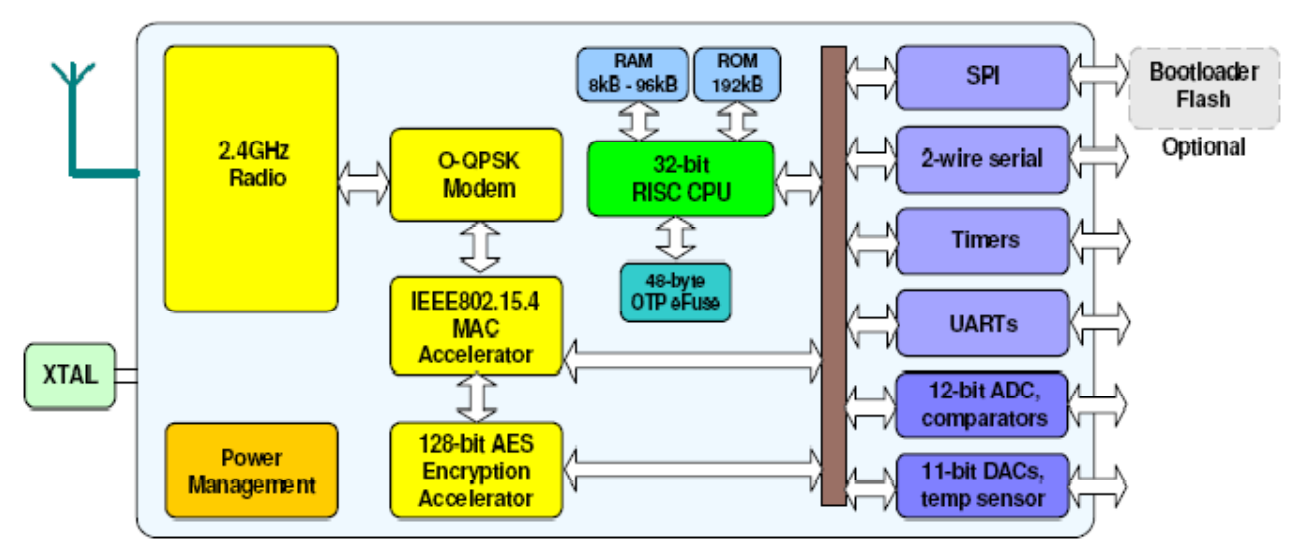

Ilustración 3.17. Plataforma Jennic JN5139

La plataforma JN5139 mostrada en la ilustración 3.17 [127] utiliza Acelerador MAC por hardware, al igual que el acelerador de encriptación AES de manera de reducir el consumo de energía y los ciclos de procesamiento. Posee un regulador de tensión pudiendo trabajar entre voltajes de batería de 2,2 y $3,2 \mathrm{~V}$.

- MeshScape La empresa Milennial Net ofrece a sus usuarios una variada cantidad de productos y servicios, con el objeto de desarrollar, mantener y utilizar las redes de sensores inalámbricos formadas por nodos MeshScape 4.0 (ilustración 3.18). 


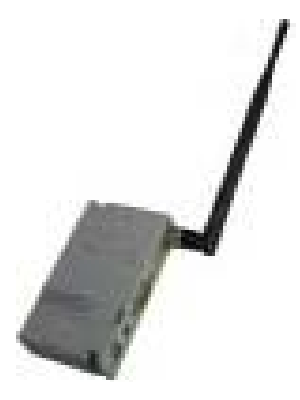

(a)

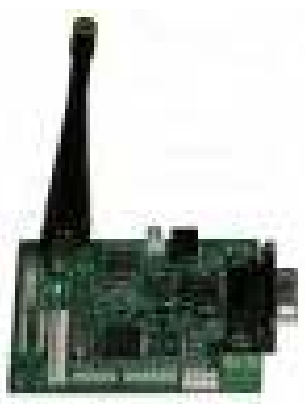

(b)

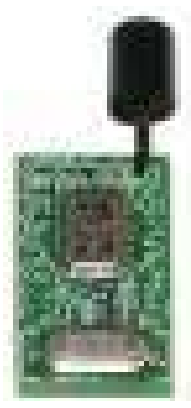

(c)

Ilustración 3.18. Tecnología de MeshScape. a) Módulo MeshGate b) Placa MeshNode c) Placa EdnBoard

Esta tecnología provee alta escalabilidad, confiabilidad, sensibilidad y eficiencia energética. Se encuentra disponible en las bandas de frecuencia de $916 \mathrm{MHz}$ y $2,4 \mathrm{GHz}$. Estos sistemas están formados por módulos de control como el MeshGate, Unidades de expansión como en MeshNodes y unidades sensores como EndNodes.

- SensiNet: Estos equipos son ofrecidos por la empresa SensiNet en dos variantes: el $\mathrm{H} 900(900 \mathrm{MHz})$ y el A2400 $(2,4 \mathrm{GHz})$. La redes SensiNet están formadas por Smart Nodes, Mesh Nodes, gateways y bridges. El Smart Node adquiere la información para el Mesh Node el cual encamina la información hasta alcanzar a los bridges o gateways. Ese Gateway conecta a la red con otras redes cableadas como Internet. El equipo SensiNet EMS Smart de la Ilustración 3.19, realiza monitoreo en tiempo real de temperatura y humedad. 


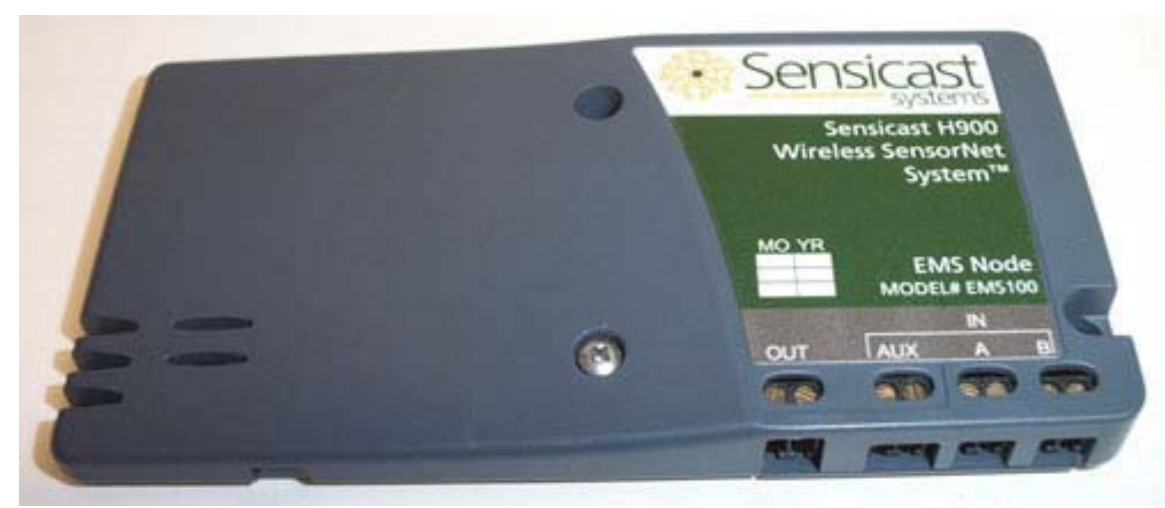

Ilustración 3.19. Nodo SensiNet EMS Smart

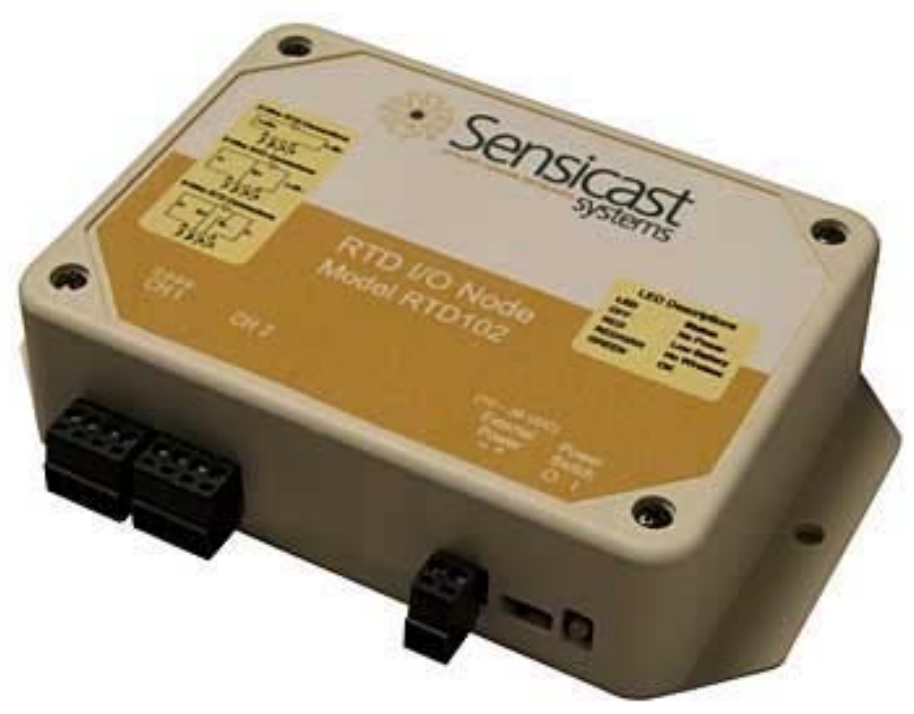

Ilustración 3.20. Nodo de la familia de sensores Sensinet RTD

La familia de sensores Sensinet RTD (ilustración 3.20) son apropiados para uso externo, soportando condiciones meteorológicas extremas. Al igual que la familia EMS, son aptos para medición de temperatura y humedad, pero no poseen ningún tipo de conexión externa. 


\subsection{Memoria}

El componente de memoria es bastante sencillo. Existe una necesidad de acceder a memoria de acceso aleatorio (RAM) para almacenar las lecturas intermedias del sensor, los paquetes de otros nodos, y otras tareas.

Mientras que la memoria RAM es rápida, su principal desventaja es que pierde su contenido si se produce un apagón. Por ello, se suele almacenar en la memoria ROM el código de programa, ó, más frecuentemente; en memoria EEPROM, o en memoria flash. La memoria flash puede servir como memoria intermedia para el caso que la RAM no sea suficiente, o en el caso que la energía deba ser interrumpida y la RAM se borre. Deben considerarse largos retrasos de acceso a lectura y escritura en la memoria flash, como también la energía que ello demanda.

\subsection{Equipo de Comunicaciones}

\subsubsection{Elección del medio de Comunicación}

El medio de comunicación es aquel que se utiliza para intercambiar información con los nodos de la red, en nuestro trabajo es excluyente la utilización de tecnologías inalámbricas. En algunos casos, cuando la práctica lo aconseje, las comunicaciones por medios cableados pueden ser el método apropiado. Los sistemas de comunicación para estos casos son generalmente soluciones enlatadas.

En el caso de comunicaciones inalámbricas, la primera decisión a tomar es la tecnología de transmisión: radiofrecuencia, óptica, ultrasonido u otro. De estas opciones, la comunicación basada en radiofrecuencias (RF) es por lejos la más relevante, y la que cumple con los requisitos de la mayoría de las WSN. Proveen vínculos de distancias considerables, tasas 
de transferencia y de error aceptables, consumo energético razonable y no requieren en ciertos casos de línea de visión entre emisor y receptor.

\subsubsection{Transceptores}

En la actualidad en cada nodo sensor es necesario un transmisor y un receptor. La tarea esencial de estos dispositivos es tomar la cadena de bits proveniente del microcontrolador y convertirlas en ondas radiales. Por razones prácticas se busca un equipo que cumpla ambas funciones en una sola entidad física. Normalmente la operación de estos equipos es de la forma half-duplex; dado que es prácticamente imposible le transmisión y recepción simultanea en un ambiente WSN.

Existen diversos transceptores de bajo costo, los que incluyen todos los circuitos requeridos para transmisión-recepción a saber: modulación, demodulación, amplificador, filtros, mezcladores, etc. Para realizar una elección apropiada de transceptor debe entenderse apropiadamente sus principales tareas y características [128].

Este receptor debe ofrecer servicios a las capas superiores, fundamentalmente a la capa de Control de Acceso al Medio (MAC). Ciertas veces este es un servicio orientado a paquetes; y algunas veces el transceptor provee una interfaz de un byte (a veces solo un bit) al microcontrolador. La interpretación más simple de eficiencia energética del transceptor consiste en evaluar el consumo de energía para transmitir únicamente un bit.

La elección de la frecuencia de transmisión, es por supuesto, dependiente de los requerimientos y restricciones de los lugares de emplazamiento de las WSN. Un transceptor normalmente debe proveer varias frecuencias de transmisión (canales) de manera de poder resolver o aliviar problemas comunes de congestión en redes densas. Utilizando diferentes canales 
puede presentar escenarios de diferentes estados posibles de ahorro energético, en cada uno de los casos, el tiempo y la energía utilizada son los parámetros que deben evaluarse apropiadamente.

La frecuencia de la transmisión, el ancho de banda y la codificación utilizada determinan la tasa de transferencia bruta. Típicamente los transceptores soportan una o varias técnicas de modulación del tipo desplazamiento de amplitud ó frecuencia (ASK, FSK) ó similares. Algunos dispositivos proveen control directo de la potencia de transmisión, otro deben adicionar circuitos específicos con ese propósito. En estos dispositivos son deseables amplificadores con alta ganancia para así obtener buena eficiencia energética.

El alcance del equipo es considerado en ausencia de interferencia, dependiendo de la potencia máxima de transmisión, características de la antena, atenuación causada por el medio ambiente, que a su vez es bastante dependiente de la frecuencia de transmisión, del esquema de modulación/codificación utilizado, y de la tasa de error aceptable pretendida por el receptor.

Otras de las consideraciones a tener en cuenta en los transceptores de los nodos de WSN tienen que ver con el rendimiento de bloqueo, la emisión fuera de banda, la estabilidad de frecuencia y el rango de tensión admisible por el dispositivo.

En general los sistemas de radio pueden presentar en cuatro estados [104] de operación: transmisión, recepción, inactivo (idle) y zombi. En el estado de transmisión, la parte emisora del transceptor está activa y la antena irradia energía. En el estado recepción esta activa la parte recepción. En el estado inactivo, un transceptor se encuentra listo para recibir, pero no está recibiendo absolutamente nada en ese momento. En el estado inactivo, muchos de los elementos del circuito están activos, pero asimismo muchos otros están desconectados. En el estado zombi, 
casi todos los elementos del transceptor están desconectados, por cuestiones de ahorro energético. Lo negativo de esto es que se precisa mayor tiempo y energía para volver a un estado de los anteriores.

Normalmente la estructura de un transceptor consta del front-end de radiofrecuencia (RF) y la parte de banda base. El front-end de radio frecuencia realiza el procesamiento de la señal analógica en la banda de frecuencia correspondiente, mientras que el procesador de banda base procesa todas las señales en el dominio digital y se comunica con el procesador del nodo sensor, ó con otros circuitos digitales.

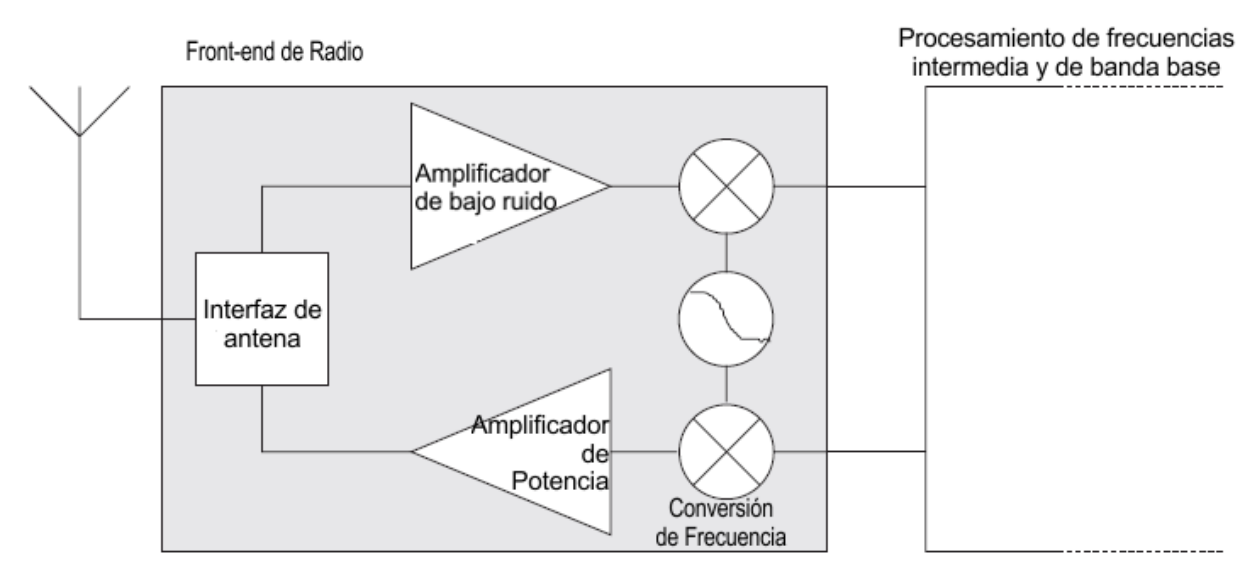

Ilustración 3.21. Esquema de front-end de radiofrecuencia

El límite entre los dominios analógicos y digitales lo conforman los convertidores Analógico/Digital (ADCs) y Digital/Analógico (DACs). El front-end de RF (ilustración 3.21) es la primera etapa de la interfaz comprendida entre las ondas electromagnéticas y el procesamiento digital de señales del transceptor [129] . 


\subsection{Resumen}

Los avances alcanzados en las tecnologías de hardware y de las redes de sensores inalámbricos han llevado a crear dispositivos diminutos de baja potencia, bajos costos y multifuncionales. Estos nodos colaboran entre sí para establecer una red de sensores, proveyendo acceso a la información en cualquier momento y en cualquier lugar; realizando las tareas de colección, procesamiento, análisis y diseminación de los datos. Con ello participan en la creación de ambientes inteligentes.

Se ha visto que los sensores pueden ofrecer servicios de vigilancia, pudiendo detectar y colectar datos referidos a indicios de fallas en maquinarias, terremotos, inundaciones y aún en casos de ataques terroristas.

Dado que las redes de sensores en general están formadas por un gran número de dispositivos, el costo de cada uno de ellos es importante al momento de determinar el costo total de la red a instalar.

En las redes de sensores, más que en las otras redes inalámbricas, la eficiencia del uso de la energía es crucial; en función del ámbito de instalación de estas redes y de las dificultades propias del recambio de baterías que se ha indicado en el capítulo.

Se han presentado a los equipos más representativos actualmente en el ámbito de las WSNs, considerando lo atinente a hardware ofrecido por las empresas, tanto en microprocesadores, memoria y radiofrecuencia. 
Capítulo 4 : Arquitectura de red en WSN 
a arquitectura de las WSN se basa en diferentes fuentes originarias. Existen diversos trabajos y publicaciones, que abarcan y profundizan lo referente a las redes ad hoc, móviles y auto organizadas [130], [131], [132], [133], [134], [135]. Si bien estas redes son utilizadas en distintos ambientes, las mismas comparten la necesidad de una organización descentralizada y distribuida. Desde una perspectiva diferente, las WSN son relacionadas al cómputo en tiempo real [70]; mientras en algunas publicaciones se relacionan a las WSN al cómputo peer-to-peer [136], a las redes activas, y a agentes de inteligencia móvil [137].

\subsection{Estructura de las redes de sensores}

Las redes de sensores pueden establecerse en diferentes estructuras, según se presentan en la ilustración 4.1

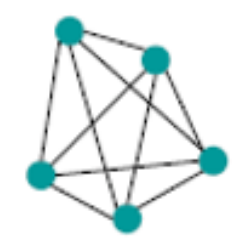

1 solo salto

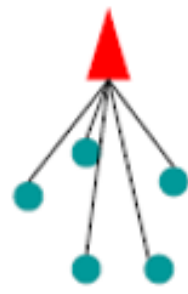

Estrella

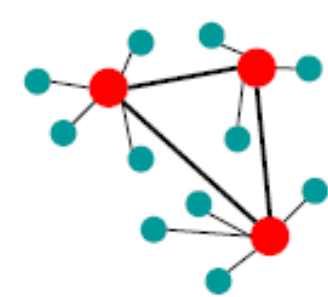

Cluster

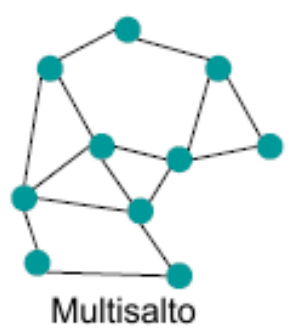

ad hoc

Ilustración 4.1 Estructuras de las WSN

Las redes de un salto (1-hop) se caracterizan por el hecho que todos los nodos pueden interactuar entre sí, siendo la limitante principal en estas redes en el alcance de transmisión del transceptor de los equipos. Cuando la estructura de la red es de tipo estrella (hub) todos los nodos se comunican con uno en particular; el que se conoce como nodo 
concentrador. Este es generalmente un nodo de mayor potencia de transmisión o cálculo comparándolo con los otros equipos de la red. Cuando en una red se selecciona y establece un segundo nivel de acuerdo al tipo de nodos, los que son seleccionados para ser utilizados en la red como nodos maestros por su potencia de cálculo, estamos en presencia de una estructura en cluster. Aquí los nodos sensores solo pueden comunicarse con los nodos maestros, mientras que los nodos maestros se comunican entre sí. La última estructura posible es la multisalto, donde los nodos se comunican con los nodos vecinos, y pueden comunicarse con nodos más lejanos utilizando a nodos intermediarios como routers para los paquetes de datos. Esta es la estructura de organización utilizada por todas las WSN, estableciendo la conectividad entre los nodos por medio de vínculos multisalto.

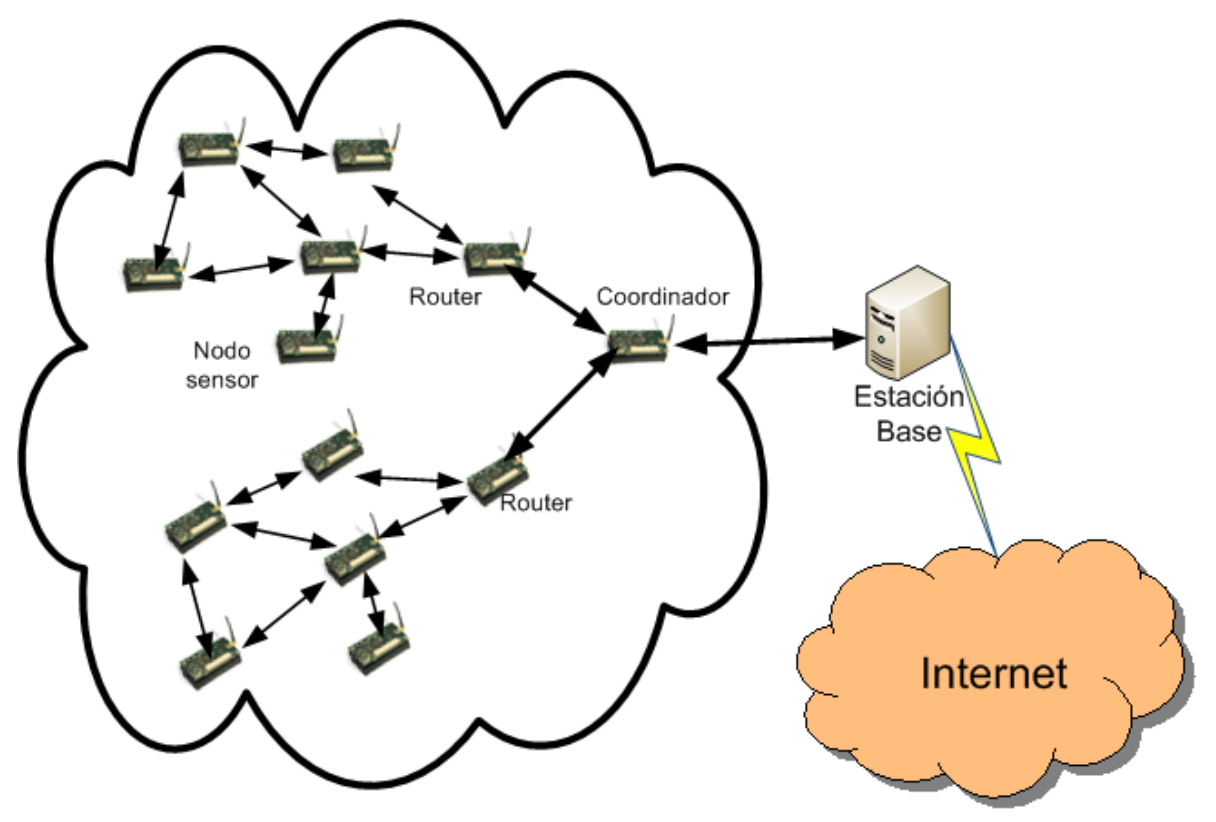

Ilustración 4.2 Arquitectura jerárquica en WSNs

Se considera que dos son las principales arquitecturas de las WSN; la arquitectura jerárquica y la distribuida. En la primera los nodos se 
clasifican según la tarea realizada en nodos comunes, routers y coordinadores; ver ilustración 4.2 .

En las arquitecturas distribuidas, no existe una infraestructura preestablecida, y la topología es desconocida, previa a la instalación de los dispositivos. La ilustración 4.3 muestra una arquitectura de este tipo.

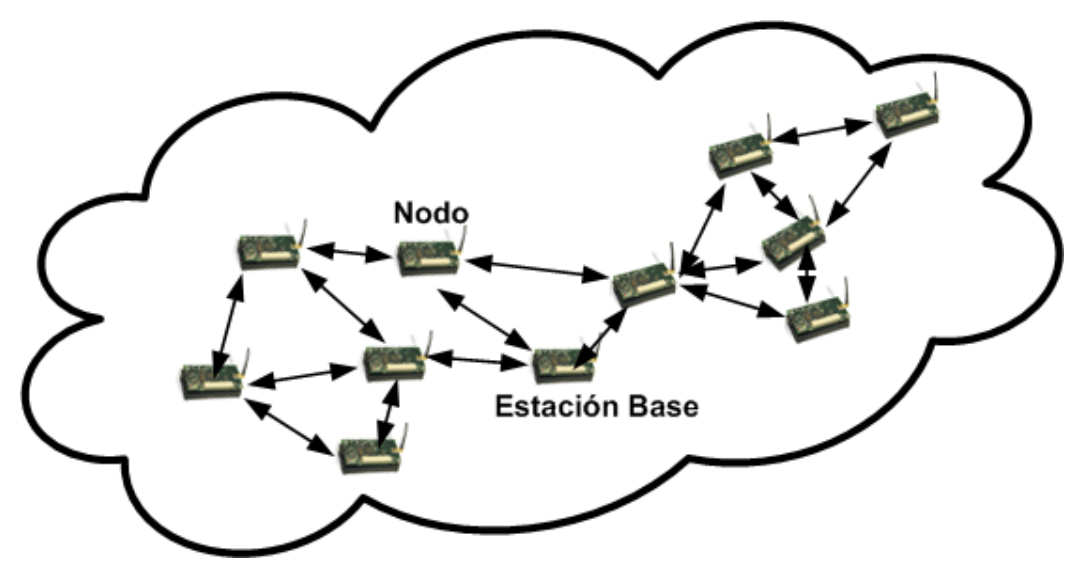

Ilustración 4.3 Arquitectura distribuida en WSN

\subsection{Roles de los nodos en las WSN}

Un nodo sensor de datos, es una entidad en la red cuya función principal es proveer información; ó también puede tratarse de un nodo actuador que provee retroalimentación sobre una operación realizada. Su actividad principal es realizar la medición y transferir la información.

Una estación base es la entidad en la red desde donde se solicita se obtenga la información sobre un evento determinado. Las estaciones base pueden pertenecer a la WSN (siendo uno más de los nodos sensores/actuadores), o puede tratarse de una entidad externa a la WSN. 
Una estación base normalmente está constituida por una PC ó una PDA, adaptada para interactuar con la WSN; pudiendo ser utilizada meramente como una puerta de enlace a una red mayor, tal como Internet. Por ello las solicitudes podrían realizarse no solamente de nodos locales de la red, sino desde lugares apartados. Los nodos colectores ${ }^{1}$ reciben la información desde distintos nodos, la almacenan, procesan y reenvían, conformando un paquete como una compilación de los datos de los sensores atendidos. Su función y objetivo en la red es la reducción del tamaño del paquete de datos, para así simplificar el transporte por los vínculos de la WSN. Esta tarea normalmente afecta favorablemente a parámetros de red como la eficiencia y la velocidad. Compárese el reenvío y la compilación de datos en el esquema presentado en la ilustración 4.4 .

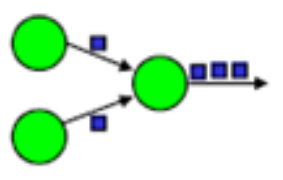

Reenvío
VS.

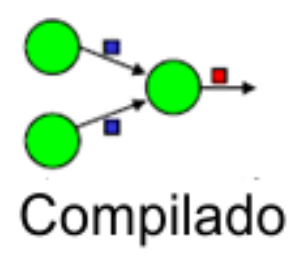

Ilustración 4.4 Diferencias operativas entre renvío y compilado de datos

Los nodos encaminadores, ó routers, reenvían los paquetes por rutas establecidas por algunas de las técnicas previamente seleccionadas para la red.

Finalmente encontramos a los sumideros ó sinks que son las pasarelas obligadas a redes de mayor tamaño o envergadura. Debido al tráfico de

\footnotetext{
${ }^{1}$ Aggregators
} 
información inherente en los sinks, es normal que en los vínculos que enlazan a estos equipos se presenten congestionamientos. En consecuencia, debido a los cuellos de botella, estos nodos son los que primeros salen de funcionamiento; afectando así al tiempo de vida útil de la red. Una de las soluciones de tipo práctica para evitar estos percances, es la utilización de múltiples sumideros, sumideros móviles ó agregación de datos. En la ilustración 4.2 se indica situaciones de congestión y paliativos a la misma.

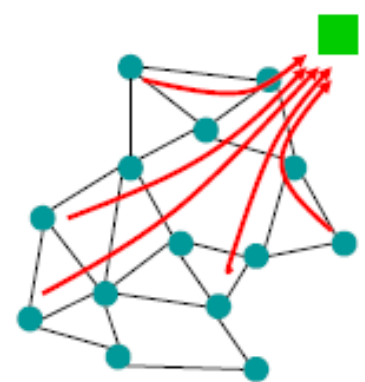

Congestión

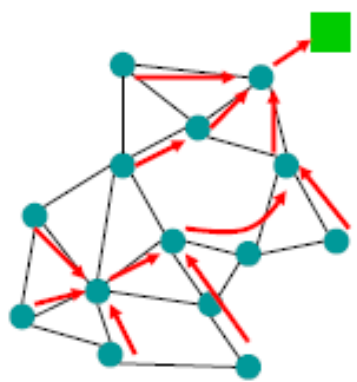

Agregación de datos

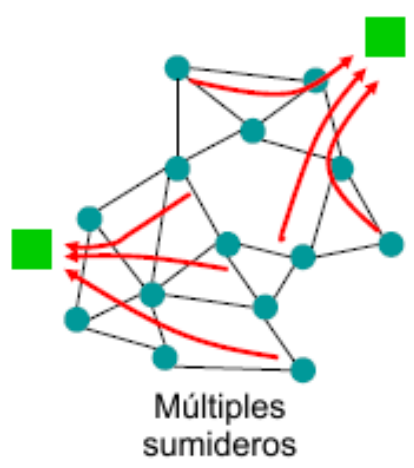

Múltiples
sumideros

\section{Ilustración 4.5. Escenario de congestión y paliativos}

Evaluar la arquitectura de la red es considerar, en cierto modo, los pro y contra de cada una de las técnicas de transmisión de la información: salto único o multisalto. La utilización salto simple o múltiple depende, como es de suponerse, del hardware, el escenario, la aplicación y el desempeño esperado de la WSN.

En ciertos trabajos se han establecido que un menor número de saltos más largos es más eficiente que un gran número de saltos de corta distancia [138]. En el estudio de comunicaciones radiales, se han realizado numerosos trabajos sobre la limitación inherente de la potencia de transmisión, limitando con esto la distancia posible entre emisor y receptor. Por este fenómeno la mayor parte de las veces no es posible 
interconectar al nodo origen con el sink; ya sea por las distancias entre ambas, por la superficie abarcada, ó por las dificultades geográficas del terreno donde se ha desplegado la instalación, con lo cual se produce la atenuación de las señales transmitidas.

Para evitar lo máximo posible esta situación las WSN implementan el sistema de reenvío de paquetes al sumidero por medio de nodos intermedios; utilizando la técnica multisalto [139], [140]. Esta técnica ha demostrado que mejora la eficiencia de la comunicación, dado que en todos los medios de transmisión, la atenuación de las señales de radio varía con el cuadrado de las distancias. Con ello, disminuyendo las distancias con multisalto se consume menos energía, que tratar de realizar el enlace por un vínculo de mayor distancia [141]. La energía irradiada para alcanzar una comunicación directa desde un nodo a una distancia $\mathbf{d}$ es $\mathbf{c} . \mathbf{d}^{\alpha}$, siendo c una constante y $\boldsymbol{\alpha} \geq 2$ es el coeficiente de pérdida en camino. Disminuyendo la distancia a la mitad se reduce esta

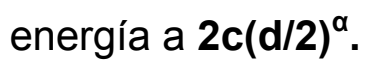

La técnica multisalto es de gran ayuda, cuando la instalación de la WSN se realiza en ambientes hostiles, o cuando las distancias entre los equipos es importante, necesitándose potencias de transmisión tales que trasgredan y no sean autorizadas por las regulaciones locales.

\subsection{Múltiples orígenes y sumideros}

La estructura establecida por los nodos de una WSN posee la particularidad de formar una vía de datos desde el fenómeno mismo hasta el observador. El objetivo es crear y mantener estas vías bajo condiciones dinámicas, manteniendo los requerimientos específicos: bajo consumo, baja latencia, alta precisión y ser tolerante a fallos. 
Como regla, los sensores no poseen un dirección universal que los identifique, sino que el interés es ubicar al set de sensores que están en condiciones de proveer lo datos requeridos por el observador. Asimismo a lo largo del camino del dato, cada nodo puede asumir un rol diferente en el procesamiento y diseminación del mismo.

\subsection{WSN estáticas y móviles}

Son varios los métodos conocidos Para construir y mantener el enlace entre el fenómeno y el observador se implementan varios métodos. Dependiendo de la dinámica propia de la WSN, éstas se clasifican en estáticas ó dinámicas.

La situación más simple es la presentada en las WSN estáticas, dado que no existe cambio de posición a largo del camino fenómeno-observador, como en el caso donde un set de sensores realiza la medición de la temperatura de una región. Los sensores realizan la comunicación local de los valores de la temperatura hasta un nodo seleccionado, el cual realiza la compilación de la información solicitada por el observador.

En WSN dinámicas puede ocurrir que el observador, los sensores y hasta el fenómeno mismo pueden ser móviles. Con esto, si un sensor que es parte del camino entre fenómeno-observador se mueve, puede producir un punto de falla en la WSN. Ante esta situación el observador, ó el sensor implicado en la caída del vínculo, deben intentar reconstruir la ruta de los datos.

\subsection{Movilidad}

El movilidad de los componentes de dentro de una WSN, es un factor importante en el fenómeno de comunicación, teniendo en cuenta que el tipo y calidad de la comunicación establecida en la WSN depende de la 
dinámica propia de la red. Cada uno de los escenarios planteados presenta particularidades diferentes en infraestructura, modelo de transporte de datos y de protocolos.

En las WSN se identifican tres tipos de movilidad [142], cada una de ellas involucrando a los participantes principales de estas redes: observador, sensores y fenómeno.

El primer caso, considérese al observador en un avión desde donde realiza las lecturas de los valores de cada uno de los nodos de la WSN. En esta situación el observador se está moviendo respecto a los sensores y al fenómeno que se está sensando a nivel del suelo. Cuando un nodo se mueve respecto al observador y cada uno de los restantes nodos, estamos en presencia de movilidad de equipos. Como ejemplo tómese una WSN en una flota de taxis, donde a medida que los taxis se mueven van sensando y comunicando la información referente a las condiciones de tráfico a los otros taxis en el rango de alcance.

La tarea insignia de las WSN desde sus orígenes ha sido el monitoreo y detección de animales (fenómenos móviles). En este ambiente, dependiendo de la densidad de sensores; la medición del fenómeno puede ser inadecuada si se detecta en diversos los sensores al mismo tiempo. Los sensores que detecten la actividad debe ser aquellos vecinos al evento, el despierta con su movilidad una serie de dispositivos a los largo de su ruta presentando un fenómeno conocido como "wakeup wavefront". Por ello la infraestructura de la red de la WSN debe ser orientada a eventos.

\subsection{Objetivos de optimización}

Las WSN normalmente son utilizadas en zonas donde las tareas realizadas se consideran peligrosas para la salud, o pueden estar 
desplegadas en zonas inaccesibles donde existen riesgos de perder la vida. Dado que los nodos son alimentados por baterías implica considerar el menor consumo energético al desarrollar sus actividades; para así prolongar al máximo su vida útil. Además debe considerarse un prerrequisito mantener la confiabilidad del dato transmitido por la red. Ambos puntos, el consumo energético y a la confiabilidad, se han destacado en los estudios e investigaciones como los temas más importantes en la optimización de modernos sistemas de comunicación basados en WSN.

Frente a estos escenarios y aplicaciones, siempre es posible encontrar diferentes tipos de redes capaces de satisfacer las necesidades de un proyecto. El primer interrogante que se debe atender tiene que ver con la identificación de los nodos, ó en su defecto de las redes. Esto es necesario para poder lograr la integración efectiva a diferentes redes de mayor categoría, tal como Internet. La pregunta angular en definitiva es: ¿Se debe asignar a cada nodo una dirección IP; ó a se debe identificar solamente a cada red de sensores?

La otra cuestión tiene que ver con la integración de la red de sensores a la web, definiendo si una red de sensores inalámbricos es, o no; un servicio web. En el caso que así lo consideremos, es necesario definir cuál de las WSN cumple con los requisitos necesarios; y sobre todo, como avanzar con la heterogeneidad de los datos.

En todos estos planteos debe considerarse como optimizar la red, como comparar estos resultados, decidir cuál es el mejor modelo que soporta una aplicación dada, y en definitiva como convertir los objetivos difusos de los objetivos de optimización en aspectos prácticos y valores mensurables de comparación. Es imposible considerar todos y cada uno de los aspectos, aunque alguno o pocos de ellos son más que evidentes. 


\subsubsection{Calidad de servicio}

Las WSN son diferentes a las otras redes convencionales de comunicación, principalmente por el tipo de servicio que ofrecen. Las WSN realizan casi siempre la misma y única tarea: mover bits de un lugar geográfico a otro.

La calidad de servicio en estos casos se considera un atributo de bajo nivel, donde debemos considerar ancho de banda, retardo, jitter, tasa de pérdida de paquetes. Como un atributo subjetivo o de alto nivel, podemos referirnos a la calidad percibida por el usuario en una comunicación de voz o una transmisión de vídeo. Mientras el primer tipo de atributos es aplicable a un cierto tipo de redes inalámbricas de sensores; el segundo claramente no lo es, pero es la que debe ser principalmente considerada.

Posiblemente, los servicios de una WSN sobre los que se debería ofrecer calidad de servicio (QoS), son las desarrolladas en el contexto de las aplicaciones multimedia. Una red de sensores inalámbricos puede convertirse rápidamente en una red multimedia de sensores inalámbricos, con aplicaciones capaces de realizar seguimiento, domótica y monitoreo ambiental [143].

Las WSN se componen de un gran número de unidades sensores distribuidas en el terreno de acuerdo al interés despertado por el evento a ser sensado. Existen innumerables aplicaciones de las WSN, por lo que es imposible considerar todos y cada uno de los requerimientos de QoS de las mismas.

En la ilustración 4.6 se observa un enfoque holístico de QoS en WSN, apuntando al concepto de invisibilidad que promoviera Mark Weiser. 


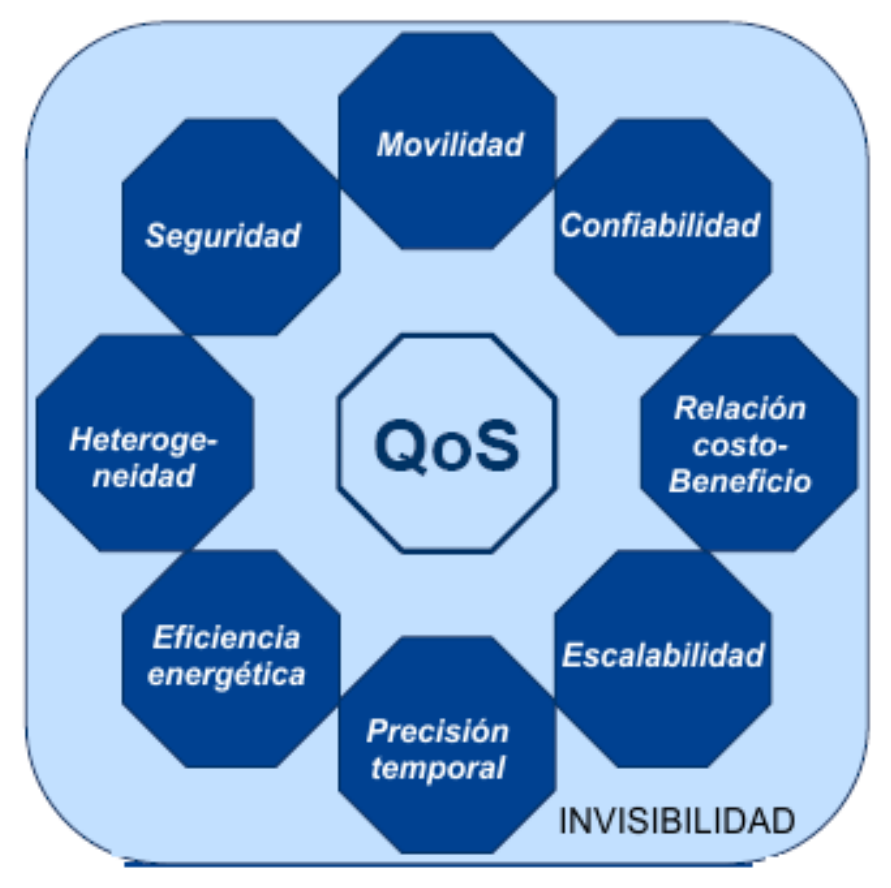

Ilustración 4.6 Enfoque holístico de la calidad de servicio

Los requerimientos de QoS en una WSN pueden llegar a ser totalmente diferentes a los parámetros tradicionales considerados en redes end-toend, los que no podrían adoptarse directamente. Por ello es necesario establecer nuevos parámetros de QoS, los que tendrán razón de ser en el ámbito de las WSN.

El propósito de los parámetros considerados, apuntan a como la red subyacente puede proveer calidad de servicio a las aplicaciones WSN, por medio de parámetros que pueden especificar las necesidades de la infraestructura de red y poder mensurar la calidad de servicio apropiadamente. Para ello se describen dos perspectivas de QoS en WSN [144]. En primer lugar el QoS especifico para WSN, considerando parámetros como cobertura, exposición, errores de medición y número óptimo de sensores activos. Estos parámetros influyen indiscutiblemente 
en la calidad de las aplicaciones. Otra consideración se realiza al QoS de red, que considera como la red de comunicaciones es capaz de distribuir los datos obtenidos por los sensores en una red bajo parámetros de QoS, cuando los recursos propios de la red no abundan. Si bien no se pueden analizar cada una de las situaciones posibles, es dable de realizar una clasificación clasificando a las aplicaciones que realizan requerimientos comunes a la red.

Los modelos de despacho son orientados a eventos, orientados a consultas y de despacho continuo.

La mayoría de las aplicaciones orientadas a eventos son interactivas, en tiempo real, es una misión crítica y no end-to-end. Los datos que fluyen desde los sensores son altamente correlacionados y redundantes, donde el tráfico generado por un sensor común puede ser una acción de baja intensidad de respuesta comparada con las generadas por los sumideros o actuadores, la que debe ser distribuida rápida y confiablemente.

Cuando las aplicaciones de la WSN son orientadas a consultas, son tolerantes a retardos ante requerimientos específicos, son misiones críticas y no end-to-end, los datos son provistos por los sinks, y una consulta puede utilizarse para reconfigurar nodos. Los comandos emitidos desde un sumidero constituyen tráfico unidireccional y requiere alta confiabilidad.

En el modelo de despacho contínuo, los nodos envían continuamente la información obtenida al sink a una velocidad prefijada. Cuando se trata de imágenes, audio o video en tiempo real, es considerado como restringido en retardo, con ciertos requerimientos de ancho de banda. Es capaz de soportar perdida de paquetes hasta un determinado punto, y las aplicaciones no son end-to-end. 
Dado entonces que la calidad de servicio en WSN se establece sobre parámetros colectivos, no end-to-end; lo que deben considerarse es la importancia de la latencia colectiva, pérdida de paquetes colectivos, ancho de banda colectivo y capacidad total de transferencia de la red.

Al igual que en las redes tradicionales, los atributos de alto nivel de QoS, dependen de la aplicación. Alguna de las posibilidades generales de parámetros de QoS son la probabilidad de detección y reporte de un evento, error en la clasificación del evento, retraso en la detección del evento, reportes extraviados, precisión en aproximación y precisión en rastreo de individuos.

\subsubsection{Heterogeneidad}

La heterogeneidad es importante dada la existencia de diferencias en: software y hardware de red, protocolos de comunicaciones entre sensores y actuadores, protocolos de comunicación de alto nivel, herramientas de planificación de red, calibración y diversidad de diseño de los nodos, sistemas operativos, lenguajes de programación y en middleware.

\subsubsection{Eficiencia energética}

La energía es la piedra angular en la investigación y desarrollo de las WSN. Está claro que si dispusiéramos energía en cantidades arbitrarias, todos los fundamentos y límites de QoS definidos anteriormente podrían alcanzarse sin inconveniente. Eficiencia energética es un término utilizado para instalar bajo un paraguas una cierta cantidad de parámetros que deben ser considerados cuidadosamente, algunos de ellos son: energía por bit recibido correctamente, energía por evento reportado, relación retardo/energía, vida útil de la red. 


\subsubsection{Precisión temporal}

Este concepto tiene que ver con comportamiento respecto al tiempo del sistema en su conjunto, reflejado en propiedades como capacidad de transporte, tasa efectiva de transmisión bits, retardo de mensajes, diferenciación de tráficos, clases y prioridades de tráfico, etc. Estos parámetros pueden ser balanceados con otros parámetros de QoS. El aumento de la capacidad final de transporte de datos de la red (throughput) debería considerar la actualización de equipamiento para permitir el incremento de la tasa de transporte de bits; ó aumentar los ciclos de operación, conllevando a un consumo mayor de energía.

En un entorno en el cual muchas de las actividades implican la utilización en forma cotidiana de aplicaciones en tiempo real, los parámetros de la precisión temporal asumen importancia relevante. Estas aplicaciones, por supuesto, precisan de sistemas operativos, lenguajes de programación y protocolos de comunicación adaptados a las necesidades del tiempo real.

\subsubsection{Escalabilidad}

La escalabilidad es importante al momento del diseño de protocolos de comunicación eficientes para WSNs. Un protocolo debe adaptarse y escalar a los cambios en la topología de red, adaptando su comportamiento a medida que la red crezca en cantidad e nodos, ó el tráfico a través de los vínculos se incremente. Esto se logra con la utilización de algoritmos distribuidos y localizados, en los cuales los nodos se comunican solamente con equipos vecinos similares. Los sistemas centralizados son descartados, descartando con ello el punto único de falla.

La escalabilidad se entiende como la capacidad de un sistema de adaptarse fácil y rápidamente a variaciones en: el número de nodos, 
densidad espacial de equipos y rango de cobertura. En redes donde el número de nodos es de varios cientos de nodos, la capacidad de medida aumenta linealmente con el número de equipos en la misma, no así la capacidad de comunicación; considere que mil nodos propagando un mensaje de un milisegundo, se propagará por la red en aproximadamente veinte minutos. Por ésta razón la utilización de simuladores de WSN consisten en una herramienta fundamental para el estudio de la escalabilidad [145].

\subsubsection{Relación costo-beneficio}

El costo de un sistema debe incluir los siguientes puntos: diseño y desarrollo, hardware, instalación y distribución en campo, revisión y mantenimiento. Los desafíos para el futuro consisten en obtener nodos de costo menor a un dólar por unidad, cuando en la actualidad los mejores precios encuentran entre 10 y 50 dólares americanos. Se estima que la baja de los costos de los nodos será por efecto de la producción masiva de equipos, y a la adopción de procesos más económicos de diseño y producción; como en los avances de la miniaturización de componentes como MEMS.

\subsubsection{Confiabilidad}

En los distintos ambientes de aplicación de las WSN pueden encontrarse condiciones desfavorables de ambiente, como condiciones indeseadas de vibraciones, golpes, temperaturas y presiones extremas, humedad, agua, polvo; y otras fuentes de interferencia electromagnética.

La transmisión de datos en una WSN, en grado mucho mayor que en redes cableadas, es propensa fallas e impredecible. La razón de ellos es que los equipos son construidos con elementos frágiles y de capacidades limitadas. En las redes multisalto, típicas en las WSN, se observan 
frecuentemente errores en los vínculos por interferencia, obstáculos, ambiente o movilidad. También se producen congestionamientos debido al tráfico por ráfagas, y con ello la pérdida de paquetes de información. Con el fin de evitar estos inconvenientes los equipos deben ser los más robustos y confiables que sea posible, para poder operar en las condiciones adversas mencionadas, reduciendo al máximo el mantenimiento y mantener la operatividad de la red el mayor tiempo posible.

\subsubsection{Robustez}

En relación a QoS y los requisitos de escalabilidad, las WSN deben demostrar buena robustez. La red no debe salir de operación por más que varios nodos agoten su batería ó pierdan conectividad por interferencias electromagnéticas, o por agresiones propias del ambiente. Estas fallas deben ser compensadas en la WSN buscando rutas más convenientes. La tarea de ajuste de robustez en WSNs no es una tarea simple, ya que depende de modelos de falla tanto en nodos, como en enlaces.

\subsubsection{Movilidad}

En las WSN son típicos los fenómenos de movilidad de los diferentes actores, los que pueden ser vehículos, equipos, animales, personas, fluidos, etc. La WSN debe ser capaz de considerar a la movilidad de manera de considerar el mantenimiento y restablecimiento de la conectividad de la red, mejorar la cobertura, balancear el consumo energético, adaptarse a cambios dinámicos, extender su vida útil, incrementar el QoS en regiones críticas, mejorar y extender los límites de la WSN, y por supuesto; incrementar la satisfacción del usuario. 


\subsubsection{Seguridad}

La seguridad en WSN es una tarea mucho más compleja que las soluciones actuales para sistemas de escritorio y empresariales. Las WSN son usualmente sistemas a gran escala, inalámbricas, modulares, heterogéneas, desatendidos, en ambientes hostiles y de naturaleza dinámica. Estos pueden alcanzar límites mas allá de los ambientes controlados, donde no existen controles centralizados ni autoridad confiable [101].

Por lo anterior las WSN precisan de consideraciones particulares de seguridad, teniendo en cuenta el tipo de nodo y los mecanismos de adaptación, lo que se conoce como reconfigurabilidad.

\subsection{Principios de diseño de WSNs}

Todos los elementos que hemos tratado hasta ahora no son lo suficientemente explícitos como para indicar el "como" diseñar una red de sensores, si bien existen algunos trabajos sobre el tema [146], [147]; ninguno de ellos se explaya suficientemente.

En el diseño de una WSN, y para cumplir con los requerimientos de QoS, Robustez y escalabilidad, indefectiblemente tenemos que pensar en una organización distribuida. Las desventajas del sistema distribuido se presentan siempre como puntos de falla, siendo realmente difícil de estas fallas en redes basadas en radio, ya que cada uno de los equipos que forma parte de la WSN posee una capacidad de comunicación limitada. Los nodos entonces utilizan protocolos y algoritmos distribuidos de manera de poder organizarse.

Una de las estrategias, es utilizar la organización centralizada en ciertos sectores de la red de acuerdo a las necesidades, considerando que esta forma de organización ciertas veces produce un mejor aprovechamiento 
de los recursos de la red. Ejemplo de ello es la organización del acceso al medio, resultando en definitiva una jerarquía.

En una red organizada de la forma distribuida, los nodos no solamente se dedican a pasar paquetes hacia sus vecinos, sino deben tomar decisiones que hacen al rendimiento, debiendo realizar un procesamiento de la información "red adentro". Esta técnica es abierta, sin límites o concepciones; y utiliza cualquier técnica que mejore la aplicación del sistema.

Probablemente la mejor técnica de procesamiento "red adentro" es la técnica de agregación. Se denomina así porque en los nodos intermedios, entre el origen y el sink, la información se va agregando y procesando resguardándola en forma condensada. Obviamente la agregación de datos sigue características determinadas, la más importante de ellas es el poder descifrar la composición de la información.

Las funciones que pueden ser aprovechadas y maximizadas con la agregación de datos son el promedio, conteo y mínimo [148]; mientras que no resulta el agregar el valor medio. En la ilustración 4.7 se muestra un esquema y el resultado de la agregación.

En la semifigura izquierda de la ilustración 4.7 se ilustra a varios sensores transmitiendo sus lecturas por medio de comunicación multisalto. En total son necesarios 13 mensajes. En la figura se indica el número de mensajes transportados por cada enlace. A la derecha del mismo grafico, se muestran resaltados los nodos donde se realizan agregación, observándose que solo son necesarios 6 mensajes luego de la agregación de datos. 

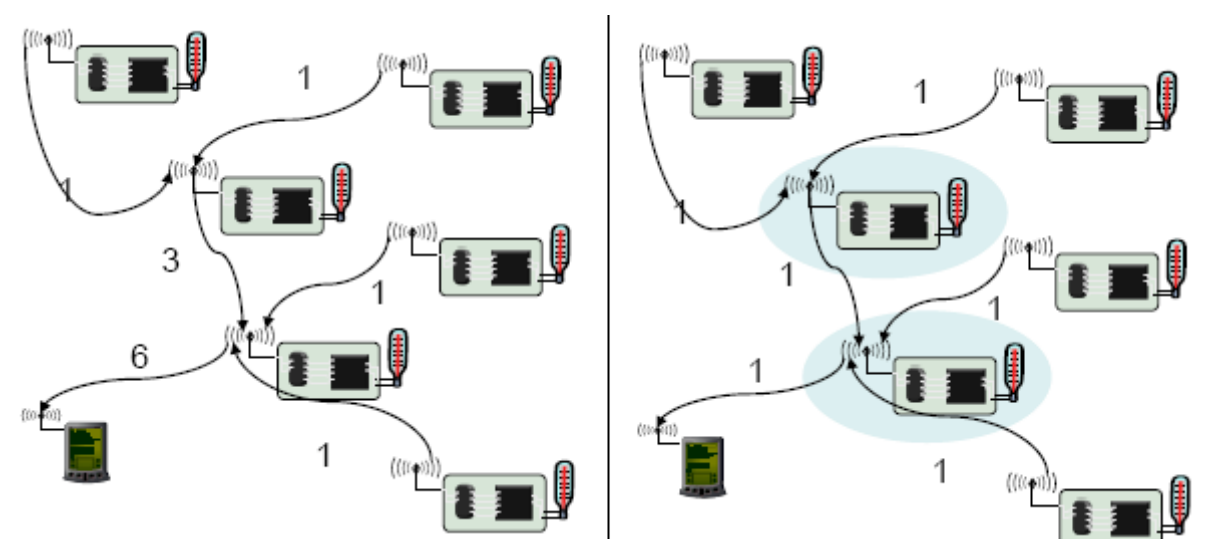

Ilustración 4.7. Ejemplo de agregación adaptado de Holger and Willig [128]

Entre los principios de diseño deben considerarse el codificado y la compresión distribuida, dado que según el teorema de Slepian y Wolf [149], las lecturas efectuadas por sensores adyacentes deben ser necesariamente correlacionadas respecto a los valores reportados. Estos hechos son utilizados por las WSN para realizar correlaciones espacial y temporal. Pueden considerarse también la implementación de otros paradigmas ó modelos computacionales, tales como código móvil ó redes basadas en agentes.

Si bien la idea es lograr una representación compacta del programa ejecutable que sea lo suficientemente pequeño para ser enviado de nodo en nodo, en una determinada secuencia [150]. En los últimos años se ha considerado a estos sistemas de forma similar a sistemas biológicos conocidos como "inteligencia de enjambre" [151], [152], [153]; dado que son un grupo de entidades simples que trabajan en conjunto para lograr un bien común. 


\subsection{Centralidad de datos}

En este nuevo siglo, la computación y las comunicaciones tienden a dejar de lado los dispositivos de propósito general, focalizándose en la necesidad de los usuarios de equipos específicos, fáciles de utilizar, libres de mantenimiento, portables, ubicuos y muy confiables. Estos dispositivos, si bien no son de recursos escasos como en el caso de los nodos de las WSN, encuentran otras limitaciones como lo son el tamaño, factor de forma y consumo energético.

Lo anterior no es un problema privativo de las redes ad hoc, pero infiere que se debe redefinir todo lo referente a transacciones en la red, convirtiéndose en un desafío el desarrollo de servicios distribuidos. La tendencia es que la transferencia de datos desde las interfaces de usuario a los servicios, y viceversa, sean totalmente transparentes, guiados por las tareas más que por comandos explícitos. El modelo puede ser utilizado en ciertas ocasiones, por ejemplo cuando un nodo pretende comunicarse con otro con el fin de enviarle un mensaje breve específico. La gran mayoría de las comunicaciones en la Internet de hoy redes no encaja en este comportamiento. Lo que pretende cada nodo casi todo el tiempo es descargar, publicar, ó difundir un determinado dato.

Esta situación se presenta en WSNs densamente desplegadas, de tal manera que un determinado evento puede ser reportado por diferentes nodos simultáneamente, no importando que nodo específicamente esté reportándolo. Cuando la identidad de los nodos no es cosa importante, y los datos devienen centro de atención, se denominan redes data-céntricas

En redes tradicionales, donde lo importante son los nodos emisores y receptores, dado que las transacciones de red se direccionan a nodos específicos, se denominan redes nodo-céntricas. 
Las WSNs son data-céntricas, ya que la importancia esta focalizada en los reportes producidos por esta (datos) y no en los nodos emisores o receptores.

A esta altura es menester preguntarse cuáles son las principales cuestiones de diseño en redes data-céntricas. Debemos considerar tres puntos fundamentales:

- Superposición de redes y tablas de hash distribuidas. En redes peer-to-peer como las WSN, la solución es la superposición de redes manteniendo una tabla de hash distribuida [154], [155], [156]. A pesar de estas similitudes, existen diferencias sustanciales. El comportamiento agnóstico de las redes IP respecto a no considerar los múltiples saltos como factor de diseño no es aplicable en WSN [128]; dado que cada salto durante la transferencia de información significa un overhead considerable. En otros trabajos se tienen en cuenta la topología de las redes establecidas [157], [158]; y la posición de los nodos [159], [160] al superponer las redes.

- Publicar/Subscribir: Determinar la ubicación temporal, como la identidad del sumidero (sink) que solicita algún tipo de información no es una tarea fácil en una WSN bajo régimen de protocolo petición/respuesta. En WSN se implementa el método publicar/ subscribir [161], [162]. Cada nodo interesado en algún tipo de dato, debe subscribirse al mismo. Cada nodo puede publicar el dato que puede ser accedido. Una vez que ciertos tipos de datos son publicados, la información es remitida todos los subscriptores. Considérese que la subscripción y la publicación pueden estar desfasadas en el tiempo, y los equipos intervinientes pueden ser desconocidos entre sí.

- Bases de datos: Algunos autores consideran a las WSN como bases de datos dinámicas [163]. Esta visión encaja perfectamente 
con la idea de utilización de redes organizadas en forma datacéntricas [164]. En la ilustración 4.8 se muestra este esquema de trabajo, donde se puede observar que consiste de sistema más complejo que el sistema centralizado de DB, posee costos más bajos de comunicación y se comporta en forma data-céntrica, es decir peer-to-peer

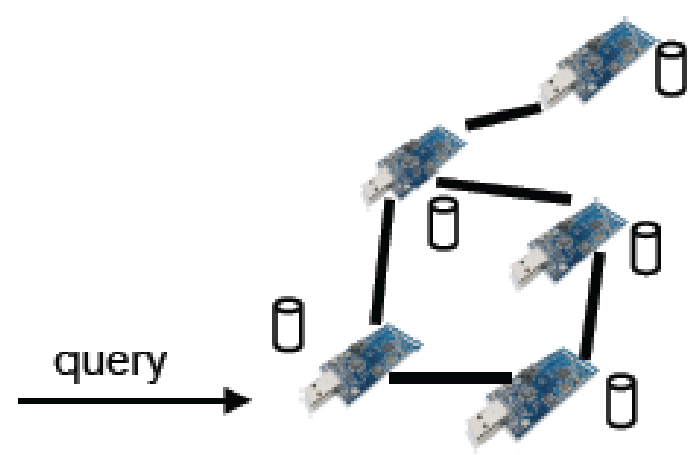

Ilustración 4.8 WSN como base de datos

\subsection{Otros fundamentos de diseño de WSN}

Lo referente a la ubicación es un dato normalmente requerido en muchas aplicaciones, para así determinar la ubicación de los nodos sensores geográficamente. Con la utilización de la información de localización se mejora considerablemente la performance de los protocolos de comunicación, logrando a una mejora en el rendimiento energético total.

Los patrones de actividad de las redes de sensores inalámbricos son totalmente diferentes de los otros tipos de redes. Cuando se detecta un evento, el mismo puede ser captado por numerosos sensores de la cercanía. Por ello los protocolos deben estar diseñados de tal manera de poder manejar e identificar estas ráfagas de datos producidos 
puntualmente, siendo capaz de conmutar desde un estado de reposo absoluto al de máxima actividad.

Debe considerarse que los sensores pueden ser heterogéneos por construcción ó por evolución. En uno u otro caso la heterogeneidad es tanto una desventaja como una oportunidad para la WSN.

\subsection{Optimización de la pila de protocolos}

La pila de protocolos es una implementación particular de software de red de computadoras, a la que normalmente la denominamos suite de protocolos. La suite es la definición de los protocolos, y la pila es software de implementación de los mismos.

Los protocolos individuales en una suite son generalmente desarrollados con un propósito particular. Dado que cada modulo del protocolo se comunica con otros dos, se imaginan a los mismos como capas en una pila. Las capas más bajas interactúan con el bajo nivel, es decir con el hardware. Cada capa superior, añade características a su comportamiento. Las aplicaciones de interfaz con el usuario tiene lugar en las capas más altas de la pila, normalmente dividida en tres secciones: medios, transporte y aplicaciones. Una plataforma ó sistema operativo debe poseer dos interfaces bien definidas; una entre el medio y la capa de transporte, y otra entre la capa de transporte y la aplicación. El transceptor de un nodo en una WSN no tiene idea de la red, siquiera del concepto de comunicación con otros nodos. Esas tareas son realizadas por la pila de protocolos de comunicación.

La pila de protocolos ha sido utilizada por décadas proveyendo funcionalidad y estructura formal a los subsistemas de comunicación por medio de la utilización de las capas múltiples. El numero, contenido, y función de las capas difiere entre los diferentes protocolos [165]. Cada 
capa de la pila de protocolos implementa una serie de tareas en la red, ofreciendo servicio a las capas inmediatamente superiores. Utilizando la pila de protocolos, pueden implementarse diferentes aplicaciones en la misma capa, con un mínimo impacto en la demás capas.

La mayoría de los protocolos de comunicación modernos han modificado el modelo básico de referencia de Interconexión de Sistemas Abiertos (OSI), para así adaptarlo a sus necesidades, como ZigBee resumió a las capas superiores en su capa de aplicación.

\begin{tabular}{|c|}
\hline Application \\
\hline Presentation \\
\hline Session \\
\hline Transport \\
\hline Network \\
\hline Data Link \\
\hline Physical \\
\hline
\end{tabular}

OSI Basic

Reference Model

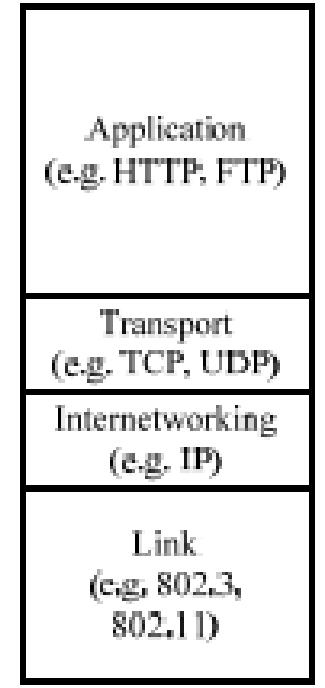

Internet Reference Model

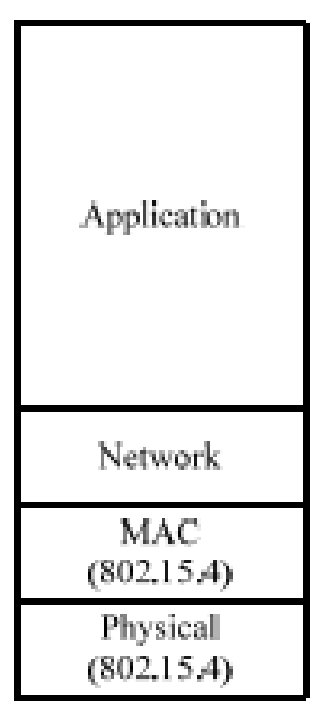

ZigBee

Ilustración 4.9 Pila de protocolos de Modelo Básico de Referencia OSI, TCP/IP y ZigBee

Son diversos los enfoques en el estudio de las pilas de protocolos (ilustración 4.10), algunos las consideran como un árbol de protocolos [166], según el cual para que una WSN funcione eficientemente, los protocolos utilizados por las diferentes capas deben tratar de alcanzar el 
mismo objetivo; algo totalmente diferente a lo propuesto por la pila de capas por localización [167] ya que realiza abstracciones apropiadas, utiliza terminología común y provee interfaz de programación de aplicaciones bien definidas [67].

Los protocolos realizan las tareas normales y comunes en todas las redes, el permitir la comunicación entre las diferentes capas de la pila.

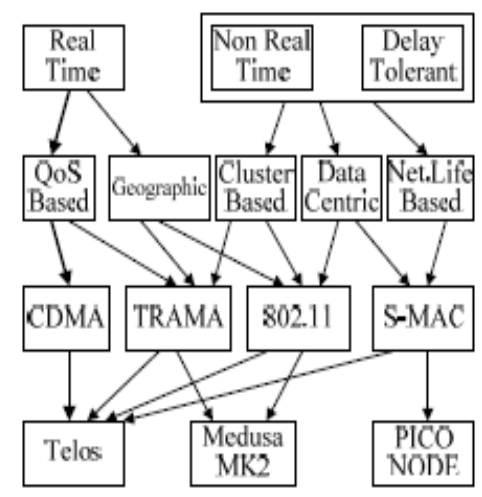

(a)

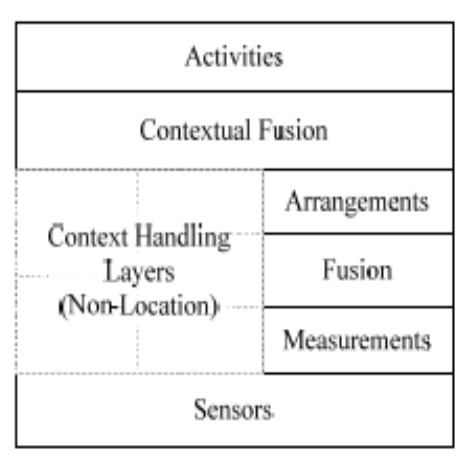

(b)

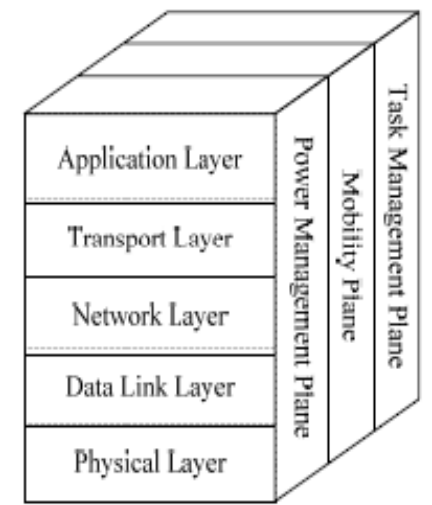

(c)

Ilustración 4.10 Pilas de arquitecturas existentes para WSN a) arbol de protocolos b) localización c) tridimensional

\subsection{Interfaces de servicio en WSN}

En redes tradicionales el programador de la aplicación puede acceder a los servicios de la red a través de una interfaz comúnmente aceptada, los sockets [168]. Esta interfaz contiene disposiciones claras sobre cómo manejar las conexiones, cómo enviar y recibir paquetes, y la forma de preguntar sobre la información del estado de la red.

En las WSN se produce otra forma de interacción, que es la de cruce de capas, indicada en la ilustración 4.11. Esto contradice la modularidad de las capas de acuerdo a la teoría de redes convencional, pero se 
corresponde con una promesa de mejora considerable de la performance de las redes.

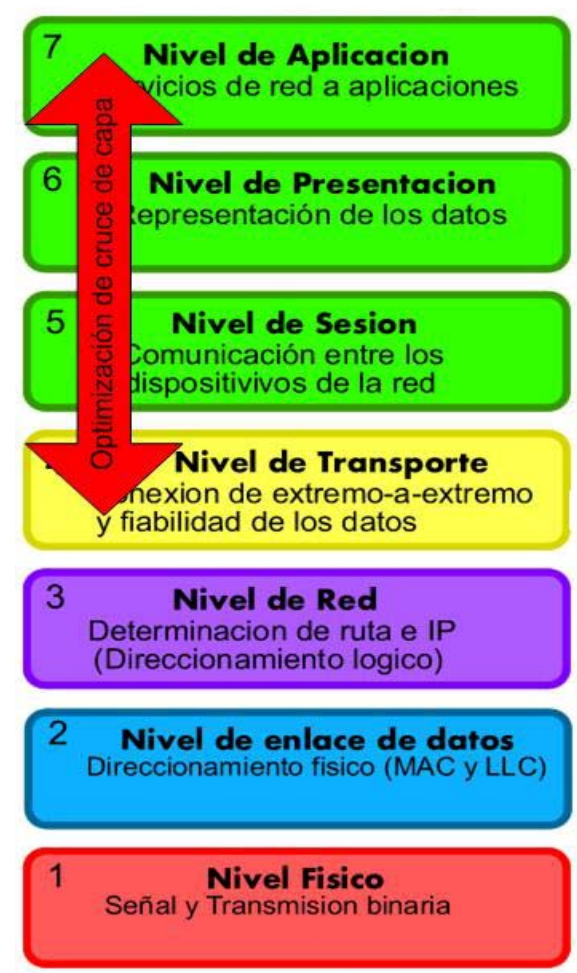

llustración 4.11 Protocolos de cruce de capas para WSN

Sin embargo, existe la opción de diseño entre el tratamiento de la aplicación como cualquier otro componente y el diseño de una interfaz de servicio que hace que todos los componentes en su totalidad, sean accedidos de forma normalizada. Estas dos opciones se describen en la Ilustración 4.12. En la segunda forma, la interfaz de servicio propuesta puede ocultar considerablemente la complejidad de la red, y convertirse así en un "middleware" del sistema. 


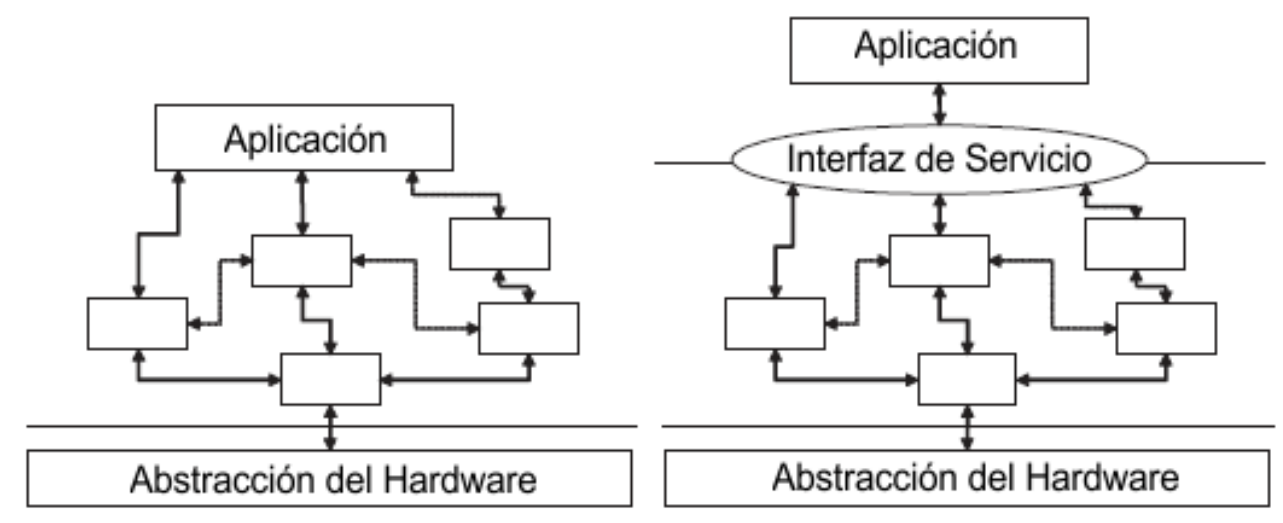

llustración 4.12 Dos formas de interfaz entre una aplicación y la pila de protocolos

\subsection{Resumen}

Tanenbaum [169] ha expresado que "cada vez que aparece un tipo nuevo de computadora, la misma pasa por todas las etapas del desarrollo de sus antepasadas, empezando a ser programadas en Assembler, hasta llegar a los lenguajes de alto nivel". Este patrón es también observado en el desarrollo de las WSN.

En este capítulo se mencionan las características de las aplicaciones y middleware para WSN, como también los retos a los que se enfrenta la tecnología. El integrar procesamiento simple, almacenamiento, medición, capacidad de comunicación en dispositivos tan pequeños, abre la puerta a un mundo de nuevas aplicaciones. Lo concerniente a la arquitectura de red en WSN es una lucha para encontrar un modelo de negocio que pueden convertir las visiones brillantes, en algo próspero, real y útil. Esta lucha se puede ganar mediante la aplicación de ideas creativas de la tecnología subyacente, en el supuesto de que esta tecnología y sus habilidades, así como las deficiencias y limitaciones son bien entendidas. 
Se ha tenido en cuenta en el desarrollo del capítulo, la estructura de las redes y los roles de los nodos que la integran, representando las diferentes topologías posibles de encontrar en la práctica. Se intenta fundamentar la importancia de las WSN cuando se consideran escenarios con movilidad, de nodos o de fenómenos; indicando asimismo los objetivos de la optimización y los principios de diseño de las WSN.

El comprender este nuevo tipo de redes es un reto formidable. Una característica clave es la necesidad de entender los problemas de muchas áreas diversas, que van desde aspectos de bajo nivel de comunicación de hardware y de radio a los conceptos de alto nivel, como bases de datos o middleware, ó las aplicaciones en sí mismas.

Al final del capítulo se considera una posibilidad en el entorno de las WSN, a contracorriente de lo estudiado en otros tipos de redes, donde los servicios prestados por las diferentes interfaces se encuentran en capas no adyacentes, por medio de un proceso de cruce de capas. 


\section{Capítulo 5 : Las simulaciones}


a simulación por computadora ha permitido a los científicos e ingenieros experimentar fácilmente con ambientes virtuales, alcanzando un nuevo nivel de detalle el análisis de las aplicaciones naturales y artificiales; que fuera desconocido en las primeras etapas del desarrollo científico. Además proporciona una gran ayuda en el diseño y análisis de aplicaciones complejas.

Es conocida la complejidad de modelar analíticamente a las WSN, dado que se tiende a realizar análisis simplificados. Toda simulación requiere de un modelo apropiado basado en fundamentos teóricos y sobre todo, de fácil implementación práctica [170], dado que los resultados de la simulación se extrapolan del escenario particular de análisis, con determinadas presunciones, que ciertas veces no encierran al comportamiento real de las WSN. Con ello se compromete seriamente la credibilidad de las simulaciones.

Donde realmente las simulaciones demuestran su utilidad es en el análisis de escalabilidad y en cuestiones de performance, parámetros difíciles de medir en campo debido al gran número de nodos que normalmente conforman la WSN. Todo estudio o investigación requiere normalmente un alto nivel de experimentación como forma de validación de las simulaciones.

La programación de los nodos de una red de sensores inalámbricos normalmente requiere del conocimiento de sistemas embebidos, y del conocimiento del lenguaje específico basado en texto, que fuera seleccionado por el proveedor. El software desarrollado, o adaptado para las WSN; debe ser probado a fondo antes de realizar implementaciones en el mundo real, fundamentalmente debido a que los nodos sensores no ofrecen interfaces amigables de depuración y suelen ser inaccesibles después del momento de implementación. Por otra parte, el diseño 
exitoso de algoritmos y protocolos para WSN requiere una vasta experiencia de este tipo complejo de redes distribuidas.

Las simulaciones por computadora han demostrado ser el medio para abordar la tarea desarrollo de algoritmos y protocolos para la ingeniería de WSNs. Con ella se pueden reproducir los efectos del mundo real dentro de un entorno de simulación, incluyendo hasta la propagación de las ondas radiales y los factores ambientales. Así es posible disminuir los esfuerzos operativos y económicos que se generan en una implementación y análisis real.

Ocurre en la mayoría de los casos que debido a la profundidad de análisis efectuado por los simuladores, ocultan el alto número de factores que afectan a la actividad de la WSN como ente único. Puede ocurrir que una simulación durante largo tiempo esté produciendo resultados que nos interesan en absoluto.

La simulación de modelos también puede ser utilizado para el aprendizaje, dado que proporciona soluciones eficaces y libres del costo inherente a la experimentación. La reducción constante del costo de las computadoras permite a las simulaciones convertirse en una técnica muy flexible y de fácil implementación. Hoy, la modelización y la simulación proporcionan un enfoque desarrollado y probado de solución de problemas, evolucionando constantemente a medida que se dispone de mayor potencia de cálculo a un costo menor [171].

\subsection{Desafíos de la simulación}

Para obtener resultados realísticos en una simulación de sistemas inalámbricos se precisa el modelo de las propiedades de tiempo real del nodo, como también aquellos del ambiente y el tipo de radio utilizada. 
Consideremos a un nodo cuya arquitectura de capas se presenta en la ilustración 5.1, y que pretendemos modelarlo con un simulador [172].

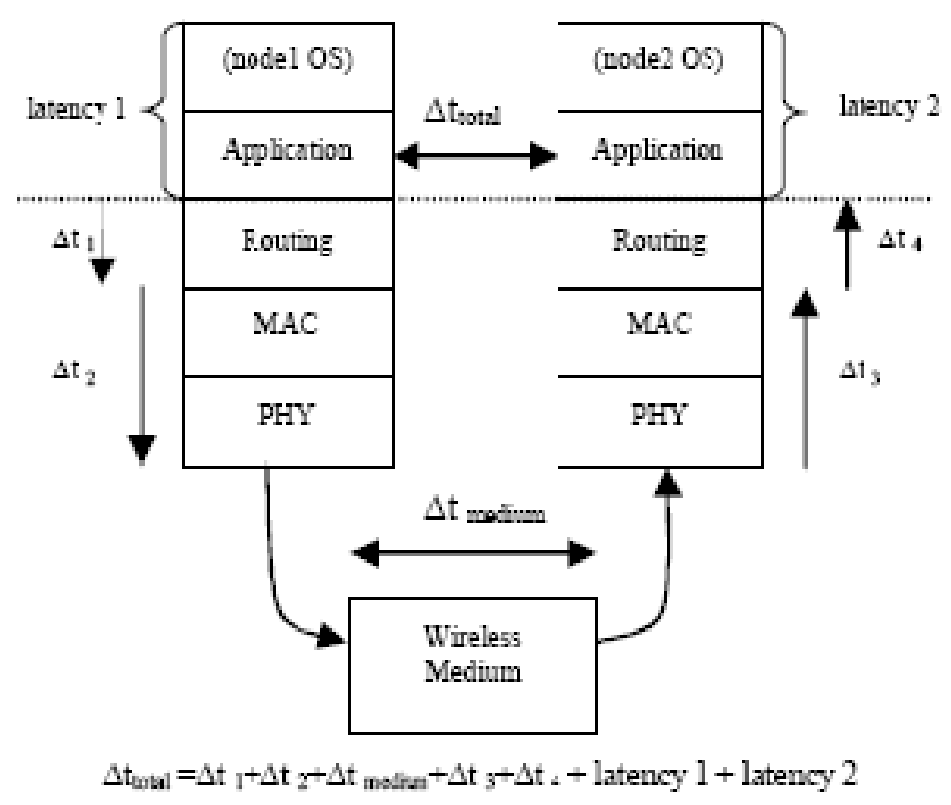

llustración 5.1 Modelo de capas a ser simulado

El parámetro más importante en la ilustración es $\Delta t_{\text {total }}$ dado que indica el tiempo de respuesta de la red. El modelado de las diferentes capas es necesario para evaluar los efectos de los diferentes protocolos y topologías en la operación y retardos en la red.

Considerando el simple ejemplo anterior, el simular a las WSNs es un gran desafío dada la naturaleza de diseño del hardware, limitaciones energéticas, distribución física y número de nodos. Los simuladores se utilizan en la práctica como una manera de evitar recargar de trabajo innecesario a los sensores, o incluso dañarlos; logrando así disminuir considerablemente los tiempos de diseño e incrementar la calidad de la simulación. 
Los simuladores son utilizados para probar algoritmos de redes de sensores inalámbricos a gran escala, testeando principalmente la confiabilidad y robustez. Claro está que estas simulaciones carecen de la precisión de los despliegues del mundo real.

La simulación, permite el diseño, el análisis y la comprensión de redes inalámbricas; evitando implementaciones reales, obteniendo resultados a bajos costos y con mínimos recursos [173], [174]. La implementación de una instalación a escala real de una WSN normalmente es una tarea complicada, casi siempre limitada por cuestiones económicas, que puede reemplazarse casi totalmente con los simulares. Siempre que sea posible se debe realizar un aprovechamiento integral de ambas posibilidades, simulación y testbed.

Tres son los desafíos principales en el estudio las WSN [175]: los métodos analíticos [176], la simulación computada, y el entorno de pruebas.

Los métodos analíticos no están muy desarrollados, de manera que se los considera incapaces de soportar el desarrollo completo de aplicaciones para WSN. Considérese lo complicado que resulta captar todos y cada uno de los detalles en un sistema tan complejo, y en una manera tan formal.

Las experiencias in-situ son una opción siempre atractiva, ya que son el medio eficaz para convencer y demostrar las prestaciones de una aplicación. Sin embargo, debido a las impredecibles influencias ambientales, es muy difícil reproducir los resultados ó aislar las fuentes de errores.

Ya se ha indicado en capítulos anteriores lo laborioso de las tareas de instalación de las WSN, como las tareas que se deben realizar y los problemas conexos a solucionar, que nada tienen que ver con objetivo 
principal de la WSN [84]. Por ello, normalmente los entornos prácticos suelen limitarse a unas pocas docenas de dispositivos [177]. En los escenarios del futuro, se estima que el numero de nodos de las WSN alcanzaran a ser miles, o incluso; millones [178], [179], [180].

\subsection{Ventajas de la simulación}

Las decisiones en un entorno de simulación se pueden comprobar en forma artificial, pudiendo el mismo modelo ser reutilizado innumerables oportunidades. Estos entornos de simulación son mucho más simple de crear, con pocas simplificaciones y utilizando la mas de las veces técnicas analíticas, pudiendo modificar el comportamiento del modelo casi a voluntad. La simulación permite experimentar con variados casos especiales, donde el usuario interactúa con la herramienta, posibilitando el análisis de esas interacciones.

La ventaja fundamental de la utilización de simulaciones en las WSN es en el campo económico, donde con poca inversión inicial se obtiene resultados rápidamente; permitiendo con ello que se pueda mejorar la investigación y el desarrollo sobre el tema.

Existen diferentes métodos para modelar los hechos del mundo real, hechos en las que las entidades cambian debido a la ocurrencia de eventos particulares, y el modelo de evolución depende de las interacciones de tales eventos y su posición temporal. Este tipo de entidades, que se representan con variables observables en tiempo real (modelado dinámico de sistemas), se denominan sistemas de eventos discretos. Frente a la simulación de tiempo continuo, la simulación de eventos discretos se caracteriza por un control en la variable del tiempo que permite avanzar a intervalos variables, en función de la planificación de ocurrencia de tales eventos a un tiempo futuro. Un requisito para aplicar esta técnica es que las variables que definen el sistema no 
cambien su comportamiento durante el intervalo simulado. Los sistemas de eventos discretos dinámicos son naturalmente simultáneos y no lineales. Como puede apreciarse, es muy complicado tratar de encontrar las soluciones analíticas generales.

La modelización y simulación de eventos discretos supone que, aunque el tiempo es contínuo, sólo un número finito de eventos pueden ocurrir en un período determinado. Por ello un simulador de eventos discretos, puede ser muy eficaz, ya que sólo necesitamos representar un cambio de estado cuando se verifique la ocurrencia de un evento.

\subsection{Datos a simular}

Con las simulaciones se pueden obtener datos empíricos de parámetros tales como uso de CPU, uso de la memoria, rendimiento; como también realizar estimaciones de consumos de energía del nodo. El objetivo primordial de la herramienta es medir el impacto de cambios en parámetros configurables de la red, el rendimiento de la misma y la estimación del consumo de potencia.

Tan importante como obtener datos empíricos, es realizar ciertas pruebas en escenarios distintos al real; para así constatar si alguna modificación en el nodo puede optimizar otros recursos del mismo. Como ejemplo, se podría modificar la arquitectura de una WSN antes de construirla en la plataforma real. Así, toda nueva implementación de nuevos protocolos MAC, se pueden implementar, depurar y probar en un simulador; obteniendo valores de consumo de potencia y de funcionamiento antes de implementarlo físicamente en el nodo.

La simulación permite aislar ciertas zonas o áreas de la red, o establecer ciertos parámetros prácticos. Permite presuponer hechos ideales, totalmente distintos a los encontrados en la realidad como que las 
colisiones de paquetes, la interferencia y el ruido no se producen en la red, que los nodos están siempre perfectamente sincronizados entre sí, o que determinados nodos no consumen energía. Estas simplificaciones, permiten una evaluación más rápida y fácil del proceso de comunicación en la red, pero pueden dar lugar a algoritmos que no pueden trasladarse a la práctica. Toda simulación es tan realista como lo son los modelos y las hipótesis en que se basa.

Por la estructura propia de las WSN los aspectos susceptibles de simulación son a) los nodos y su hardware, b) comportamiento y protocolos asociados a la red y c) el software utilizado, tanto a nivel de aplicación como de sistema operativo.

\subsection{Simulación en WSN}

Mientras que las herramientas de simulación ha avanzado razonablemente en el campo de las MANET, la simulación de WSN requiere de la implementación de otros factores importantes como el canal de radiofrecuencia, el entorno físico determinado y modelos precisos de utilización energética.

Es tarea del simulador, presentar un modelo y predecir el comportamiento en un ambiente real ante diferentes escenarios probables. Estos enfoques basados en simulaciones, como se ha dicho anteriormente, son ventajosos en costos, escalabilidad, tiempo y facilidad de implementación.

Los simuladores actuales utilizados en WSN están categorizados en los que se han desarrollado como una extensión a los simuladores de red existente, como SensorSim [181], que nace como una extensión del ns-2 [182]; y los que han sido diseñados específicamente para la simulación de redes de sensores como J-Sim [183] y Shawn [184]. 


\subsection{Simuladores comunes en WSN}

Las herramientas de simulación se han desarrollado apuntando a características inherentes de las WSN, donde cada uno de ellas ofrece sus mejores resultados. La tabla 5.1 presenta a los simuladores más conocidos.

Tabla 5.1 Simuladores típicos en WSN

\begin{tabular}{|c|c|c|c|c|c|c|}
\hline Simulador & Disponibilidad & Escalabilidad & $\begin{array}{c}\text { Lenguaje } \\
\text { Programación }\end{array}$ & $\begin{array}{c}\text { modelo } \\
\text { sensores }\end{array}$ & $\begin{array}{c}\text { modelo } \\
\text { inalam. }\end{array}$ & $\begin{array}{c}\text { Evento/ } \\
\text { Tiempo }\end{array}$ \\
\hline NS2 & $\bullet$ & $\bullet$ & $\mathrm{C}++$ & $\mathrm{X}$ & $\mathrm{X}$ & Evento \\
\hline GLOMOSIN & $\bullet$ & $\bullet$ & $\mathrm{C}++$ & $\mathrm{X}$ & $\mathrm{X}$ & Evento \\
\hline OPNET & $\mathrm{X}$ & $\bullet$ & $\mathrm{C}++$ & $\mathrm{X}$ & $\mathrm{X}$ & Evento \\
\hline SensorSim & $\bullet$ & $\bullet$ & $\mathrm{C}++$ & $\bullet$ & $\bullet$ & Evento \\
\hline Shawn & $\bullet$ & $\bullet$ & $\mathrm{C}++$ & $\mathrm{X}$ & $\bullet$ & Evento \\
\hline Tossim & $\bullet$ & $\mathrm{X}$ & $\mathrm{nesC}$ & $\bullet$ & $\bullet$ & Evento \\
\hline EmStar & $\bullet$ & $\bullet$ & nesC/C++ & $\bullet$ & $\bullet$ & $\begin{array}{c}\text { Evento } \\
\text { Tiempo }\end{array}$ \\
\hline SENS & $\bullet$ & $\bullet$ & $\mathrm{C}++$ & $\bullet$ & $\bullet$ & Evento \\
\hline MANTIS & $\bullet$ & $\mathrm{X}$ & $\mathrm{C}$ & $\bullet$ & $\bullet$ & Tiempo \\
\hline SIESTA & $\bullet$ & $\bullet$ & Java & $\bullet$ & $\bullet$ & Tiempo \\
\hline PROWLER & $\bullet$ & $\bullet$ & MatLab & $\mathrm{X}$ & $\mathrm{X}$ & Evento \\
\hline JPROWLER & $\bullet$ & $\bullet$ & Java & $\mathrm{X}$ & $\mathrm{X}$ & Evento \\
\hline
\end{tabular}

En la ilustración 5.2 se presentan las herramientas de simulación que con más frecuencia son utilizadas en investigación de WSN. Se presentan las mismas a lo largo de dos de dos ejes: el nivel de abstracción y el tamaño típico de red que es capaz de simular. Se debe considerar que esto no expresa el tamaño máximo posible de la red, sino que refleja los dominios típicos de aplicación. 


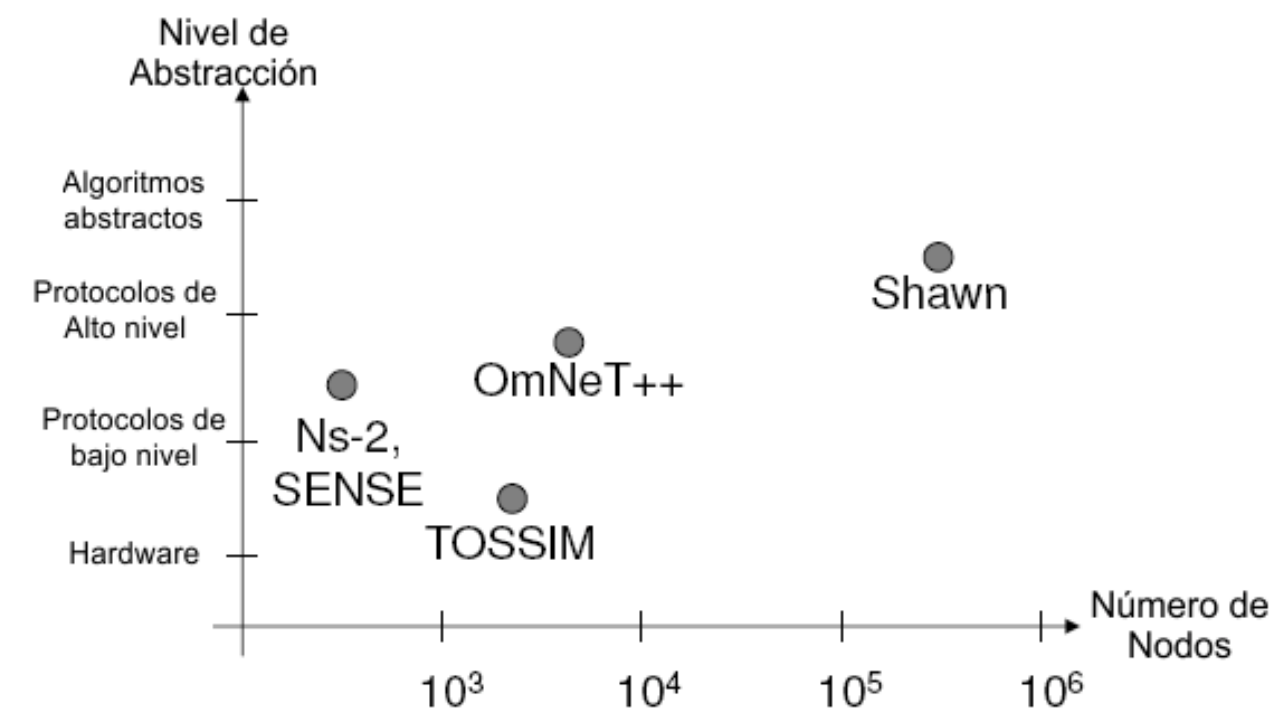

Ilustración 5.2 Ámbito de aplicación de distintos simuladores WSN

\subsubsection{Network Simulator 2 (NS-2)}

NS-2 es un simulador de eventos discretos dirigidos a la creación de redes destinadas a la investigación, siendo considerado como uno de simuladores más importantes utilizados en la investigación de las WSN [185]. Proporciona un apoyo sustancial para la simulación de TCP, ruteo y protocolos de multidifusión a través de redes y conexiones inalámbricas locales y remotas [182].

NS-2 se centra en la simulación de las capas ISO/OSI; como también en el consumo de energía y de los fenómenos en la capa física. Incluye un amplio depósito de protocolos, generadores de tráfico y herramientas para simular TCP y protocolos de enrutamiento multicast a través de redes cableadas e inalámbricas, y hace unos pocos años fue el simulador preferido de los investigadores de MANET [185]. Cuenta con simulación de seguimiento detallado e incluye la herramienta de visualización NAM ${ }^{1}$

\footnotetext{
${ }^{1}$ NAM: Network Animator
} 
para poder reproducir el tráfico observado en la red en el momento que se preestablezca.

Si bien se han reportado en algunos trabajos dificultad de modificar código, y/ó desarrollar extensiones para NS-2 [186]; existen extensiones para las simulaciones de WSN, la que incluyen modelos de sensores, de baterías, pilas de protocolos y herramientas de generación de escenarios [187], [181]. NS-2 posee una escalabilidad limitada, principalmente debido a que no se logra la relación armónica entre los objetos y el sistema orientado a objetos. También influye la inexistencia de un modelo de aplicación. El desarrollo de una extensión denominada SensorSim [181] ha mejorado en todo sentido los puntos anteriores.

El alto nivel de detalle de las simulaciones, a nivel de intercambio de paquetes, consume mucho tiempo de procesamiento, dado que existe una relación directa entre tiempo de ejecución y cantidad de paquetes a intercambiar. NS-2 es capaz de simular hasta 16.000 nodos. Debido al elevado consumo de recursos ha sido demostrada la impracticabilidad de obtener un buen rendimiento con este simulador en condiciones de trabajo con un número de nodos mayor al millar.

\subsubsection{SensorSim}

SensorSim [188] ha sido desarrollado tomando como base a NS-2, tratando de evolucionar en lo referente a simuladores de WSN. Es capaz de simular canales de comunicación, modelos de batería y sensores, pilas de protocolos para sensores, generación de escenarios y simulación híbrida. Sin embargo la herramienta está aun en desarrollo y nunca fue finalizada debido a la imposibilidad de obtener el nivel de soporte requerido. 


\subsubsection{Prowler and JProwler}

Probabilistic Wireless Network Simulator- Prowler- [189] es un simulador WSN de eventos discretos, desarrollado para simular nodos MICA con sistemas operativos TinyOS, como también WSN genéricas. Puede operar en modo determinístico ó probabilístico. Es capaz de simular cambios dinámicos en el ambiente, con un número de sensores capaces de correr diferentes aplicaciones. Dado que utiliza como plataforma a MATLAB, es una herramienta ideal para el desarrollo de prototipos, poseyendo una excelente capacidad de visualización. JProwler [190] es una herramienta idéntica a Prowler, pero desarrollada en Java.

\subsubsection{OMNeT++}

Objective Modular Network Testbed in C++ (OMNeT++) [191] es un simulador modular de eventos discretos. Es similar a NS-2 y apunta también al modelo ISO/OSI de capas. Capaz de simular entornos de pocos miles de nodos, y presentando gráficamente a la red y el flujo de datos.

OMNeT++ se articula en torno a un sistema modular donde los módulos simples, similar a la pila de protocolos; contienen algoritmos, representando al nivel más bajo de la jerarquía. Los módulos compuestos, tal como un nodo sensor; contienen los módulos simples que interactúan unos con otros por medio de diferentes mensajes.

El simulador está escrito en $\mathrm{C}++$ y es provisto con lenguaje de configuración propio llamado NED.

\subsubsection{GTSNetS}

Este simulador es una extensión del Georgia Tech Network Simulator (GTNetS) [192], utilizando al máximo sus opciones de diseño para la 
obtención de un rendimiento óptimo. Incorpora los modelos de las diferentes unidades funcionales de un nodo sensor, caracterizando el consumo de energía de cada uno de ellos. En particular, ha sido capaz de simular redes de sensores de varios cientos de miles de nodos utilizando menos de 2 GB de memoria.

\section{$5.5 .6 \mathrm{~J}-\mathrm{Sim}$}

A fin de superar los problemas de escalabilidad inherente a las estructuras orientadas a objetos, se ha diseñado J-Sim [193], [194] en torno un esquema orientado a componentes. Si bien esto aumenta su escalabilidad, la elección de Java reduce la eficiencia del simulador mientras que lo hace verdaderamente multiplataforma. J-Sim es relativamente complicado y, debido a la inexistencia de usuarios reales, no ha tenido demasiada aceptación.

\subsubsection{SENS}

Sensor, Environment and Network Simulator [195] es un simulador a nivel de aplicación conformado por cuatro componentes extensibles e intercambiables: aplicación, red, capa física y ambiente. Éste último es el más sofisticado de los componentes incluyendo modelos de fenómenos físicos y distribución. SENS permite la portabilidad de la aplicación ejecutable, dado que ejecuta el mismo código en la simulación que en el ambiente real. Lamentablemente detalles sobre los protocolos de ruteo, módulos de emulación y visualización son desconocidos, los HOWTO o manuales no se encuentran disponibles.

\subsubsection{SENSE}

SENSE (Sensor Network Simulator and Emulator) [186] mejora la eficiencia de J-SIM, proporcionando una arquitectura de componentes 
orientada a la programación en $\mathrm{C}++$. Con esto tiende a la mejora de la eficiencia en la comunicación, defecto de J-Sim. Los autores mencionan la portabilidad y la reutilización como factores clave en SENSE. Ofrece diferentes modelos de batería, redes simples y capas de aplicación; además de una implementación de aplicaciones IEEE 802.11 [38]. En su versión actual, proporciona un motor de simulación secuencial que puede simular alrededor de 5.000 nodos.

\subsubsection{TOSSIM}

TOSSIM (The TinyOS mote simulator) [196] es un simulador y emulador de redes inalámbricas de sensores, simulando el código TinyOS [60], [197], [198], [199] para los Nodos MICA [7], [8]. TOSSIM ofrece ventajas evidentes en aquellos proyectos que utilizarán los nodos mencionados, convirtiéndose en el simulador específico para la plataforma. Compila el código TinyOS, y el ejecutable obtenido puede correrse en una PC común. Posee una GUI, TinyViz, donde se puede observar e interactuar con las simulaciones en curso. Para la modelación del consumo energético del ambiente en estudio se ha desarrollado la extensión PowerTOSSIM [200], para modelar y simular el consumo energético de los nodos Mica2 [7].

\subsubsection{Shawn}

La virtud de Shawn [174] tiene que ver con el hecho que la herramienta simula los efectos de un fenómeno y no al fenómeno en sí. Para ello reemplaza los efectos de bajo nivel, con modelos intercambiables y abstractos. Shawn permite seleccionar la granularidad de la simulación, adaptando su comportamiento a las necesidades específicas. 


\subsection{Selección del simulador}

De todas herramientas que se han presentado anteriormente, cada una de ellas posee un rendimiento excelente en determinado dominios específicos. Lamentablemente parece ser que ninguna de estas permite el escalamiento de protocolos de alto nivel y de algoritmos abstractos para el manejo de grandes redes. Este escalón importante es cubierto por Shawn [174], [201], [184].

La diferencia sustancial entre Shawn y los otros simuladores, tiene que ver con el enfoque de desarrollo de la herramienta. No compite con los otros simuladores en sus tareas de simular redes, sino que se realiza la esa tarea en base algorítmica. La herramienta posee capacidad de soporte y escalabilidad para redes muy numerosas. Con Shawn es posible seleccionar libremente el modelo de implementación.

\subsubsection{Simulación de efectos}

Mientras que Shawn simula los efectos, los demás simuladores realizan un análisis completo de la capa MAC, incluyendo las propiedades de propagación radial afectadas por la atenuación, colisiones y propagación multipath. Shawn solamente modela los efectos de la capa MAC en la aplicación, por ejemplo pérdida de paquetes, corrupción de datos, retardo, etc. Los escenarios planteados por Shawn son más predecibles, y poseen la cualidad de ser implementados en la práctica eficientemente. Debe dejarse en claro no es capaz representar en el mismo nivel de detalle de la capa física, ni de los fenómenos a nivel de paquetes; como lo realiza NS-2.

La interpretación de los resultados de una simulación debe, ineludiblemente, considerar las características y propiedades de los modelos individuales involucrados. 


\subsubsection{Escalabilidad}

Shawn presenta aquí ventajas relativas a otros simuladores [74]. Al simplificar la estructura de varios parámetros de bajo nivel, los cálculos, que son los principales consumidores de ciclos de CPU, son reemplazados por substitutos más rápidos siempre que estos no afecten a las propiedades del evento. Este paradigma permite simular vastas redes, mediante la administración de diversos escenarios, a los cuales Shawn puede ser configurado específicamente.

\subsubsection{Elección libre del modelo}

Shawn soporta un ciclo de desarrollo multietapas, mediante el cual los investigadores pueden elegir libremente el modelo de implementación como se indica en la ilustración 5.3 [74] .

Utilizando Shawn, no existe el límite superior en cuanto a la implementación de protocolos distribuidos. El razonamiento de este enfoque sería el siguiente: teniendo una primera idea de un nuevo algoritmo, la etapa naturalmente posterior no es diseñar un protocolo totalmente distribuido, sino realizar un análisis estructural del problema considerado. Para comprender mejor el problema en esta fase, puede ayudar el tomar algunas redes como ejemplo, para así analizar su estructura y la representación gráfica para la misma.

La próxima etapa sería implementar una versión centralizada del algoritmo para alcanzar rápidamente una versión prototipo. Un algoritmo central posee acceso total a todos los nodos, poseyendo asimismo una vista plana y global de la red. Con esto se obtiene una primera impresión del rendimiento del algoritmo considerado. La retroalimentación produce una mejora en el rendimiento del algoritmo. Una vez que se obtiene un 
buen servicio centralizado, se avanza con la posibilidad de la implementación distribuida.

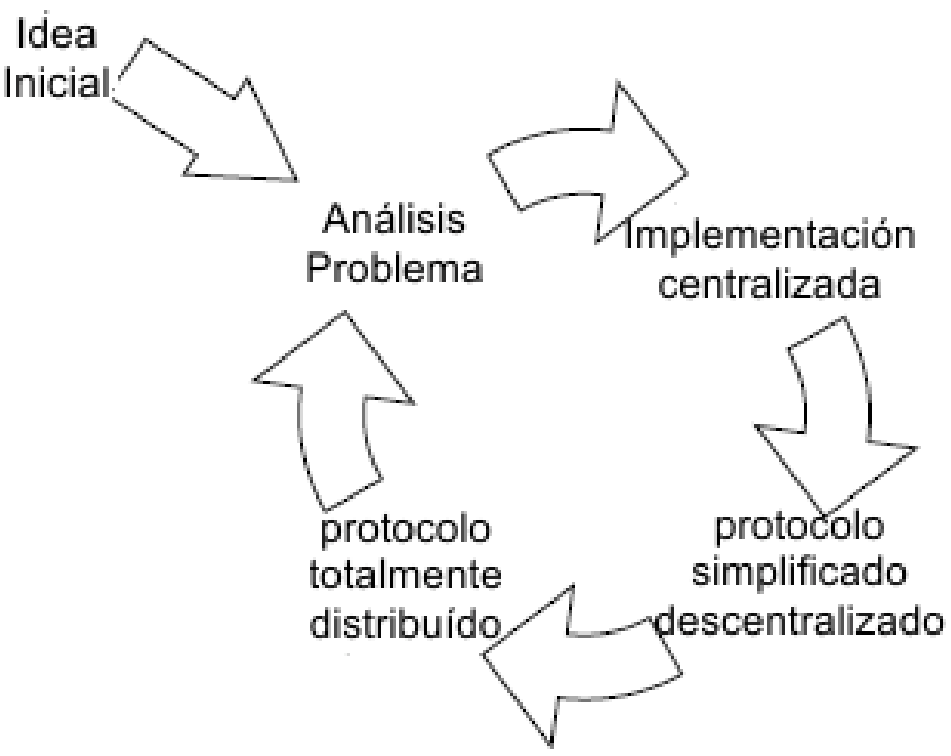

Ilustración 5.3. Ciclo de desarrollo posibilitado por Shawn

Esta versión simplificada puede ser transformada en un protocolo que intercambie mensajes de red conteniendo carga útil. Entonces es posible investigar lo referente al número de mensajes, consumo de energía, tiempo de ejecución, capacidad de evitar perdida de mensajes y los efectos del medio ambiente. Así se completa el ciclo.

\subsubsection{Arquitectura Shawn}

La arquitectura de Shawn comprende tres elementos fundamentales, las que se indican en la ilustración 5.4 [184]: modelos, secuenciador y ambiente de simulación. Resaltando la escalabilidad y la flexibilidad de 
esta herramienta, cada uno de estos aspectos es influenciado por uno o más de los modelos.

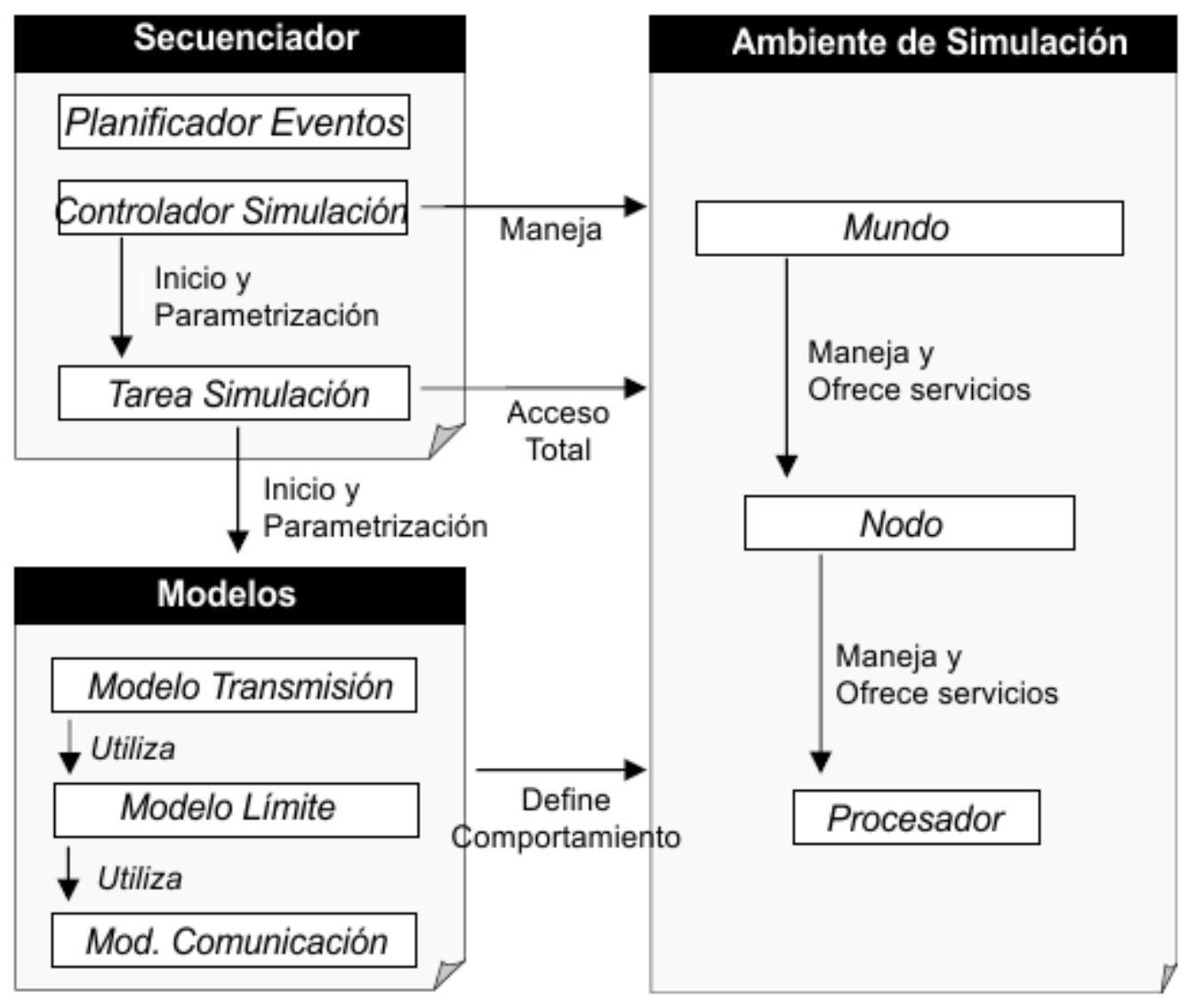

llustración 5.4. Arquitectura de alto nivel de Shawn

El secuenciador es la unidad central de coordinación de la herramienta, donde se configura la simulación, ejecutando cada una de las tareas secuencialmente; realizando también el control del proceso. El ambiente de simulación se corresponde con el mundo virtual donde los nodos sensores realizan sus tareas. 


\subsubsection{Modelos}

\subsubsection{Modelo de Comunicación}

Cuando un determinado sensor que está siendo simulado por Shawn transmite un mensaje, los receptores potenciales de este mensaje deben ser identificados por el simulador. Con ello se reconoce quienes son las entidades que podrán establecer comunicación entre sí. Esta tarea la realiza el modelo de comunicación, cuya interfaz en código $\mathrm{C}++$ se esquematiza en la ilustración 5.5; donde se invoca un método simple para determinar si el nodo b es alcanzable por el nodo a [202].

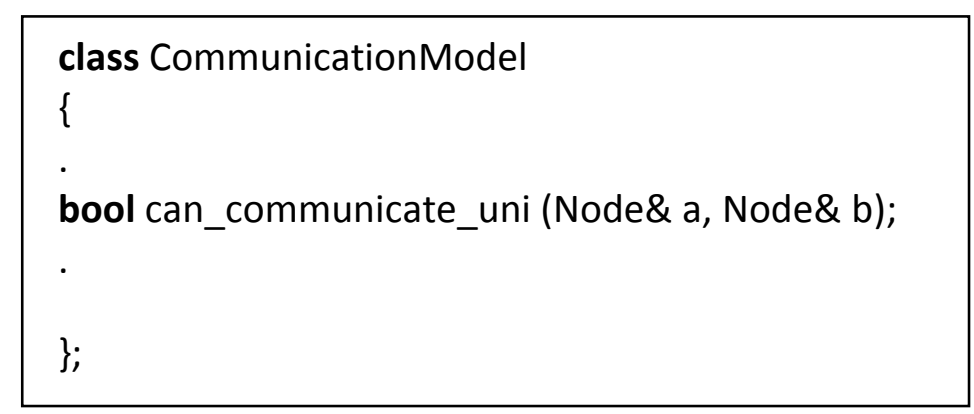

Ilustración 5.5. Extracto de la Interfaz del Modelo de Comunicación de Shawn

Cuando se aplica la interfaz anterior, puede utilizarse uno de los diferentes modelos de comunicación provistos por Shawn, de acuerdo a los indicados en la llustración 5.6. 


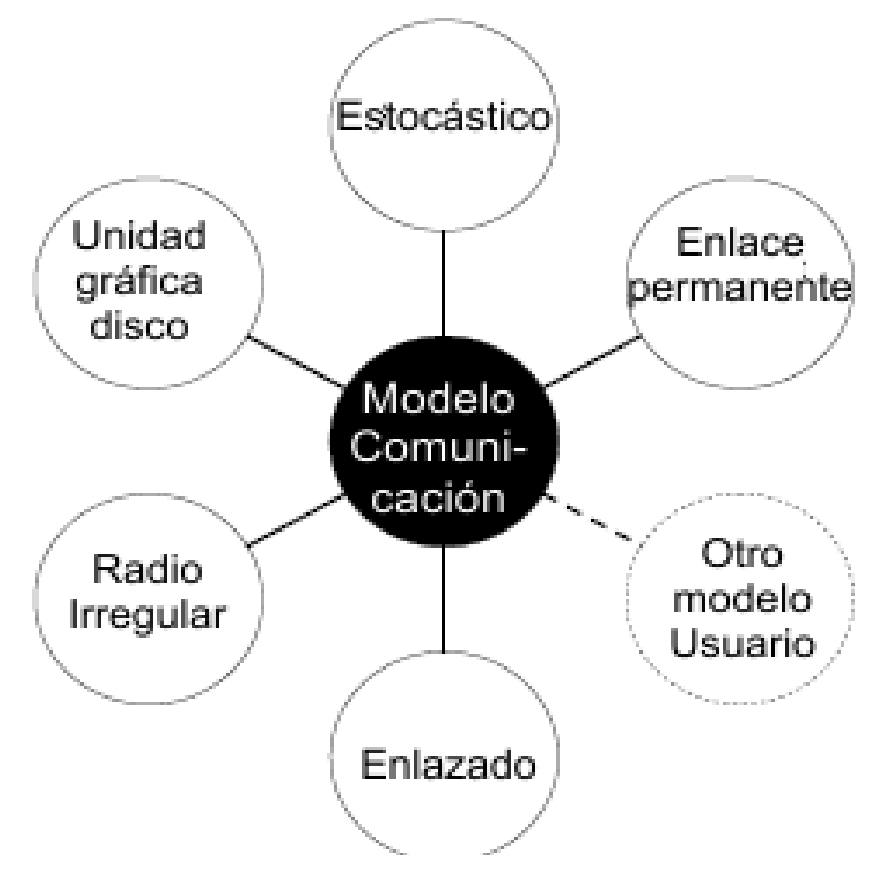

Ilustración 5.6. Vista de los modelos de comunicación de Shawn

De los cinco modelos de comunicación presentados, los más frecuentemente utilizados en investigaciones en WSN son solamente tres. El modelo de radio UDG (Unit Disk Graph), el más utilizado en el ámbito de investigaciones por su simplicidad, considera que la potencia de la señal de radio varía con el cuadrado de la distancia desde el nodo emisor. Dada la potencia mínima de señal requerida para recepción, dos nodos podrán comunicarse en forma bidireccional si la distancia entre ellos es menor que $\boldsymbol{r}_{\text {max. }}$ En la ilustración 5.6 se muestran los nodos compartidos (puntos rellenos) por dos nodos considerados en determinado estudio, n1 y n2 (con doble circulo). La intersección de ambos círculos (UDG) encierra a los nodos que podrán comunicarse con n1 y n2. 


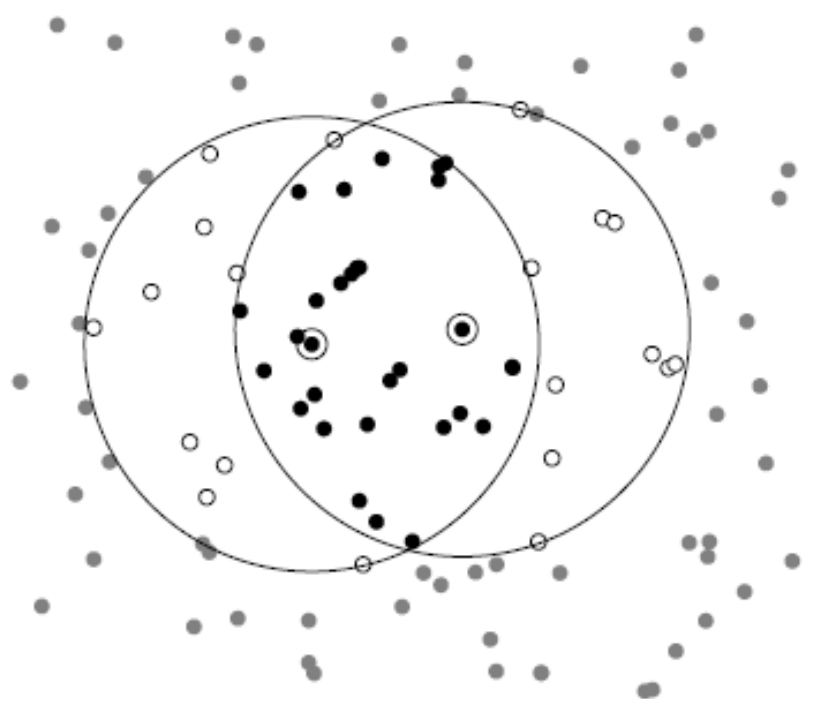

Ilustración 5.7. Modelo radial UDG

En el modelo de irregularidad radial está basado en el comportamiento en la vida real, proponiendo una dependencia angular del alcance, la que varía entre las distancias máxima y mínima [203], [204]. Dicho factor determina el máximo alcance de acuerdo al ángulo de cada vínculo y a las características del terreno donde se implemente la simulación, ver Ilustración 5.7. A diferencia de UDG, el modelo de irregularidad radial (RIM) calcula también rendimientos de enlaces unidireccionales.

El modelo enlace permanente permite la especificación de enlaces estáticos para pre-definir las relaciones de comunicación, en situaciones tales como cuando se enlaza por cable a los nodos sink. 


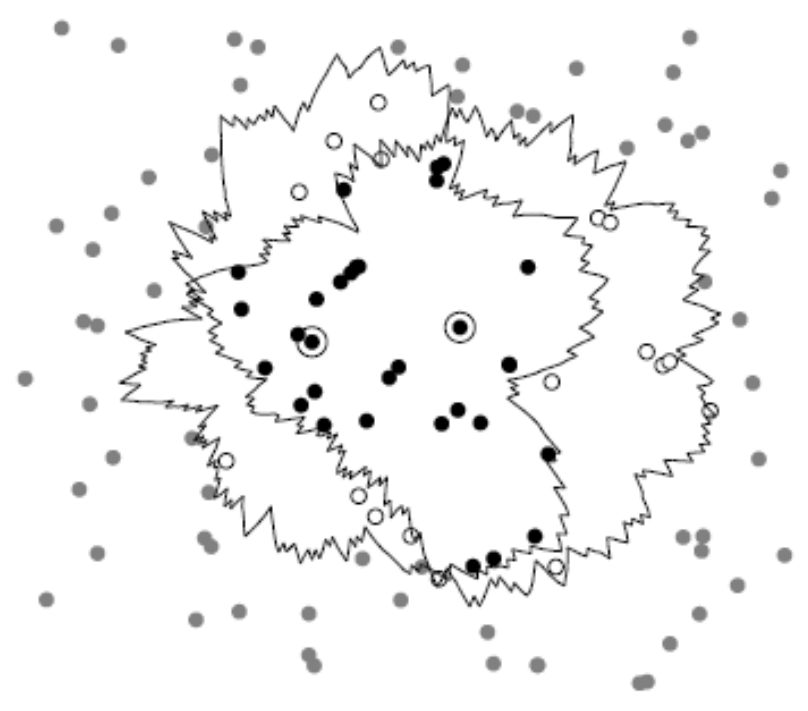

Ilustración 5.8. Modelo Radial RIM

El modelo enlazado permite compatibilizar y armonizar múltiples modelos de comunicación en uno solo. Como ejemplo, la mayoría de los nodos sensores utilizan el modelo de transmisión UDG, mientras que los nodos gateways a redes mayores mantienen conexiones cableadas modeladas como enlaces permanentes.

\subsubsection{Modelo límite ó borde}

Este modelo permite representar gráficamente a la red. Los nodos simulados son los vértices del gráfico y se añade un borde entre dos nodos, siempre que el modelo de comunicación se valide. Para el montaje de esta representación gráfica, el modelo de bordes repetidamente consulta el modelo de comunicación. Por lo tanto, es posible tener acceso a los vecinos directos de un nodo, los vecinos de los vecinos, y así sucesivamente. Esto es utilizado por Shawn para determinar los posibles 
receptores de un mensaje por iteraciones sucesivas del modelo con vecinos del nodo emisor.

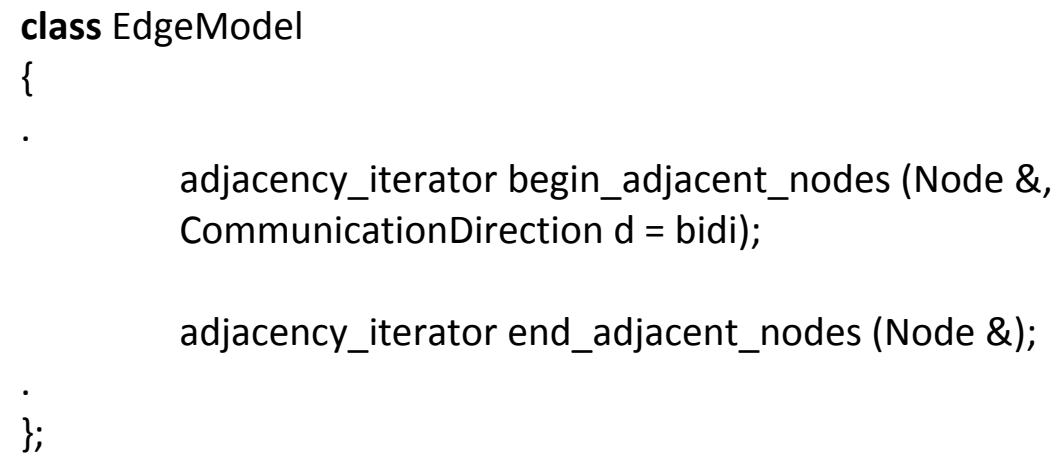

Ilustración 5.9 Código de Interfaz aplicación del modelo de borde en Shawn

En la ilustración 5.9 se puede observar la interfaz $\mathrm{C}++$ que se utiliza para las implementaciones del modelo de borde ó límite. En esencia, son dos los métodos que proporcionan la capacidad de iterar contra los vecinos de un nodo hacia una dirección específica de comunicación. El parámetro de dirección de la comunicación define la forma en la que será considerada la propiedad "vecino" y puede ser uno de los siguientes: in, out, bidirectional or any.

De acuerdo a las necesidades de la simulación planteada, Shawn posibilita una serie de opciones para seleccionar el modelo de límite o borde (ilustración 5.10). 


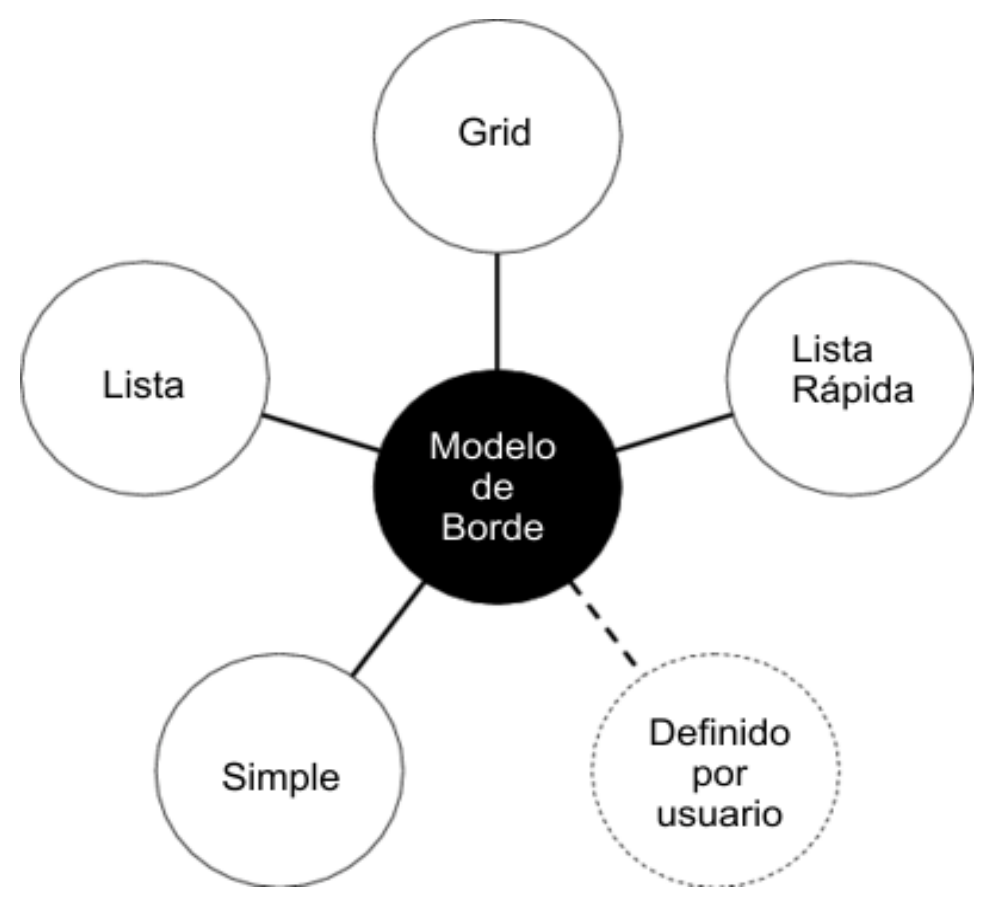

llustración 5.10 Modelos de borde de Shawn

\subsubsection{Modelo de transmisión}

Cuando un nodo transmite un mensaje, el comportamiento del canal de transmisión no necesariamente será idéntico al observado durante la transmisión del mensaje inmediatamente anterior. Puede existir algún bloqueo por tráfico cruzado desde otros nodos, debido a lo cual se puede ir degradando continuamente la calidad del canal de comunicación por interferencia mutua. Shawn, en sus modelos de transmisión, es capaz de modificar las propiedades de la transmisión de un mensaje individual, retrasando, alterando o eliminando mensajes. Por lo anterior, un mensaje puede no arribar a su destino, aún si el modelo de comunicación indica que los dos nodos pueden comunicarse, y que el modelo de borde haya listado a ambos nodos como vecinos. 
En la ilustración 5.11 se indican los modelos de transmisión provistos por Shawn, cubriendo tanto las implementaciones abstractas, como aquellas cercanas a la realidad.

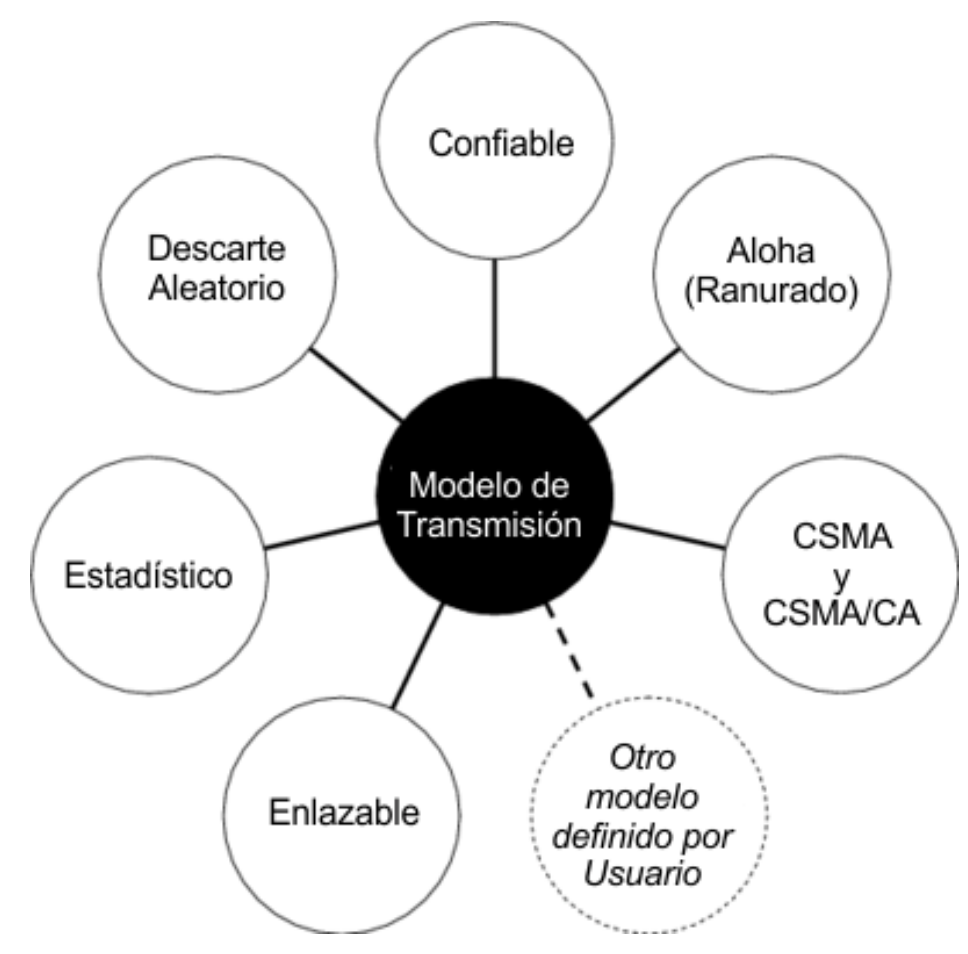

Ilustración 5.11. Modelos de transmisión provistos por Shawn

El modelo de transmisión confiable despacha los mensajes inmediatamente. El de descarte aleatorio es similar, descartando mensajes de una probabilidad dada, pero nunca retarda o altera los mensajes. La implementación estadística no envía dato alguno, sino graba información como conteo de paquetes, diferentes tipos de mensajes, etc. El enlazable permite que el modelo de transmisión procese un mensaje secuencialmente, con lo que un mensaje puede ser contado, luego demorado o descartado combinando los modelos indicados 
anteriormente. En el mismo gráfico se muestran dos implementaciones adicionales que indican un mayor grado de acercamiento a la realidad que los mencionados anteriormente. Estas simulan los bien conocidos y estudiados casos de los esquemas de Acceso al medio CSMA/CA y ALOHA ranurado [205], [165], [206].

\subsubsection{Secuenciador}

El secuenciador de Shawn es el centro de control de la simulación, preparando el mundo en el cual los nodos simulados realizaran sus tareas. Crea las instancias y parametriza las implementaciones de los modelos, controlando así también la simulación. El secuenciador realiza, según la ilustración 5.3 las siguientes tareas: simulación, control de la simulación y programador de eventos.

\subsubsection{Tareas de simulación}

Las tareas de simulación son piezas de código que se invocan desde la configuración de la simulación y son suministrados por el usuario. No están directamente relacionados con la aplicación simulada pero tienen acceso a todo el entorno de simulación y por lo tanto son capaces de realizar una amplia gama de tareas. Ejemplos de su aplicación son la gestión de simulaciones, la recopilación de datos de los nodos individuales o correr algoritmos centralizados.

\subsubsection{Controlador de Simulación}

Actúa como repositorio central para todas las implementaciones del modelo, ejecutando la simulación mediante transformación de los parámetros de entrada en las invocaciones desde las tareas de simulación. De esta manera, oficia de intermediario entre el núcleo de simulación de Shawn y el usuario. La aplicación, por defecto, lee la 
configuración de comandos de un archivo de texto o de una secuencia de entrada estándar

\subsubsection{Planificador de eventos}

Shawn utiliza un planificador de eventos discretos como modelo de tiempo, el cual realiza el cronometraje. Los objetos que precisan tener nociones de tiempo se deben registrar con el planificador de eventos.

En el momento que la simulación pasa al evento siguiente, el planificador efectúa la notificación a los nodos registrados. Este proceso continúa hasta que todos los nodos se hayan apagado ó hasta que haya transcurrido el tiempo máximo configurado.

\subsubsection{Ambiente de Simulación}

El entorno de simulación es el hogar del mundo virtual en el que residen los objetos de simulación. Los nodos a su vez sirven como contenedores para los llamados "procesadores". Los desarrolladores aplican su lógica de programación como instancias de estos procesadores. Por disociación de la aplicación dentro de un procesador de un nodo, pueden ser fácilmente combinados en una sola simulación múltiples aplicaciones de ejecución sin necesidad de cambiar sus implementaciones. Por ejemplo, un procesador puede ejecutar un protocolo de aplicación específica, mientras que otro recoge los datos estadísticos.

\subsection{Resumen}

Hoy en día, existen una gran cantidad de herramientas para simular redes de sensores inalámbricos. Se hace hincapié en el capítulo que cuando mayor es la precisión requerida de la simulación en cuanto a la calidad de datos obtenidos, tanto mayor es el tiempo de corrida experimental. 
Se realiza una presentación y análisis de los diferentes simuladores que se utilizan actualmente en el ámbito de las WSN. Se presentan los desafíos de la simulación, las ventajas de adoptarla y los beneficios de correr un simulador como primera medida en los proyectos de implementación práctica de las redes de sensores.

En el capítulo se ha selecciona a Shawn como simulador para la tesis, dado que substituye los efectos de bajo nivel con modelos abstractos e intercambiables, de manera que la simulación puede ser utilizada para redes de gran número de nodos, en un plazo razonable, manteniendo el foco de la investigación en el problema central. 
Capítulo 6 : Experiencia y

Resultados Obtenidos 
I método científico estándar indica que para entender a las
situaciones más complicadas, es menester analizarlas de una
manera jerárquica, esto es organizadamente desde arriba hacia abajo. Este método es válido para la organización de una gran cantidad de estructuras nuevas; por lo que muchos aspectos de nuestra vida diaria han permitido un análisis jerárquico y centralizado con el empleo de computadoras. El resultado de dicho análisis indica que es imposible ignorar que una gran variedad de sistemas poseen estructuras muy diferentes; por ejemplo la estabilidad de nuestra moderna estructura social, económica y política se basa en el hecho que están basados fundamentalmente en mecanismos descentralizados, distribuidos y auto organizados. Internet es una de estas historias exitosas donde una visión tecnológica ha tenido un impacto tremendo en muchísimos aspectos de nuestras vidas.

Hoy se está experimentando en las TICs los efectos del vasto número de objetos heterogéneos existentes en una red descentralizada; particularmente los efectos de la escala en la interacción entre hardware, software, algoritmos y datos, en donde la mayoría de los fenómenos ocurren sorpresivamente y no como una definición del diseño.

Las WSN son una de las tecnologías fundamentales en la Internet del futuro, y representa la expansión de Internet al mundo real, permitiendo acceder a indicadores que pueden ser consultados en tiempo real. Estos sensores son fuentes de información tan variadas como lo son las medidas que realizan. Los hay de temperatura, de luminosidad, de presión, de humedad, de velocidad, de aceleración, de presencia, de volumen y un sin fin de magnitudes que se nos ocurran. Si a estos sensores que nos reportan información valiosa para nuestras actividades, les añadimos la capacidad de comunicación inalámbrica y la posibilidad de formación de redes ad hoc, estamos en presencia de una WSN. 


\subsection{Marco de la Experiencia}

En junio de 2008 instituciones académicas y de investigación europeas, en un esfuerzo conjunto, pusieron en marcha el proyecto WISEBED. Este consorcio forma parte de la "Estrategia Europea para Internet del Futuro" respaldada fuertemente por la Sociedad Alemana de Informática. El objetivo del WISEBED es proveer una infraestructura de interconexión de redes de sensores inalámbricos a gran escala con fines de investigación, persiguiendo un enfoque interdisciplinario.

Los nueve participantes son instituciones académicas y de investigación europeas, a saber: las Universidades de Lübeck, Berlín y Braunschweig de Alemania, el Research Academic Computer Technology Institute de Grecia, la Universidad Politécnica de Cataluña de España, las Universidades de Berna y Ginebra de Suiza, Universidad de Delft de Holanda, y la Universidad de Lancaster en el Reino Unido de Gran Bretaña.

En este contexto las Universidades Nacionales de La Plata y Misiones; de la República Argentina, y la Universidad de Lübeck; en la República Federal de Alemania, han generado un proyecto conjunto cuyo objetivo evolucionar hacia una red global de sensores, instalando una red de sensores inalámbricos en la República Argentina.

Esta red pretende interconectarse con emprendimientos similares en Europa, permitiendo acceder a los datos recogidos en ambientes naturales de nuestro país. Cabe aclarar, que la red propuesta en nuestro país será la primera extra paneuropea en intervenir en estos proyectos.

\subsection{Metas}

Las WSN son cada vez más populares en el campo del monitoreo ambiental debido a sus propiedades y capacidades potenciales. Sin 
embargo, pocas de estas actuales aplicaciones consisten de implementaciones donde los datos sean trabajados en alguna medida dentro de la red, sino que son simplemente terminales de medición.

Se ha establecido como meta establecer una red piloto de sensores e implementar un vínculo a la red de redes, para avanzar luego mancomunadamente sobre cada una de las limitaciones.

Se ha adoptado el hardware de red orientado a la aplicación, y la plataforma del sistema para la adquisición de datos, validación, procesamiento y visualización se presenta sistemáticamente. Se han considerado varias estrategias de manera de garantizar la utilidad de la información generada por la implementación práctica. Parte importante de las tareas desarrolladas ha tenido que ver con la integración de los visualizadores de los eventos en la WSN. Con ello se ha generado y establecido un subsistema de vigilancia, alternativo al esquema basado en proveedores de conectividad, de seguimiento y control desde Internet. Interconectar los sensores a Internet genera infinidad de oportunidades en cuanto a las aplicaciones y a los servicios, que pueden ser escenificados en los nuevos modelos emergentes de operación.

En diferentes trabajos sobre la aplicación de las WSN, las tareas de investigación y desarrollo han sido llevadas a cabo en paralelo por diferentes grupos, y muchas veces en forma aislada; con lo cual se ha imposibilitado establecer un marco global unificado sobre el tema; no obteniéndose tampoco aplicaciones prácticas instaladas.

\subsection{Arquitectura del sistema}

La ilustración 6.1 muestra la arquitectura de la red instalada para el presente trabajo; consistiendo en tres niveles básicos: infraestructura, servidor y usuario. 


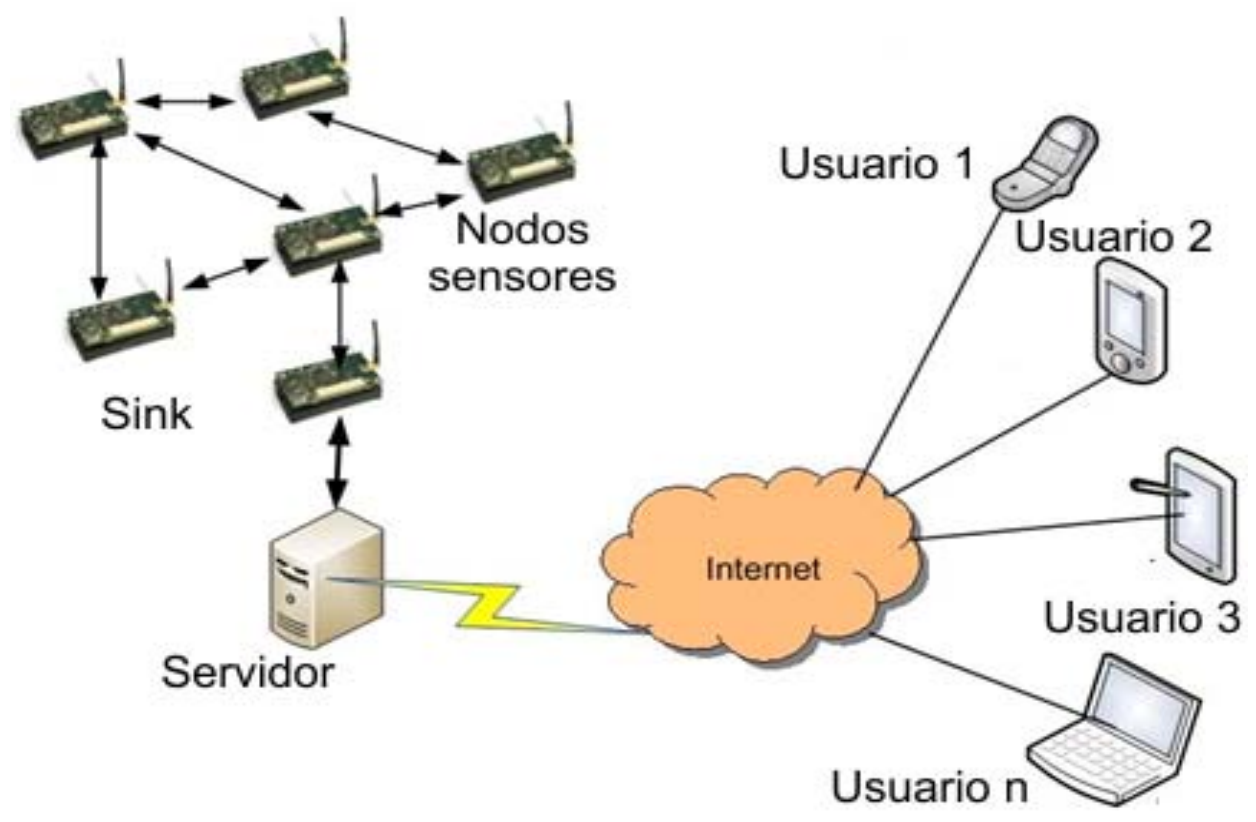

Ilustración 6.1 Arquitectura de la implentación física

En el nivel de infraestructura se encuentran una serie de nodos sensores interconectados. Uno de ellos cumple las tareas de sink. Estos equipos se auto organizan formando una WSN, la que genera datos en forma continua, enviando los paquetes correspondientes al sink. La información capturada por los sensores temperatura, luminosidad y humedad, es transmitida por la WSN. En esta capa de la arquitectura importa el modo de medición, el reporte generado y los protocolos de ruteo implementados.

El servidor instalado captura cada paquete enviado por los nodos de la WSN, utilizando como gateway al nodo sink que se encuentre interconectado al servidor por una interfaz USB. Desde allí reenvía la información a los programas correspondientes para análisis, cálculos y visualización de la información. El servidor difunde la información procesada tanto localmente, como a Internet; desde donde los usuarios 
finales, el tercer nivel de la arquitectura, pueden acceder a la información generada.

En el tercer nivel, los usuarios, pueden acceder a la información por medio de interfaces web, y así recoger y visualizar los paquetes ya procesados en tiempo real. Los usuarios finales son autorizados solamente a consultar los datos, no pudiendo realizar cambios en los parámetros del sistema.

Si bien algunos aspectos de las WSN son genéricos, es importante considerar los aspectos específicos de las aplicaciones a implementar, más aun cuando se tratan de sensores medioambientales.

\subsection{Hardware}

En una WSN los sensores pueden capturar valores y enviar los paquetes de información de alguna de las tres maneras siguientes: de forma temporal al momento de captura, enviando los paquetes de información utilizando alguna manera de sincronización en períodos preestablecidos; por eventos, cuando se realizan las tareas anteriores ante la ocurrencia de un evento predeterminado; ó por requerimiento cuando es solicitado por un usuario final, o por medio de un timer.

En nuestra red de monitoreo experimental no existen inconvenientes en cuanto a la demanda energética de los nodos, debido a la tecnología que se ha aplicado en el ensayo. Se han configurado cada uno de los nodos de manera que se efectúe periódicamente una medición y reporte de parámetros.

Como se ha indicado, a mayor frecuencia de sensado, mayor consumo energético y menor vida útil de la red. A frecuencias menores se presenta generalmente una falta de sensibilidad ante la variación de los valores de parámetros colectados. 


\subsubsection{Plataforma seleccionada}

El observador en una WSN está interesado en el seguimiento de ciertos fenómenos, bajo algunas restricciones de latencia y la precisión. En una típica red de sensores, cada uno de los equipos individuales realiza la medición de valores locales, y difunde esta información hacia los otros miembros de la red y, eventualmente, al observador. Las mediciones realizadas por los diferentes equipos son muestras discretas del fenómeno físico bajo observación, que dependerá tanto de la precisión y exactitud la tares de los sensores; como de la ubicación física de los mismos. En nuestro trabajo no hemos profundizado sobre el tema de ahorro energético, debido a que cada uno de los nodos instalados en la red posee un sistema de captación y almacenamiento de energía solar; por lo que la autonomía de la WSN es indefinida. Además, considerando que la información es remitida a una estación base, la escalabilidad de la red instalada es excelente.

Como plataforma base para el proyecto se han utilizado equipos con un módulo principal iSense [16]. El hardware iSense se proporciona junto a un firmware operativo y de red modular, permitiendo la generación de aplicaciones pequeñas pero completas; y provee una base sólida para el desarrollo rápido de aplicaciones. Brinda una API $\mathrm{C}++$ para el nodo hardware, funcionalidades de sistema operativo y una amplia variedad de protocolos de red.

El sistema de software iSense [15] incluye un número variado de servicios y protocolos listos para usar, tales como ruteo, sincronización de tiempo, programación "en el aire", etc.

Se utilizaron otras computadoras personales como plataformas de desarrollo de las aplicaciones que luego serían transferidas a los dispositivos sensores. En estos equipos se debieron instalar los paquetes 
indispensables para permitir el desarrollo y compilación de las aplicaciones en $\mathrm{C}++$.

En los equipos de desarrollo se utilizó una plataforma PC+Linux en sus distribuciones Ubuntu y Debian. Se instalaron los paquetes make, cmake and gcc++. La plataforma iSense, que incluye al microprocesador Jennic, precisa para el desarrollo de aplicaciones el compilador ba-elf-g++, que asegura la integración perfecta de las librerías.

En la ilustración 6.2 se puede observar el tamaño comparativo del equipo, referido a una mano. El nodo de la figura está compuesto por módulos de medición intensidad luminosa y temperatura. También se observa la interfaz USB para control e instalación de programas compilados. El tamaño final del módulo es determinado predominantemente por el tamaño del porta baterías, o el dispositivo de captación y almacenamiento de energía.

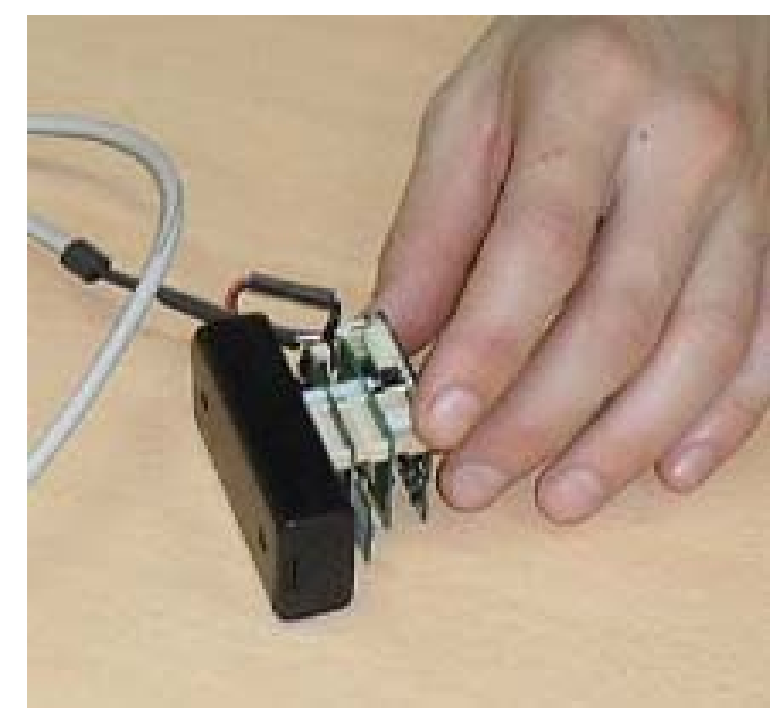

llustración 6.2 Nodo sensor iSense con porta baterías y cable USB 


\subsubsection{Módulos utilizados durante los experimentos}

\subsubsection{Módulo principal}

El dispositivo, presentado en la ilustración 6.3; integra un procesador Jennic JN5139 con un sistema radial de 2,4 GHz compatible con normas IEEE 802.15.4, con ancho de banda de 250kbit/s, sistema de encriptación por hardware AES, ToF ${ }^{1}$ para cálculo de distancias entre nodos, 192kB de ROM, 96kB of RAM, así como una variada posibilidad para la utilización de periféricos analógicos y digitales.

Las características físicas y mecánicas del módulo principal iSense, como de los demás módulos que han sido empleados en este trabajo se encuentran detalladas en el Apéndice F.

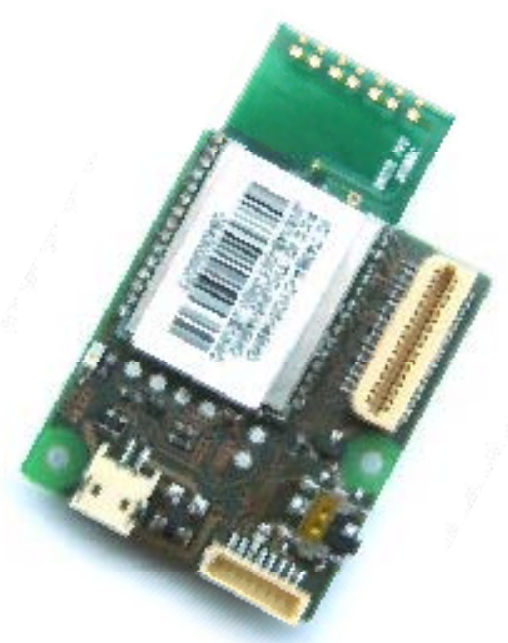

Ilustración 6.3. Módulo principal iSense

\footnotetext{
${ }^{1}$ Time of Flight
} 


\subsubsection{Módulo Gateway}

Proporciona un medio de conectividad a otros sistemas a través de USB y RS-232. Con él se realiza el intercambio de datos y la programación en serie de módulos básicos conectados. El conector USB también puede utilizarse para alimentar de energía a los módulos integrados al nodo.

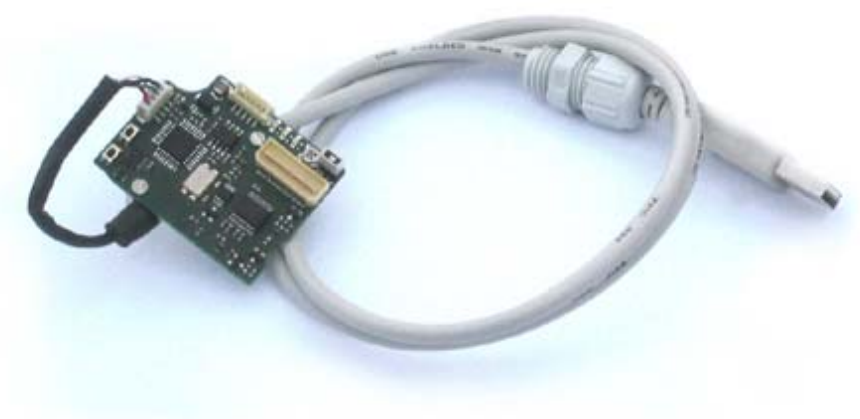

Ilustración 6.4. Módulo Gateway

\subsubsection{Módulos de seguridad}

El sensor de seguridad iSense cuenta con un módulo detector de infrarrojos pasivos (PIR), utilizado para detección de objetos móviles que poseen una temperatura diferente a la ambiental a distancias de hasta 10 metros. El acelerómetro de 3 ejes puede ser configurado de manera de detectar aceleraciones hasta $\pm 2 \mathrm{~g}$ ó $\pm 6 \mathrm{~g}$., siendo posible adicionar un módulo para cámaras de alta resolución. 
En la ilustración 6.4 se muestran los sensores de movimiento y la placa con los acelerómetros, los que juntos conforman el módulo de seguridad de iSense utilizado en este trabajo.

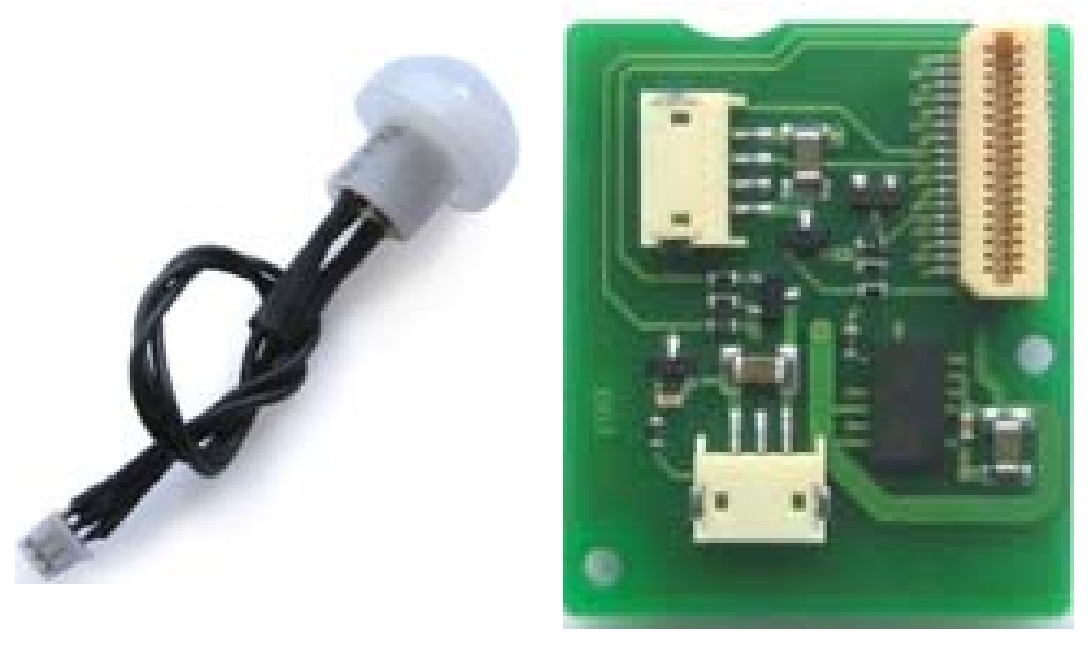

Ilustración 6.5. Módulos de Seguridad utilizados

\subsubsection{Módulo climático}

Este módulo proporciona información de gran precisión cuando se mide temperatura, humedad relativa y presión barométrica (ilustración 6.6).

\subsubsection{Módulo ambiental}

El módulo sensor ambiental (ilustración 6.7) combina un termómetro y un sensor de intensidad luminosa para la vigilancia ambiental. El termómetro proporciona un valor umbral configurable, así como un valor de histéresis.

El sensor de iluminación ofrece dos tipos de mediciones, uno emitido por un sensor sensible a luz en general, y otro por un sensor de luz infrarroja. 
Su diferencia indica la intensidad luminosa teniendo en cuenta la luz visible para humanos solamente. El termómetro es idéntico al del módulo climático, y el sensor de intensidad luminosa no posee límites de captura.
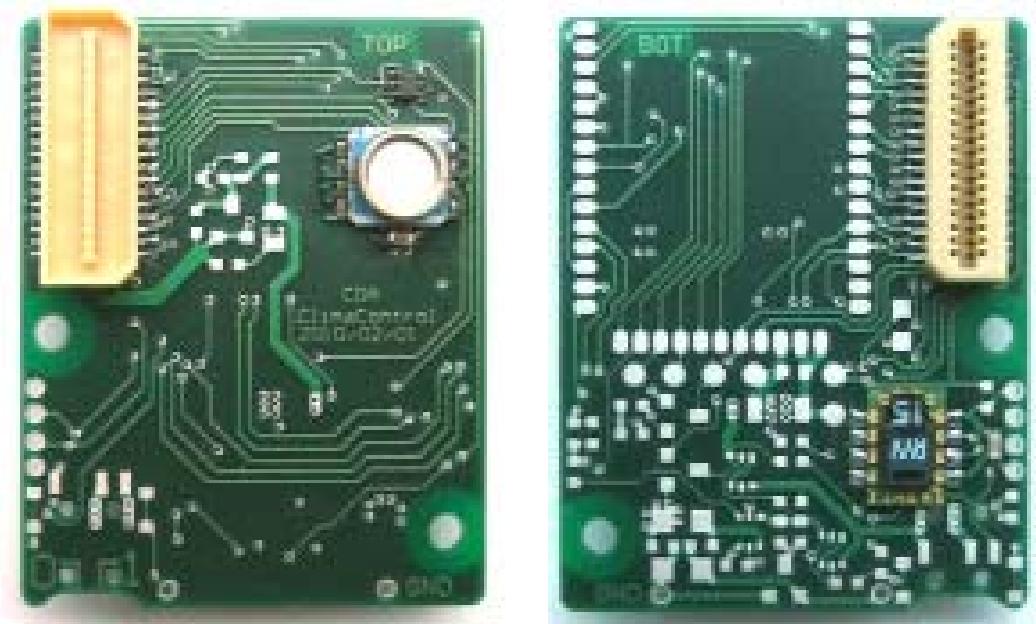

Ilustración 6.6. Módulo climático utilizado

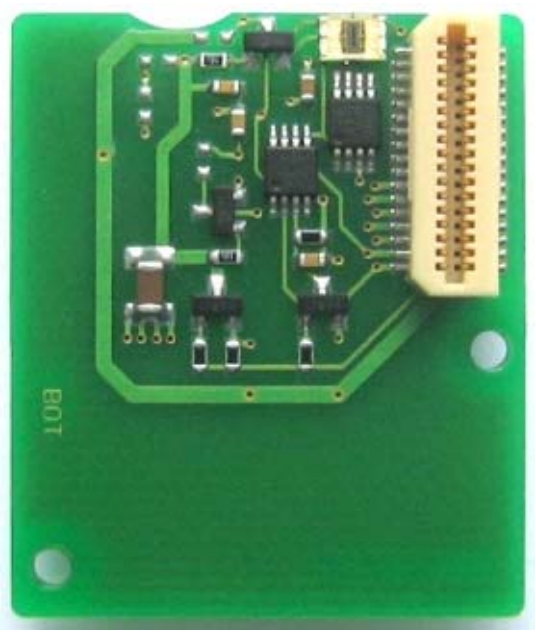

Ilustración 6.7. Módulo ambiental utilizado 


\subsubsection{Sistema de aprovechamiento de energía solar}

Por medio de la colección de energía solar, y almacenándola en una batería recargable, este módulo permite el aprovechamiento de la misma, por lo que la autonomía de los nodos es indefinida. En la ilustración 3.11 hemos visto los componentes del sistema de aprovechamiento de energía solar que se han utilizado en el presente trabajo.

Este sistema consiste en un panel solar, un módulo de administración de energía, una batería de ion-litio recargable, y una caja estanca de protección para todo el equipamiento. La ilustración 6.8 muestra ambas caras del módulo de administración.
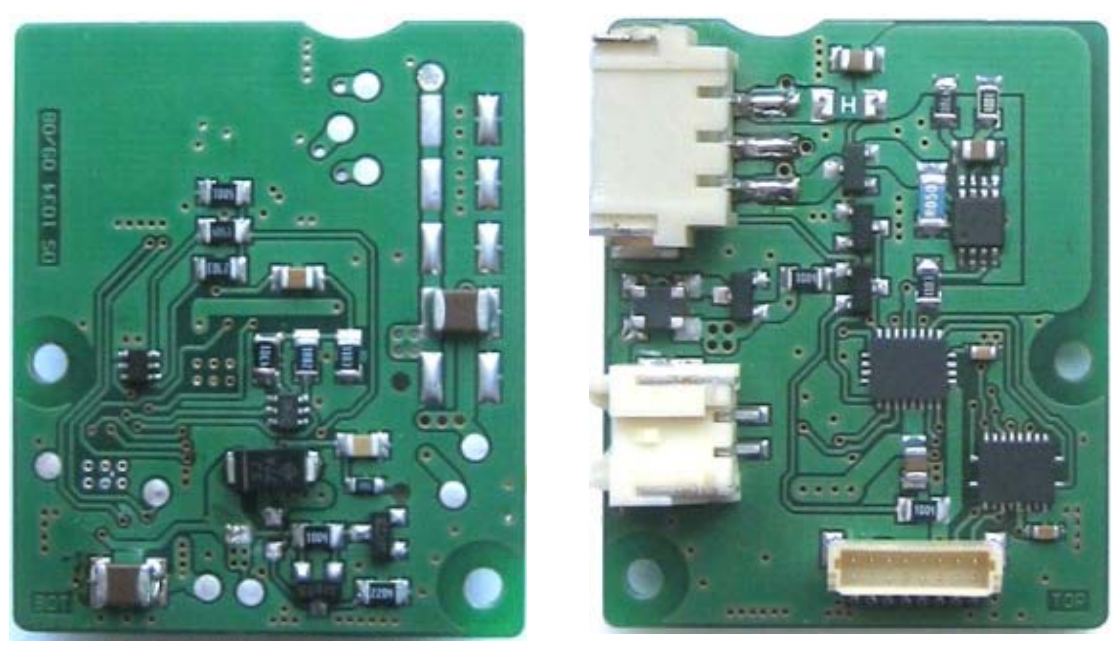

\section{llustración 6.8 Módulo de administración de energía}

El módulo de administración de energía, no solo controla los flujos de energía necesarios para el normal funcionamiento, sino provee un detallado informe de la energía acumulada en la batería, procediendo a la 
acumulación en un contador de los ciclos de carga y descarga, como también información referente al consumo instantáneo y la temperatura. El esquema de funcionamiento del modulo en modos de carga y almacenamiento se ha presentado en la ilustración 3.6. En la ilustración 6.9 se muestra el montaje de uno de los nodos en la caja estanca con la batería y módulos.

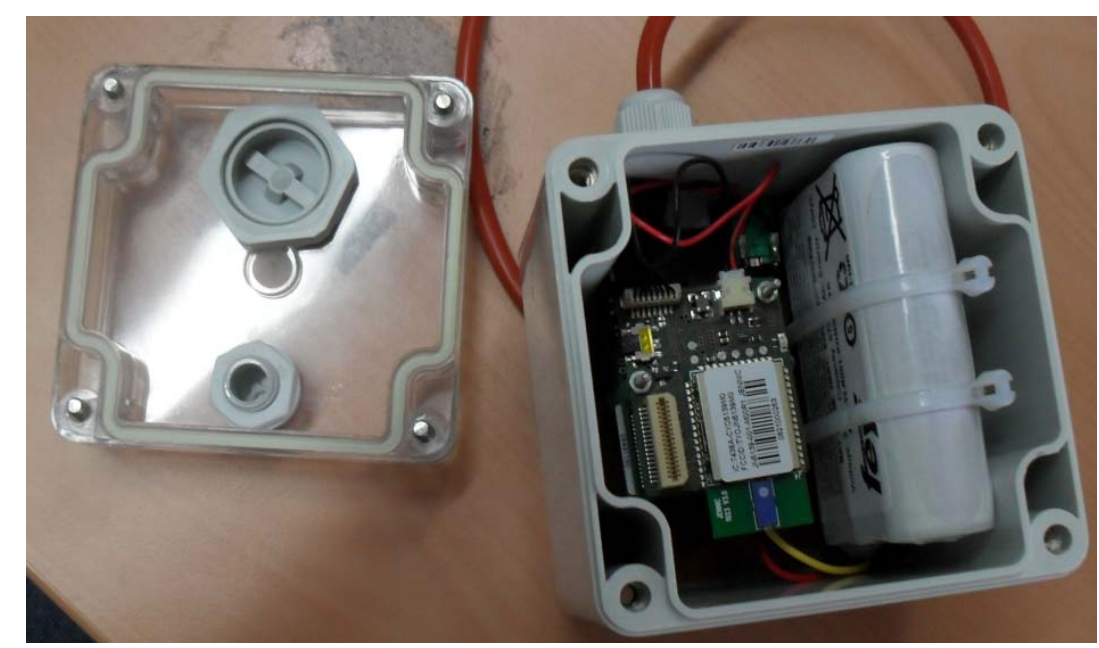

llustración 6.9 Caja estanca con la batería y módulos montados

El módulo se completa con el panel de colección de energía solar, cuyas características se indican en el Apéndice $\mathrm{F}$

\subsection{Software}

El lenguaje de programación que se ha utilizado para el desarrollo de las aplicaciones es $\mathrm{C}++$. El compilador tanto en entorno Linux como Microsoft Windows ha sido GNU Compiler Collection [207]. GCC, incluye front-ends para C, C orientado a Objetos, C++, Fortran, JAVA y ADA; así como las librerías para estos lenguajes (libstdc, libgcj, etc). 
Como entorno de simulación se ha utilizado a Shawn [201]. Para pruebas y configuración de aplicaciones ha sido adoptada la herramienta iShell [208].

La herramienta iShell para análisis, programación y operación de los sensores, es la contraparte del firmware de red y de operación [209] de los nodos iSense. Uno de los beneficios de la utilización de iShell es que permite la simulación de aplicaciones en Shawn sin ningún tipo de esfuerzo extra, ya que las aplicaciones se compilan de acuerdo al destino de la aplicación final, ya sea nodo o simulador.

De manera de poder mantener todas la herramientas en un contexto racional, simplificando así las tareas de programación, depuración y compilado de programas; se ha integrado Shawn e iShell a Eclipse [210]. En la ilustración 6.10 se muestra la consola de Eclipse con Shawn ya importado como así también el entorno de desarrollo de aplicaciones para los sensores iSense.

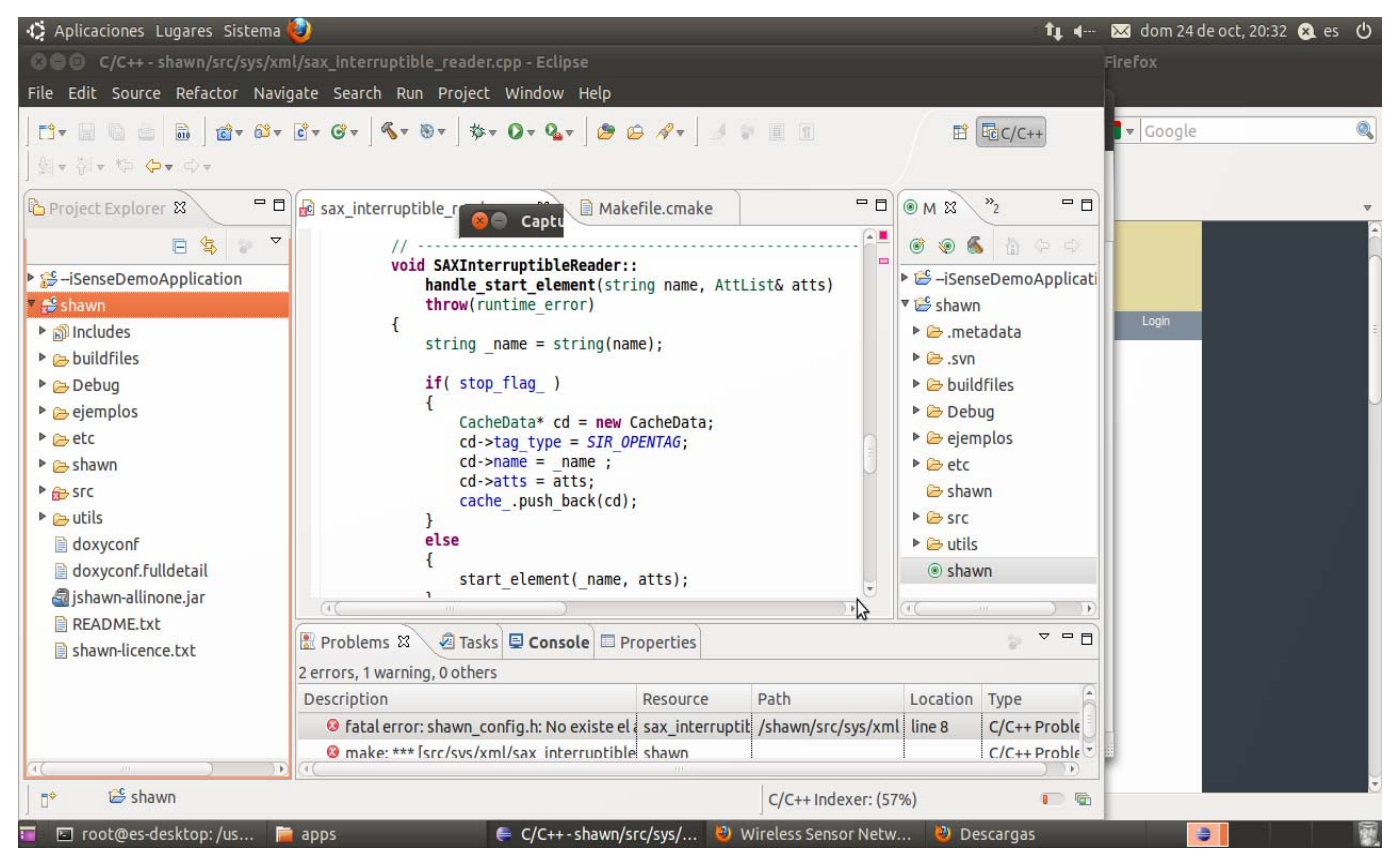

Ilustración 6.10 Entorno de Eclipse con Shawn y entorno de desarrollo de aplicaciones 


\subsubsection{Shawn}

En el capítulo anterior hemos visto que las técnicas más empleadas en el análisis de la performance de las WSN son el modelado analítico, la simulación, la emulación, las experiencias pilotos y la instalación en ambientes reales. Imran et. ál. [211] ha presentado estos puntos esquemáticamente en la ilustración 6.11.

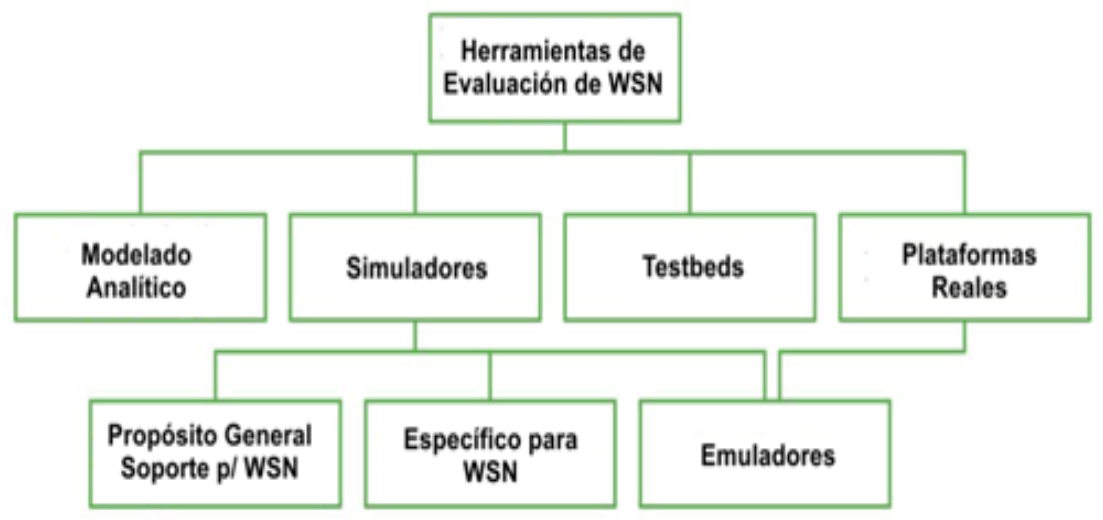

Ilustración 6.11. Herramientas de evaluación de rendimiento de WSN según Imran

Para simular la vida real, las herramientas de simulación realizan presunciones y simplificaciones para el modelaje y predicción, para así poder ser utilizadas en el ámbito de las WSN. Por esta razón algunas de las herramientas son inapropiadas; considerando la complejidad de las WSN, y a la naturaleza innovadora de la tecnología.

Los modelos, cuando son muy simplificados, conducen ineludiblemente a inconsistencias e inexactitudes no deseables. La mayoría de las redes de sensores normalmente están formadas por un gran número de nodos (cientos o miles), por lo que realizar un estudio en campo real es prácticamente imposible. Tampoco se puede realizar un estudio a través de medios analíticos, ya que son métodos complejos, costosos y muy largos. 
Todas las implementaciones de simulación, pretenden ser lo más realísticas posible cuando simulan efectos físicos, datos, codificación de mensajes, efectos de la interferencia inalámbrica, limitaciones de procesador, etc. Sin embargo, una alta eficiencia y exactitud se traduce inequívocamente en largos tiempos de procesamiento.

Se ha seleccionado Shawn como simulador por ser el más completo, ya que es capaz de simular comportamientos e interacciones a varios niveles. Shawn es escalable, soportando varios millares de nodos en una amplia variedad de configuraciones. Es fiel, porque es capaz de representar el comportamiento de la red en intervalos sucesivos representando las interacciones en el nodo, y entre los nodos. Por último se ha seleccionado Shawn por ser transparente, dado que el código utilizado en la simulación, se implementa directamente en un nodo real. La arquitectura interna de Shawn difiere sustancialmente de la que presentan los otros simuladores. Shawn está diseñado para el manejo de redes de sensores a gran escala, donde las prestaciones de los otros paquetes no son buenas.

\subsubsection{Simulaciones efectuadas}

En la primera simulación, hemos utilizado el procesador HelloWorld. Este es el más simple de los procesadores de Shawn. Cada nodo realiza las tareas de arranque y envío de un mensaje de un tipo especial. Los nodos que reciben el mensaje anterior muestran su identificación y la del remitente. Si el nodo no recibe mensajes en cinco rondas de simulación, el nodo imprime la identificación de los nodos vecinos conocidos y luego se desactiva.

Para la inicialización de la simulación Shawn precisa de un archivo de configuración que debe poseer información precisa, como el mostrado en la ilustración 6.12. En la primera línea se genera el mundo donde realizará 
sus tareas el nodo correspondiente. Se indica también el modelo de borde que permite representar gráficamente a la red. Luego se lista el modelo de comunicación que adoptará Shawn, siendo en este caso el de gráfica de disco, con un alcance igual a la unidad. Los nodos pueden comunicarse entre sí, siempre que su ubicación se corresponda con una distancia menor que el valor "range".

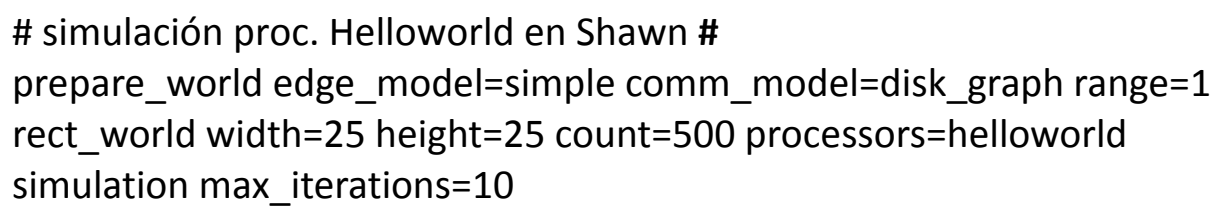

llustración 6.12 Archivo de configuración de Shawn para la simulación con procesador Helloworld

La ilustración 6.13 muestra la consola de inicio de la simulación en donde se observa la comunicación entre los distintos nodos en el entorno de simulación, por ejemplo que el nodo 2 (v2-SWjKZBE) se comunica con los nodos 38, 94, 167 y 350. En el Apéndice $A$ se ha lista el archivo de configuración con el cual se ha corrido la simulación precedente.

Existe una vaga diferencia en la literatura referente a los términos entre localización y posicionamiento. La mayoría de los autores se refiere a posicionamiento cuando el dispositivo recibe una coordenada específica de referencia global, típicamente luego transformada en longitud y latitud. Por otro lado localización es utilizada con objetos relacionados a referencias locales, y por medio de las cuales inferir distancias y ángulos muy precisamente. 


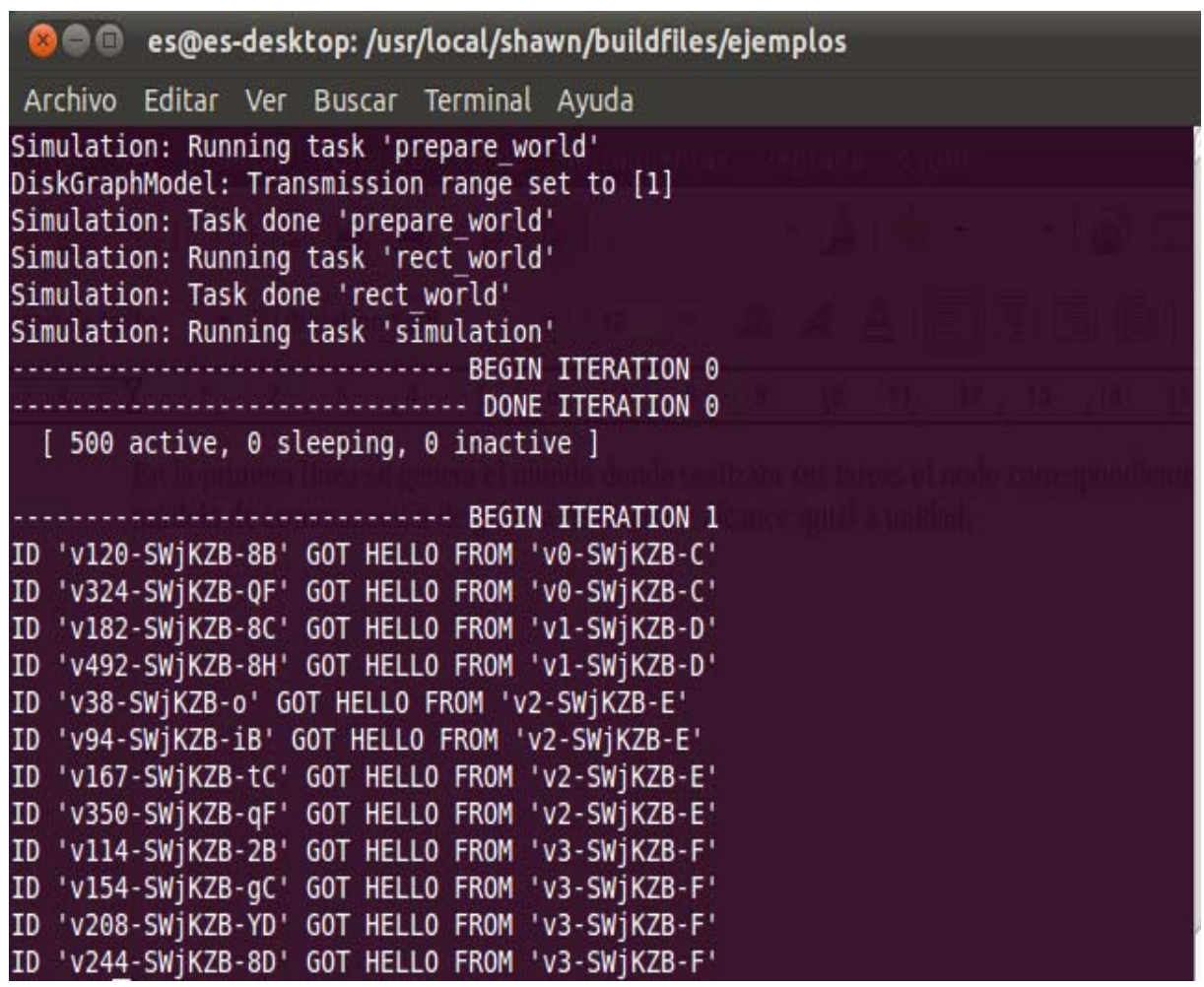

Ilustración 6.13. Pantalla de inicio de simulación

Para una nueva simulación, en este caso de localización, se han distribuidos aleatoriamente 200 nodos en aproximadamente $2 \mathrm{Km}$ cuadrados de superficie. Los nodos simulados son tipo iSense con un alcance radial supuesto $200 \mathrm{~m}$. Doce de los equipos conocen su posicionamiento porque poseen GPS. Los demás deben ubicarse por medio de medición de tiempos de envío de los mensajes. El resultado de la simulación se ha transcripto en el Apéndice B.

En la figura 6.13 se puede observar a los nodos con GPS (nodos ancla) como puntos negros. Estos "conocen" su ubicación geográfica. Los nodos de color gris son aquellos que calculan su posición por algoritmos que los relacionan con la posición de los nodos "ancla". Se observan líneas adicionales desde los puntos grises hacia diferentes ubicaciones en la 
gráfica. Los puntos grises indican la posición real, mientras que la posición unida por el segmento es la posición en la que el sensor estima que está ubicado.

En el Apéndice $B$ se presentan los resultados de la simulación donde se muestra que 191 nodos han podido calcular su posición respecto a los nodos referenciales. Sumados los 9 nodos "ancla", se alcanza el 100\% de los nodos instalados que se encuentran ubicados geográficamente.

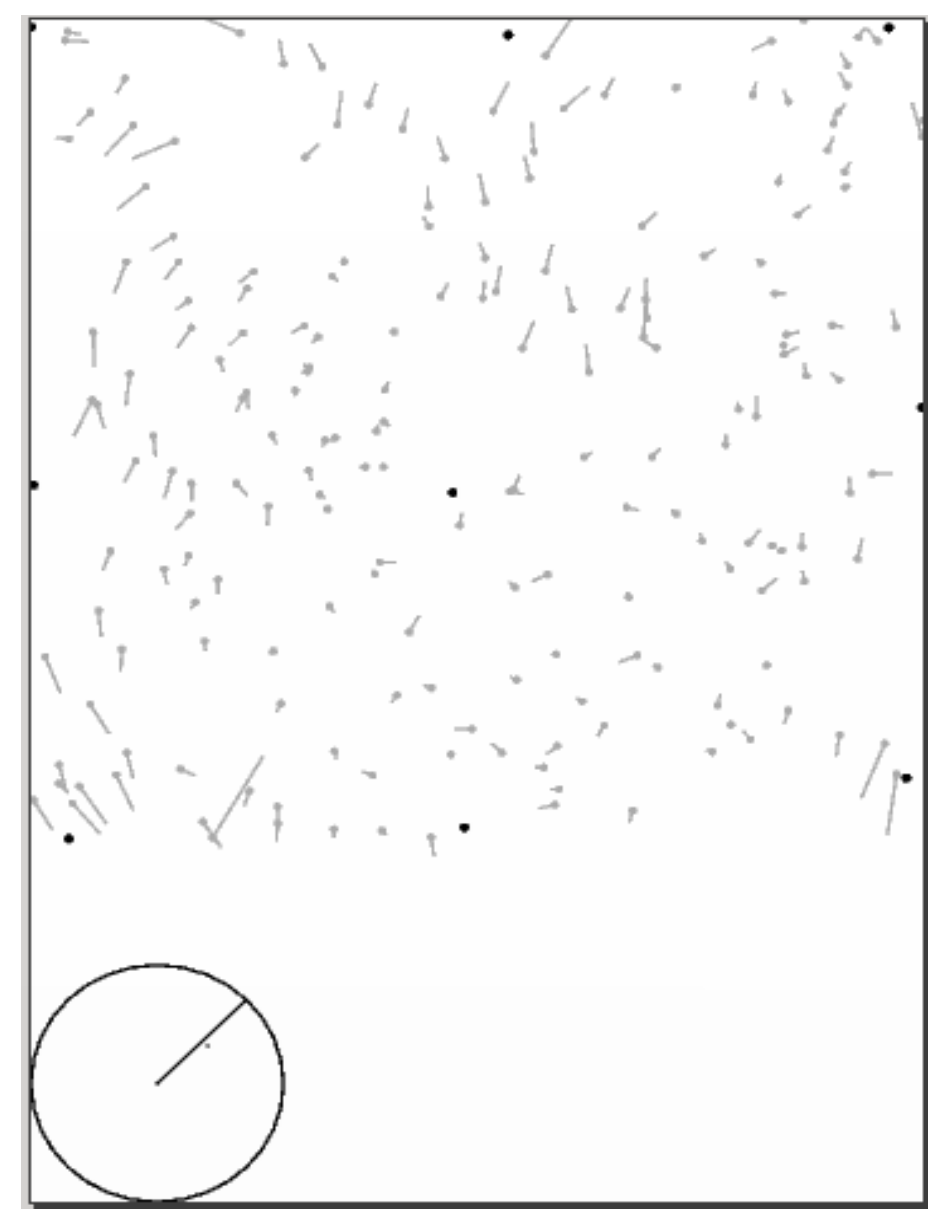

Ilustración 6.14. Simulación de localización en Shawn 
Otros datos importantes que se observan en el Apéndice $B$, tienen que ver con la estadística de la simulación efectuada. Allí se indica entre otros valores que la distancia media absoluta desde la posición real de cada nodo alcanza aproximadamente al $15 \%$ promedio del rango de comunicación, que el número promedio de vecinos por nodo alcanza a 11,22 (desde 3 a 25) y la cantidad de mensajes intercambiados por los nodos son casi cinco mil.

\subsection{Programación y Configuración de los nodos sensores}

Se ha instalado el firmware para nodos iSense, realizando todas las actualizaciones que correspondieran desde el sitio de Coalesenses $\mathrm{GmbH}$ [212]. La selección del firmware no es caprichosa, sino que ha debido seleccionarse como compatibilidad con hardware que se disponía para el ensayo, procesadores Jennic JN5139R1.

El sistema operativo de desarrollo de iSense es $\mathrm{C}++$, por lo que es orientado a objetos y alocación dinámica de memoria. Este sistema operativo provee la funcionalidad de haber implementado un gran número de protocolos tales como protocolos MAC, de ruteo y de sincronización de relojes. Además provee de una herramienta fundamental apara nuestro proyecto, la "programación en el aire". Con esa herramienta se evita la tarea iterativa de realizar la instalación de las aplicaciones nodo por nodo. Así se han desarrollado aplicaciones para establecimiento de WSN donde se han instalado las aplicaciones para control de sensores alimentados a energía solar, sensores infrarrojos pasivos, acelerómetros, temperatura, nivel de iluminación, presión atmosférica y detección de vehículos.

La herramienta iSense ha sido importada a Eclipse, conjuntamente con Shawn. En ese entorno es simple seleccionar el archivo de código en $\mathrm{C}++$ (.cpp) y seleccionando el tipo de procesador a utilizar por los nodos, se genera el archivo ejecutable, compilando y enlazando con las librerías 
correspondientes. En este punto se selecciona si la aplicación se destina a JN5139R1 ó para el simulador Shawn. La ilustración 6.15 muestra la estructura de directorio instalada por default por el firmware de iSense.

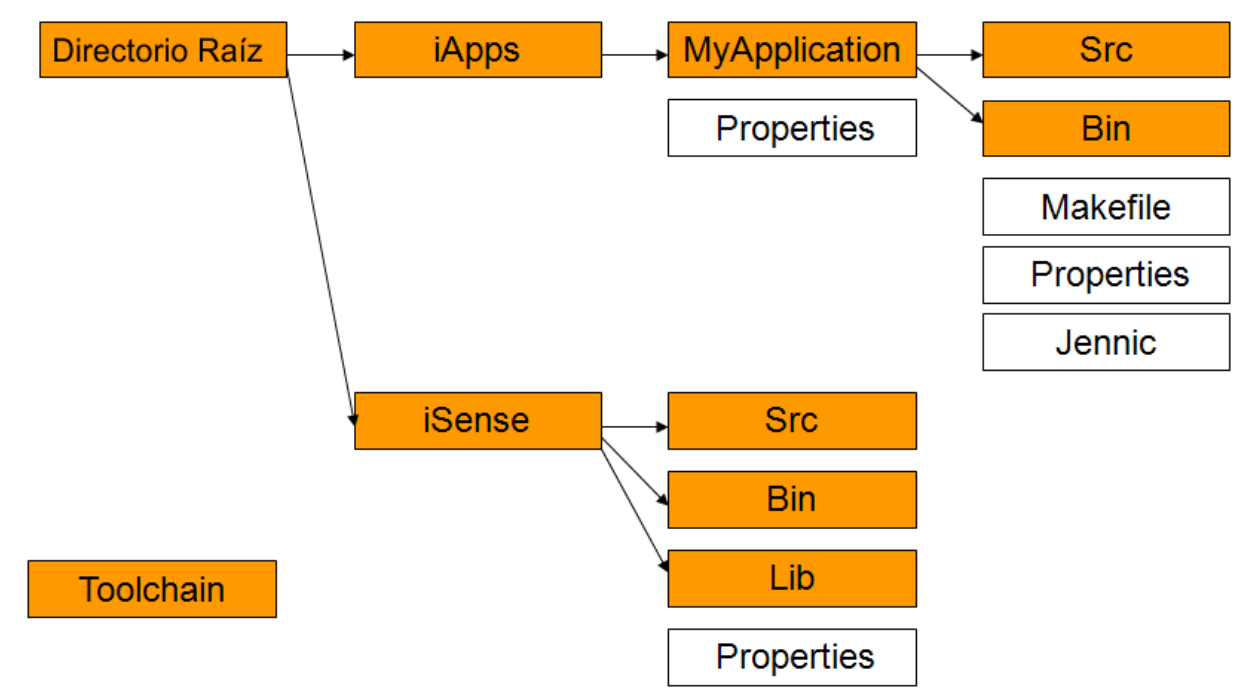

Ilustración 6.15. Estructura de directorio instalada por el Firmware iSense

Cada vez que se compila y enlaza una aplicación, el utilitario indica el tamaño de la aplicación generada de manera de evitar sobrepasar los límites físicos propios del hardware donde va a ser instalado.

En la ilustración 6.16 se muestra una pantalla de Eclipse indicando el éxito de la compilación y enlace a librerías de un programa demo, cuyo listado de código se puede visitar en el Anexo C. En la pantalla inferior de la ilustración se observa que la aplicación ejecutable posee un tamaño aproximado a $70 \mathrm{~KB}$.

La ilustración 6.17 muestra la interface de Eclipse en el momento de generación de un archivo ejecutable, cuya función es capturar los datos por medio de módulos climáticos y ambientales. 


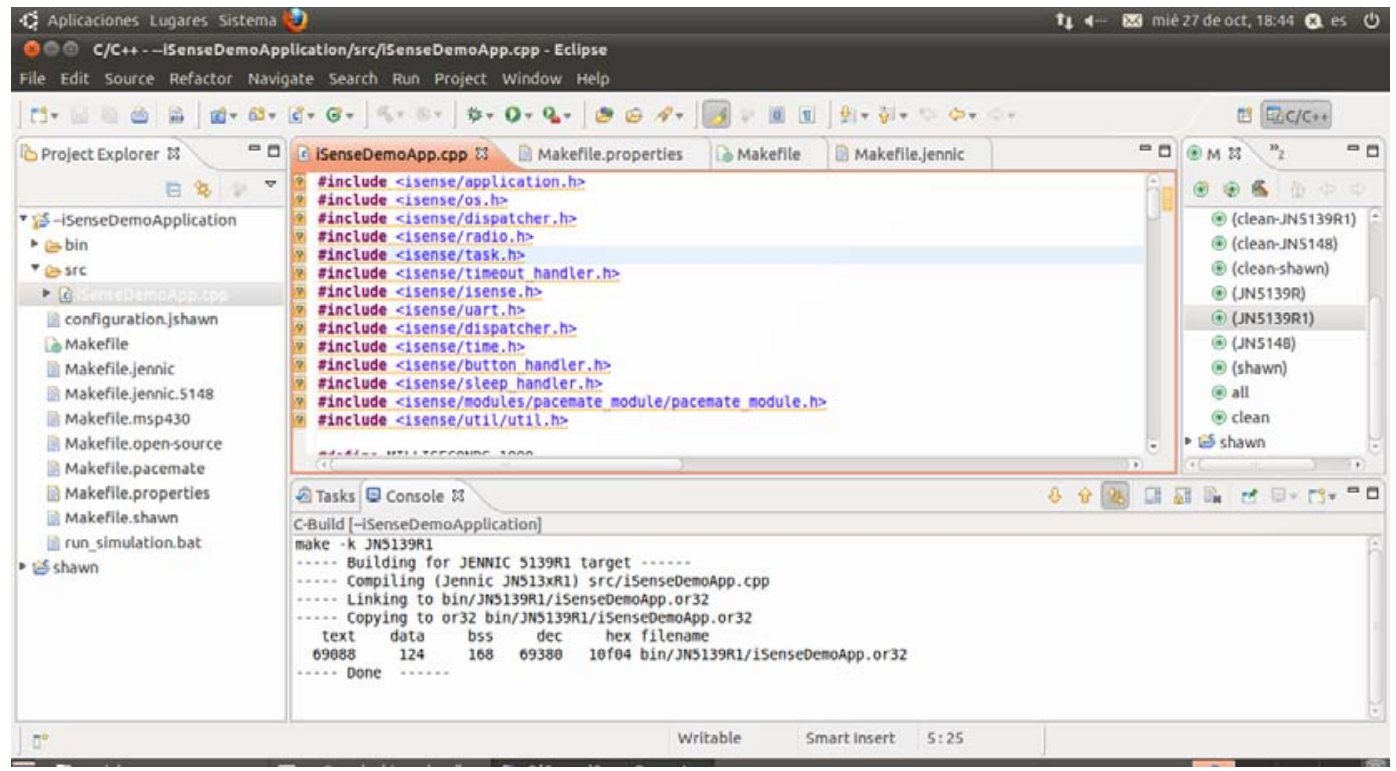

Ilustración 6.16 iSense generando aplicación en entorno Eclipse

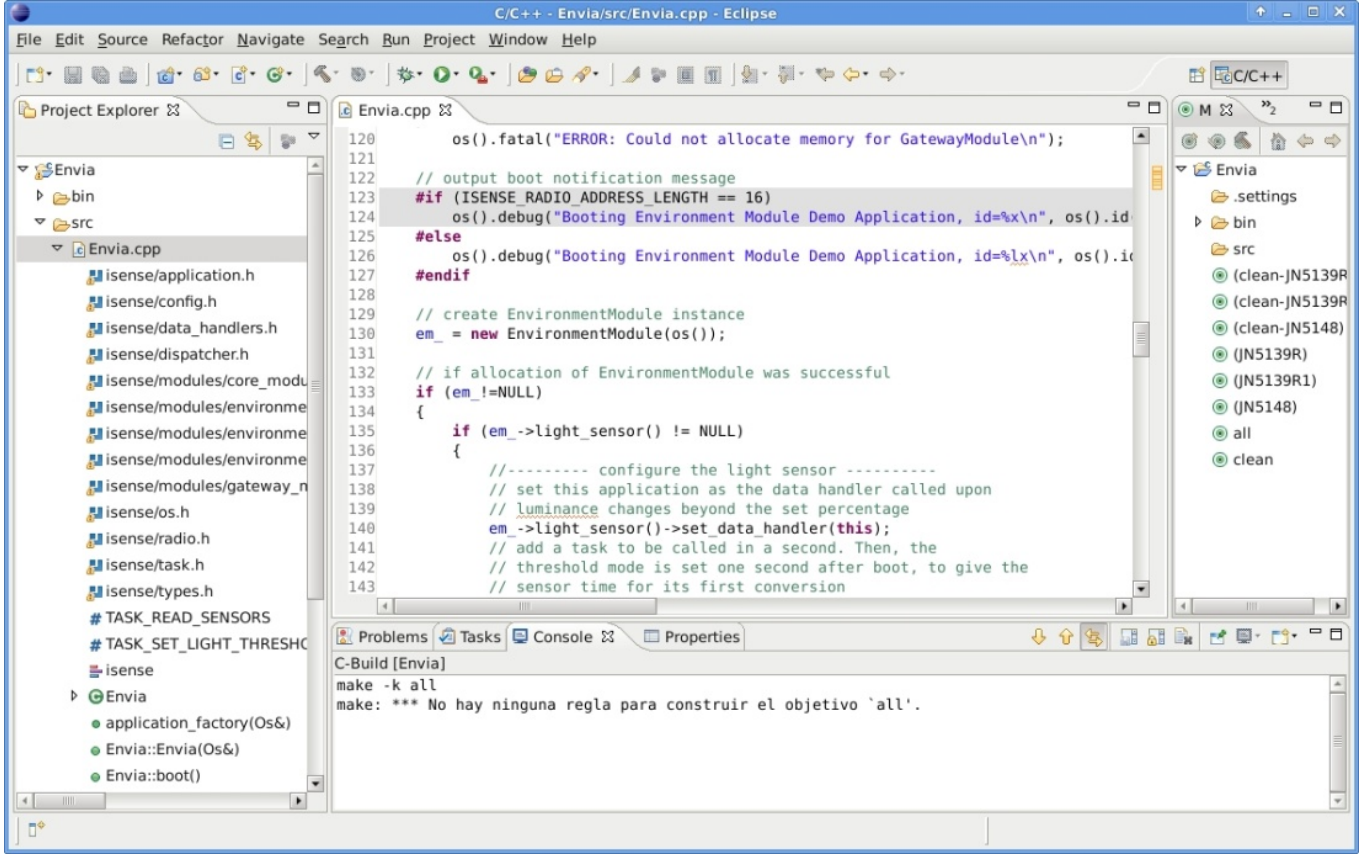

Ilustración 6.17 Creación de ejecutable en entorno Eclipse

Para utilizar los módulos climáticos, que son los últimos que se adicionaron a la red; hubo que establecer una actualización del firmware, 
dado que se reportaron a Coalesenses $\mathrm{GmbH}$ inconsistencias en el comportamiento de iShell con dichos módulos.

El programa envía.cpp se encarga de capturar los datos en los diferentes nodos y son enviados al nodo pasarela y almacenados en una base de datos. El listado del programa envía.cpp se lista en el Apéndice C.

Todas las aplicaciones se han instalado en los nodos sensores por medio del módulo gateway USB instalado sobre una PC de escritorio que corre iShell, o por medio de OTAP (Over the air programation) a través del nodo sink. En la figura 19 se muestra a iShell realizando la instalación del programa ejecutable Envia.bin en el nodo con dirección MAC 1c:22. Referente a las direcciones MAC, los nodos Jennic no poseen direcciones MAC originarias de fábrica, pudiendo ser asignadas de acuerdo a la voluntad del desarrollador. En el caso que el nodo no posea dirección MAC, el nodo indica la dirección ff:ff:ff:ff:ff:ff:ff:ff.

\subsection{Red soporte alternativa para la WSN}

La utilización de las redes sociales ha alcanzado un desarrollo que no se discute a esta altura de las circunstancias. Una red social está constituida por agrupaciones de individuos, la que se establecen de acuerdo a intereses comunes; las que pueden establecerse presencial ó virtualmente. En el último de los casos nos referimos genéricamente a éstos como sitios web sociales.

El logo del sitio de Twitter indica "La mejor manera de descubrir lo que está pasando en tu mundo" [213]. Lo anterior ha disparado la inquietud de verificar si la infraestructura ubicua de esta red social es capaz de soportar una aplicación de redes de sensores. 


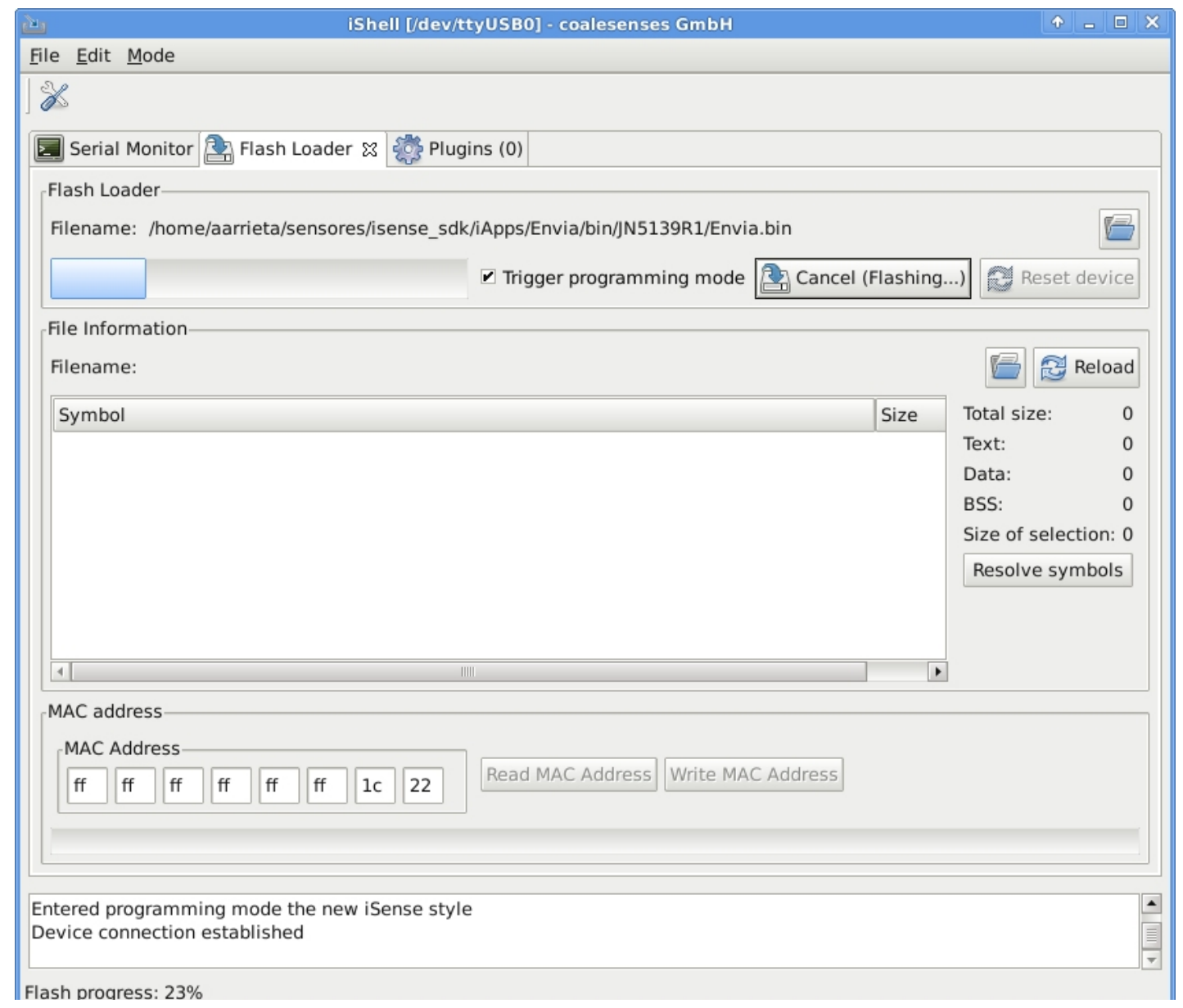

Ilustración 6.18. Ishell instalado aplicación en nodo 1c:22

En la República Argentina se vive una realidad técnica y práctica en lo que respecta a probabilidades de obtener una cobertura eficiente y eficaz de servicio de conectividad, fundamentalmente a cobertura de última milla, del cual depende el consecuente acceso a la red digital soporte. En las WSN, estas redes públicas son el respaldo de infraestructura, debido lo oneroso de utilizar vínculos satelitales.

La más de las veces el servicio de acceso a la red digital soporte es provista por empresas monopólicas, con las cuales es tarea ímproba establecer cierto tipo de Contrato de Nivel de Servicios (SLA). Los 
servicios de calidad son bastante dificultosos de establecer si el proyecto en cuestión se encuentra fuera del de la zona geográfica poligonal determinada por las ciudades de Mendoza, Córdoba, Santa Fe y Buenos Aires y Bahía Blanca.

Como resultado de ello, los proyectos fracasan antes de implementarse, ó son condenados a abonar costos excesivamente elevados por una solución particular. La red de telefonía celular se encuentra mucho más avanzada en ese sentido, lo que permitiría cubrir el déficit indicado.

La pretensión es utilizar características propias de las WSN, y el hecho de la instantaneidad de la red social elegida. Twitter es una plataforma de envíos de mensajes no dependientes del equipamiento [214], manera elegante de decir que es capaz de enviar y recibir mensajes por una variedad diferente de equipos, de manera instantánea.

Utilizar a Twitter como una herramienta de notificación de eventos identificados como importantes, no es un dato novedoso. En el Japón se ha elaborado un algoritmo de procesamiento de los mensajes [215], con el cual se pueden anunciar con suficiente antelación, y una alta probabilidad de ocurrencia, la inminencia de terremotos a lo largo de todo el país; aún antes que el evento sea notificado a la población por la Agencia Meteorológica Japonesa.

Como equipo pasarela a la red Twitter se ha utilizado una computadora personal de escritorio con sistema operativo Debian GNU/Linux con conexión directa a Internet.

\subsubsection{Servicio Twitter}

Para el acceso y autenticación a una cuenta, Twitter utiliza el protocolo abierto de autenticación OAuth [216] aplicado sobre protocolo http. OAuth proporciona un procedimiento para acceder a los recursos de un servicio 
en nombre de un propietario del recurso (tal como un cliente diferente o un usuario final). Twitter ofrece la posibilidad de obtener una ficha de acceso único, mediante uso de la cual se elimina la necesidad de implementar todas las características de OAuth.

Se ha desarrollado una rutina de captura de eventos y generado un texto específico de salida de manera de poder filtrarlo y/o catalogarlo en etapas posteriores. Se ha programado a los sensores para que indiquen por medio de encendido de LEDs específicos cuando se encuentran en modo medición.

Ha sido necesario programar el firmware de dichos equipos para que cumplan con las necesidades de la implementación. Al programarlos se los ha configurado para que realicen una lectura de datos cada diez minutos, evitando así un excesivo tráfico de datos entre el Gateway y Twitter. En caso de variaciones bruscas en las variables medidas sobrepasando limites preestablecidos (tresholds), la programación indica que deben emitir un alerta extra del evento.

La lectura efectuada por cada uno de los sensores se difunde a través de la WSN, utilizando la interfaz de radio de cada nodo. Tanto al leer como al recibir datos, cada nodo imprime el evento por medio de su salida serial.

Con el objeto de simplificar la tarea de programación, y hacer más sencillo el reemplazo de nodos o el agregado de nuevos nodos a la red, se ha programado que todos los nodos realicen la misma tarea de lectura y envío de datos. Esto implica cierta redundancia en la operación, pero asegura la robustez del sistema, confiando en la flexibilidad de este tipo de redes y a su adaptación a cambios en la topología, sin alterar su implementación.

Para publicar en Twitter, se ha utilizado uno de los nodos de la red pasarela, conectado a través del puerto USB a la computadora que actúa 
de servidor. Se ha utilizado un script hecho en el lenguaje Python [217], que se utiliza para conexión de dispositivos seriales, como también realizar operaciones sobre los mismos. Sobre dicho script se han realizado algunas modificaciones para incorporar la funcionalidad de publicar los datos en la cuenta de Twitter (ver Apéndice D).

\subsubsection{Twitter y nuestra WSN}

Twitter ha demostrado ser capaz de cumplir funciones de soporte alternativo de nuestra WSN. Esta podría ser utilizada en casos de necesidad en sitios de despliegue de las WSN que no se disponga acceso a la RDS.

En la ilustración 6.19 se muestran reportes publicados en el sitio de Twitter desde diferentes nodos de la red de sensores desplegada en el Campus Universitario de la Universidad Nacional de Misiones. Los reportes son accedidos por medio de un navegador, pudiendo observarse las distintas direcciones MAC de los nodos generadores de las distintas capturas realizadas. Los sensores en la ilustración reportan la temperatura ambiental en ${ }^{\circ} \mathrm{C}$ y la intensidad luminosa en Lux. La distancia entre cada uno de los nodos en el sitio de despliegue de la red ha variado entre los 10 y 80 metros.

A los efectos ilustrativos de la utilidad de la experiencia desarrollada en la Ilustración 6.20 se muestran los datos on-line recibidos por un celular posicionado a $10 \mathrm{Km}$ del lugar de emplazamiento de la WSN. 


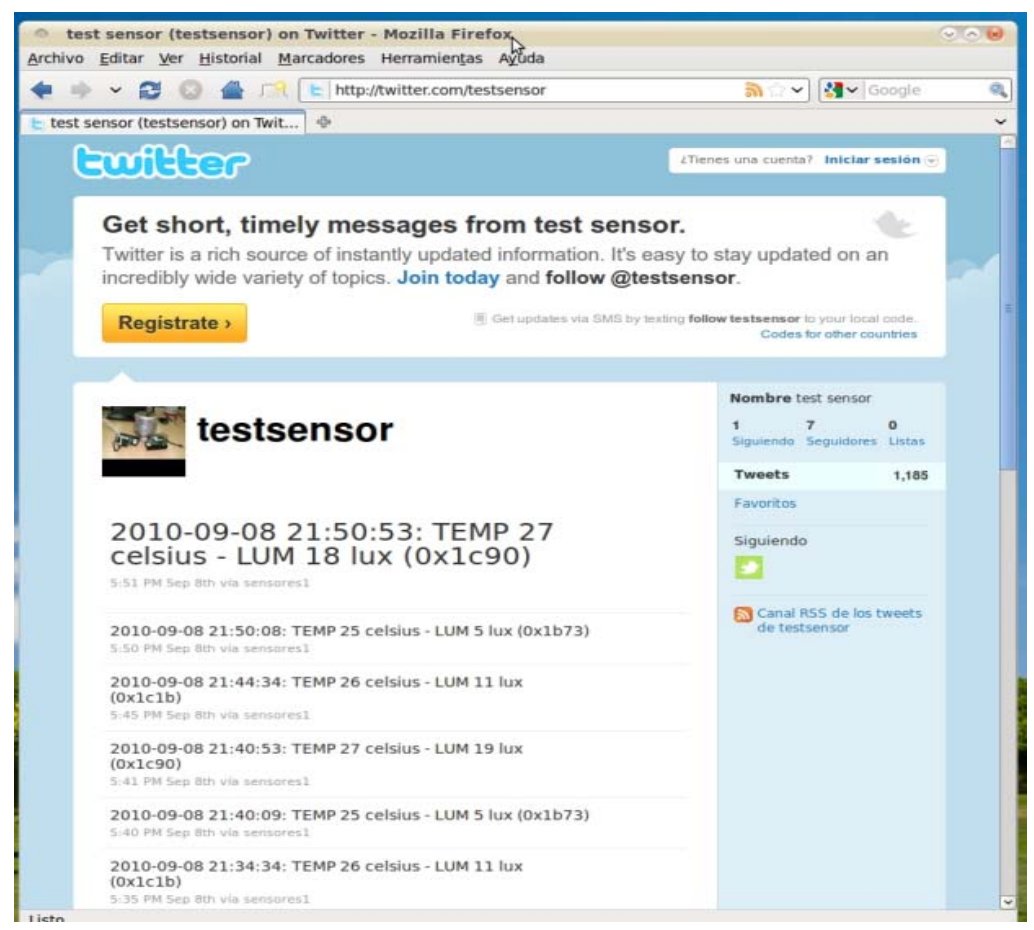

Ilustración 6.19. Reportes de la WSN en el sitio web de Twitter

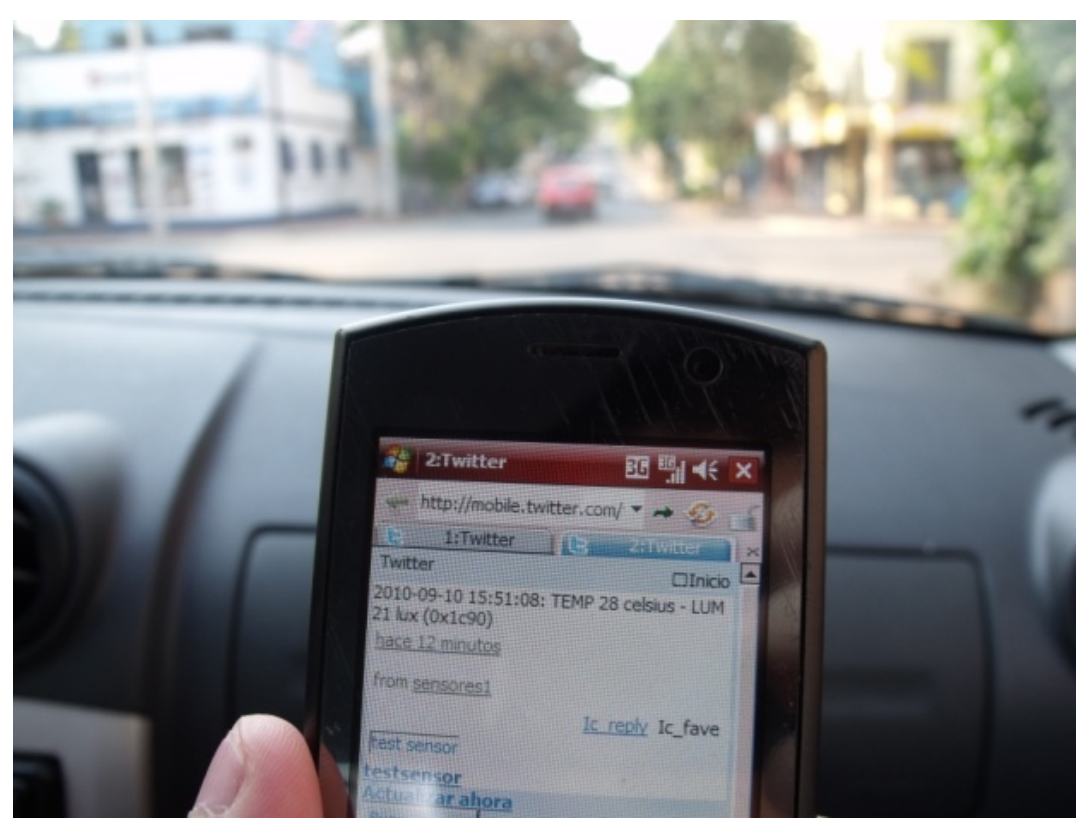

Ilustración 6.20. Datos de la WSN accedidos del sitio de Twitter por medio de teléfono celular 


\subsection{Resumen}

Se ha presentado en éste capítulo todo el hardware y software que se ha utilizado, adaptado, compilado y/o modificado con el fin de alcanzar las metas propuestas para el presente trabajo.

Se han especificado los diferentes módulos, caracterizándolos física y funcionalmente, cado uno de los cuales ha sido implementado en las experiencias prácticas, realizadas a escalas de laboratorio como a escala piloto.

Se ha presentado, de manera concisa y clara, la integración realizada de las diferentes herramientas que fueran seleccionadas para el desarrollo de las actividades del proyecto.

Se explicitan los escenarios utilizados para la simulación y la configuración utilizada para las tareas, detallando los resultados presentados por la herramienta. Se presenta una simulación de una red de doscientos sensores en una superficie de aproximadamente 2 kilómetros cuadrados, donde solamente una fracción de ellos poseen idea de localización por medio de un módulo GPS, en un ejemplo realístico de las tareas y funciones de una WSN.

Se presenta la forma y el método de programación e instalación de la aplicación compilada en los nodos de la red desplegada.

Por último se realiza la presentación y validación de una alternativa de utilización de las redes sociales como soporte a la red de sensores inalámbricos. Este caso de éxito se estableció utilizando la red social Twitter. 


\section{Capítulo 7 : Conclusiones}


as WSN han sido materia de intensivos estudios en los últimos años, donde la mayoría de los resultados obtenidos y publicados son de naturaleza teórica, fuera del contexto práctico que encierra el presente trabajo. Estos resultados solapan varios de los inconvenientes existentes en las implementaciones prácticas de la vida real.

En el presente trabajo de aplicación de WSN para vigilancia ambiental, son imprescindibles mediciones de calidad y enlaces confiables. Uno de los elementos no considerados en los estudios anteriores es considerar a la naturaleza jugando un papel preponderante, debido a la imprevisibilidad de su comportamiento.

Se ha podido evaluar el comportamiento de una WSN en condiciones ambientales reales, habiendo demostrado ser lo suficientemente robusta y adecuada para poder alcanzar los objetivos planteados. A lo largo de todo el proyecto se han encontrado diversas situaciones, inconvenientes y desafíos a los que se han establecido soluciones prácticas. La experiencia de este trabajo nos ha indicado que la administración remota de las WSN es un punto álgido en el desarrollo e implementación de estas redes. Esta línea de trabajo se debería profundizar en investigaciones futuras.

Las WSN, a diferencia de las redes que hayamos estudiado hasta el momento, poseen limitaciones referentes a la vida útil de las baterías y la capacidad de cálculo, dificultando su implementación física. Lo primero está directamente relacionado con la necesidad inherente de energía para su funcionamiento, y a la dificultad que se presenta en el reemplazo de las baterías una vez establecida la red. La potencia de cálculo debe relacionarse con la portabilidad, y escalabilidad de los nodos.

Pero a pesar de ello, las WSN han evolucionado para convertirse en un puente entre el mundo físico de los sensores y actuadores; y el mundo virtual de la información y los servicios. Las WSN conforman una de las 
más activas áreas de investigación, y se estima que se encuentra en la parte ascendente de la onda del éxito comercial. Sin embargo el desarrollo de aplicaciones para WSN es aun complejo, dado que conviven el desafío natural de las aplicaciones distribuidas, y el de la programación integrada. Algunos parámetros extra que complican aun más el escenario son la influencia impredecible del medio ambiente, y el tamaño de este tipo de redes.

Las especificaciones de cada proyecto están íntimamente relacionadas a las modificaciones que deban realizarse a lo largo del mismo, y a que las aplicaciones ideadas para las distintas redes evolucionan a lo largo del tiempo de acuerdo a necesidades puntuales. Normalmente, los cambios en los proyectos se corresponden con implementaciones más largas, más caras e inexactas. Este punto es importante, dado que los veloces cambios experimentados en las WSN son inherentes al proceso de desarrollo alcanzado por la tecnología, y los avances logrado día a día en el tema.

Todo lo anterior debe considerarse en un marco que los contenga, abstrayendo de estas situaciones a los desarrolladores. Por lo contrario no se observa en los desarrollos actuales esta tendencia, sino que podríamos afirmar que cada proyecto se inicializa con el desarrollo de aplicaciones desde un nuevo punto inicial.

Se ha realizado en este trabajo un exhaustivo estudio del estado del arte de las redes inalámbricas y de su evolución histórica, para luego hacer especial hincapié en WSN, analizando los matices, características e innovaciones alcanzadas con la evolución de la tecnología involucrada.

Las herramientas utilizadas, se han integrado en una plataforma, donde Shawn, Eclipse e iShell conviven amigablemente. Además se ha investigado en líneas buscando soportes alternativos para nuestra WSN, para así lograr un soporte de conectividad sustentable, a costos 
accesibles. Se ha experimentado con la especificación de protocolos de alto nivel (ZigBee) para su utilización en radiodifusión digital de bajo consumo, la que encuentra su fundamentación en el estándar IEEE 802.15.4. Con ello, se ha logrado establecer una red piloto que sirve de punto inicial en una línea de estudio de las WSN en la región. La red se encuentra operativa, y es por tanto un logro real del proyecto.

De los resultados obtenidos, tanto en simulación como en la implementación de las redes piloto, se han realizado comparaciones de rendimiento, capacidad y propiedades de cada nodo. Se ha establecido una relación entre el ambiente de las simulaciones, y la realidad; dentro de los límites impuestos por la disposición física de equipos para las experiencias.

Los nodos Jennic han demostrado ser sumamente confiables, y con un de alto rendimiento en los trabajos realizaos en campo y laboratorio.

Los resultados obtenidos son útiles para identificar y caracterizar el rol que juega cada uno de los componentes de hardware, y las aplicaciones involucradas; en las WSN. Se ha utilizado un simulador, con el que se han implementado entornos de cálculo y visuales; logrando de esta manera presentar resultados y estadísticas más agradables y amigables al entorno humano. Conjuntamente se ha integrado Vis a Shawn, dado que la simulación textual pura, no satisface la demanda de ciertos usuarios. Con la utilización de Vis, Shawn clarifica las topologías y las actividades de una simulación de manera de presentarlas en una manera más comprensible y amigable.

El trabajo realizado permite la utilización de tecnologías existentes, tales como las redes sociales, para extender el uso de la aplicación de WSN en nuestro país a lugares sin acceso a Internet, o hacia lugares con accesos sumamente restringidos, utilizando para ello la red de celulares y publicando resultados por medio de SMS. Esto es fundamental al 
momento de proyectar instalaciones en muchas regiones de nuestro país y la región.

La integración con Twitter no solo permite colectar racionalmente la información de los fenómenos, sino que se convierte en una forma de publicar, sociabilizar y transparentar información, que de otra manera es de difícil acceso. Uno de los resultados importantes, para las WSN; tiene que ver con la generación de alertas tempranas en distintas situaciones, de acuerdo a necesidades diferentes. La tecnología utilizada, ha demostrado un grado de integración y escalamiento que ameritan ser tenidos en cuenta para proyectos de contralor de una variedad de eventos, ya que consiste en una tecnología que no requiere una infraestructura de comunicaciones propia, utiliza una API para publicar sumamente cómoda, y con un mecanismo de acceder a los datos publicados por demás simple (por navegador o celular).

\subsection{Trabajo Futuro}

La investigación que se ha realizado durante esta tesis ha alcanzado los objetivos propuestos en la sección 1.3. Sin embargo, el área de investigación tanto de los algoritmos de consumo de energía y los de gestión de información; se han destacado como un ámbito interesante y diverso de las WSN, existiendo muchas líneas de trabajo con las cuales se obtendrían resultados muy importantes. Se vislumbra un amplio campo de trabajo para grupos interdisciplinarios interesados tanto en las redes de datos, como en el procesamiento distribuido.

El trabajo futuro debería concentrarse en una extensión de los entornos de desarrollo existentes, para así mejorar cada uno de los componentes actuales. En Shawn, es primordial incorporar modelos adicionales de implementaciones. Una de las formas de lograr esto, es alentar la 
participación de la comunidad Open Source, con lo que se contribuirá en su crecimiento.

Como trabajos futuros sobre el tema, deberían ser considerados:

- Implementación de seguridad en WSN.

- IPv6 en WSN.

- Simulación de consumo energético.

- Convergencia hacia la colaboración y participación de grupos de tareas con implementaciones actualmente en marcha. 


\section{Bibliografía}

[1]. The computer for the 21st century. Weiser, Mark. s.I. : Scientific American (International Edition), Septiembre de 1991, Vol. 265, págs. 66-75.

[2]. Pervasive computing: Vision and challenges. Mahadev, Satyanarayanan. Agosto de 2001, IEEE Personal Communications, Vol. 8.

[3]. Overview of sensor networks. D. Culler, D. Estrin, and M. Srivastava. August 2004, IEEE Computer, págs. 37(8):41-49.

[4]. Ward, M., Kranenburg, R. y Backhouse, G. RFID: Frequency, standards, adoption and innovation. Department of Design, Goldsmiths College, University of London. London : JISC Technology and Standards Watch, 2006.

[5]. A Survey of Applications of Wireless Sensors and Wireless Sensor Networks. Arampatzis, Th., Lygeros, J. y Manesis, S. s.l. : Intelligent Control, 2005. Proceedings of the 2005 IEEE International Symposium on, Mediterrean Conference on Control and Automation. . págs. 719-724.

[6]. Mica: A Wireless Platform for Deeply Embedded Networks. Hill, J. y Culler, D. Berkeley, CA : s.n., 2002, IEEE Micro, págs. 12-24.

[7]. Crossbow. Crossbow MICA2 868, $916 \mathrm{MHz}$. [En línea] [Citado el: 2010 de Mayo de 15.] http://www.xbow.com/Products/productdetails.aspx?sid=174.

[8]. - . Crossbow MICAz 2.4GHz. [En línea] [Citado el: 2010 de Mayo de 15.] http://www.xbow.com/Products/productdetails.aspx?sid=164.

[9]. - Crossbow TelosB. [En línea] [Citado el: 15 de mayo de 2010.] http://www.xbow.com/Products/productdetails.aspx?sid=252.

[10]. Polastre, J., Szewczyk, R. y Culler, D. Telos: Enabling Ultra-Low Power Wireless Research. [En línea] Computer Science Department - University of California, Berkeley, 2005. [Citado el: 16 de Agosto de 2010.] http://www.polastre.com/papers/spots05-telos.pdf.

[11]. Vázquez, I. Experiencias en la Aplicación de Wireless Sensors Networks. [En línea] [Citado el: 15 de Mayo de 2010.] http://paginaspersonales.deusto.es/ivazquez/ponencias/ivazquez_Aplicaciones WSN_20071115.pdf. 
[12]. Zürich, ETH. BTnodes - A Distributed Environment for Prototyping Ad Hoc Networks. [En línea] [Citado el: 15 de Mayo de 2010.] http://www.btnode.ethz.ch/.

[13]. Beutel, Jan. The BTnode Story. Reflections on Almost a Decade of Mote Class Devices. . [En línea] 2009 . [Citado el: 12 de Julio de 2010.] http://www.mics.org/ZH08/slides/s22a.pdf.

[14]. Freie Universität Berlin, AG. ScatterWeb Homepage. [En línea] [Citado el: 15 de Julio de 2010.] http://cst.mi.fu-berlin.de/projects/ScatterWeb/.

[15]. ScatterWeb. ScatterWeb Hardware und Software zur Bildung einer perfekten Lösung. [En línea] [Citado el: 2010 de mayo de 15.] http://www.scatterweb.com/content/products/index.de.htm.

[16]. Bridging the gap between virtuality and reality . [En línea] 2010. [Citado el: 02 de Septiembre de 2010.] http://www.coalesenses.com/index.php ?page=isense-solar-system.

[17]. Li, Xianjang. Wireless Ad Hoc and Sensor Networks. Cambridge : Cambridge University Press, 2008. ISBN 78-0-521-86523-4.

[18]. PicoRadio supports ad hoc ultra-low power wireless networking. Rabaey, J., y otros. Julio de 2000, Computer, Vol. 33, págs. 42-48.

[19]. D., Culler. Wireless Sensor Networks - the next IT Revolution. Korea : s.n., 2004. 35th Korea Electronics Show.

[20]. WISEBED. Wireless Sensor Network Testbeds. [En línea] 2008. [Citado el: 12 de 05 de 2009.] http://www.wisebed.eu/.

[21]. Meneses González, Salvador. Comunicaciones Móviles: Más allá de la 3G... la 4G. México, D. F. : s.n., 2007.

[22]. Provision of User-Centric Mobile Services in Next Generation Mobile Communication Environment. Bae, JungSook, y otros. 2007. Vehicular Technology Conference, 2007. VTC-2007 Fall. IEEE 66th. págs. 2005-2009.

[23]. Analyzing the Benefits of Mobile Enterprise Solutions Using the Example of Dispatching Processes. Botzenhardt, T. y Pousttchi, K. s.I. : IEEE, 2008. 7th International Conference on Mobile Business. págs. 260-269.

[24]. Mobile entertainment: model development and cross services study. Wong, C.C. y Hiew, P.L. s.I. : International Conference on Services Systems and Services Management, 2005. Proceedings of ICSSSM '05 . Vol. 2, págs. 1355- 1359. 
[25]. A Look at the Future of Networked and Mobile Entertainment. Zyda, M. 2007. 21st International Workshop on Principles of Advanced and Distributed Simulation, 2007. PADS '07. pág. 3.

[26]. LBS convergence agent for interoperability between LBS platforms. Kim, Jiho, Lee, Jungyu y Song, Ohyoung. 2009. 11th International Conference on Advanced Communication Technology, 2009. Vol. 1, págs. 863-866.

[27]. Security specification and implementation for mobile e-health services. Marti, R., Delgado, J. y Perramon, X. 2004. International IEEE Conference on eTechnology, e-Commerce and e-Service. págs. 241- 248.

[28]. WIRELESS/MOBILE E-COMMERCE:TECHNOLOGIES, APPLICATIONS, AND ISSUES. Tasarewich, P., Nickerson, R. y Warkentin, M. 2001. Proceedings Seventh Americas Conference on Information Systems. págs. 435-438.

[29]. The Impact of Mobile Technology on Business Processes Results from 5 Case Studies. Thumher, B. 2007. 2nd IEEE/IFIP International Workshop on BusinessDriven IT Management. págs. 108-109.

[30]. E-learning and Innovation Cultivation. Zheng, Yan, y otros. 2008. International Conference on Computer Science and Software Engineering. págs. 226-228.

[31]. Emerging Mobile Technologies and Issues. Subramanya, S.R. 2006. International Symposium on Collaborative Technologies and Systems. págs. 172172.

[32]. Goldsmith, Andrea. Wireless communications. s.l. : Cambridge University Press, 2005. ISBN: 9780521837163.

[33]. Dai, Lillian Lei. Proactive mobile wireless networks : an infrastructureless wireless network architecture for delay-sensitive applications. Massachusetts USA : Massachusetts Institute of Technology, 2008.

[34]. Association, IEEE Standard. IEEE 802.16: BROADBAND WIRELESS METROPOLITAN AREA NETWORKS (MANS). [En línea] [Citado el: 13 de 08 de 2010.] http://standards.ieee.org/getieee802/802.16.html.

[35]. WIRELESS BROADBAND ACCESS: WIMAX AND BEYOND - A Secure and Service-Oriented Network Control Framework for WiMAX Networks. Lu, Kejie, Qian, Yi y Chen, Hsiao-Hwa. 5, Mayo de 2007, Communications Magazine, IEEE, Vol. 45, págs. 124-130. 
[36]. A socio-economic analysis of WiMAX. 2009. International Conference on Application of Information and Communication Technologies. págs. 1-6. 9781424447398 .

[37]. Broadband radio access: LTE and LTE-advanced. Sawahashi, M., y otros. 2009. International Symposium on Intelligent Signal Processing and Communication Systems. págs. 224-227.

[38]. Association, IEEE Standards. IEEE 802.11 WIRELESS LOCAL AREA NETWORKS, The Working Group for WLAN Standards. [En línea] [Citado el: 13 de 08 de 2010.] http://www.ieee802.org/11/.

[39]. IEEE 802.15 WPAN Task Group 1 (TG1). IEEE 802.15 WPAN. [En línea] [Citado el: 13 de 07 de 2010.] http://www.ieee802.org/15/pub/TG1.html.

[40]. ZigBee Alliance. ZigBee Technical Documents . [En línea] [Citado el: 09 de 08 de 2010.] http://www.zigbee.org/Products/DownloadZigBeeTechnical Documents.aspx.

[41]. Association, IEEE Standards. IEEE 802.15 Working Group for WPAN. [En línea] [Citado el: 13 de Agosto de 2010.] http://www.ieee802.org/15/.

[42]. Union, International Telecommunications. Technology Glossary. [En línea] [Citado el: 13 de Agosto de 2010.] http://www.itu.int/telecomwt99/press_service/information_for_the_press/press_kit/backgrounders/techno logy_glossary-next-es.html.

[43]. UMTS Universal Mobile Telecommunications System: Development of Standards for the Third Generation. Samukic, Antun. 4, s.I. : IEEE TRANSACTIONS ON VEHICULAR TECHNOLOGY, 1998, Vol. 47.

[44]. Huish, P W. MOBILE BROADBAND SYSTEMS. s.I.: BT Laboratories, Martlesham Heath, IPSWICH, IP5 7RE, UK.

[45]. Union, International Telecommunications. Actividades PrincipalñesIMT2000. [En línea] [Citado el: 2010 de agosto de 13.] http://www.itu.int/aboutitu/annual_report/2000/key-imt2000-es.html.

[46]. CISCO. Channel Deployment Issues for 2.4-GHz 802.11 WLANs. [En línea] [Citado el: 6 de septiembre de 2010.] http://www.cisco.com/en/US/docs/wireless/technology/channel/deployment/g uide/Channel.html.

[47]. Engineers, Institute Of Electrical and Electronics. IEEE Std 802.11b-1999 (R2003) Local and metropolitan area networks-Specific requirements-. [En 
línea] [Citado el: 13 de Agosto de 2010.] http://standards.ieee.org/getieee802/download/802.11b-1999.pdf. ISBN 07381-1812-5.

[48]. IEEE Std 802.11a-1999(R2003) High-speed Physical Layer in the $5 \mathrm{GHz}$ Band. [En línea] [Citado el: 2010 de Agosto de 13.] http://standards.ieee.org /getieee802/download/802.11a-1999.pdf.

[49]. Phifer, Lisa. Wireless Privacy: An Oxymoron? Wi-fi Planet. [En línea] [Citado el: 2010 de agosto de 13 .] http://www.wifiplanet.com/columns/article.php/786641/Wireless-Privacy-An-Oxymoron.htm.

[50]. Cheung, Humphrey. FBI Teaches Lesson In How To Break Into Wi-Fi Networks . [En línea] [Citado el: 2010 de agosto de 13 .] http://www.informationweek.com/news/showArticle.jhtml?articleID=16050261 2.

[51]. Perillo, M. y Heinzelman, W. Wireless Sensor Network Protocols. Rochester, NY, USA : Department of Electrical and Computer Engineering.

[52]. A Simple and Efficient Method to Mitigate the Hot Spot Problem in Wireless Sensor Networks. Rivas, Helena and Voigt, Thiemo and Dunkels, Adam. Coimbra, Portugal.: Proceedings Performance Control in Wireless Sensor Networks, 2006. Vols. Rivas, Helena and Voigt, Thiemo and Dunkels, Adam.

[53]. Computer Science and Telecommunications Board (CSTB). Transport Protocols for Department of Defense Data Networks: Report to the Department of Defense and the National Bureau of Standards (1985) . [En línea] [Citado el: 2010 de Agosto de 13 .] http://www.nap.edu/openbook.php? record_id=10333\&page $=25$.

[54]. Packet Radio in AmateurService. Karn, P., Price, H. y Diersing, R. 3, s.I. : "IEEE JSAC, 1985, Vol. 3.

[55]. Jubin, J. and Torno, J.D. The DARPA Packet Radio project.

[56]. Mitch Waldrop, DARPA. DARPA and the Internet Revolution. [En línea] [Citado el: 13 de Agosto de 2010.] http://www.darpa.mil/Docs/Internet_ Development_200807180909255.pdf.

[57]. Accomplishments of the DARPA SURAN Program. Beyer, D.A. s.I. : IEEE, 1990. Military Communications Conference, 1990 MILCOM '90. Vol. 2, págs. 855862. 
[58]. Cleveland, Gary. Packet Radio: Applications for Libraries in Developing Countries. s.I. : UDT Series on Data Communication Technologies and Standards for Libraries, 1993.

[59]. Vittorio, Salvatore A. MicroElectroMechanical Systems (MEMS) . s.I.: ProQuest, 2001.

[60]. TinyOS Community Forum. An Open-source OS fot the Networked sensor regime. [En línea] [Citado el: 2010 de Agosto de 13.] http://www.tinyos.net /scoop/.

[61]. University of California. TinyDB: A Declarative Database for Sensor Networks. [En línea] [Citado el: 2010 de Agosto de 13.] http://telegraph.cs.berkeley.edu/tinydb/.

[62]. Adams, Jon. Smart Grid IEEE 802.15.4g - So Much Optionality. Smart Utility. [En línea] [Citado el: 22 de Octubre de 2010.] http://blogs.freescale.com/tag/smart-utility/.

[63]. Hsu, V.S. and Kahn, Joseph M. and Pister, Kristofer. Wireless Communications for Smart Dust. [En línea] EECS Department, University of California, Berkeley, Febrero de 1998. [Citado el: 21 de Abril de 2010.] http://www.eecs.berkeley.edu/Pubs/TechRpts/1998/3374.html.

[64]. SMART DUST, Autonomous sensing and communication in a cubic millimeter . [En línea] [Citado el: 12 de Mayo de 2010.] http://robotics.eecs.berkeley.edu/ pister/SmartDust/.

[65]. Smart Dust: communicating with a cubic-millimeter computer. Warneke, B., y otros. 1, Enero de 2001, Computer, Vol. 34, págs. 44-51. ISSN : 0018-9162.

[66]. A Taxonomy of Wireless Micro-Sensor Network Models. S. Tilak, N. AbuGhazaleh, and W.Heinzelman. 2, s.l.: ACM Mobile Computing and Communications Review (MC2R), 2002, Vol. 6. S. Tilak, N. Abu-Ghazaleh, and W..

[67]. Wireless sensor networks: a survey. Akyildiz, I.F., y otros. 38, s.I. : Elsevier Science B.V., 2002, Computer Networks, págs. 393-422.

[68]. Program, DOC / NOAA/ OAR / PMEL / VENTS. VENTS Program, exploring deep ocean ecosystems. [En línea] 1994. [Citado el: 26 de Agosto de 2010.] http://www.pmel.noaa.gov/vents/acoustics/sosus.html.

[69]. Pike, John. Integrated Undersea Surveillance System (IUSS). [En línea] Steven Aftergood, 1997. [Citado el: 26 de Agosto de 2010.] http://www.fas.org/irp/program/collect/iuss.htm. 
[70]. Kopetz, Hermann. Real-Time Systems Design Principles for Distributed Embedded Applications. Holanda : Kluwer Academic Publishers, 1997, 1997. 9780792398943.

[71]. Weiser, November 7, 1993. the world is not a desktop. [En línea] Perspectives article for ACM Interactions, 7 de Noviembre de 1993. [Citado el: 15 de Agosto de 2010.] http://www.ubiq.com/hypertext/weiser/ACMInteractions2.html.

[72]. Haenselmann, Thomas. Sensor Networks. [En línea] 2008. [Citado el: 14 de Septiembre de 2010.] http://pi4.informatik.unimannheim.de/ haensel/sn_book/.

[73]. Bode, Thomas. TinyDB \& COUGAR - Datenverarbeitung in Sensornetzen. Bonn, Germany : s.n., 2005.

[74]. Pfisterer, Dennis. Comprehensive Development Support for Wireless Sensor Networks . Luebeck : Institute für Telematics, 2007.

[75]. Baer, Martha. The Ultimate on-the-fly Network. [En línea] http://www.wired.com/wired/archive/11.12/network.html.

[76]. Wireless sensor networks for habitat monitoring. Mainwaring, A., Culler, D., Polastre, J., Szewczyk, R., and Anderson, J. [ed.] Proceedings of the 1st ACM international workshop on Wireless. s.I. : ACM Press, 2002. págs. 88-97.

[77]. Hardware design experiences in ZebraNet. Zhang, P., Sadler, C. M., Lyon, S. A., and Martonosi, M. Baltimore, Maryland, USA : SenSys'04, 2004.

[78]. Martonosi, Prof. Margaret. The Princeton ZebraNet Project: Sensor Networks for Wildlife Tracking. [En línea] Dept. of Electrical Engineering Princeton University, 2004. http://www.dtnrg.org/docs/workshopslides/martonosi-zebranet.pdf.

[79]. Monitoring Volcanic Eruptions with a Wireless Sensor Network. Geoffrey Werner-Allen, Jeff Johnson, Mario Ruiz, Jonathan Lees, and Matt Welsh. s.I. : Harvard University, 2005.

[80]. Harvard Sensor Networks Lab i. Volcano monitoring. [En línea] [Citado el: 12 de Mayo de 2010.] http://fiji.eecs.harvard.edu/.

[81]. Accenture. Pickberry Vineyard: Accenture Prototype Helps Improve Crop Management. [En línea] [Citado el: 12 de Junio de 2010.] http://www.accenture.com/Global/Services/Accenture_Technology_Labs/R_and _l/PickberryManagement.htm. 
[82]. A Multimodal Corpus Recorded in a Health Smart Home. A. Fleury, M. Vacher, F. Portet, P. Chahuara, N. Noury. Grenoble : s.n.

[83]. MarathonNet: adding value to large scale sport events - a connectivity analysis Full text. Pfisterer, Dennis, y otros. Niza, Francia: s.n., 2006. ISBN:159593-427-8 .

[84]. Praxiserfahrungen mit MarathonNet - Ein mobiles Sensornets im Sport. Hellbrueck, H., Lipphardt, M., Pfisterer, D., Ransom, S., and Fischer, S. 29, s.I. : PIK - Praxis der Informationsverarbeitung und Kommunikation, 2006, Vol. 4.

[85]. Towards a Zero-Configuration Wireless Sensor Network Architecture for Smart Buildings. Lars Schor, Philipp Sommer, Roger Wattenhofer. Berkeley, CA, USA : BuildSys'09, 3 de Noviembre de 2009.

[86]. Kim, Sukun. Wireless Sensor Networks for Structural Health Monitoring. Department of Electrical Engineering and Computer Sciences : s.n., 2005.

[87]. Kim, S., y otros. Structural Health Monitoring of the Golden Gate Bridge. [En línea] [Citado el: 28 de July de 2010.] http://www.cs.berkeley.edu/ binetude/ggb/.

[88]. Self-Healing Minefield . Global Security. [En línea] [Citado el: 2010 de Agosto de

http://www.globalsecurity.org/military/systems/munitions/shm.htm.

[89]. globalsecurity.org. http://www.globalsecurity.org/military/systems/munitions/shm.htm. [En línea] [Citado el: 15 de Julio de 2010.] http://www.globalsecurity.org/military/systems/munitions/shm.htm.

[90]. The daintiest dynamos. [Nuclear Microbatteries], 41(9):36-. Lal, Amit y Blanchard, Jake. 9, 2004, IEEE Spectrum, Vol. 41, págs. 36-41.

[91]. RF Monolithics, Inc. Sizing Solar Energy Harvesters for Wireless Sensor Networks. [En línea] 2010 . [Citado el: 16 de Septiembre de 2010.] http://www.rfm.com/products/apnotes/anm1002.pdf.

[92]. Parasitic Power Harvesting in Shoes. John Kymissis, Clyde Kendall, Joseph Paradiso,Neil Gershenfeld. [ed.] IEEE. Second IEEE International Conference on Wearable Computing : s.n., 1998.

[93]. A study of low level vibrations as a power source for wireless sensor nodes. Shad Roundy, Paul K. Wright, Jan Rabaey. Berkeley: Computer Communications, 2003, Vol. 26, págs. 1131-1144. 
[94]. Raju, Murugavel. White Paper. Energy Harvesting. [En línea] Texas Instruments, 2008. [Citado el: 16 de septiembre de 2010.] http://www.ti.com/corp/docs/landing/cc430/graphics/slyy018_20081031.pdf.

[95]. On the lifetime of wireless sensor networks. Dietrich, Isabel y Dressler, Falko. 1, s.I. : ACM, Febrero de 2009, ACM Trans. Sen. Netw, Vol. 5, pág. 39 pp.

[96]. Design and Implementation of Scalable Wireless Sensor Network for Structural Monitoring. Pakzad, Shamim N., y otros. 1, s.l. : March 1,, Journal of Infrastructure Systems, Vol. 14, págs. 89-101. ISSN 1076-0342/2008/1-89-101.

[97]. Farinaz Koushanfar, Miodrag Potkonjak, Alberto Sangiovanni-Vincentelli. FAULT TOLERANCE IN WIRELESS SENSOR NETWORKS. [En línea] 2002. [Citado el: 18 de Agosto de 2010.] http://citeseerx.ist.psu.edu/viewdoc/download?doi=10.1.1.87.1422\&rep=rep1\& type=pdf.

[98]. Optimal design of fault tolerant sensor networks. Hoblos, G, Staroswiecki, $\mathbf{M}$ y Aitouche, A. s.I. : IEEE International Conference on Control Applications, Anchorage, September 2000. págs. 467-472.

[99]. A new architecture for distributed sensor integration. D. Nadig, S.S. Iyengar. [ed.] Proceedings of IEEE Southeastcon'93. Charlotte : s.n., 1993.

[100]. Information Security Routing Protocol in the WSN. Wei, Li, Ming, Chen y Mingming, Li. 2009 . Fifth International Conference on Information Assurance and Security. Vol. 2, págs. 651-656.

[101]. Wagner, David. Security for Sensor Networks: Cryptography and Beyond. University of California at Berkeley. [En línea] [Citado el: 17 de septiembre de 2010.] http://www.cs.berkeley.edu/ daw/talks/SASN03.ppt.

[102]. WONG, J. L., FENG J., KIROVSKI D., and POTKONJAK M. Security in sensor networks: watermarking techniques. Wireless Sensor Networks. Norwell, MA : Kluwer Academic Publishers, 2004, págs. 305-323.

[103]. Earthquake Shakes Twitter Users: Real-time Event Detection by Social Sensors. Sakaki, Takeshi, Okazaki, Makoto y Matsuo, Yutaka. Raleigh, North Carolina : WWW2010, 2010.

[104]. Energy-Aware Wireless Microsensor Networks. V. Raghunathan, C. Schurgers, S. Park, and M. B. Srivastava. 19, s.l.: IEEE Signal Processing Magazine, 2002, págs. 40-50. 
[105]. McCusker, Kealan. CRYPTOGRAPHIC KEY DISTRIBUTION IN WIRELESS SENSOR NETWORKS: A HARDWARE PERSPECTIVE. Tesis Doctorado en Dublin City University. [En línea] 2008. [Citado el: 2010 de Septiembre de 18.] http://doras.dcu.ie/110/1/mccusker_kealan_2008.pdf.

[106]. Agent Platform for Wireless Sensor Network with Support for Cryptographic Protocols. Peter Pecho, Frantisek Zboril jr.,Martin Drahansky, Petr Hanacek. 5, s.l. : Universal Computer Science, 2009, Journal of Universal Computer Science, Vol. 15.

[107]. Laura, De Giusti. Tesis Doctoral: Mapping sobre Arquitecturas Heterogéneas. La Plata : Universidad Nacional de La Plata, 2008.

[108]. Intel . Inside Sensor Network Technology. Intel Research Area Web Site. [En línea] 2005. [Citado el: 12 de Febrero de 2010.] http://www.intel.com/research/exploratory/wireless_promise.htm.

[109]. A survey of applications of wireless sensors and wireless sensor networks. Arampatzis, T., Lygeros, J. y Manesis, S. Cyprus: s.n., 2005. Mediterranean Conference on Control and Automation. págs. 719-724.

[110]. Wireless sensor networks powered by ambient energy harvesting (WSNHEAP) - Survey and challenges. Seah, W.K.G., Eu, Zhi Ang y Tan, Hwee-Pink. 2009 . 1st International Conference on Wireless Communication, Vehicular Technology, Information Theory and Aerospace \& Electronic Systems Technology. págs. 1-5.

[111]. System software techniques for low-power operation in wireless sensor networks. Dutta, P. y Culler, D. San Jose, CA: s.n., 2005. Proc. Int'I Conf. Computer Aided Design (ICCAD'05). págs. 925-932.

[112]. Young, Joel K. A Practical Guide to Battery Technologies for Wireless Sensor Networking. [En línea] 2008. [Citado el: 20 de septiembre de 2010.] http://www.sensorsmag.com/networking-communications/batteries/a-practicalguide-battery-technologies-wireless-sensor-netwo-1499.

[113]. Energy-efficient characterization of solar panel-supercapacitors systems for energy-harvesting aware wireless sensor nodes. da Cunha, A.B. y da Silva, D.C. 2009. IEEE 20th International Symposium on Personal, Indoor and Mobile Radio Communications. págs. 2275-2279.

[114]. Design and Power Management of Energy Harvesting Embedded Systems. Raghunathan, V. y Chou, P.H. 2006. Proceedings of the 2006 International Symposium on Low Power Electronics and Design. págs. 369-374. 
[115]. Smart applications for energy harvested WSNs. Prabhakar, T.V., y otros. 2010. Proc. Second International Conference on Communication Systems and Networks (COMSNETS). págs. 1-7.

[116]. Ultra low-power radio design for wireless sensor networks. Enz, C., Scolari, N. y Yodprasit, U. 2005. Proceedings 2005 IEEE International Workshop on Radio-Frequency Integration Technology. pág. 2005 IEEE International Workshop on.

[117]. Aluko, Mobolaji E. Why NCC Should Allow ISPs To Use ISM Bands Despite ITU. [En línea] [Citado el: 19 de agosto de 2010.] http://www.nigerdeltacongress.com/warticles/why_ncc_should_allow_isps_to_ use.htm.

[118]. McLarnon, Barry. VHF/UHF/Microwave Radio Propagation: A Primer for Digital Experimenters. [En línea] [Citado el: 2010 de mayo de 16.] http://www.tapr.org/ve3jf.dcc97.html.

[119]. Dynamic power management in wireless sensor networks. Sinha, A. y Chandrakasan, A. 2, 2001, IEEE Design \& Test of Computers, Vol. 18, págs. 6274.

[120]. TI. 2.4 GHz IEEE 802.15.4 ZigBee low cost, low power System-on-Chip (SoC) solution (rev. 2.1). 2006.

[121]. Application-oriented wireless sensor network communication protocols and hardware platforms: A survey. Pei, Zhongmin, y otros. 2008. IEEE International Conference on Industrial Technology. págs. 1-6.

[122]. Field programmable gate arrays. Verma, H. 4, s.I. : IEEE, 1999, Potentials, Vol. 18, págs. 34-36.

[123]. Field programmable gate arrays in space. Leon, A.F. 4, s.I. : IEEE, 2003, Instrumentation \& Measurement Magazine, Vol. 6, págs. 42- 48.

[124]. A conceptual framework for ASIC design. Leung, S.S., Fisher, P.D. y Shanblatt, M.A. 7, s.I. : Proceedings of the IEEE, 1988, Vol. 76, págs. 745-755.

[125]. Intel. [En línea] Julio de 1999. [Citado el: 15 de Mayo de 2010.] http://www.datasheetcatalog.org/datasheets/90/361041_DS.pdf.

[126]. Texas Instruments. MSP430x1xx Family. [En línea] 2006. [Citado el: 2010 de Abril de 13.] http://focus.ti.com/lit/ug/slau049f/slau049f.pdf. 
[127]. Jennic Wireless Microcontrollers. JN5139 Wireless Microcontroller (IEEE802.15.4 and JenNet) . [En línea] [Citado el: 15 de Junio de 2010.] http://www.jennic.com/products/wireless_microcontrollers/jn5139.

[128]. Holger, K. y Willig, Andreas. Protocols and Architectures for Wireless Sensor Networks. s.I. : John Wiley \& Sons, 2005. ISBN-13 978-0-470-09510-2.

[129]. Optimizing RF front ends for low power. Baltus, P.G.M. y Dekker, R. s.I. : IEEE, 2000. Proceedings of the IEEE . Vol. 88 (10, págs. 1546 - 1559 . 0018-9219.

[130]. Wu, Shih-Lin, [ed.]. Wireless Ad Hoc Networking: Personal-Area, LocalArea, and the Sensory-Area Networks. 2007. 780849392542 .

[131]. Ilyas, Mohammad, [ed.]. The Handbook of Ad Hoc Wireless Networks . 2002.

[132]. Wu, X. Chen and J. The Handbook of Ad Hoc Wireless Networks. s.I. : CRC, 2003.

[133]. Perkins, Charles E. Ad Hoc Networking. s.l. : Addison-Wesley Professional, 2001. 978-0201309768.

[134]. Stojmenovic, Ivan, [ed.]. Handbook of Wireless Networks and Mobile Computing. s.I. : Wiley. ISBN: 978-0-471-41902-0.

[135]. Network planning in wireless ad hoc networks: a cross-Layer approach. Wu, Y., y otros. 1, 2005, Vol. 23. ISSN: 0733-8716.

[136]. Barkai, David. Peer-to-Peer Computing: Technologies for Sharing and Collaborating on the Net. s.I. : Intel Press, 2002.

[137]. Cockayne, William R. y Zyda, Michael. Mobile Agent. s.I. : Prentice Hall, 1998. ISBN-10: 0138582424.

[138]. Energy optimization in multihop wireless embedded and sensor networks. Shelby, Z., Pomalaza-Raez, C. y Haapola, J. 2004. 15th IEEE International Symposium on Personal, Indoor and Mobile Radio Communications. Vol. 1, págs. 221- 225 .

[139]. Coping with unreliable channels: Efficient link estimation for low-power wireless sensor networks. Meier, A., y otros. 2008. 5th International Conference on Networked Sensing Systems. págs. 19-26.

[140]. Reliability analysis of Wireless Sensor Network. Purohit, N., Varadwaj, P. y Tokekar, S. 2008. 16th IEEE International Conference on Networks ICON 2008. págs. 1-6. 
[141]. Deploying Multiple Sinks in Multi-hop Wireless Sensor Networks. Vincze, Zoltan, Vida, Rolland y Vidacs, Attila. 2007. IEEE International Conference on Pervasive Services. págs. 55-63.

[142]. A Taxonomy of Wireless Micro-Sensor Network Models. Tilak, S., AbuGhazaleh, N. y Heinzelman, W. 2, 2002, obile Computing and Communications Review, Vol. 1, págs. 1-8.

[143]. A survey on wireless multimedia sensor networks. Akyildiz, lan F., Melodia, Tommaso y Chowdhury, Kaushik R. 51 , s.I. : Elsevier B.V., 2007, Computer Networks, Vol. Computer Networks, págs. 921-960.

[144]. QoS Support in Wireless Sensor Networks: A Survey. Chen, D. y Varshney, P. Las Vegas, Nevada, USA: s.n., 2004. Proc. of the 2004 International Conference on Wireless Networks (ICWN 2004).

[145]. Scalability Analysis for Wireless Sensor Networks Routing Protocols. Alazzawi, L.K., Elkateeb, A.M. y Ramesh, A. 2008. 22nd International Conference on Advanced Information Networking and Applications - Workshops, 2008. AINAW 2008. págs. 139-144.

[146]. Middleware Challenges for Wireless Sensor Networks. Kay Romer, Oliver Kasten, Friedemann Mattern. 2, 2002, Mobile Computing and Communications Review, Vol. 6, págs. 59-61.

[147]. Middleware: Middleware Challenges and Approaches for Wireless Sensor Networks. Hadim, Salem y Mohamed, Nader. 3, s.I. : IEEE Computer Society, Marzo de 2006, IEEE DISTRIBUTED SYSTEMS ONLINE 1541-4922, Vol. 7, págs. 123.

[148]. TAG: a Tiny AGgregation Service for Ad-Hoc Sensor Networks. Samuel Madden, Michael J. Franklin, Joseph Hellerstein, Wei Hong. SI, Berkeley : ACM, 2002, Vol. 36, págs. 131-146 . ISSN:0163-5980 .

[149]. Noiseless Coding of Correlated Information Sources. Slepian, J. y J.Wolf. [ed.] IEEE. 4, 1973, IEEE Transactions on Information Theory, Vol. 19, págs. 471480.

[150]. Bradshaw, Jeffrey M., [ed.]. Software Agents. J. Bradshaw. s.I. : AAAI Press/MIT Press, 1996. 0262522349.

[151]. Swarm intelligence based key generation for text encryption in cellular networks. Sreelaja, N.K. y Vijayalakshmi Pai, G.A. Sreelaja, N.K.; Vijayalakshmi Pai, G.A. : s.n., 2008. Communication Systems Software and Middleware and Workshops, 2008. págs. 622-629. 
[152]. A Distributed Location Service for MANET Using Swarm Intelligence. Gajurel, S. y Heiferling, M. s.I. : Mobile WiMAX Symposium, . MWS '09. IEEE, 2009. págs. 220-225.

[153]. SwarmNet project. [En línea] [Citado el: 19 de 08 de 2009.] http://www.swarmnet.de/.

[154]. A New Authentication Method with Distributed Hash Table for P2P Network. Takeda, A., y otros. Okinawa : Advanced Information Networking and Applications - Workshops, 2008. ISBN: 978-0-7695-3096-3.

[155]. A Distributed Hash Table-based Approach for Providing a File System Neutral, Robust and Resilient Storage Infrastructure. Parissis, G. y Apostolopoulos, T. Aveiro : Next Generation Internet Networks, 2009. NGI '09, 2009. ISBN: 978-1-4244-4244-7.

[156]. A distributed hash table for computational grids. Riley, C. y Scheideler, C. Baltimore : Parallel and Distributed Processing Symposium, 2004. Proceedings. 18th International, 2004.

[157]. The design of topology-aware overlay networks for ubiquitous applications. Chiu, Chuan-Feng, y otros. [ed.] 2009 Joint Conferences on Pervasive Computing (JCPC). Tamsui, Taipei : s.n., 2009. págs. $229-234$. ISBN: 978-1-4244-5227-9.

[158]. Fisheye: Topology aware choice of peers for overlay networks. Milic, D. Braun, T. Zurich : Conference on Local Computer Networks, 2009. LCN 2009. IEEE 34th , 2009. ISBN: 978-1-4244-4488-5 .

[159]. Hoang, Xuantung y Lee, Younghee. An efficient scheme for reducing overhead in data-centric storage sensor networks. S.I. : IEEE Communications Society, 2009. ISSN:1089-7798 .

[160]. Data-centric cooperative storage in wireless sensor network. Awad, A., Germany, R. y Dressler, F. Bratislava : 2nd International Symposium on Applied Sciences in Biomedical and Communication Technologies. ISABEL 2009., 2009. págs. 1-6. ISBN: 978-1-4244-4640-7.

[161]. The Analysis of Publish/Subscribe Systems over Mobile Wireless Ad Hoc Networks. Pongthawornkamol, T., Nahrstedt, K. y Wang, Guijun. Philadelphia : s.n., 2007. págs. 1-8. ISBN: 978-1-4244-1024-8.

[162]. Bouncing Tracks in Sensor Networks. Li, Zhigang, y otros. Melbourne : 14th IEEE International Conference on Parallel and Distributed Systems, 2008. ICPADS '08. , 2008. ISBN: 978-0-7695-3434-3 . 
[163]. Giuseppe Amato, Stefano Chessa, Francesco Furfari, Stefano Lenzi, Claudio Vairo. Towards Data Management in the Sensor Web: the MaD-WiSe System . [En línea] [Citado el: 12 de Septiembre de 2010.] http://ercimnews.ercim.eu/en76/special/towards-data-management-in-the-sensor-web-themad-wise-system.

[164]. Performance evaluation of databases integration in wireless sensor networks. Patiño, D.C.R., Carranza, D.A.O. y de J Montoya Canola, A. 2009. IEEE Latin-American Conference on Communications LATINCOM '09. págs. 1-6.

[165]. Andrew S. Tanenbaum, David J. Wetherall. Computer Networks. s.I. : Prentice Hall. , 2010. 0132126958.

[166]. Architectures for heterogeneous wireless sensor networks. Durresi, A. 2005. IEEE 16th International Symposium on Personal, Indoor and Mobile Radio Communications . Vol. 2, págs. 1289-1296.

[167]. Real-world implementation of the location stack: the universal location framework, Graumann, D., y otros. 2003. Proceedings Fifth IEEE Workshop on Mobile Computing Systems and Applications. págs. 122- 128.

[168]. Coulouris, G., Dollimore, J. y Kindberg, T. Dsitributed Systems, Concepts and Design. Harlow : Pearsons Education, 2001. ISBN: 0201-61918-0.

[169]. Tanenbaum, A. Organización de Computadoras. Un Enfoque Estructurado. s.l. : Prentice Hall. 9701703995.

[170]. Simulation Tools for Wireless Sensor Networks. Egea-López, E., y otros. 2005. Summer Simulation Multiconference - SPECTS 2005. págs. 1-9.

[171]. Wainer, Gabriel A. Discrete-Event Modeling and Simulation. A Practitioner's Approach. s.I. : CRC Press, 2009. ISBN: 978-1-4200-5337-1.

[172]. Survey of Wireless Sensor Networks Simulation Tools for Demanding Applications. Korkalainen, M., y otros. 2009. Fifth International Conference on Networking and Services ICNS '09. págs. 102-106.

[173]. Simulation Tools for Wireless Sensor Networks. Egea-López, E., y otros. Philadelphia : s.n., 2005. International Symposium on Performance Evaluation of Computer and Telecommunication Systems (SPECTS05). ISBN:1-56555-300-4.

[174]. Shawn: The fast, highly customizable sensor network simulator. Fekete, S.P., y otros. Braunschweig : Networked Sensing Systems, 2007. INSS '07. Fourth International Conference., 2007. págs. 299 - 299 . ISBN: 1-4244-1231-5. 
[175]. NetTopo: A Framework of Simulation and Visualization for Wireless Sensor Networks. Shua, Lei, y otros. s.I. : Elsevier B.V., 2010, Ad Hoc Networks, Vols. Manuscito aprobado, en impresión.

[176]. Classification of Analysis Techniques for Wireless Sensor Networks. Prasad, Vibha y Son, Sang H. Braunschweig: s.n., 2007. Networked Sensing Systems, 2007. INSS '07. Fourth International Conference on . págs. 93 - 97. ISBN: 1-42441231-5.

[177]. An analysis of a large scale habitat monitoring application. Szewczyk, R., Mainwaring, A., Polastre, J., Anderson, J., and Culler, D. 2004. Proceedings of the 2nd international conference on Embedded networked sensor system, SenSys 2004.

[178]. Sensor: the atomic computing particle. Vijay, Kumar. 4, s.I. : SIGMOD Rec., 2003, Vol. 32, págs. 16-21.

[179]. Sizing up smart dust. Gorder, Pam Frost. 6, Computing in Science \& Engineering, Vol. 5, págs. 6-9. ISSN:1521-9615.

[180]. On the optimal number of smart dust particles. Khan, S.U. y Hamid, M.S. [ed.] 2003. INMIC 2003. 7th International Multi Topic Conference. 2003. págs. $472-475$.

[181]. SensorSim: A simulation framework for sensor networks. Park, S., Savvides, A. y Srivastava, M. Boston : ACM, 2000. Proceedings of the 3rd ACM international workshop on Modeling, analysis and simulation of wireless and mobile systems. págs. 104 - 111 . ISBN:1-58113-304-9 .

[182]. ns2. ns-2. [En línea] [Citado el: 10 de Agosto de 2010.] http://nsnam.isi.edu/nsnam/.

[183]. J-Sim: a simulation and emulation environment for wireless sensor networks. Sobeih, A., y otros. [ed.] IEEE. 4, 2006, Wireless Communications, Vol. 13, págs. 104-119.

[184]. Shawn simulator. [En línea] [Citado el: 15 de Marzo de 2010.] http://shawn.sf.net.

[185]. A Visualization and Analysis Tool for NS-2 Wireless Simulations: iNSpect. Kurkowski, S., y otros. Washington D.C.: IEEE Computer Society, 2005. Proceedings of the 13th IEEE international Symposium on Modeling, Analysis, and Simulation of Computer and Telecommunication Systems. MASCOTS . págs. 503-506. 
[186]. G. Chen, J., M.J. Branch, L.Z. Pflug, and B. Szymanski. SENSE: A Sensor Network Simulator. [ed.] B. Szymanksi and B. Yener. 2004. págs. 249-267. en Advances in Pervasive Computing \& Networking.

[187]. Naval Research Laboratory, USA. NRL Extensions to NS-2. Network and Communication System Branch. [En línea] 15 de 09 de 2010. http://cs.itd.nrl.navy.mil/work/proteantools/ns2extensions.php.

[188]. SensorSim: a simulation framework for sensor networks. Park, S., Savvides, A. y Srivastava, M. s.I. : ACM, 2000. Proceedings of the 3rd ACM international workshop on Modeling, analysis and simulation of wireless and mobile systems (MSWIM '00).

[189]. Institute for Sofware Integrated Systems. Prowler: Probabilistic Wireless Network Simulator. [En línea] [Citado el: 11 de Mayo de 2010.] http://www.isis.vanderbilt.edu/Projects/nest/prowler/.

[190]. Institute for Software Integrated Systems. JProwler. [En línea] [Citado el: 11 Mayo de 2010.] http://w3.isis.vanderbilt.edu/projects/nest/JProwler/index.html.

[191]. Using the OMNeT++ discrete event simulation system in education. Varga, A. 4, s.I. : IEEE Education Society, 1999, IEEE Transactions on Education, Vol. 44, pág. 11.

[192]. Simulation of Large-Scale Sensor Networks Using GTSNetS. EIMoustapha Ould-Ahmed-Vall, George F. Riley, Bonnie S. Heck, Dheeraj Reddy. 2005. págs. 211-218. 13th IEEE International Symposium on Modeling, Analysis, and Simulation of Computer and Telecommunication Systems.

[193]. J-Sim: a simulation and emulation environment for wireless sensor networks. Sobeih, A., y otros. 4, s.I. : IEEE , 2006, Wireless Communications, Vol. 13, págs. 104-119.

[194]. Sobeih, Ahmed y Hou, Jennifer C. A Simulation Framework for Sensor Networks in J-Sim. 2003.

[195]. SENS: a sensor, environment and network simulator. Sundresh, S., Kim, Wooyoung y Agha, G. 2004. Proceedings. 37th Annual Simulation Symposium. págs. 221- 228.

[196]. TOSSIM: Accurate and scalable simulation ofentire TinyOS applications. P. Levis, N. Lee, M. Welsh, and D. Culler. Los Angeles : s.n., 2003. Proc. Int'I Conf. Embedded Networked Sensor Systems (SenSys'03). págs. 126-137. 
[197]. Jason Hill, Robert Szewczyk, Alec Woo, Seth Hollar, David Culler, Kristofer Pister. System architecture directions for networked sensors. [En línea] [Citado el: 13 de julio de 2010.] http://www.tinyos.net/papers/tos.pdf.

[198]. Philip Levis, Sam Madden, Joseph Polastre, Robert Szewczyk, Kamin Whitehouse, Alec Woo, David Gay, Jason Hill, Matt Welsh, Eric Brewer, David Culler. TinyOS: An Operating System for Wireless Sensor Networks. s.I.: W Weber, J Rabaey, and E Aarts, 2005. En el libro "Ambient Intelligence".

[199]. Demo Abstract: TinyOS 2.0. Group, The TinyOS 2.x Working. 2005. Proceedings of the 3rd international conference on Embedded networked sensor systems- SenSys '05.

[200]. Simulating the power consumption of large-scale sensor network applications. Shnayder, V., y otros. s.I. : ACM, 2004. Proceedings of the 2nd international Conference on Embedded Networked Sensor Systems-SenSys '04. 188-200.

[201]. Shawn. [En línea] [Citado el: 15 de Mayo de 2009.] http://shawnwiki.coalesenses.com.

[202]. Shawn. Shawn Wiki. [En línea] [Citado el: 19 de Octubre de 2009.] http://shawn.sourceforge.net/.

[203]. Ganesan, D., y otros. Complex behavior at scale: An experimental study of low-power wireless sensor networks. . s.I. : Tech. rep., UCLA, 2002.

[204]. Radio irregularity problem in wireless sensor networks: New experimental results. Ababneh, N. Sarnoff Symposium, 2009. SARNOFF '09. : IEEE , 2009. págs. 1-5.

[205]. Stallings, William. Data and computer communications. s.I. : Prentice Hall, 2007. 0132433109.

[206]. George Coulouris, Jean Dollimore, Tim Kindberg. Distributed Systems: Concepts and Design. s.I. : Addison-Wesley, 2005. 0321263545.

[207]. GNU. GCC, the GNU Compiler Collection - GNU Project - Free Software Foundation (FSF). [En línea] [Citado el: 23 de Junio de 2010.] http://gcc.gnu.org/.

[208]. GMBH, Coalesenses. Wireless Sensor Networks - Hardware and Software Solutions by Coalesenses $\mathrm{GmbH}$ - iShell. [En línea] [Citado el: 15 de Mayo de 2010.] http://www.coalesenses.com/index.php?page=ishell.

[209]. - . Operating and Networking Firmware. [En línea] [Citado el: 15 de Omayo de 2009.] http://www.coalesenses.com/index.php?page=software-system. 
[210]. Eclipse. Eclipse IDE for C/C++ Developers. [En línea] [Citado el: 15 de 08 de 2010.] http://www.eclipse.org.

[211]. A Survey of Simulators, Emulators and Testbeds for Wireless Sensor Networks. Imran, Muhammad, Said, Abas Md y Hasbullah, Halabi. s.I. : IEEE, 2010. 978-1-4244-6716-7.

[212]. Coalesenses. Download iSense Firmware. [En línea] [Citado el: 10 de Abril de 2010.] http://www.coalesenses.com/index.php?page=isense-firmware.

[213]. Twitter. [En línea] 12 de 02 de 2010. [Citado el: 20 de Febrero de 2010.] http://twitter.com/.

[214]. Sarah Milstein, Abdur Chowdhury, Gregor Hochmuth, Ben Lorica, Roger Magoulas. Twitter and the Micro-Messaging Revolution. [En línea] 2008. [Citado el: 15 de Mayo de 2010.] http://www.weigend.com/files/ teaching/haas/2009/readings/OReillyTwitterReport2008.

[215]. Sakaki, Takeshi, Okazaki, Makoto y Matsuo, Yutaka. Earthquake Shakes Twitter Users: Real-time Event Detection by Social Sensors. [En línea] 2010. [Citado el: 15 de Agosto de 2010.] http://ymatsuo.com/papers/www2010.pdf.

[216]. Internet Engineering Task Force (IETF). Request for Comments: 5849: The OAuth 1.0 Protocol. [En línea] aBRIL de 2010. [Citado el: 15 de Agosto de 2010.] http://tools.ietf.org/html/rfc5849.

[217]. Liechti, Chris. pySerial v2.5 documentation. [En línea] 2010. [Citado el: 13 de Junio de 2010.] http://pyserial.sourceforge.net/examples.html\#miniterm. 


\section{Apéndice A: Archivo}

\section{configuración simulación de localización}

create_normal name=normal_multiplier mean $=1$ stddev $=0.1$

create_randomized_distance_estimate name=normal_dist multiplier=normal_multiplier chop_low=0 resample_chopped=false

create_uniform name=uniform_multiplier lower=.9 upper=1.1

create_randomized_distance_estimate name=uniform_dist multiplier=uniform_multiplier chop_low $=0$ resample_chopped=false prepare_world edge_model=list comm_model=disk_graph range $=70$ transm_model=stats_chain

chain_transm_model name=random_drop_chain probability $=0.0$ chain_transm_model name=reliable

logging_reconfigure action=set_level logger=processors.localization level=info logging_reconfigure action=set_additivity logger=user additivity=false

loc_est_dist=normal_dist

loc_dist_algo=sum_dist

loc_pos_algo=lateration

loc_ref_algo=iterative_lateration

loc_check_residue=true

loc_floodlimit $=4$

loc_idle_time $=5$

loc_idle_shutdown_time $=20$

loc_startup_anchor_frac $=0$

loc_eucl_col_check_std=lax

loc_eucl_col_check_nv=lax

loc_eucl_col_check_cn=strict 


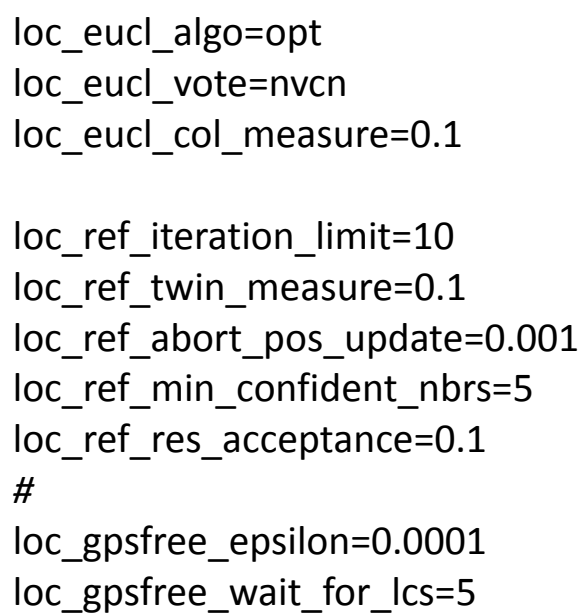

\#\# Si el alcance radial supuesto es de $\mathbf{2 0 0} \mathbf{m}$ de cada uno

\#\#de los nodos iSense

\#\#\# El espacio de 500x500 representaria 70/200=500/x aprox $2 \mathrm{Km}$ cuadrados

rect_world width $=500$ height $=500$ count $=200$ processors=localization

\# anchor_placement: outer_grid, inner_grid, random

\# placed_anchor_cnt: $>=0$

localization_anchor_placement anchor_placement=outer_grid placed_anchor_cnt $=12$

\# start simulation

simulation max_iterations $=50$

\# ps_out: optional filename of postscript-output of the topology \# Is_out: optional filename to write data in csv-style \# type: required, if Is_out is set. choices are 'create' and 'append'. localization_evaluation loc_ps_out=topology.ps loc_Is_out=test.csv loc_ls_type=create 


\title{
Apéndice B. Resultado de la simulación de localización
}

\author{
init_apps: init_topology(sc); init_examples(sc); init_localization(sc); \\ init_vis(sc); init_reading(sc); \\ init_topology_elevation \\ init_topology_generator \\ init_topology_node_gen \\ init_topology_node_mod \\ init_topology_point_gen \\ init_topology_point_mod \\ init_topology_topology \\ Initialising examples \\ Simulation: Running task 'create_normal' \\ Simulation: Task done 'create_normal' \\ Simulation: Running task 'create_randomized_distance_estimate' \\ Simulation: Task done 'create_randomized_distance_estimate' \\ Simulation: Running task 'create_uniform' \\ added uniform_multiplier \\ Simulation: Task done 'create_uniform' \\ Simulation: Running task 'create_randomized_distance_estimate' \\ Simulation: Task done 'create_randomized_distance_estimate' \\ Simulation: Running task 'prepare_world' \\ DiskGraphModel: Transmission range set to [70] \\ Simulation: Task done 'prepare_world' \\ Simulation: Running task 'chain_transm_model' \\ random_drop: Initialised. Drop probability is 0 \\ Simulation: Task done 'chain_transm_model' \\ Simulation: Running task 'chāin_transm_model' \\ Simulation: Task done 'chain_transm_model' \\ Simulation: Running task 'logging_reconfigure' \\ Simulation: Task done 'logging_reconfigure' \\ Simulation: Running task 'logging_reconfigure' \\ Simulation: Task done 'logging_reconfigure' \\ Simulation: Running task 'rect_world' \\ Simulation: Task done 'rect_world' \\ Simulation: Running task 'localization_anchor_placement' \\ Simulation: Task done 'localization_anchor_placement' \\ Simulation: Running task 'simulation' \\ LocalizationLaterationModule: No neighbors found
}


LocalizationLaterationModule: No neighbors found LocalizationLaterationModule: No neighbors found LocalizationLaterationModule: No neighbors found LocalizationLaterationModule: No neighbors found LocalizationLaterationModule: No neighbors found LocalizationLaterationModule: No neighbors found LocalizationLaterationModule: No neighbors found LocalizationLaterationModule: No neighbors found DONE ITERATION 0

Hasta la iteración 19 el resultado de la simulación repite los resultados de iteración 0

\section{BEGIN ITERATION 20}

LocalizationLaterationModule: No neighbors found LocalizationLaterationModule: No neighbors found LocalizationLaterationModule: No neighbors found LocalizationLaterationModule: No neighbors found LocalizationLaterationModule: No neighbors found LocalizationLaterationModule: No neighbors found LocalizationLaterationModule: No neighbors found LocalizationLaterationModule: No neighbors found LocalizationLaterationModule: No neighbors found DONE ITERATION 20

[ 189 active, 0 sleeping, 11 inactive ]

BEGIN ITERATION 21

LocalizationLaterationModule: No neighbors found LocalizationLaterationModule: No neighbors found LocalizationLaterationModule: No neighbors found LocalizationLaterationModule: No neighbors found LocalizationLaterationModule: No neighbors found LocalizationLaterationModule: No neighbors found LocalizationLaterationModule: No neighbors found LocalizationLaterationModule: No neighbors found LocalizationLaterationModule: No neighbors found DONE ITERATION 21

[ 132 active, 0 sleeping, 68 inactive ]

\section{BEGIN ITERATION 22}

LocalizationLaterationModule: No neighbors found LocalizationLaterationModule: No neighbors found LocalizationLaterationModule: No neighbors found LocalizationLaterationModule: No neighbors found LocalizationLaterationModule: No neighbors found LocalizationLaterationModule: No neighbors found 
LocalizationLaterationModule: No neighbors found LocalizationLaterationModule: No neighbors found LocalizationLaterationModule: No neighbors found DONE ITERATION 22

[ 68 active, 0 sleeping, 132 inactive ]

\section{BEGIN ITERATION 23}

LocalizationLaterationModule: No neighbors found LocalizationLaterationModule: No neighbors found LocalizationLaterationModule: No neighbors found LocalizationLaterationModule: No neighbors found LocalizationLaterationModule: No neighbors found LocalizationLaterationModule: No neighbors found LocalizationLaterationModule: No neighbors found LocalizationLaterationModule: No neighbors found LocalizationLaterationModule: No neighbors found DONE ITERATION 23

[ 28 active, 0 sleeping, 172 inactive ]

\section{BEGIN ITERATION 24}

LocalizationLaterationModule: No neighbors found LocalizationLaterationModule: No neighbors found LocalizationLaterationModule: No neighbors found LocalizationLaterationModule: No neighbors found LocalizationLaterationModule: No neighbors found LocalizationLaterationModule: No neighbors found LocalizationLaterationModule: No neighbors found LocalizationLaterationModule: No neighbors found LocalizationLaterationModule: No neighbors found DONE ITERATION 24

[ 13 active, 0 sleeping, 187 inactive ]

\section{BEGIN ITERATION 25}

LocalizationLaterationModule: No neighbors found LocalizationLaterationModule: No neighbors found LocalizationLaterationModule: No neighbors found LocalizationLaterationModule: No neighbors found LocalizationLaterationModule: No neighbors found LocalizationLaterationModule: No neighbors found LocalizationLaterationModule: No neighbors found LocalizationLaterationModule: No neighbors found LocalizationLaterationModule: No neighbors found DONE ITERATION 25

[ 10 active, 0 sleeping, 190 inactive ] 
LocalizationLaterationModule: No neighbors found LocalizationLaterationModule: No neighbors found LocalizationLaterationModule: No neighbors found LocalizationLaterationModule: No neighbors found LocalizationLaterationModule: No neighbors found LocalizationLaterationModule: No neighbors found LocalizationLaterationModule: No neighbors found LocalizationLaterationModule: No neighbors found LocalizationLaterationModule: No neighbors found DONE ITERATION 26

[ 9 active, 0 sleeping, 191 inactive ]

Repite resultados hasta iteración 49 BEGIN ITERATION 49

LocalizationLaterationModule: No neighbors found LocalizationLaterationModule: No neighbors found LocalizationLaterationModule: No neighbors found LocalizationLaterationModule: No neighbors found LocalizationLaterationModule: No neighbors found LocalizationLaterationModule: No neighbors found LocalizationLaterationModule: No neighbors found LocalizationLaterationModule: No neighbors found LocalizationLaterationModule: No neighbors found DONE ITERATION 49

[ 9 active, 0 sleeping, 191 inactive ]

Simulation: Task done 'simulation'

Simulation: Running task 'localization_evaluation'

Distance algorithm: sum_dist

Position algorithm: lateration

Refinement algorithm: iterative_lateration

191 nodes know their position, 0 are still unknowning. That's a coverage of $100 \%$.

Relative anchor distances (real): mean is 0.0222764 , std-dev is 0.0975153 ( from -0.21541 to 0.791624 )

Relative anchor distances (comm_range): mean is 0.0520071 , std-dev is 0.230621 ( from -0.434899 to $1.4 \overline{7} 764$ )

Average absolute distance from real pos: 10.1156 ( $14.4508 \%$ of communication range ) ( from 0.41984 to 55.8825 )

Average number of neighbors per node is 11.2251 ( from 3 to 25 )

Simulation: Task done 'localization_evaluation'

random_drop: 4852 msgs. to be sent, 0 of them dropped_, $0 \%$ dropped_; desired probabilty was: $0 \%$ 


\section{Apéndice C: Código programa}

\section{instalado en nodos}

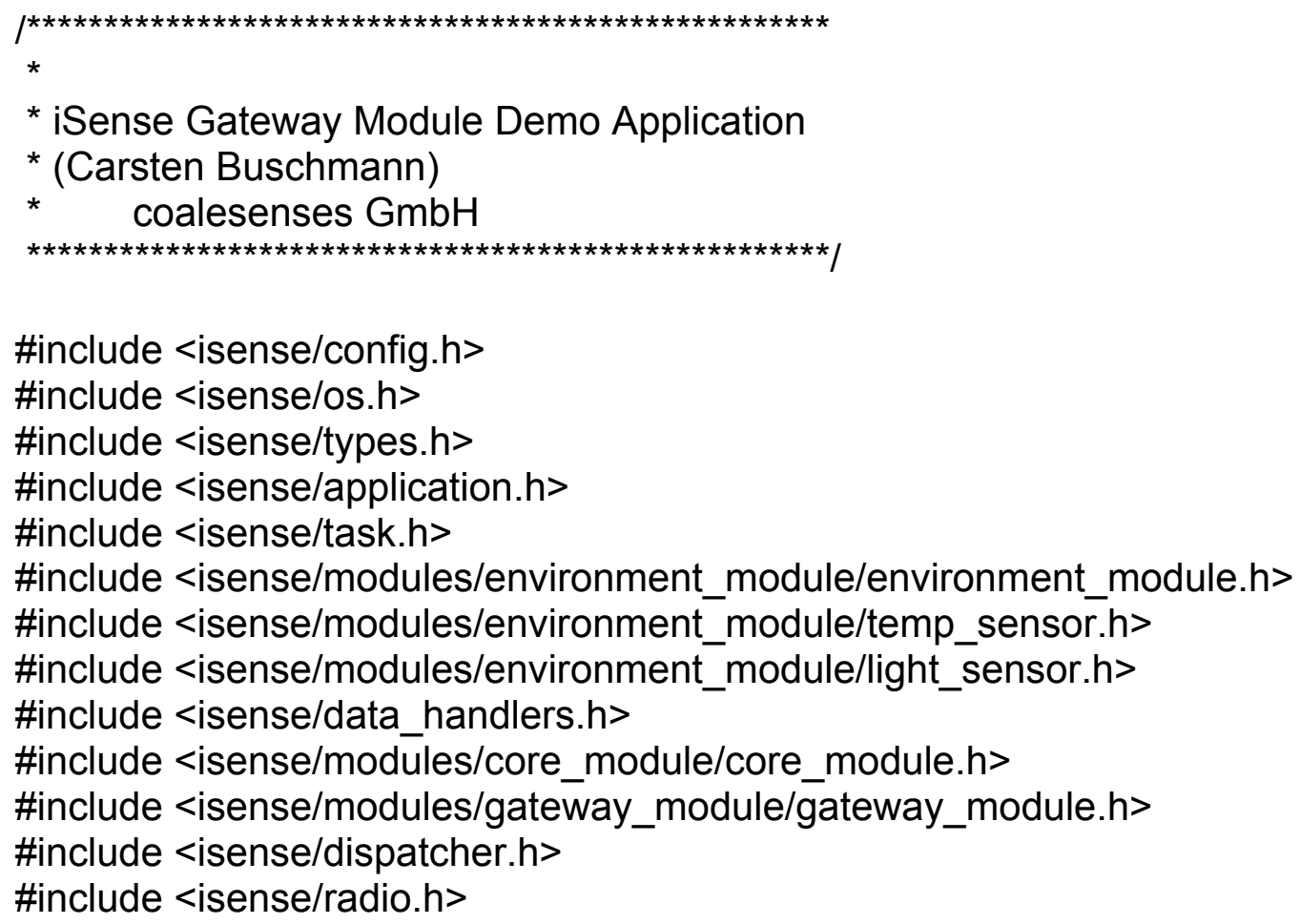

using namespace isense;

//-

class Envia :

public Application, public Task, public Int8DataHandler, public Uint32DataHandler, public isense::Sender, public isense::Receiver 
public:

// constructor

Envia(Os \&os);

// inherited from application, called upon device boot void boot();

// inherited from Task, called when

// a task is due

virtual void execute( void* $^{*}$ userdata );

// inherited from Int8DataHandler, called by the temperature

// sensor if the temperature threshold is exceeded

void handle_int8_data( int8 value );

// inherited from UInt32DataHandler, called by the light

// sensor upon luminance changes

void handle_uint32_data( uint32 value );

void send();

///From isense::Receiver

virtual void receive (uint8 len, const uint8 * buf, ISENSE_RADIO_ADDR_TYPE src_addr, ISENSE_RADIO_ADDR_TYPE dest_addr, uint16 signal_strength, uint16 signal_quality, uint8 sequence_no, uint8 interface, Time rx_time) ;

///From isense::Sender

virtual void confirm (uint8 state, uint8 tries, isense::Time time) ;

private:

// pointer to the temp/lum sensor

EnvironmentModule* em_;

//Pointer to CoreModule instance

CoreModule* cm_;

//Pointer to GatewayModule instance

GatewayModule* gwm_;

\}

//-

Envia::

Envia(Os \&_os) : 


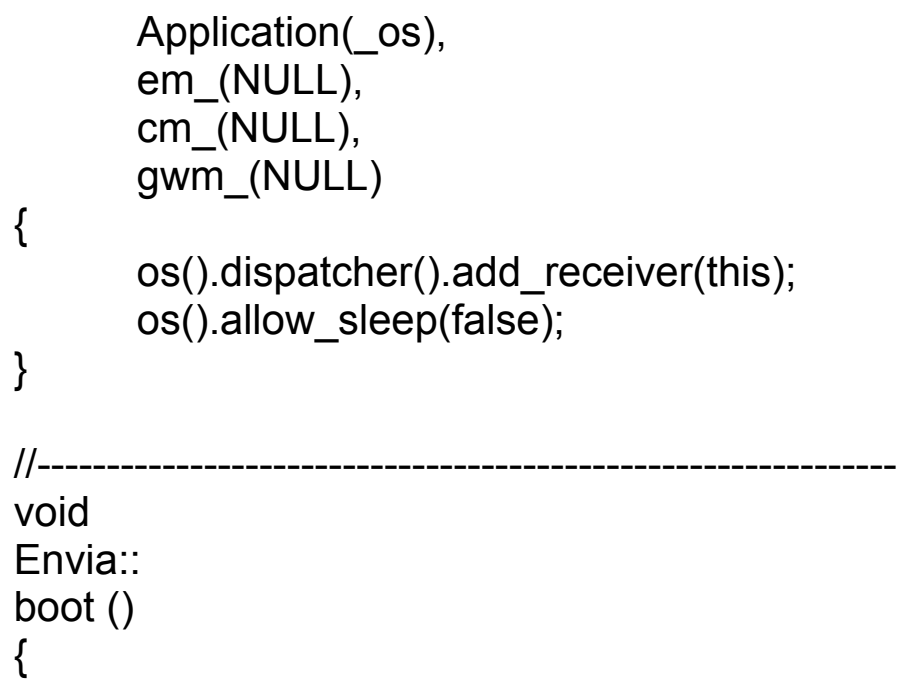

//create CoreModule instance

$\mathrm{cm}_{-}=$new CoreModule(os());

if (cm_! $=$ NULL) // new was successful

\{

//switch on Core Module LED to show device is awake

\} else cm_->led_on(); CoreModuleln")

os().fatal("ERROR: Could not allocate memory for

//create GatewayModule instance

gwm_ = new GatewayModule(os());

if (gwm_! = NULL) // new was successful

\{

//switch on Gateway Module LED to show module is working

\}else gwm_->led_on(0);

os().fatal("ERROR: Could not allocate memory for GatewayModuleln");

// output boot notification message

\#if (ISENSE_RADIO_ADDRESS_LENGTH == 16)

os().debug("Booting Environment Module Demo Application, $\mathrm{id}=\% \mathrm{x} \ln ", \mathrm{os}() \cdot \operatorname{id}())$;

\#else

$\mathrm{id}=\%|x| n "$, os ()$\cdot \mathrm{id}())$;

os().debug("Booting Environment Module Demo Application,

\#endif

// create EnvironmentModule instance 


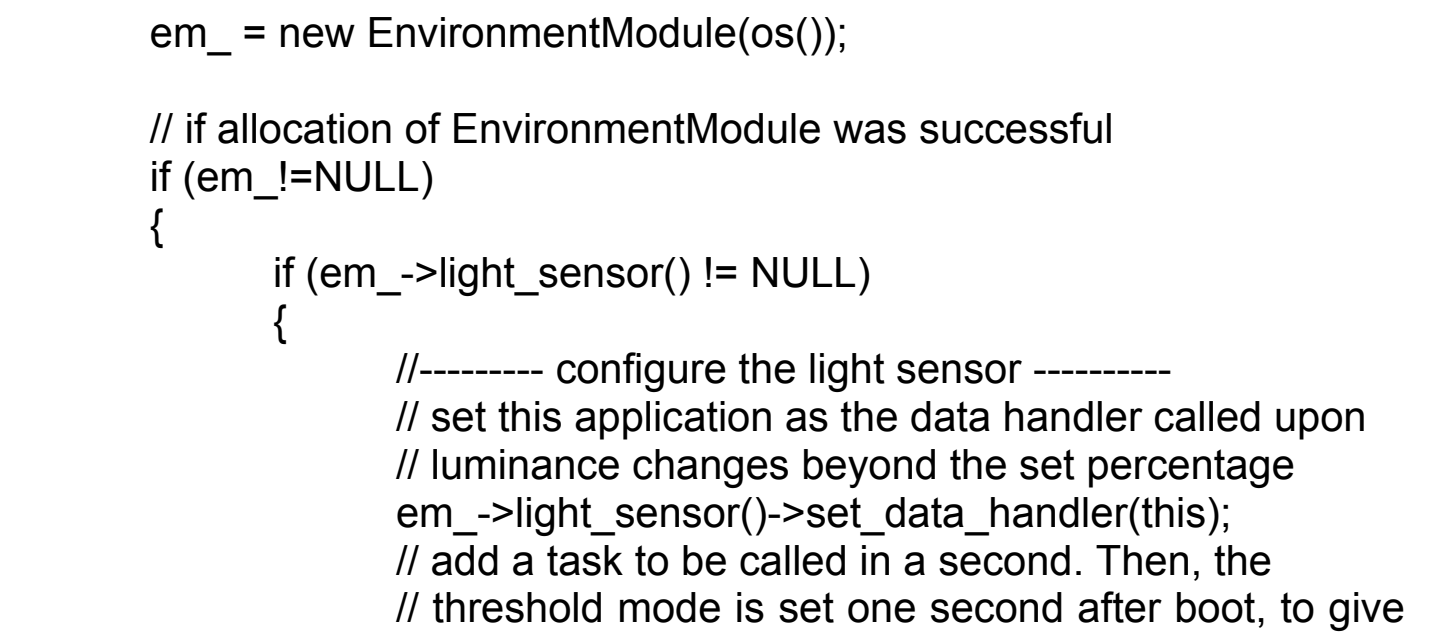

the

(void*)TASK_SET_LIGHT_THRES̄HOLD);

// sensor time for its first conversion

os().add task in(Time(2,0), this, \} else

os().fatal("ERROR: Could not allocate light sensorln");

if (em_->temp_sensor() != NULL)

\{

//--------- configure the light sensor ----------

// set the temperature threshold to $35>\mathrm{C}$ and the

hysteresis

sensorln");

// value to $30 \gg \mathrm{C}$

em_->temp_sensor()->set_threshold(35,30);

// set this application as the data handler called if

// temperature threshold is exceed or falls back below

// the hysteresis value

\} else

em_->temp_sensor()->set_data_handler(this);

os().fatal("ERROR: Could not allocate temp

// enable the Environmental Sensor Module,

// including its sensors

em_->enable(true);

readings

// register task to be called in a $\mathrm{x}$ time for periodic sensor (void*)TASK_READ_SENSORS);

\} else 


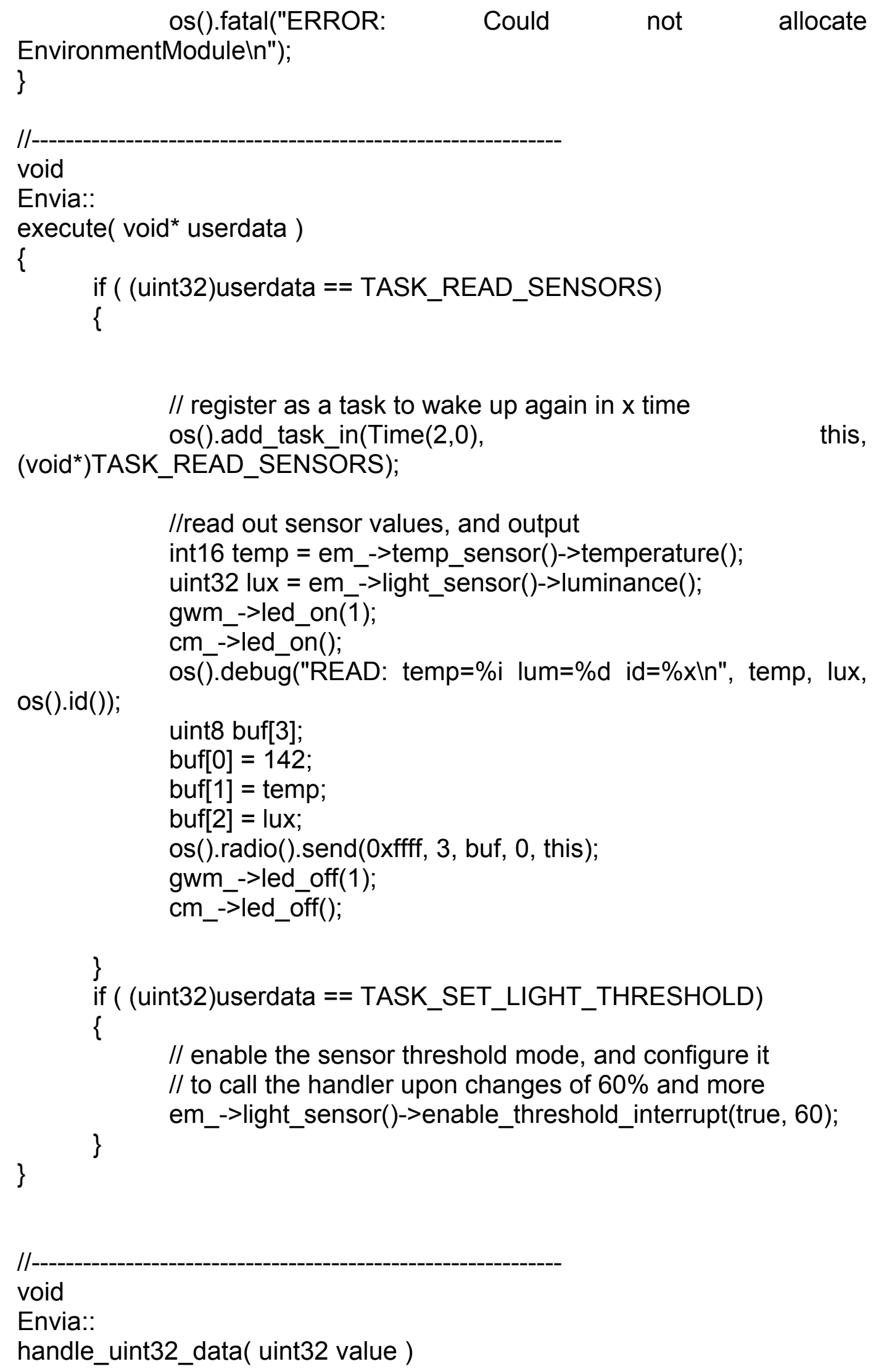


// lighting conditions changed --> output value

// envia (por radio) el paquete de tipo $144=$ threshold de lum

os().fatal("THRESHOLD: Threshold exceeded: lum=\%d id=\%xln", value, os().id());

uint8 buf[2];

buf[0] = 144;

buf[1] = value;

os().radio().send(0xffff, 2, buf, 0, this);

\}

/l

void

Envia::

handle_int8_data( int8 value )

\{

// temperature threshold exceeded --> output value

// envia (por radio) el paquete de tipo $143=$ threshold de temp

os().fatal("THRESHOLD: Limite sobrepasado: temp=\%i id=\%xln",

value, os().id());

uint8 buf[2];

buf[0] = 143;

buf[1] = value;

\}

os().radio().send(0xffff, 2, buf, 0 , this);

$/ /$

void Envia::

send()

\{

os().debug("Sending message to the broadcast address");

uint8 buf[2];

buf $[0]=142$;

buf $[1]=1$;

os().radio().send(0xffff, 2, buf, 0, this);

\}

/I

void Envia::

receive (uint8 len, const uint8 * buf, ISENSE_RADIO_ADDR_TYPE src_addr, ISENSE_RADIO_ADDR_TYPE dest_addr, uint16 signal_strength, uint16 signal_quality, uint8 sequence_no, uint8 interface, Time rx_time) 
\{

/los().debug("Receive message from \%x Packet Type \%i and rssi $\% d "$, src_addr, buf[0], signal_strength); src_addr);

//os().debug("READ: temp=\%i lum=\%i id=\%xln", buf[1], buf[2],

$1^{*}$

* buf[0] indica el tipo de dato que se recibe:

* $142=$ READ

* $\quad 143=$ THRESHOLD temp

* $\quad 144=$ THRESHOLD lum

$*$

if(buf[0] $==142)\{$

buf[2], src_addr);

os().debug("READ: temp=\%i lum=\%i id=\%xln", buf[1],

\}

if(buf[0] $==143)\{$

os().fatal("THRESHOLD: Threshold exceeded: temp=\%i $\mathrm{id}=\% x \ln "$, buf[1], src_addr);

\}

if(buf[0] $==144)\{$

os().fatal("THRESHOLD: Threshold exceeded: lum=\%i $\mathrm{id}=\% \mathrm{x} \backslash \mathrm{n} "$, buf[1], src_addr);

\}

\}

//-

void Envia::

confirm (uint8 state, uint8 tries, isense::Time time)

\{

//os().debug("Confirm sending message with state $=\% \mathrm{x}$ and tries $=\% \mathrm{i} "$, state, tries);

\}

//-

Application* application_factory(Os \&os)

\{

//create application instance

\} return new Envia(os); 


\section{Apéndice D: Script de Envío de datos a Twitter}

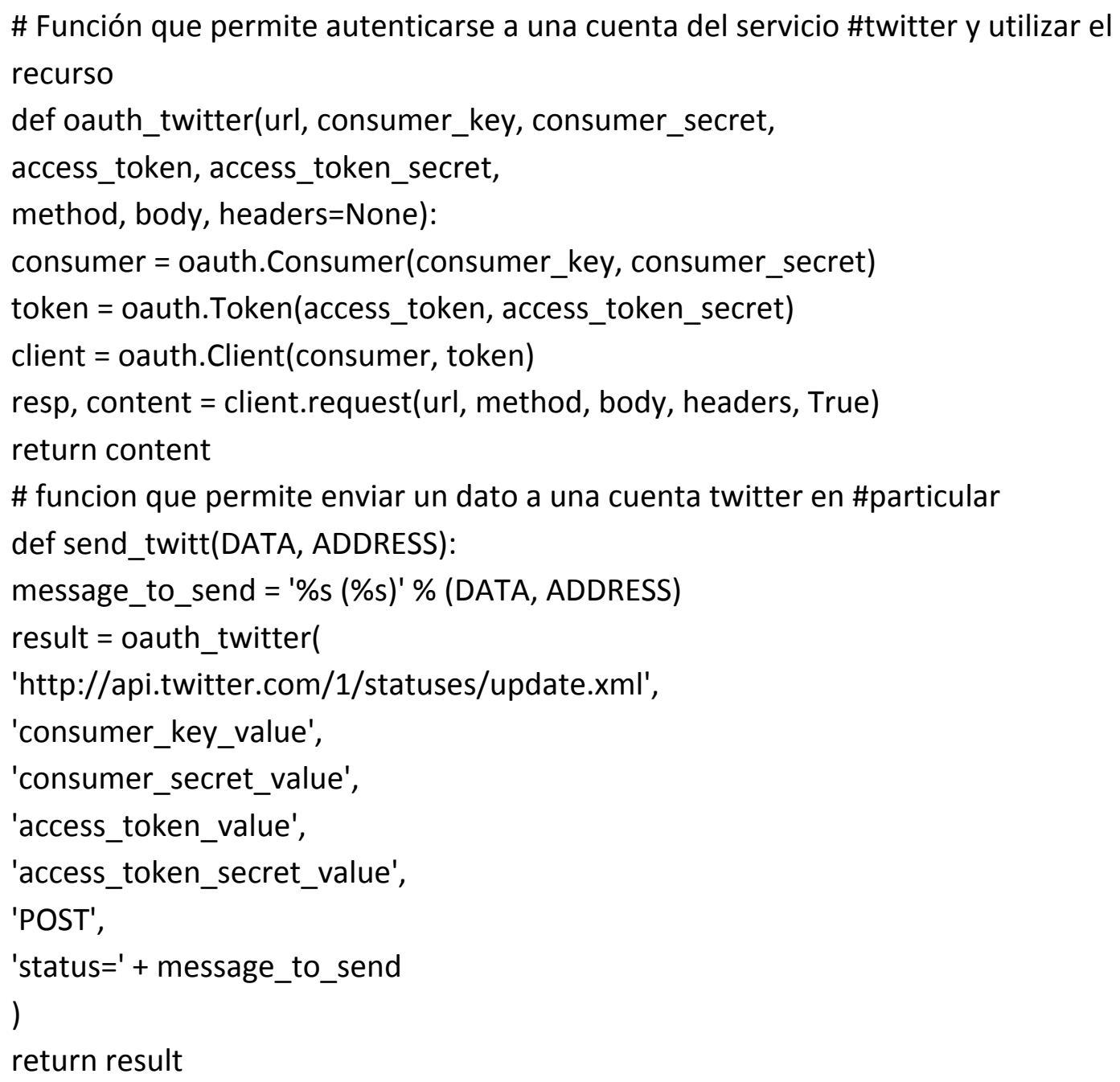


Apéndice E: Trabajos relacionados al proyecto 
Por la presente dejo constancia que el Proyecto de investigación "Hacia la programación de sensores inalámbricos en la forma web 2.0" bajo la dirección del Mgter.

Eduardo O. Sosa, de vigencia 01/01/2010 hasta el 31/12/2011, presentado en esta Secretaría y se encuentra en etapa de evaluación de los evaluadores externos, contándose con una evaluación positiva hasta el momento.

Se expide la presente constancia en la ciudad de Posadas a los 2 días del mes de Febrero de 2011.

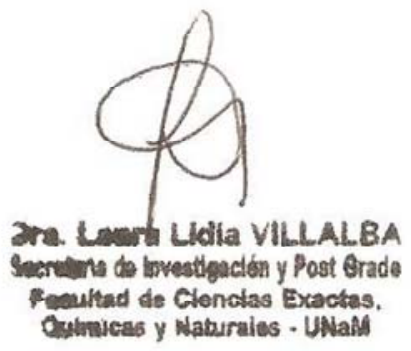

secip@fceqyn.unam.edu.ar

Facultad de Ciencias Exactas, Químicas y Naturales

Universidad Nacional de Misiones

Félix de Azara 1552 - 3300 Posadas, Misiones

Argentina 


\title{
Towards a Global Sensor Network Interconnection. A German- Argentinean Field Trial
}

\author{
E.O. Sosa ${ }^{1}$, S. Fischer ${ }^{2}$, F.J. Díaz ${ }^{3}$ \\ Facultad de Ciencias Exactas, Químicas y Naturales, Universidad Nacional de Misiones \\ es@fceqyn.unam.edu.ar \\ ${ }^{2}$ Institute für Telematik, Universität zu Lübeck \\ fischer@itm.uni-luebeck.de \\ ${ }^{3}$ Laboratorio de Nuevas Tecnologías Informáticas, Universidad Nacional de La Plata \\ jdiaz@info.unlp.edu.ar
}

\begin{abstract}
In this project universities from Germany and Argentina intend to set up a wireless sensor network in South America, specifically in the Yaboti Biosphere Reserve (YBR) in the Province of Misiones, Argentina. This project is granted by the Science and Technology Ministry in Argentina, Federal Ministry of Education in Germany, and the local Government in Misiones.

The new network pretends to be connected to the European network Wireless Sensors Testbeds, WISEBED[1] which has been established in June 2008, and it will deliver realworld data concerning the environment in these parks inside the YBR to everybody in the world who is interested. A second network might be established close to the sea coast of the Province of Buenos Aires near the Universidad Nacional de La Plata.

Besides the training and expertise provided, the project will be incorporated to the regular courses of the Magister on Data Networks as well as in the Doctorate on Informatics Sciences, and will also produce several post-graduate thesis.

WISEBED is part of the European Future Internet strategy, which is also broadly discussed on the German national level. An important aspect of Future Internet is the seamless inclusion of wireless sensor networks in order to make access to all kinds of realworld data possible for everybody over the Internet. As such, this project is a perfect extension to WISEBED.

All three partners will cooperate in order to set up this network and connect it to WISEBED: on the German side, it is the Institute of Telematics at the University of Lübeck, which also leads the WISEBED consortium. On the Argentinean side, it is the Universidad Nacional de La Plata, which has the capability of being counterpart to the European project, because of its academic level and the people involved. The Universidad Nacional de Misiones, a very young university, because of its location, is a necessary partner in order to provide the backup for the success of the endeavour.

It needs to be mentioned the commitment of the Government in Misiones, which monitors the provincial parks, and has agreed to provide the authorization for the setup of the network.

This work would provide significant results to the area from the theoretical and operative point of view. They would also promote many graduate and postgraduate courses and
\end{abstract}


activities alike not so common in the actual curricula of the universities involved in the project.

We are creating sensor network prototypes to both learn something about the practical implications of this technology and to enhance the field of ubiquitous computing in combination with the Future Internet. Apart from that, an important aspect of this project is the transfer of knowledge, namely traditional university courses, and by hands-on training in order to enable people to quickly set up successful sensor network prototypes.

\section{Introduction}

A standard scientific method for understanding complicated situations is to analyze them in a top-down, hierarchical manner. This approach also works well for organizing a large variety of structures; that is why a similar hierarchical, centralized approach has worked extremely well employing computers in so many aspects of our life. On the other hand, our world has become increasingly complex. The resulting challenges have become so demanding that it is impossible to ignore that a large variety of systems have a very different structure: the stability and effectiveness of our modern political, social and economic structures relies on the fact that they are based on decentralized, distributed and self-organizing mechanisms. The Internet is one of the success stories of such an approach in technology, and is has had a tremendous impact on many aspects of our lives. At this point, we are just beginning to see the effects of turning a vast number of heterogeneous objects into one large and decentralized network; in particular, the large-scale effects of the interaction between hardware, software, algorithms and data are just starting to show, and many of the resulting emerging phenomena come by surprises, rather than by design.

Turning from centralized algorithms for individual to large-scale mechanisms for decentralized and self-organizing networks has been one of the crucial paradigm shifts of computer science and its applications in recent years.

Until very recently, scientific efforts for studying computing methodologies for decentralized complex systems have been very limited. A particularly promising and hot research area has been the design and analysis of wireless sensor networks (WSN).

Hidden away in the extreme northeast of Argentina; is the Province of Misiones, sandwiched between Brazil and Paraguay. This Province is of considerable importance for conservation because it contains what is now the largest contiguous tract of southern Atlantic rainforest. Much of the remaining forest is in the area that has been gazetteered as the Yabotí Biosphere Reserve (YBR).

The Atlantic coastal rainforests of South America are most important because of their high level of endemism of plants and animals. They are one of the designated hotspots of priority for conservation. These are the areas considered both biologically richest and the most endangered of all terrestrial ecosystems. These forests are also one of the most diverse in the world. In spite of such a rich diversity and endemism, only about six percent of the Atlantic forest remains. The protection of the remainder is of vital importance [2].

Distributed, wireless, microsensor networks will enable myriad applications for sensing and controlling the physical world. A WSN is a network made of many devices using sensors. This sensors are also called nanocomputers. Usually these devices are small and inexpensive, so that they can be produced and deployed in large numbers[7].

The objective of a WSN is to monitor an environment at close range with high spatial and temporal resolutions. Examples of applications are monitoring and evaluating the health status of machines; environmental, medical, food-safety, and habitat; as well as energy management, inventory control, building automation, etc. Monitor the YBR is a very 
important goal. Now, it is quite clear the relation that there exists between WSN and its deployment at the YBR.

Sensor Node Hardware: while the first generation of wireless sensor networking hardware was energy-hungry and slow in computation and communication, the second or third generation available today has matured significantly. Reliable, standardized and high bandwidth radios of up to $250 \mathrm{kBits} / \mathrm{s}$ replaced old proprietary hardware, and fast but saving microcontrollers entered the stage. Both developments help to shorten the activity periods of the nodes and hence save power, while ultra low power sleep modes save power when not active. A quandary is whether to use a voltage regulator or not: while it reduces efficiency because of regulation wastes, it helps to completely empty the batteries.

Lately software controlled voltage regulators solved that problem. A number of WSN platforms offered by Crossbow are targeted at research and commercial use. Some of them are based on Atmel AtMega 128 controllers, and feature low bandwidth radios (MICA2 [8]) or high data rate IEEE 802.15.4 radios (MICAz [9], IRIS [10]). They can be combined with a number of sensor boards. The TelosB [11] combines a fast radio with a TI MSP430 controller and on board sensors. The imote2 [12] augments an IEEE 802.15.4 radio with a high performance XScale processor. All of these platforms do not feature a voltage regulator. The BTnodes [13] developed by the ETH Zrich are primarily targeted at researchers. They offer two radios, a Bluetooth and an ISM proprietary one, combined with an AtMega controller. The ScatterWeb GmbH offers two lines of WSN hardware. Both are based on a TI MSP430 radio and a proprietary rate ISM band radio. The MSB430 [14] is targeted at researchers, lacks a voltage regulator but includes onboard sensors. The ScatterNodes [15] are intended for commercial use, they feature a regulator but no sensors. The coalesenses $\mathrm{GmbH}$ offers the iSense platform [16], targeted at both research and commercial use. It is the first WSN hardware based on a single chip solution comprising an IEEE 802.15.4 radio and 32 bit controller. It features a software controllable voltage regulator and can be extended by a number of sensor and other interface boards.

Existing testbeds. So far, number and size of actual test beds for sensor networks have been rather limited. Almost all of these efforts have been struggling with a number of different issues:

- Hardware. Developing small-scale devices that are capable of carrying out specific sensing activities, as well as storing and processing data, and also can communicate via wireless radio.

- Interfaces, is a rather tedious and time-consuming task. Connecting them in order to build network structures needs new approaches due to resource constraints and the typical operation area (outdoors, remote).

- Software. Dealing with the limitations of small-scale special-purpose computing devices makes it very challenging to develop appropriate software, so far requiring custom-made software solutions.

- Algorithms. The classical theory and practice of algorithms focuses on a single central processing unit. Dealing with the challenges of designing algorithms for wellorganized, large-scale distributed systems requires new algorithmic methods.

- Data. The large volume of collected sensing information, as well as the communication overhead gives rise to huge amounts of data. Storing, organizing, and evaluating this data in a useful and practical manner pose completely new challenges.

It is particularly striking that these aspects are strongly interconnected: e.g., shrinking hardware imposes new constraints and challenges on the design of software, algorithms, and data maintenance; increasing data traffic does the same for hardware, software and algorithms, etc. 
There exists several testbeds around the world. The target of some of them was to build and demonstrate a large-scale outdoors sensor testbed to be used in a multi-target tracking application. Others were designed having in mind that testbed should be both open and easy to use for other researchers. Some of them are open only to authorized users. Among others we can mention Trio testbed [17], was one of the largest wireless sensor testbeds, indoors and outdoors, built yet. MoteLab [18] is an indoors sensor network testbed on the campus of Harvard University. TWIST testbed [19] resides indoors in a building in the campus of the Technical University of Berlin. TutorNet testbed [20] uses a 3-tier network topology with testbed servers, gateway stations, and sensor nodes, which feature USB connections to the gateway stations.

\section{Project description}

The genesis of this project resulted from a research visit of a teacher-researcher from the Facultad de Ciencias Exactas, Químicas y Naturales FCEQyN[5] of the Universidad Nacional de Misiones, at the University of Lübeck in the summer of 2007. At present, this teacher works on WSN in order to get his $\mathrm{PhD}$ degree at the Universidad Nacional de La Plata (UNLP).

During this 2-month-stay, the two partners developed the principle concept for such a project and then decided to look for additional partners and frameworks in which the project could be realized. In Argentina, the Laboratorio de Nuevas Tecnologías Informáticas LINTI from the UNLP [4] was asked to join in. In Germany, meanwhile, Institute of Telematics ITM [3] of the University of Lübeck set up the WISEBED consortium and applied for European funding which was granted in the beginning of 2008.

The three partners intend to set up a wireless sensor network in Argentina, specifically in the Reserve Biosphere Yabotí. The institutions participating in this project, will cooperate in order to set up this network and connect it to WISEBED. On the German side it is the ITM, on the Argentinean side, it is the LINTI that has the capability of being counterpart to the European project, because of its academic level and the people involved. The FCEQyN, because of its location; is a necessary partner in order to provide the backup for the success of the endeavour.

\section{Objectives and goals}

In particular, we plan to do the following.

- To deploy several wireless sensor devices in YBR to use for evaluating and testing solutions at large scale.

- To operate the testbed to collect traces of data from the physical environment and derive models of real-life situations and scenarios. These scenarios will be used to evaluate the performance of algorithms and systems and draw conclusions on their operation and how it can be improved.

- We will interconnect this wireless networks with the Internet and provide a virtual unifying laboratory to enable testing and benchmarking, in a controlled way, in different "real-life" situations.

- Allow researchers to use the facility remotely, thus reducing the need for a local, private testbed and, more importantly, reducing the cost for conducting all-rounded research.

- To share our results that could be directly used in future experiments as reference for benchmarking purposes 
- We expect that our involvement in the above activities will lead to the development of a collective understanding on how to conduct research on wireless sensor networks and the Internet, starting out from concrete applications.

\section{Methods}

We intend to make this network available to the scientific community and disseminate our methodology to research groups of both theoretical and practical background. It is our goal to establish a process of joint research activities, in which both theoreticians and practitioners will get together and use of the facilities in order to interact and put the methodology into action. A broad basis for a strongly interconnected, interdisciplinary research community would be provided to the German and Argentinean research groups and universities, leading to the use of the results of the project.

Our project will try to overcome some of these impediments by exploring the logical next step: building a network of networks. More specifically, we propose to build a network testbed, together with a unified and universal approach to software, algorithms, and data. Connecting sensory networks to the Internet creates endless opportunities for applications and services, new emerging models of operation. Users will be able to get real-time data from the physical world for everything, everywhere and anytime

Mark Weiser, the inventor of the term "Ubiquitous Computing" -wireless sensor networks are an enabling technology for ubiquitous computing-, was convinced that "the research method for ubiquitous computing is standard experimental computer science: the construction of working prototypes of the necessary infrastructure in sufficient quantity to debug the viability of the systems in everyday use, using ourselves and a few colleagues as guinea pigs". This is exactly the method we are following in this project: we are creating sensor network prototypes to both learn something about the practical implications of this technology and to advance the field of ubiquitous computing in combination with the Future Internet. An important aspect of this project is transfer of knowledge which we implement both by traditional courses in a university environment and by hands-on training. That will help to enable people to quickly set up successful sensor network prototypes.

\section{Expected results and its uses}

The WSN to be deployed at YBR will deliver real-world data concerning the environment in the parks inside the Yabotí to everybody in the world who is interested. This network would be the first one which will join the WISEBED project.

WISEBED is part of the European Future Internet strategy, which is also broadly discussed on the Germany national level [6]. The leading goal of WISEBED is the establishment of a distributed large-scale sensor network testbed which will be made accessible to the European research community. For that purpose, a variety of tasks has to be fulfilled and the WISEBED consortium has been carefully selected in order to be able to optimally fulfill these tasks. The main strength of this proposal is that it brings together a group of scientists who provide the right mix for new concepts that allow dealing with distributed algorithm engineering and large-scale experimentation. Also, the project composition demonstrates an appropriately designed mix of partners from academia and research institutes. An important aspect of Future Internet is the seamless inclusion of wireless sensor networks in order to make access to all kinds of real-world data possible for everybody over the Internet. As such, this project at YBR is a perfect extension to the WISEBED one. A second network might be established close to the Ocean coast of the Province of Buenos Aires near the UNLP. 
Besides the training and expertise provided, the project will be incorporated in the regular courses of the Magister on Data Networks in the UNLP and also will produce several postgraduate thesis. This work would provide significant result to the area from the theoretical and operative point of view. They would also promote many graduate and postgraduate courses and activities alike not so common in the actual curricula of all universities that are taking part in the project.

This project will provide a WSN testbed as starting point to bring together different similar facilities across Argentina and create a federation of distributed test laboratories. This will provide services for allowing advances in theoretical computer systems to be tested, at least as a proof-of-concept. The feasibility of the new concepts and verify their large-scale effects could be assessed. We will engage on implementing recent theoretical results on algorithms, mechanisms and protocols and transform them into software that is independent from current technologies. Furthermore, we will evaluate code independence by using the different hardware available across WISEBED and place the resulting code under the scrutiny of largescale simulations and experiments. This evaluation will provide valuable feedback and derive further requirements, orientations and inputs for the long-term research.

Wireless sensor networks are considered to be one of the key technologies in information technology in the coming years. It is the goal of the project WISEBED - and thus also of this associated project - to clear the path for a broad usage of such networks. Projects like this one intend to show the possibilities that wireless sensor networks offer to a broad range of industries. By show-casing the project results, we expect to create major interest in several branches of both European and Argentinean economy.

\section{Benefits for each country}

By cooperating with a non-European partner, Germany/Europe will be able to extend its Future Internet strategy to other parts of the world and thus challenge the leadership of other countries in this area. Additionally, German technology (the sensor nodes and the sensor network algorithms) will be exported to external countries; as a result, Germany will improve its position with respect to competitive advantages in this field. Finally, European researchers will profit a lot from the extension of WISEBED in terms of size, but also because they get access to real-world data - which is not so common in today's sensor net projects.

Argentina will profit because they get access to European high-tech information technology and the knowledge developed in Europe. They will learn from German researchers about the technologies in the rapidly emerging field of wireless sensor networks, and they will get to use real sensor network technology. Also, Argentina will be able to test new technology for monitoring and protecting their national resources, especially in oxygen reserves of the world, like the YBR.

\section{Utilization of results}

Wireless sensor networks are considered to be one of the key technologies in information technology in the coming years. It is the goal of the project WISEBED - and thus also of this associated project - to clear the path for a broad usage of such networks. Projects like this one intend to show the possibilities that wireless sensor networks offer to a broad range of industries. By show-casing the project results, we expect to create major interest in several branches of both European and Argentinean economy. 


\section{Conclusions and Future Work}

We strongly believe that in the future, researchers will be able to bypass most of these time consuming issues and even totally avoid the need of developing a local experimental testbed. They will have access to experimental facilities freely available for use that offer a wide range of technologies for which applications can be tested in real-life scenarios. They will have the support of a group of experts that can offer help on how to develop algorithms, overcome technicalities due to the hardware limitations and interpret results acquired from the evaluation of the application.

Due to the close cooperation with the WISEBED project, at least two PhD students will be in close contact with this project and provide input whenever suitable. In addition, master thesis will be executed in the context of this project. On the Argentinean side, this facility would provide significant results to the area, from the theoretical and operative point of view.

\section{Acknowledgments}

We thank the Ministerio de Ciencia, Tecnología e Innovación Productiva in Argentina, the Bundesministerium für Bildung und Forschung in Germany, and the local Government in Misiones for supporting this project. We also thank Dennis Pfisterer from ITM of the University of Lübeck and Luis Marrone from LINTI at Universidad Nacional de La Plata for their contributions.

\section{References}

[1] Wireless Sensor Network Testbeds, Retrieved Sept 22, 2009, from http://www.wisebed.eu/

[2]International Society of Environmental Botanists, "The Protection of the Yabotí Biosphere Reserve, Misiones, Argentina and its Guaraní People”, Vol. 13 Nr. 2 (2007).

[3] Laboratorio de Investigación de Nuevas tecnologías Informáticas, Universidad Nacional de La Plata, http://www.linti.unlp.edu.ar .

[4] ITM, Institut für Telematik, Universität zu Lübeck, http://www.itm.uni-luebeck.de

[5] FCEQyN, Facultad de Ciencias Exactas, Químicas y Naturales, Universidad Nacional de Misiones, http://www.fceqyn.unam.edu.ar/ .

[6]Future Internet Aufruf zur Einreichung von Beiträgen http://www.future-internet.org/

[7] XiangYang Li, "Wireless Ad Hoc and Sensor Networks", Cambridge University Press (2008)

[8] Crossbow MICA2 868, 916 MHz. http://www.xbow.com/Products/productdetails . aspx?sid=174.

[9] Crossbow MICAz 2.4GHz. http://www.xbow.com/Products/productdetails.aspx ?sid=164.

[10] Crossbow IRIS 2.4GHz. http://www.xbow.com/Products/productdetails.aspx? sid=264.

[11] Crossbow TelosB. http://www.xbow.com/Products/productdetails.aspx?sid=252.

[12] Crossbow imote2. http://www.xbow.com/Products/productdetails.aspx?sid=253.

[13] BTnodes - A Distributed Environment for Prototyping Ad Hoc Networks. 
http://www.btnode.ethz.ch/.

[14] ScatterWeb. http://www.scatterweb.de/content/products/research line/.

[15] ScatterWeb ScatterNode. http://www.scatterweb.de/content/products/industry line/scatternode.de.html.

[16] Coalesenses iSense - A modular hardware and software platform for wireless sensor networks. http://www.coalesenses.com/isense.

[17] P. Dutta et al. Trio: enabling sustainable and scalable outdoor wireless sensor network deployments. In 5th International conference on Information processing in sensor networks (IPSN), pages 407-415. ACM Press, New York, 2006.

[18] G. Werner-Allen, P. Swieskowski, and M. Welsh. Motelab: A wireless sensor network testbed.In Fourth International Conference on Information Processing in Sensor Networks (IPSN), 2005. Special Track on Platform Tools and Design Methods for Network Embedded Sensors (SPOTS), IEEE, Piscataway, NJ.

[19] V. Handziski, A. Kopke, A.Willig, and A.Wolisz. Twist: A scalable and reconfigurable wireless sensor network testbed for indoor deployments. Technical Report TKN-05-008, November 2005.

[20] Tutornet: A tiered wireless sensor network testbed. http://enl.usc.edu/projects/ tutornet/.

[21] B .N. Chun et al. Mirage: A microeconomic resource allocation system for sensornet testbeds. In 2nd IEEE Workshop on Embedded Networked Sensors, 2005. 


\section{Hacia una Red Global de Sensores Interconectados. Un Ensayo Experimental Argentino-Alemán}

Sosa, Eduardo O./ Díaz, Francisco J./ Marrone, Luis A.

Facultad de Ciencias Exactas, Químicas y Naturales - Universidad Nacional de Misiones

Laboratorio de Investigación de Nuevas Tecnologías - Universidad Nacional de La Plata

eososa@unam.edu.ar; jdiaz,lmarrone@info.unlp.edu.ar

\section{Resumen}

Las redes de sensores inalámbricos son una de las tecnologías fundamentales en la Internet del futuro, y representan la expansión de Internet al mundo real, permitiendo acceder a indicadores que pueden ser consultados desde cualquier punto del orbe en tiempo real. En junio de 2008 instituciones académicas y de investigación europeas, en un esfuerzo conjunto, ponen en marcha el proyecto WISEBED, como parte de la "Estrategia Europea para Internet del Futuro" respaldada fuertemente por la Sociedad Alemana de Informática. El objetivo del WISEBED es proveer una infraestructura de interconexion de redes de sensores inalámbricos a gran escala con fines de investigación que persigue un enfoque interdisciplinario.

En este contexto las Universidades de La Plata y Misiones de la República Argentina y la Universidad de Lübeck en la República Federal de Alemania generamos un proyecto conjunto, cuyo objetivo es instalar una red de sensores en la reserva biosfera Yaboty, en la provincia de Misiones. Esta red se interconectará con WISEBED en Europa, y permitirá acceder a los datos recogidos en ese ambiente natural desde cualquier punto de la red de redes. Es de notar que la red instalada en Yaboty será la primera red externa a los países de la Comunidad Europea, la cual pasaría a ser invitada del consorcio más allá del viejo continente.

Para Argentina es destacable la oportunidad de desarrollo, apropiación y aplicación de estas novísimas tecnologías en el campo de las TIC's, las que se complementan con nuevas medidas de protección y contralor de los ambientes naturales, que convivirán con las actuales hasta su superación operativa.

El resultado se aplicará al control de simulaciones y experimentos, a partir de los cuales se espera obtener información valiosa $\mathrm{y}$, fundamentalmente, nuevos requisitos, orientaciones e insumos para la investigación a largo plazo. Se pretende que los datos de estos laboratorios distribuidos estén a disposición de la comunidad científica argentina y europea, a fin de que otros grupos de investigación se beneficien con los mismos. En general, esto significa impulsar el nuevo paradigma de la informática distribuida y la autoorganización de estructuras a un nivel diferente.

La tareas coordinadas implican: a) adoptar la arquitectura del hardware y el software de los nodos. b) Transmitir experiencias necesarias para la operación de esta tecnología en el ámbito de la selva misionera. c) Desarrollar los programas aplicados en los nodos sensores inalámbricos.

Palabras clave: sensores inalámbricos, redes ad hoc, monitoreo ambiental. 


\section{Introducción}

Las redes de sensores inalámbricos son una de las tecnologías fundamentales en la Internet del futuro, y representa la expansión de Internet al mundo real, permitiendo acceder a indicadores que pueden ser consultados desde cualquier punto del orbe en tiempo real. Estos sensores son fuentes de información tan variados como lo son las medidas que realizan, los hay de temperatura, de luminosidad, de presión, de humedad, de velocidad, de aceleración, de presencia, de volumen y un sinfín de magnitudes que se nos ocurran. Si a estos sensores que nos reportan información valiosa para nuestras actividades, les añadimos la capacidad de comunicación inalámbrica y la posibilidad de formación de redes ad hoc, estamos en presencia de una red de Sensores Inalámbricos.

En junio de 2008 instituciones académicas y de investigación europeas, en un esfuerzo conjunto y financiado por la Comisión Europea, han puesto en marcha el proyecto WISEBED ${ }^{1}$ que forma parte de la "Estrategia Europea para Internet del Futuro" (FUTURO [2]), la cual está fuertemente respaldada por la Sociedad Alemana de Informática.

El objetivo del WISEBED es proveer una infraestructura de interconexión de redes de sensores inalámbricos a gran escala con fines de investigación, desde un enfoque interdisciplinario. La finalización del proyecto está fijada para mayo de 2011.

Como coordinador del consorcio WISEBED se ha designado a la Universidad de Lübeck, por su posicionamiento a la vanguardia de este tipo de investigaciones y aplicaciones tecnológicas. El financiamiento del proyecto por parte de Comisión Europea se realiza por intermedio del programa Tecnologías de la Información y Comunicación (WISEBED [1]); y las instituciones, a saber: las Universidades de Lübeck, Berlín y Braunschweig de Alemania, el Research Academic Computer Technology Institute de Grecia, la Universidad Politécnica de Cataluña de España, las Universidades de Berna y Ginebra de Suiza, Universidad de Delft de Holanda y la Universidad de Lancaster en el Reino Unido de Gran Bretaña.

En este contexto la $\mathrm{UNLP}^{2}$ y la $\mathrm{UNaM}^{3}$; de la República Argentina, y la Universidad de Lübeck; en la República Federal de Alemania, han generado un proyecto conjunto cuyo objetivo es instalar una red de sensores en la reserva biósfera Yabotí, en la provincia de Misiones. Oportunamente, esta red se interconectará con WISEBED en Europa, y permitirá acceder a los datos recogidos en ese ambiente natural desde cualquier punto de la red de redes. Es de notar que la red instalada en Yabotí será la primera red externa a los países de la Comunidad Europea, la cual pasaría a ser invitada del consorcio más allá del viejo continente.

\section{Contactos Previos, Impulsos}

A inicios de 2002, un profesor e investigador de la Universidad Nacional de Misiones fue invitado por el Instituto de Telemática ${ }^{4}$, de la Universidad de Karlsruhe en la República

\footnotetext{
${ }^{1}$ WISEBED: Wireless Sensors Testbed.

${ }^{2}$ Universidad Nacional de La Plata.

${ }^{3}$ Universidad Nacional de Misiones.

${ }^{4}$ Institut für Telematik, Universität Karlsruhe, http://www.tm.uka.de/
} 
Federal de Alemania, en el marco del programa de Reinvitaciones para ex becarios del $\mathrm{DAAD}^{5}$. Dicha invitación se realizó con el fin de participar en el proyecto denominado "IP on air" ${ }^{6}$. El docente e investigador de la UNaM, una vez finalizada la visita a Alemania y dentro del posgrado de la UNLP "Maestría en Redes de Datos"7 presentó a evaluación su tesis como Magíster en Redes de Datos ${ }^{8}$, el que fuera dirigido por profesores de la Facultad de Informática de la UNLP, y de la cual egresara en 2002.

En 2007 el mismo profesional fue invitado por el director del ITM $^{9}$-Institut für Telematik- de la Universidad de Lübeck, para participar en el proyecto "Sistemas Modernos de Comunicación".

En la formulación del proyecto de invitación al docente argentino establecía claramente que "...la educación y práctica juegan un rol fundamental en la llamada Sociedad de la Información y que la rápida y constante evolución de la ciencia y tecnología requiere una actualización y adecuación profesional, dado que la enseñanza en nuestros días, hasta ahora vista como actividad de aulas durante el año, debe ser vista en el contexto de un proceso de aprendizaje continuo. Aparte de ello, la innovación tecnológica ha contribuido al desarrollo de herramientas de soporte para la educación, y con ellas debe permitir planes flexibles e individuales de aprendizaje, proveyendo a la gente iguales oportunidades de acceso y de éxito en las actividades académicas y laborales."

Asimismo se ponía especial énfasis a los siguientes conceptos, “...La penetración de las $\mathrm{TIC}^{\prime} \mathrm{s}^{10}$, tanto en el campo de la educación como la capacitación laboral, requiere ineludiblemente que los estándares a ser adoptados y que los roles y métodos de enseñanza deban ser rediseñados para un mejor aprovechamiento de las oportunidades ofrecidas por la innovación tecnológica."

La elaboración conjunta de los objetivos de la visita a la universidad europea, se basaban en considerandos como “...los métodos de enseñanza deben ser diseñados con un modelo donde el usuario ocupe el centro de la escena de manera que sea pedagógicamente efectiva, y así evitar el impacto negativo de los educandos".

El resultado de esta visita a la Universidad del norte alemán se plasmó en dos acciones concretas: a) la firma de una carta de intención entre la Universidad Nacional de Misiones y el Instituto de Telemática; el que fuera refrendado por los rectores de ambas universidades; b) coordinación de realización de un trabajo de tesis de doctorado en la Universidad Nacional de La Plata sobre el tema "Redes Inalámbricas Ad Hoc y de sensores" dirigida por los directores de Institutos de Investigación de la Universidad de Lübeck y la UNLP, donde el doctorando es un docente de la UNaM.

\footnotetext{
${ }^{5}$ Deustscher Akademischer Austausch Dienst, Servicio Alemán de Intercambio Académico.

${ }^{6} \mathrm{http}: / /$ www.tm.uka.de/itm/WebMan/WebMan/view.php?view=projekte_detail\&id=9

${ }^{7} \mathrm{http} / / /$ postgrado.info.unlp.edu.ar/

8 “ANÁLISIS DE PROXIES COOPERATIVOS EN SISTEMAS EDUCATIVOS". (Observaciones y Mediciones realizadas en la Universidad de Karlsruhe, República Federal de Alemania). http://postgrado.info.unlp.edu.ar/Carrera/Magister/Redes\%20de\%20Datos/Tesis/PROXIESCOOPERATIVOS-EDUCATIVOS.pdf

${ }^{9} \mathrm{http}: / /$ www.itm.uni-luebeck.de/

${ }^{10}$ Tecnologías de la Información y Comunicación.
} 
Esta voluntad de realizaciones conjuntas entre las tres entidades se coronó con la presentación de un proyecto, en los ministerios de la República Federal de Alemania y de la República Argentina, solicitando se financien las visitas a los institutos y facultades en los dos países.

Teniendo en cuenta la simultaneidad de las actividades desarrolladas por el ITM, asumiendo la coordinación del proyecto WISEBED, y que el director del ITM es a su vez director de la tesis de doctorado mencionada anteriormente, y que se está llevando a cabo en la UNLP; estamos en presencia de una oportunidad estratégica, incomparable e importantísima para las instituciones participantes de nuestro país en el presente proyecto. Ésta se identifica como la posibilidad que el proyecto de sensores inalámbricos en la Reserva Biósfera Yabotí, sea considerada por el consorcio WISEBED como los primeros participantes no europeos en dicho proyecto durante el año 2009.

\section{Objetivos, Conceptos y Metas}

3.1. Objetivo: El objetivo de este proyecto es establecer una red de sensores inalámbricos en una reserva natural de la Provincia de Misiones, la que interconectada a un grupo de trabajo conjunto interuniversitario internacional, proveerá un nivel de infrastructura a gran escala en cuanto a redes de sensores inalámbricos para fines de investigación, persiguiendo un enfoque interdisciplinario que integra los aspectos de hardware, software, algoritmos y datos. Se pretende demostrar qué dispositivos de prueba en pequeña escala pueden reunirse para formar estructuras bien organizadas a gran escala, con la capacidad de hacer frente a escenarios dinámicos, tanto en la composición y ubicación.

\subsection{Relevancia del Tema}

El método científico estándar para llegar al entendimiento de situaciones complejas, consiste en analizarlas de una manera jerárquica, de arriba hacia abajo. Este método también es válido para la organización de una nueva cantidad de estructuras; es por ello que un análisis jerárquico similar y centralizado ha permitido el empleo de computadoras en muchos aspectos de nuestra vida diaria.

Por otro lado, nuestro mundo se ha vuelto cada vez más complejo. El resultado del análisis establece que es imposible ignorar que una gran variedad de sistemas poseen estructuras muy diferentes: la estabilidad de nuestra moderna estructura social, económica y política se basa en el hecho que están basados fundamentalmente en mecanismos descentralizados, distribuidos y autoorganizados. Internet es una de estas historias exitosas donde una visión tecnológica ha tenido un impacto tremendo en muchísimos aspectos de nuestras vidas.

En este momento estamos visualizando los efectos de vasto número de objetos heterogéneos en una red descentralizada; particularmente los efectos de la escala en la interacción entre hardware, software, algoritmos y datos, donde la mayoría de los fenómenos ocurren como forma de sorpresa y no como causa del diseño.

La migración de algoritmos centralizados para procesadores individuales a mecanismos de gran escala de redes descentralizadas y autoorganizadas, es el cambio primordial experimentado en el paradigma de las ciencias informáticas y sus aplicaciones en los años recientes. 
Hasta hace muy poco tiempo, los esfuerzos científicos eran muy exiguos para estudiar las metodologías de cómputo de sistemas descentralizados. Un área nueva y promisoria ha sido el análisis y diseño de las redes de sensores inalámbricos (WSN). Una WSN es la observación de un ambiente desde corta distancia y alta resolución espacial y temporal. Ejemplos de la aplicación de estos dispositivos es el control de equipos y máquinas; monitoreo ambiental, médico, seguridad alimentaria, hábitat, energía, control de inventario, automatización de edificios, etc.

\subsection{Descripción del Proyecto}

En este proyecto participan tres instituciones que cooperarán entre sí para instalar una red de sensores inalámbricos e interconectarla a WISEBED, proyecto que se encuentra operativo desde Junio del corriente año en la comunidad europea.

Como lugar de instalación de esta infraestructura de sensores inalámbricos se han establecido dos ambientes naturales que se adaptarían a la obtención de resultados prácticos válidos, no sólo a nivel académico y científico, sino también de parámetros definidos con anterioridad, que son de interés a las agencias ambientales provincial y nacional y que se involucrarán en el proyecto.

Las locaciones para la potencial instalación de los sensores inalámbricos son: a) La Reserva Biósfera Yabotí en la Provincia de Misiones y b) Reservas Naturales en la Provincia de Buenos Aires en el área geográfica abarcada por la UNLP en cercanía del Océano Atlántico.

Alemania está representada en el proyecto por el Instituto de Telemática de la Universidad de Lübeck (ITM [3]), institución que ha destinado recursos humanos de su propio presupuesto (dos investigadores semidedicación durante 2 años) apostando al éxito de la empresa conjunta. La contraparte argentina es compartida por el LINTI -Laboratorio de Investigación de Nuevas Tecnologías Informáticas (LINTI [4]) - de la Universidad Nacional de La Plata que involucró a dos Investigadores (profesores titulares) y la Facultad de Ciencias Exactas de la Universidad Nacional de Misiones (UNaM [5]); quien hiciera de nexo entre todas las partes involucradas, aporta un profesor adjunto con el $50 \%$ de su semidedicación. Este docente de la UNaM es a la vez doctorando en Ciencias Informáticas de UNLP ${ }^{11}$.

La UNaM, en esta primera parte del proyecto, es la participante necesaria e indispensable para asegurar el éxito, teniendo en cuenta su ubicación geográfica en la Provincia de Misiones; asegurando con esto el soporte local para el emprendimiento en lo atinente a logística y mantenimiento.

Asimismo el Gobierno de la Provincia de Misiones, y la herramienta institucional que lo representa para este tipo de proyectos: el Parque Tecnológico Misiones ${ }^{12}$ han demostrado su interés y aprobación a la instalación y desarrollo de este proyecto en el ámbito provincial, el cual proveerá en primer lugar al Ministerio de Ecología, Recursos Naturales y Turismo ${ }^{13}$, de una herramienta de punta para apuntalar en cada una de las tareas específicas desarrolladas por esa Secretaría de estado.

\subsection{Metas}

\footnotetext{
${ }^{11}$ http://postgrado.info.unlp.edu.ar/Carrera/Doctorado/Doctorado\%20en\%20Ciencias\%20Informaticas.html

12 Parque Tecnológico Misiones, http://www.ptmi.org.ar

${ }^{13} \mathrm{http}$ ://www.misiones.gov.ar/ecologia/_private/eco1.htm
} 
Cuando tratamos con sensores inalámbricos, debemos tener en cuenta que la disminución del tamaño del hardware impone limitaciones y desafíos en cuanto al diseño de software, algoritmos y de mantenimiento de datos; el incremento de tráfico afecta por igual a todos estos elementos. En este proyecto trataremos de superar estos impedimentos explorando la próxima etapa, y es tratando de establecer una red de redes.

Conectar los sensores a Internet crea una oportunidad infinita en cuanto a las aplicaciones y servicios sobre los nuevos modelos emergentes de operación. Los usuarios de Internet podrán ser capaces de obtener información en tiempo real del mundo físico sobre cualquier elemento, en cualquier lugar y en cualquier momento. Este tipo de investigaciones, hasta el día de hoy, han sido llevadas a cabo generalmente en paralelo, y muchas veces en forma aislada, lo que ha imposibilitado establecer un marco global unificado.

La meta principal del proyecto es obtener resultados valederos tanto desde el punto de vista práctico como desde lo teórico y académico; propendiendo con ello a promover actividades a nivel académico y científico de graduados y posgraduados, las que en la actualidad no son tan comunes en la currícula de las universidades argentinas.

El carácter multidisciplinario de las actividades involucradas en el proyecto aportarán indefectiblemente al estudio y modelización de tráfico en las redes ad-hoc, y aquello que se ha convertido en un tema de investigación sobre este tipo de redes y que se ha vuelto un tema de candente actualidad, el encaminamiento.

Las metas fundamentales de este proyecto se pueden establecer con los siguientes enunciados:

- Propender a facilitar el acceso a la infraestructura de sensores inalámbricos vía Internet de otros grupos de investigadores de cualquier parte del planeta, apuntando a la asociatividad con los de nuestro país.

- Producir material de entrenamiento de tal manera de facilitar los talleres y cursos de capacitación de investigadores y docentes en el tema de los sensores inalámbricos, orientando a estos al uso de la instalación experimental.

\section{Metodología}

Mark Weiser, el creador del término "Ubiquitous Computing" ${ }^{14}$, ha defendido el concepto de que el método de investigación para esta rama de la telemática es aplicar el método científico estándar de experimentación computacional, es decir: Construir los modelos prototipos de una infraestructura en suficiente cantidad para ir corrigiendo y modificando la viabilidad del sistema. Éste es el método que pretendemos utilizar en este proyecto, creando una red prototipo de sensores tanto para aprender sobre las implicaciones prácticas de la tecnología aplicada, como también avanzar en este campo de las TICs presentado en este caso como la Internet del futuro.

Se pretende instalar una infraestructura de sensores inalámbricos en la provincia de Misiones, donde se puedan llevar a cabo diferentes ensayos (testbesds) desde lugares remotos facilitando las actividades de investigación y desarrollo tecnológico. A partir de esto se podrán proveer servicios adaptados a proyectos de gran escala, al menos como

\footnotetext{
${ }^{14}$ Sistemas para lo cual no es necesaria la presencia física en la consola, pero reside ineludiblemente en el ambiente en el cual estamos insertos.
} 
"concepto de prueba", de manera de evaluar la adaptabilidad de las nuevas concepciones verificando sus efectos a otra escala. Estas actividades se concatenarán con resultados teóricos recientes sobre algoritmos, mecanismos y protocolos; transformándolos en software independiente de la tecnología actual. Posteriormente, podremos evaluar la independencia del código usando diferente hardware que estará disponible en WISEBED, validando los resultados a lo largo de simulaciones y experimentos a gran escala.

Esta evaluación proveerá la realimentación de requerimientos, orientaciones y nuevas líneas de trabajo en la investigación a largo plazo. Particularmente proponemos realizar lo siguiente:

- Instalar un número determinado de equipos sensors inalámbricos en una zona determinada de la Reserva Biósfera Yabotí, a fin de utilizarlos como evaluación y soluciones piloto a otro tipo de escala.

- Operar la infraestructura de los sensores a instalar recolectando datos del mundo real de manera de poder compararlos con resultados obtenidos en escenarios físicos ideales (WISEBED), a partir de los cuales se podrán evaluar las prestaciones de los algoritmos y sistemas para obtener conclusiones de su operación y como mejorar las prestaciones.

- Interconectar esta red de sensores inalámbricos a Internet proveyendo un laboratorio virtual unificado permitiendo ensayos y comparaciones, en forma controlada, en diferentes situaciones de la "vida real".

- Permitir a los investigadores utilizar la instalación remotamente reduciendo las necesidades de instalaciones locales y más importante aún, reducir drásticamente los costos de los trabajos de investigación en el tema.

A cada una de las tareas propuestas anteriormente se contrapone la existencia de ciertos riesgos significativos para el proyecto. Por esa razón se elaborará en consecuencia un plan de contingencia de manera tal de minimizarlos y eventualmente evitarlos por completo. Algunos de los riesgos se indican en la Tabla 1.

Tabla 1. Lista de riesgos significativos para el proyecto

\begin{tabular}{|c|c|c|c|}
\hline $\begin{array}{c}\text { Tipo de } \\
\text { Riesgo }\end{array}$ & Descripción & Manejo del Riesgo & Severidad \\
\hline Científico & $\begin{array}{c}\text { Diseño de hardware y } \\
\text { arquitectura de software no } \\
\text { totalmente compatible }\end{array}$ & $\begin{array}{c}\text { Intensificar la coordinación de tareas } \\
\text { con personal de los institutos durante el } \\
\text { primer año de actividad }\end{array}$ & Media \\
\hline Científico & $\begin{array}{c}\text { La operación de la } \\
\text { infraestructura es muy } \\
\text { compleja }\end{array}$ & $\begin{array}{c}\text { Promover la implementación de } \\
\text { prototipos de mayor popularidad y } \\
\text { poder identificar las complejidades y } \\
\text { eventualmente rediseñar }\end{array}$ & Media \\
\hline Tecnológico & $\begin{array}{c}\text { Los resultados de las } \\
\text { simulaciones no reflejan la } \\
\text { realidad }\end{array}$ & $\begin{array}{c}\text { Realizar los cambios necesarios por } \\
\text { métodos en paralelo y realizar los } \\
\text { cambios necesarios }\end{array}$ & Media \\
\hline Funcional & $\begin{array}{c}\text { Objetivos específicos } \\
\text { desviados de lo planificado }\end{array}$ & Realización reuniones regulares & Baja \\
\hline Logístico & $\begin{array}{c}\text { Tareas desarrolladas de } \\
\text { manera desigual }\end{array}$ & $\begin{array}{c}\text { Ajustar coordinación de actividades por } \\
\text { medio de reuniones periódicas }\end{array}$ & Baja \\
\hline
\end{tabular}




\begin{tabular}{|c|c|c|c|}
\hline Logístico & $\begin{array}{c}\text { Las actividades no atraen } \\
\text { atención de la comunidad } \\
\text { científico-académica }\end{array}$ & $\begin{array}{c}\text { Los directores inician contactos con las } \\
\text { comunidades académicas e industriales } \\
\text { que correspondieren }\end{array}$ & Baja \\
\hline Funcional & $\begin{array}{c}\text { La infraestructura no es bien } \\
\text { recibida por el público }\end{array}$ & $\begin{array}{c}\text { Coordinar actividades de divulgación } \\
\text { de manera de mejorar el nivel de } \\
\text { conocimiento y confianza }\end{array}$ & Baja \\
\hline
\end{tabular}

La tarea considerada fundamental para el éxito del proyecto en su conjunto es la transferencia del know-how de los colegas alemanes de manera de comprender e interpretar la arquitectura del hardware y el software de los nodos sensores de la red a instalar, para luego aprender desde las experiencias propias las prevenciones necesarias para la operación de esta tecnología en el ámbito de la selva misionera.

La instalación, configuración y emplazamiento de la red de sensores en el lugar geográfico determinado, se realizará por personal de Universidad Nacional de Misiones, en la locación a determinar con las autoridades del Gobierno de la Provincia de Misiones, y los administradores provinciales y/ó federales de la Reserva Biósfera Yabotí.

Dado que la aplicación informática a instalar en cada uno de los equipos debe ser capaz de transmitir toda la información de estado que se colecte en los sensores, resulta importantísima la experiencia de los integrantes de la Universidad de Lübeck y Universidad Nacional de La Plata, cuya capacidad y sapiencia en el tema aseguran el éxito en esta parte del proyecto.

Un inconveniente que plantea un desafío es lo concerniente a la interconexión de la red de sensores a la infraestructura de la red fija. Fundamentalmente la definición de la puerta de enlace a Internet, dado que la infraestructura de red en la reserva y en toda la provincia de Misiones es mínima.

Instalada y puesta en marcha la red, ésta debe ser operada. Si bien se espera que la red sea bastante autosuficiente en cuanto a organización (función esperada de una red de sensores), es obvia la necesidad de realizar controles periódicos sobre el hardware y los programas instalados.

\section{Materiales}

Se ha planificado la instalación de sesenta nodos para monitoreo ambiental en la Reserva Biósfera Yabotí, los que se integrarán a los aproximadamente dos mil a ser instalados en distintos puntos de Europa. Para ello se ha solicitado la financiación del Ministerio Federal de Educación e Investigación de Alemania y al Ministerio de Ciencia, Tecnología e Innovación Productiva de Argentina ${ }^{15}$, donde hemos participado en una convocatoria abierta de proyectos binacionales. La financiación solicitada incluye solamente la movilidad de los científicos alemanes y argentinos. La contraparte alemana, teniendo en cuenta diferencias en las condiciones, ha solicitado al $\mathrm{BMBF}^{16}$ la provisión de un set de 15 nodos inalámbricos con los dispositivos sensores correspondientes. El ITM proveerá del software middleware necesario, el que podrá ser obtenido desde el sitio repositorio de WISEBED.

\footnotetext{
${ }^{15}$ http://www.mincyt.gov.ar/coopinter_archivos/bilateral/proyectos_2008_BMBF-MINCYT.pdf

${ }^{16} \mathrm{BMBF}$; Bundesministerium für Bildung und Forschung.
} 
La Universidad Nacional de Misiones aportará al proyecto 45 equipos de sensores que se integrarán a los provistos por el ITM, puntos de acceso 802.11 y equipos informáticos de manera de establecer la infraestructura de red.

Entre las empresas proveedoras de tecnología, son ofrecidas varias plataformas WSN orientadas al uso académico y comercial. Algunos de ellos están basados en controladores Atmel AtMega 128 con radiofrecuencias de poco ancho de banda (MICA2 [8]), o de alta tasa de transferencia radial IEEE (MICAz [9], IRIS [7]). Estos pueden ser combinados con una serie de sensores. El TelosB [10] combina una veloz radio con un controlador TI MSP 430 con sensores incluidos. Se podría realizar aquí un largo listado del equipamiento disponible en plaza, lo que es altamente variable con el tiempo dada la velocidad de evolución de la tecnología, por lo que estimamos no amerita explayarnos en este momento del proyecto.

Una vez obtenidas las garantías de los fondos, por parte del BMBF y los de aquellos comprometidos por la contraparte argentina, se delegará en el ITM la adquisición del hardware más actualizado en ese momento.

\section{Impactos y Beneficios Esperados}

\subsection{Impactos Potenciales}

Desde la perspectiva de los sistemas distribuidos, estas infraestructuras interconectadas permiten la investigación en una escala mucho mayor, y debido a su estructura heterogénea se obtienen resultados más representativos. Con ello se establece un nuevo paradigma respecto a las estructuras distribuidas y autoorganizadas.

Desde la perspectiva exterior de las redes, se pretende mostrar una nueva manera de combinar estructuras distribuidas de dispositivos inalámbricos vía Internet, demostrando como este tipo de estructuras trabajan en la dirección de la Internet del futuro.

Para el área interdisciplinaria de las redes de sensores inalámbricos, estas infraestructuras establecerán los cimientos de una visión generalizada del hardware, software, algoritmos y datos; permitiendo un nuevo tipo y calidad de colaboración, marcando probablemente un punto de inflexión de las soluciones individuales a aquellas integradas a gran escala.

A partir de lo anterior, podemos decir que el establecimiento de esta infraestructura de red, mostrará una forma de integración de sistemas distribuidos a diferentes niveles.

Entonces el impacto esperado por la realización del proyecto es "la investigación experimental a largo plazo sobre nuevos paradigmas y entrenamiento práctico en redes avanzadas para la Internet del futuro". Es de esperar que este tipo de proyectos lleguen a ser el punto de condensación para futuros trabajos sobre sensores inalámbricos.

En una escala meno, pero no por ello menos importante para las Universidades Argentinas, se esperan como resultado del presente trabajo:

- Investigadores que puedan testear sus algoritmos a gran escala.

- Evaluación de algoritmos en diferentes escenarios.

- Posibilidad de validar sus sistemas y evaluar rendimientos y comportamientos.

- Posibilidad de acelerar la fase de desarrollo por la utilización de herramientas a ser compartidas. 
- Espacio de comparación de distintas performances comparadas con soluciones existentes (estudio comparativo).

- Entrenamiento de doctorandos e investigadores en la aplicación de una metodología unificada para la teoría y la práctica.

- Evaluación de algoritmos en modelos más realistas, resultando más creíbles.

- Reducción de costos de evaluación de algoritmos para el mundo real.

- Expansión de las redes de sensores a escalas aún mayores.

\subsection{Beneficios Esperados por las Partes}

El acceder a datos del mundo real es una cuestión poco usual en los proyectos actuales de redes de sensores; esta característica ha impulsado a los investigadores alemanes a participar en la elaboración del proyecto presentado a ambos ministerios federales tanto de Argentina como de Alemania.

La oportunidad de desarrollo, apropiación y aplicación de estas novísimas tecnologías en el campo de las TIC's que están siendo desarrolladas y aplicadas en Europa en la actualidad; como de la posibilidad real de tranferencia directa del know-how en este rápido mundo emergente del campo de las redes de sensores inalámbricos, es de una oportunidad estratégica importantísima para Universidades como La Plata y Misiones. En este proyecto en particular, se podrán convalidar nuevas tecnologías y técnicas para la protección y monitoreo de sus recursos naturales.

\section{Referencias}

[1] WISEBED, "Information and Communication Programme". http://www.wisebed.eu/ 2008.

[2] FUTURO, "Future Internet". http://www.future-internet.org/, 2008.

[3] LINTI, Laboratorio de Investigación de Nuevas Tecnologías Informáticas, Universidad Nacional de La Plata, http://www.linti.unlp.edu.ar

[4] ITM, Institut für Telematik, Universität zu Lübeck, http://www.itm.uni-luebeck.de.

[5] FCEQyN, Facultad de Ciencias Exactas, Químicas y Naturales, Universidad Nacional de Misiones. http://www.fceqyn.unam.edu.ar/

[6] INFO, Secretaría de Posgrado, Facultad de Informática, Universidad Nacional de La Plata. http://postgrado.info.unlp.edu.ar/

[7] Crossbow IRIS 2.4GHz.

http://www.xbow.com/Products/productdetails.aspx?sid=264.

[8] Crossbow MICA2 868, 916 MHz.

http://www.xbow.com/Products/productdetails.aspx?sid=174.

[9] Crossbow MICAz 2.4GHz.

http://www.xbow.com/Products/productdetails.aspx?sid=164.

[10] Crossbow TelosB. http://www.xbow.com/Products/productdetails.aspx?sid=252. 


\title{
Twitter, Soporte de una Red de Sensores Inalámbricos
}

\author{
E.O. Sosa, Student Member IEEE; S. Fischer, Non Member; F.J. Díaz, Associate Member, IEEE; \\ A.A. Arrieta , Non Member; S.F. Vier, Non Member; A. S. Fischer, Non Member; J.P. Sevi, Non Member
}

\begin{abstract}
Resumen - Se ha utilizado la infraestructura de Twitter como soporte para la publicación de datos capturados y procesados por una red de sensores inalámbricos (WSN). La creciente necesidad de vínculos que aseguren un nivel de servicios para proyectos que involucren a las WSN en lo referente a medio ambiente, forestoindustria y agricultura en zonas de Argentina, ha conllevado a una prueba de concepto y test de los diferentes componentes de hardware y software para utilizar a Twitter como alerta ante eventos identificados en algunos fenómenos. Como resultado se han publicado y comunicado eventos detectados por la red de sensores en forma instantánea, lo que demuestra ser una alternativa eficaz a la necesidad de soporte de infraestructura
\end{abstract}

Temas claves - Red de Sensores Inalámbricos, WSN, Twitter, Python, Telemonitoreo, Sistemas de procesamiento distribuidos, redes ad hoc

\section{INTRODUCCIÓN}

I A utilización de las redes sociales ha alcanzado un Lesarrollo que no se discute a esta altura de las circunstancias. Una red social está constituida por agrupaciones de individuos, de acuerdo a intereses comunes. Estas redes son de tipo presencial ó virtual. En el último de los casos nos referimos a éstos como sitios web sociales.

Twitter es un servicio de red social y servicio de microblogging que permite a sus usuarios enviar y leer microentradas de texto de una longitud máxima de 140 caracteres. Estos mensajes son denominados "tweets". El envío de estos mensajes se puede realizar tanto por el sitio web de Twitter, como vía SMS desde un teléfono móvil, desde programas de mensajería instantánea; o incluso desde cualquier aplicación desarrollada por terceros.

Estas actualizaciones se muestran en la página de perfil del usuario, y son también enviadas de forma inmediata a otros usuarios que han elegido la opción de recibirlas, a quienes se los conoce como seguidores. El envío de estos mensajes puede restringirse ya sea, sólo a miembros de su círculo de usuarios

E.O. Sosa es profesor asociado en la Facultad de Ciencias Exactas Químicas y Naturales de la Universidad Nacional de Misiones, Félix de Azara 174, Posadas, Argentina (e-mail: es@fceqyn.unam.edu.ar)

S. Fischer es director del Instituto de Telemática de la Universidad de Lübeck, Alemania (email: fischer@itm.uni-luebeck.de)

F.J. Díaz es profesor titular en la Facultad de Informática de la Universidad Nacional de La Plata, Argentina (e-mail: jdiaz@info.unlp.edu.ar)

A.Arrieta S. Vier, J. Sevi y A. Fischer integran del proyecto "Hacia una red global de Sensores“ de la Facultad de Ciencias Exactas Químicas y Naturales de la Universidad Nacional de Misiones, Félix de Azara 174, Posadas, Argentina (e-mail:[aarrieta, sergiovier, pablosevi, andresfischer] @ gmail.com]. seleccionados, ó permitir el acceso a la información al universo de usuarios Twitter. Desde su creación en 2006 ha ganado popularidad en todo el mundo y actualmente tiene más de 100 millones de usuarios.

El logo del sitio de Twitter indica "Descubre lo que está ocurriendo en este momento, en cualquier lugar del mundo [1]". Lo anterior ha disparado nuestra inquietud en verificar si la infraestructura ubicua de esta red social es capaz de soportar una aplicación de redes de sensores.

Existe una realidad en la República Argentina en lo que respecta a probabilidades de obtener una cobertura eficiente y eficaz en lo que se refiere a telefonía y/ó el consecuente acceso a la red digital soporte, como infraestructura para instalaciones típicas de las redes de sensores inalámbricos. La variedad de proveedores y posibilidades de acceso a servicios de calidad por medio de un Contrato de Nivel de Servicios (SLA), es una tarea bastante complicada si el proyecto se encuentra fuera del triángulo geográfico formado por las ciudades de Mendoza, Santa Fé y Bahía Blanca. Esta complicación se presenta esencialmente como carencia del servicio por parte de la prestadora, ó costos excesivamente elevados de implementación de una solución particular.

Lo anterior amenaza la expansión de proyectos de WSN que serían vistos de buen modo y aceptados por la forestoindustria, la agricultura y ganadería; como así todo lo que tenga que ver con contralores ambientales en la zona y el país todo.

La red de telefonía celular se encuentra mucho más avanzada en ese sentido, por lo que permitiría cubrir el déficit nombrado anteriormente.

Se ha pretendido utilizar las características propias de las redes de sensores inalámbricos y el hecho de instantaneidad de la red social elegida. Twitter es una plataforma de envíos de mensajes no dependientes del equipamiento [2], manera elegante de decir que es capaz de enviar y recibir mensajes por una variedad de equipos, de manera instantánea.

La utilización de Twitter como una herramienta de notificación de eventos identificados como importantes, no es algo nuevo. En el Japón se ha elaborado un algoritmo de procesamiento de los mensajes [3], con el cual se pueden anunciar con suficiente antelación, y una alta probabilidad, la ocurrencia de terremotos en todo el país; aún antes que sea notificado a la población por la Agencia Meteorológica Japonesa. En cuestiones ambientales se realizan mediciones de valores de referencia, y en caso de superación de los límites considerados tope, se emite una alerta de notificación por 
Twitter[4]. En forma idéntica se realizan tareas de prevención de incendios forestales [5].

Si bien existen diversos tipos de sensores, la mayoría de ellos son eléctricos ó electrónicos. Un buen sensor debe obedecer a las siguientes reglas: a) debe ser sensitivo a la propiedad a ser medida, b) debe ser no sensitivo a las otras propiedades y c) no debe influenciar a la propiedad medida.

Las redes de sensores, inalámbricos y distribuidos, posibilitan infinitas aplicaciones de medición y control del mundo físico. Estos equipos son reconocidos también como nanocomputadoras [6]. Se utilizan en la medición de temperatura, sonido, presión, movimiento, contaminantes, y muchos otros parámetros. Generalmente son económicos y pequeños, de manera que se instalan y producen en un número elevado.

Las WSN son instalaciones que capturan y diseminan los datos a través de la misma. La idea primigenia de estas redes de sensores ha nacido del desarrollo de de una serie de nodos por parte de la $\mathrm{UCB}^{1}$ a los que denominaron nodos Mica [7]. Debido a partes de hardware poco confiables en estos equipos, y como forma de superar las imperfecciones, se evoluciona a los nodos Mica2, Mica2Dot and MicaZ. El Mica2 es el sucesor de los nodos Mica y proporciona una mejor interfaz de radio y diseño [8]. The Mica2Dot es bastante más pequeño que Mica2 y su precio es menor. El nodo MicaZ [9] se caracteriza porque reemplaza el sistema de radio propietario por otro compatible del tipo IEEE 802.15.4. Como sucesor de Mica2 aparece el nodo Telos [10],[11] en 2004. Este nodo se orienta fundamentalmente a atacar una de las debilidades de la tecnología, el consumo de energía, cortos tiempos de wake-up y la utilización de radio IEEE 802.15.4 . Subidos a la ola paradigmática, se desarrollaron en Europa varias plataformas. Se pueden destacar varias de estas tecnologías. El BTnode [12], [13] que proporciona conexión a notebooks, PDAs y teléfonos celulares por Bluetooth. La F.U. Berlín, que ha desarrollado y establecido el ESB (Embedded Sensor Board) [14] que es utilizada como plataforma de educación e investigación. Luego el ESB evolucionó a la marca comercial Scatternode. Demostrando el interés y desarrollo del campo, la plataforma iSense [15] se desarrolla en Alemania, en un todo de acuerdo a los estándares comunes de la tecnología.

\section{MATERIALES Y MÉTODOS}

\section{A. Hardfware iSense}

Como plataforma base para el desarrollo de las tareas se han utilizado equipos con un módulo principal iSense. El dispositivo integra un procesador Jennic JN5139 con un sistema radial de $2,4 \mathrm{GHz}$ compatible con normas IEEE 802.15.4, con ancho de banda de 250kbit/s, sistema de encriptación por hardware $\mathrm{AES}, \mathrm{ToF}^{2}$ para cálculo de distancias entre nodos, 192kB de ROM, 96kB of RAM, así como una variada posibilidad para la utilización de periféricos

\footnotetext{
${ }^{1}$ UCB: Universidad de California en Berkeley.

${ }^{2}$ Time of Flight
}

analógicos y digitales. En la figura 1 se presenta al módulo principal iSense.

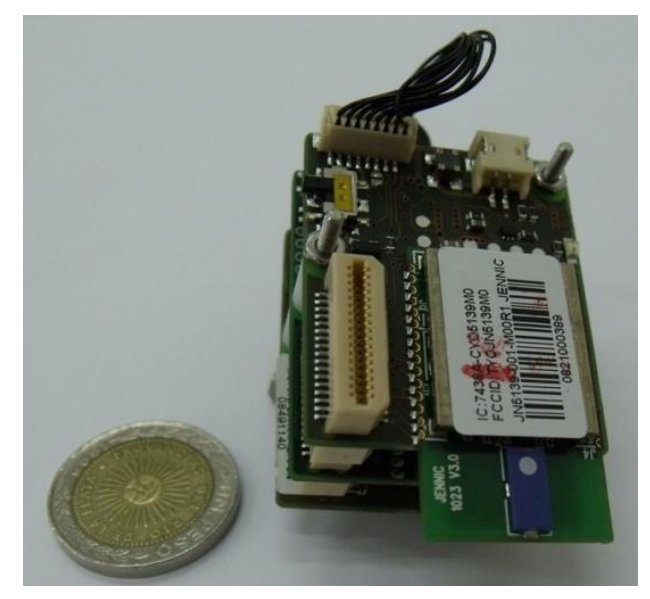

Figura 1. Módulo principal iSense

\section{B. Herramientas y Software iSense}

En una aplicación típica de redes de sensores, el observador está interesado en el seguimiento fenómenos bajo ciertas restricciones de latencia y la precisión. En una típica red de sensores, cada uno de los equipos individuales realiza la medición de valores locales, y difunde esta información hacia los otros miembros de la red en caso de necesidad; y, eventualmente, al observador. Las medidas realizadas por los diferentes sensores son muestras discretas del fenómeno físico bajo observación, que dependerá tanto de la precisión y exactitud de los sensores; como de la ubicación de los mismos.

El hardware iSense se proporciona junto a un firmware operativo y de red modular. Éste permite la generación de aplicaciones pequeñas, pero completas, y provee una base sólida para el desarrollo rápido de aplicaciones. Brinda una API C++ para el nodo hardware, funcionalidades de sistema operativo y una amplia variedad de protocolos de red.

El sistema de software iSense [15] incluye un número variado de servicios y protocolos listos para usar, tales como encaminado, sincronización de tiempo, programación "en el aire", etc.

Como pasarela a la red Twitter se ha utilizado una computadora personal de escritorio con sistema operativo Debian GNU/Linux con conexión directa a Internet. Se utilizaron otras computadoras personales como plataformas de desarrollo de las aplicaciones que luego serían transferidas a los dispositivos sensores. En estos equipos se debieron instalar los paquetes que permitieran desarrollar las aplicaciones en $\mathrm{C}++\mathrm{y}$ compilarlas de tal manera de poder luego distribuirlas a los diferentes nodos. En los equipos de desarrollo se utilizó una plataforma PC+Linux en sus distribuciones Ubuntu y Debian. Se instalaron las aplicaciones necesarias tales como: make, cmake and $\mathrm{g}++$. Esta plataforma requiere ineludiblemente de un compilador $\mathrm{C}++$ que asegure la integración perfecta con las librerías. Se ha instalado el compilador ba-elf-g++[15], específicas para nodos que presenten los controladores Jennic. 
En la necesidad de utilizar un entorno de desarrollo integrado, hemos elegido a la herramienta de código abierto Eclipse $^{3}$, que consiste en un editor de código, un depurador y un constructor de interfaz gráfica. Eclipse es un ambiente de desarrollo de software multilenguaje que incluye un IDE y un sistema expandible de plugins.

Para realizar la conexión con los sensores en el período de desarrollo y pruebas, se utilizó el software iShell, que también forma parte de las herramientas de la plataforma iSense. En la figura 2 Se observa captura de una pantalla de iShell.

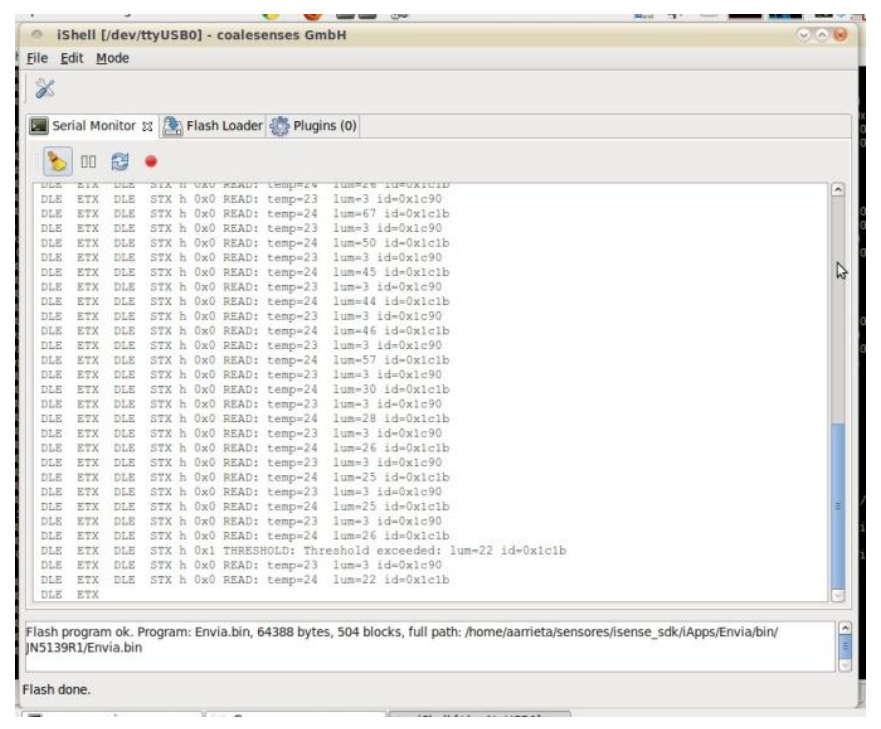

Figura 2. Captura de pantalla de iShell

A través de la pantalla monitor de iShell se puede hacer un control de los mensajes de debug provenientes del sensor, que son capturados y leídos desde los dispositivos a través de una conexión serie o USB. Además, permite realizar la instalación del archivo binario en cada uno de los sensores. Este archivo binario es la compilación del programa de código conjuntamente con las librerías, y se convierte en el programa ejecutable en cada sensor. La herramienta iShell es la contraparte del firmware de operación y de red iSense. Más allá de la variedad de funciones habilitadas para la operación de las redes de sensores inalámbricos iSense, iShell puede ser fácilmente expandida con plugins desarrollado por los usuarios de acuerdo a las necesidades que se presenten.

En la figura 3 se muestra el módulo gateway para ser conectado a una PC vía USB, que alimenta al sensor de energía.

\section{A. Servicio Twitter}

Para el acceso y autenticación a una cuenta, Twitter utiliza el protocolo abierto de autenticación OAuth[16] aplicado sobre protocolo http. OAuth proporciona un procedimiento para acceder a los recursos de un servicio en nombre de un propietario del recurso (tal como un cliente diferente o un

\footnotetext{
${ }^{3}$ http://www.eclipse.org/
}

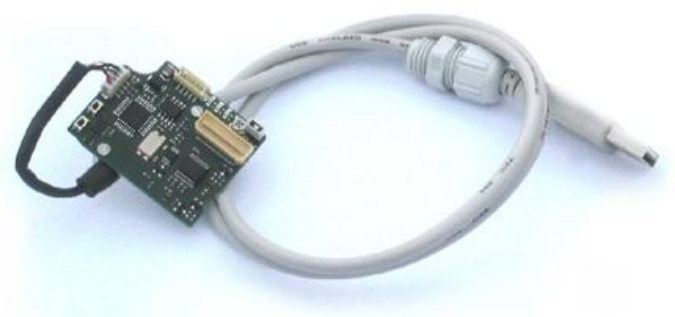

Figura 3. Modulo Gateway con conector USB

usuario final). Asimismo proporciona un proceso para autorizar el acceso de terceros a los recursos de su servidor sin compartir sus credenciales (normalmente, un nombre de usuario y contraseña), utilizando redirecciones user-agent.

Twitter ofrece la posibilidad de obtener una ficha de acceso único. Mediante el uso de dicha ficha, se elimina la necesidad de implementar todas las características de OAuth.

\section{IMPLEMENTACIÓN}

La arquitectura de la experiencia, se conforma de diversos elementos, tanto hardware como software. Se pueden diferenciar dos secciones bien definidas: la red de sensores inalámbricos y la interfaz de conexión hacia Internet. La figura 4 muestra la arquitectura implementada.

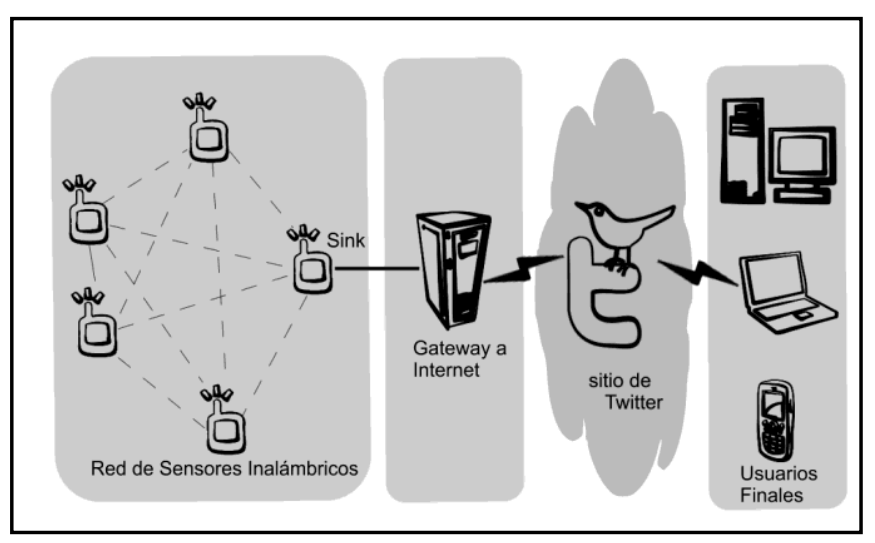

Figura 4.Arquitectura WSN implementada

\section{A. Red inalámbrica de Sensores}

Conformada por equipos iSense. Han sido utilizados tanto el módulo ambiental como el modulo de estación meteorológica provisto por Coalesenses[15]. Se han monitoreado valores de presión atmosférica, temperatura, luminosidad y la humedad del aire.

Con este fin se ha desarrollado una rutina de captura de eventos y generado un texto específico de salida de manera de poder filtrarlo y/o catalogarlo en etapas posteriores. Se ha programado a los sensores para que indiquen por medio de encendido de LEDs específicos cuando se encuentran en modo medición

Ha sido necesario programar el firmware de dichos equipos para que cumplan con las necesidades de la implementación. Al programarlos se los ha configurado para que realicen una lectura de datos cada diez minutos, evitando así un excesivo 
tráfico de datos entre el Gateway y Twitter. En caso de variaciones bruscas en las variables medidas, los sensores han sido programados para que emitan un aviso.

La lectura de los sensores es enviada al resto de los nodos de la red por difusión utilizando la interfaz de radio de cada dispositivo sensor. Tanto al leer como al recibir datos, cada nodo los imprime en su salida serial

Con el objeto de simplificar la tarea de programación, y hacer más sencillo el reemplazo de nodos o el agregado de nuevos nodos a la red, se ha programado que todos los nodos realicen la misma tarea de lectura y envío de datos. Esto implica cierta redundancia en la operación, pero refleja la flexibilidad de este tipo de redes y cómo puede adaptarse su topología sin alterar su implementación.

\section{B. Interfaz de conexión hacia Internet}

Para publicar en Twitter, se ha utilizado uno de los nodos de la red como sink (sumidero), conectado a través del puerto USB a la computadora que actúa de servidor. Se ha instalado en la misma un software capaz de leer los datos que el dispositivo genera en su salida serial.

Para esa tarea, se ha utilizado un script hecho en el lenguaje Python ${ }^{4}$, que permite conectarse a dispositivos seriales y realizar operaciones sobre los mismos. A dicho script se le hicieron algunas modificaciones para incorporar la funcionalidad de publicar los datos en la cuenta de Twitter.

Existen varias librerías para el lenguaje Python, que proveen una interface completamente probada para trabajar con el protocolo OAuth, tanto en sistemas clientes como servidores. Una de ellas es python-oauth2, el que se ha utilizado en este trabajo para la validación de envíos a Twitter. El envío de la información se publica en Twitter.com tanto cuando se alcanza un tiempo prefijado establecido en $10 \mathrm{~min}$, como al alcanzar un límite establecido como disparador del proceso (threshold). En el Apéndice A se muestra la porción de código relacionado a la autenticación y envío de los datos.

\section{Resultados ObTENIDOS}

Utilizando las tecnologías descriptas anteriormente, se ha logrado realizar el despliegue experimental de una red de sensores cuyas mediciones fueron publicadas en Twitter.

Este despliegue de equipamiento se ha limitado no solamente al laboratorio, sino también a escala piloto. La distancia entre cada uno de los nodos ha sido entre los quince y ochenta metros.

Los desafíos técnicos del proyecto pudieron ser solucionados, sirviendo como primer paso hacia nuevas investigaciones relacionadas con el desarrollo e implementación de redes de sensores inalámbricos.

Twitter ha demostrado ser capaz de servir como soporte a una red como la planteada en el presente trabajo. En la ilustración 2 se muestra reportes publicados desde la WSN accedido vía telefonía celular en un vehículo en movimiento.

\footnotetext{
${ }^{4}$ http://pyserial.sourceforge.net/examples.html\#miniterm
}

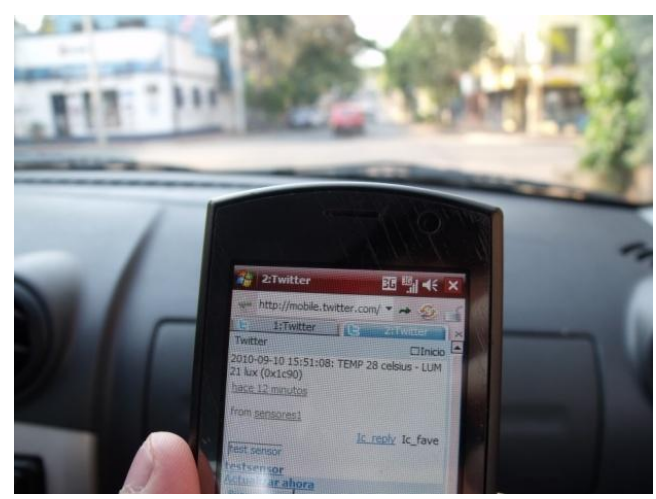

Ilustración 2. Datos de la WSN accedidos del sitio de Twitter vía telefonía celular en un vehículo en movimiento.

En la Ilustración 3 se muestran los reportes en el sitio Twitter accedidos desde un navegador, pudiendo observarse las distintas direcciones MAC de los emisores de la información reportada. Los sensores en la ilustración reportan la temperatura ambiental en ${ }^{\circ} \mathrm{C}$ y la intensidad luminosa en Lux.

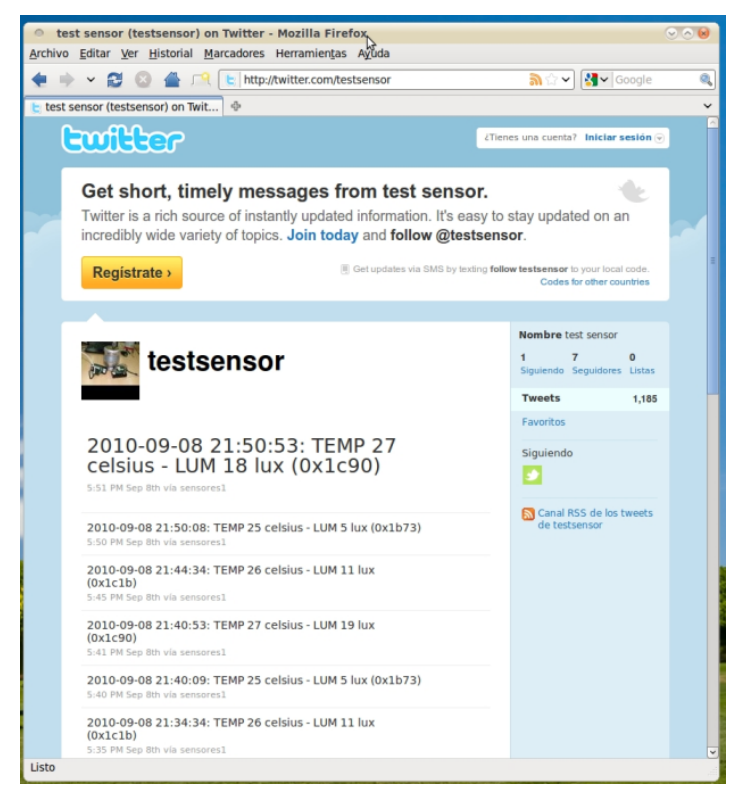

Ilustración 3 Reportes de la WSN en el sitio web de Twitter

\section{CONCLUSIONES}

El trabajo realizado permite extender el uso de la aplicación de WSN en nuestro país a lugares sin acceso a Internet, o con accesos sumamente restringidos, utilizando para ello la red de celulares y mediante SMS (de bajo costo). Esto es fundamental al momento de proyectar instalaciones en muchas regiones de Argentina.

La integración con Twitter no solo permite colectar la información, sino que se convierte en una forma de publicar, sociabilizar y transparentar información, que de otra manera es de difícil acceso. Uno de los resultados importantes para las 
WSN tiene que ver con la generación de alertas tempranas en distintas situaciones, de acuerdo a necesidades diferentes.

Los resultados obtenidos han demostrado ser valederos para la experiencia piloto realizada. Ha demostrado un grado de integración y escalamiento que ameritan ser tenidos en cuenta para proyectos de contralor de una variedad de eventos, ya que consiste en una tecnología que no requiere una infraestructura de comunicaciones propia, utiliza una API para publicar sumamente cómoda, y con un mecanismo de acceder a los datos publicados por demás simple (por navegador o celular).

Por último, comprobado que Twitter está actualmente integrado a múltiples servicios en Internet, se habilitan nuevas aplicaciones para las redes de sensores clásicas.

\section{TRABAJo Futuro}

Los experimentos realizados nos han permitido establecer un mecanismo de difusión de los datos que son recolectados desde una red del tipo WSN. Aunque se ha experimentado únicamente con el servicio de Twitter, otras redes también pueden ser utilizadas. Una tarea futura será identificar qué otras redes pueden brindar el mismo servicio que Twitter, analizar sus ventajas y puntos débiles; y en caso de ser una plataforma viable, finalmente adaptar el código para usar dicha red. Entre las alternativas se encuentran las diferentes redes sociales (Facebook, Buzz), servicios de blogs (Blogger, Wordpress), e incluso la posibilidad de montar una infraestructura propia para tal fin.

El mecanismo implementado, que interconecta un nodo sensor con un servidor, permite una comunicación bidireccional entre ambos. Esto abre la posibilidad a desplegar servicios en sentido inverso al experimento, es decir, que se puedan enviar comandos hacia los nodos sensores para lograr una respuesta desde éstos.

Otro de los trabajos futuros constituye el tratamiento de la información generada. Con el experimento actual nos centramos en enviar los datos hacia Internet y hacerlo accesibles desde cualquier lugar del mundo. En una implementación real de una red de sensores (fuera del laboratorio), el hecho de poder manipular los datos obtenidos es sumamente importante. Se debe investigar la manera de trabajar sobre la API proporcionada por la plataforma para la descarga de los datos; como así también el tratamiento de los mismos mediante algoritmos de análisis. Un objetivo clave aquí puede ser el análisis de la información para la detección de alertas (la lógica sobre tal procedimiento ha de estar fuera del sensor y ser independiente de su implementación).

\section{AGRADECIMIENTOS}

A las autoridades del Parque Tecnológico Misiones (PTMi) y a la Incubadora de Empresas de Bases Tecnológicas por soportar al proyecto en sus instalaciones, proporcionando la infraestructura necesaria.

\section{APENDICE A}

Líneas de código Phyton donde se envían los datos al sitio Twitter.

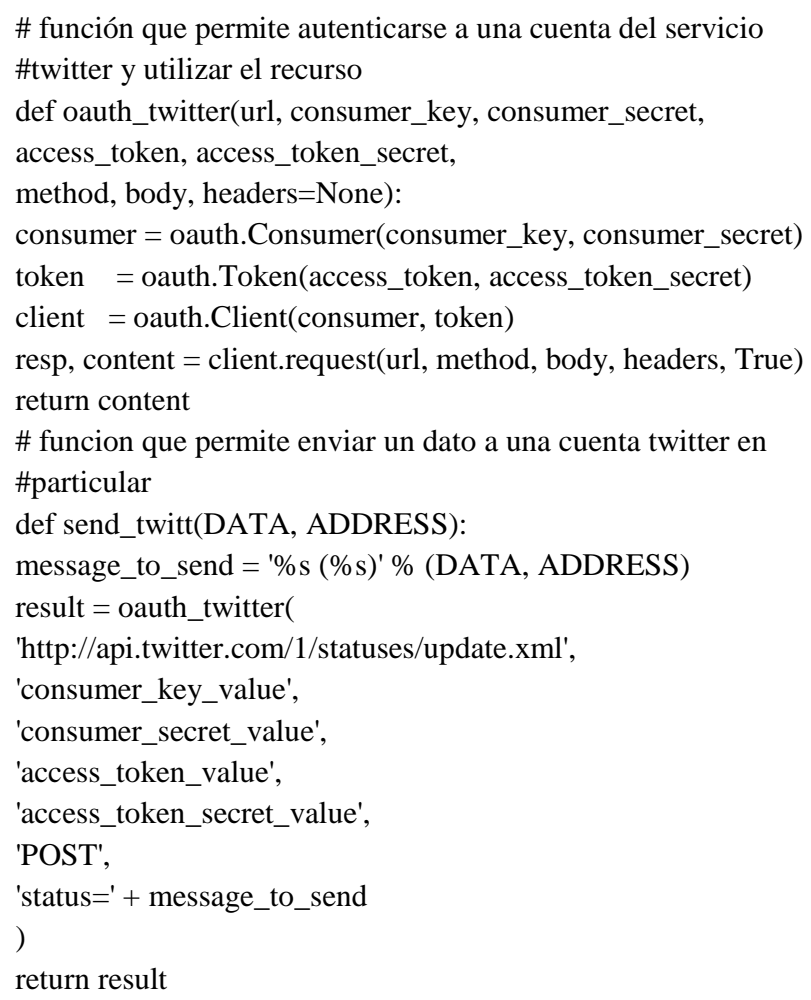

\section{REFERENCIAS}

[1] Twitter. [En línea] http://twitter.com/.

[2] Sarah Milstein, with Abdur Chowdhury, Gregor Hochmuth, Ben Lorica, Roger Magoulas. Twitter and the Micro-Messaging Revolution. s.l. : O’Reilly Media, Inc, 2008. [En Línea] http://www.weigend.com/files/ teaching/haas/2009/readings/OReillyTwitterReport200811.pdf

[3] Sakaki, Takeshi, Okazaki, Makoto and Matsuo, Yutaka. Earthquake Shakes Twitter Users: Real-time Event Detection by Social Sensors. Raleigh, North Carolina : WWW2010 . [En Línea] Septiembre2, 2010 http://ymatsuo.com/papers/www2010.pdf

[4] Gardens, Open. Mobile Web 2.0 for sensor networks: Interfacing Sensor Networks Twitter and WordPress. [En Línea] Agosto 5, 2010. http://www. opengardensblog.futuretext.com

[5] Sobrera, Javier. Detecting Forest Fires using wireless sensor Networks with Waspmotes. [En Línea] Abril 9, 2010. http://www.libelium.com /libeliumworld/articles/101031032811.

[6] Li, Xianjang. Wireless Ad Hoc and Sensor Networks. Cambridge, Cambridge University Press, 2008. Pag. 12

[7] Jason L. Hill, David E. Culler . Mica: A Wireless Platform for Deeply Embedded Networks. Berkeley, CA : IEEE Micro, 2002. Pag. 12-24

[8] Szewczyk, R., Mainwaring, A., Polastre, J., Anderson, J., and Culler, D. An Analysis of a Large Scale Habitat Monitoring Application. Proc 2 SenSys'04. Baltimore, Maryland, USA. Pag 12-24

[9] Inc., Crossbow Technology. MICAz wireless measurement system. [Online] Junio 2004. [En Línea] http://www.xbow.com

[10] Moteiv Corporation. Tmote Sky. [Online] 2005. http://www.moteiv.com /productstmotesky.

[11] Joseph Polastre, Robert Szewczyk, and David Culler. Telos: Enabling Ultra-Low Power Wireless Research. [Online] Computer Science Department, University of California, Berkeley. [En Línea] http://www.polastre.com/papers/spots05-telos.pdf. 
[13] Beutel, Jan. The BTnode Story. Reflections on Almost a Decade of Mote Class Devices. [Online] 2009 http://www.mics.org/ZH08/slides/s22a.pdf.

[14] Freie Universität Berlin, AG. ScatterWeb Homepage. [Online] http://cst.mi.fu-berlin.de/projects/ScatterWeb/

[15] Coalesenses $\mathrm{GmbH}$. Bridging the gap between virtuality and reality . [Online] 2008. http://www.coalesenses.com/.

[16] Internet Engineering Task Force (IETF) (2010, Abril). Request for Comments: 5849: The OAuth 1.0 Protocol. E. Hammer-Lahav, Ed. [en línea]. Disponible en: http://tools.ietf.org/html/rfc5849

\section{BIOGRAFÍAS}

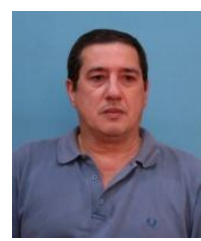

Eduardo Omar Sosa, Recibió el grado de Ingeniero Químico en la Universidad Nacional de Misiones (UNaM) en 1983. Obtuvo su grado académico de Magister en Redes de Datos en la Universidad Nacional de La Plata en el año 2003. Actualmente es profesor asociado en la UNaM y doctorando en Ciencias Informáticas en la UNLP.

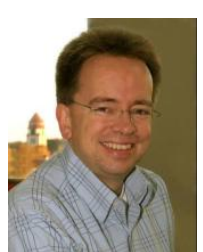

Stefan Fischer, Diplomado en Informática de la Universidad de Mannheim en 1992. Doctorado en informática en 1996, Universidad de Mannheim. 1996/1997 Postdoctorado Universidad de Montreal. Profesor de Tecnología de la Información 1998-2001, la Universidad Inter-nacional Bruchsal. 2001/2004 Profesor en el Instituto de los Sistemas Operativos y Redes Informáticas de la Universidad de Braunschweig. Profesor y Director del Instituto de Telemática de la Universidad de Lübeck

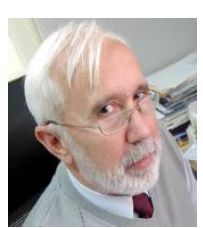

Francisco Javier Díaz, Estudió en la Facultad de Ciencias Exactas de la Universidad Nacional de La Plata(UNLP), graduado de Calculista Científico en 1979 y de Licenciado en Matemática Aplicada en 1984. Es Director del Centro Superior para el Procesamiento de la Información de la UNLP desde 1990, Director del Laboratorio de Nuevas Tecnologías Informáticas desde 1995, Director de la Maestría de Redes de Datos desde 1997. Actualmente es Decano de la Facultad de Informática de la Universidad Nacional de La Plata.

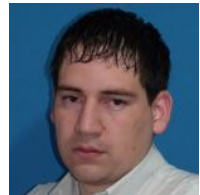

Arnaldo Ariel Arrieta, estudiante de la carrera de Ingeniería en Informática en la Universidad Gastón Dachary, Posadas, Misiones. Se interesa en las redes de sensores inalámbricos.

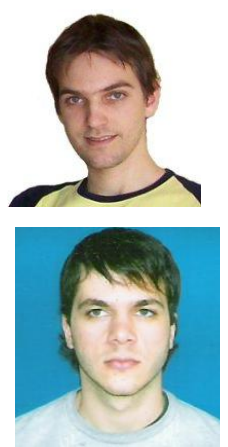

Sergio Fabián Vier, Se graduó de Licenciado en Sistemas de Información en la Universidad Nacional de Misiones en el año 2009; interesado en sistemas modernos de comunicación, Redes de Sensores Inalámbricas, Sistemas distribuidos, Ingeniería de Software.

Andrés Fischer, Estudiante de la carrera de licenciatura en Sistemas de Información en la Universidad Nacional de Misiones. Interesado en simulaciones de sistemas modernos de comunicación.

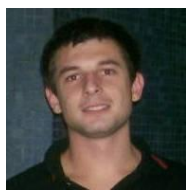

Jose P. Sevi. Estudiante de la carrera de licenciatura en Sistemas de Información en la Universidad Nacional de Misiones. Interesado en redes de sensores 


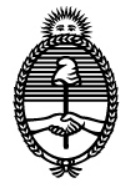

Buenos Aires, 19 de Noviembre de 2008.

Señor Investigador

Dr. Francisco DIAZ

$\underline{\text { S. }}$ L_L.

Tengo el agrado de dirigirme a usted con relación al proyecto "Hacia una Red Global de Sensores Interconectados. Un ensayo experimental Argentino-Alemán”, presentado en el marco del Programa de Cooperación Científico-Tecnológico entre el Ministerio de Ciencia, Tecnología e Innovación Productiva de la República Argentina (MINCYT) y el Bundesministerium für Bildung und Forschung (BMBF) de Alemania.

Al respecto, llevo a su conocimiento que la Reunión de la Comisión Mixta MINCYTBMBF se llevó a cabo el día 14 de Noviembre, resultando seleccionados los proyectos que se ejecutarán durante el bienio 2009-2010.

En este sentido, cumplo en informarle que su proyecto ha sido aprobado y el código asignado es AL0807. Las misiones que serán financiadas por MINCYT y BMBF son las siguientes:

- 1 misión de Argentina a Alemania de 15 días

- 1 misión de Argentina a Alemania de 30 días

- 1 misión de Alemania a Argentina de 15 días

Para solicitar los pasajes y viáticos correspondientes a las misiones aprobadas deberán completarse las Planillas de Solicitud de Pasajes y de Viáticos, que están disponibles en el sitio de la MINCYT en Internet (www.mincyt.gov.ar, Cooperación Internacional) y remitirse por fax, Área Bilateral con SESENTA (60) días de anticipación en el caso de Solicitud de Pasajes y de TREINTA (30) días de Solicitud de Viáticos. Asimismo, deberá tenerse en cuenta que este Ministerio ha dispuesto que no podrán efectuarse viajes en temporada alta, esto es, entre los períodos 01/07 al 27/07 y 5/12 al 20/02 y que las misiones aprobadas deberán iniciarse indefectiblemente antes del 5 de diciembre de 2009.

Ruego a usted tenga en cuenta toda la información consignada precedentemente a efectos de evitar inconvenientes en la correcta ejecución del programa de cooperación.

Sin otro particular, saludo a usted muy atentamente.

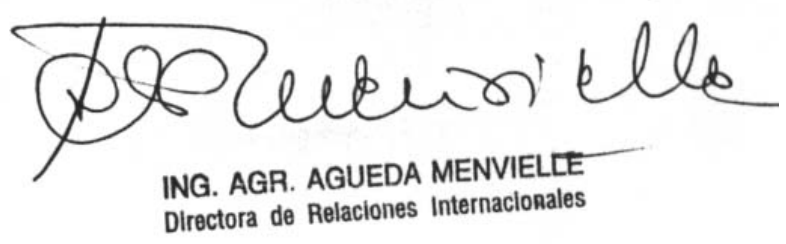


Señor Investigador

Dr. Francisco DIAZ

$\underline{S .}$ D.

Tengo el agrado de dirigirme a usted en relación al proyecto "Hacia una Red Global de Sensores Interconectados. Un ensayo experimental Argentino-Alemán" (Código: AL/08/07), aprobado en el marco del Programa de Cooperación Científico-Tecnológica entre el Ministerio de Ciencia, Tecnología e Innovación Productiva de la República Argentina (MINCYT) y el Bundesministerium für Bildung und Forschung (BMBF) de Alemania.

Al respecto, llevo a su conocimiento que la Reunión de la Comisión Mixta MINCYT-BMBF se llevó a cabo el 14 de diciembre de 2010, y cumplo en informarle que ha sido aprobado el segundo y último año de ejecución de su proyecto. Las misiones que serán financiadas por MINCYT y BMBF durante el año 2011 son las siguientes:

- 2 misiones ARG-AL de 15 días.

- 1 misión AL-ARG de 15 días.

Todos los recursos deberán ejecutarse entre el 1 de enero y el 31 de diciembre de 2011. Como anexo a esta nota se envían las "Condiciones para la Ejecución de Proyectos en Programas de Cooperación Bilateral". Ruego a usted tener en cuenta toda la información allí contenida a efectos de asegurar la correcta ejecución del proyecto.

Sin otro particular, saludo a usted muy atentamente.

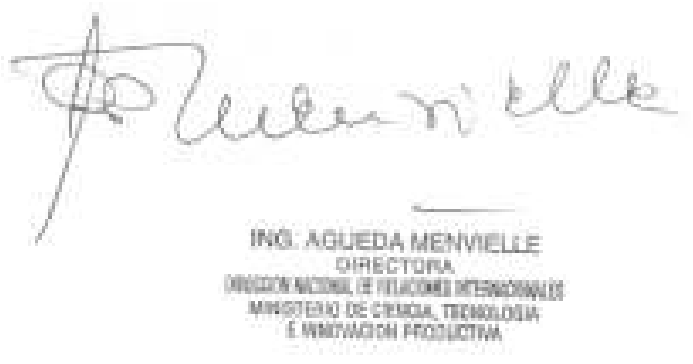


Application Form:

Bilateral Cooperation in Education and Research
International Bureau of the Federal Ministry of Education and Research

PKZ:

LpSys:

$\mathrm{x}$ Preparation of new project

Integration in current project,current project id

Title of proposal (in English)

Towards a Global Sensor Network Interconnection. A German-Argentinean field trial

Title of proposal (german)

Weltweite Sensornetzwerkverknüpfung - ein deutsch-argentinischer Feldversuch

Duration Start

01.01 .2009

End:

31.12 .2010

Deutscher Partner (Principal investigator)

Institution

Type of organisation

Division/Institute

Address (street, POB)

Universität zu Lübeck

\begin{tabular}{|l|l}
\hline Universität Web address http://www.itm.uni-luebeck.de \\
\hline
\end{tabular}

Institut für Telematik

Ratzeburger Allee 160

ZIP code

$23538 \quad$ City

Lübeck

Name

Firstname

Mr./Mrs./Ms. Acad. title

Phone

Fischer

Stefan

Herr

Prof. Dr.

$+494515005380$

E-Mail

Fax

fischer@itm.uni-luebeck.de

$+494515005382$

\section{Foreign partner (Principal investigator)}

Institution

Type of organisation

Division/Institute

Address (street, POB)

ZIP code

Name

Diaz

E-Mail

Universidad Nacional de La Plata

\begin{tabular}{|c|c|c|c|c|}
\hline \multicolumn{3}{|l|}{ University } & Web address & http://www.linti.unlp.edu.ar \\
\hline \multicolumn{5}{|c|}{ Laboratorio de Investigación en Nuevas Tecnologías Informáticas } \\
\hline \multicolumn{5}{|c|}{ Calle 115 entre 49 y 50} \\
\hline 1900 & City & La Plata & Country & Argentinien \\
\hline
\end{tabular}

Firstname

Francisco Javier

jdiaz@unlp.edu.ar Mr./Mrs./Ms. Acad. title

Phone

+542214228241

Fax

+54 2214230124

Project ID: 1475

EWA Version $1.0 \mathrm{CVS} 070522$ 
Application Form:

Bilateral Cooperation in Education and Research

PKZ:
International Bureau of the Federal Ministry of Education and Research

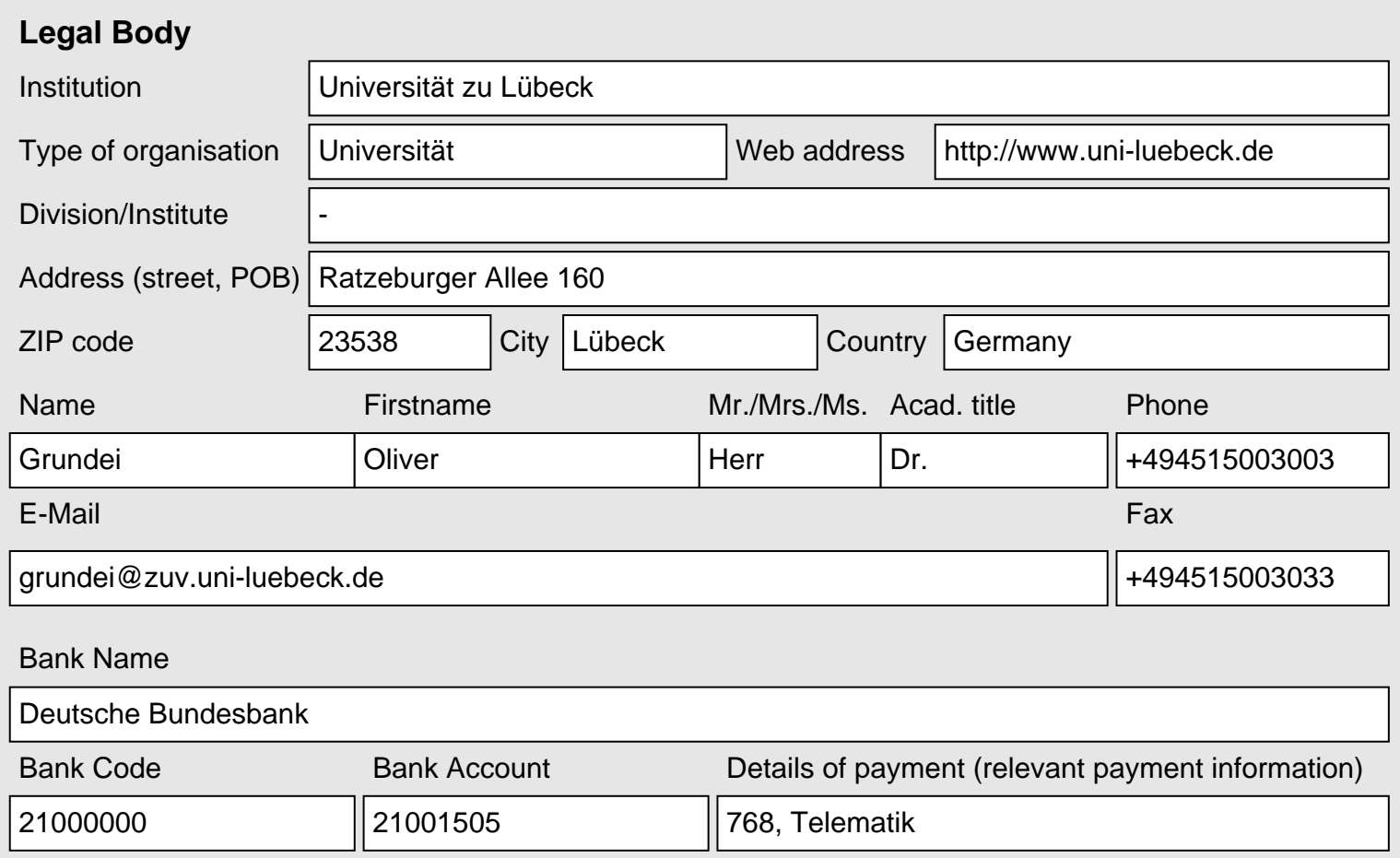


Application Form:

Bilateral Cooperation in Education and Research

PKZ:
International Bureau

of the Federal Ministry

of Education and Research

\section{Other Foreign Partners}

Institution

Universidad Nacional de Misiones

Type of organisation

\begin{tabular}{|l|l|}
\hline University & Web address http://www.fceqyn.unam.edu.ar \\
\hline \hline
\end{tabular}

Division/Institute

Facultad de Ciencias Exactas, Químicas y Naturales

Division/Institute

Félix de Azara 174

ZIP code

N3300LQH City

Name

Firstname

Posadas- Misiones Country

Argentinien

\begin{tabular}{|l|l|l|l|l|} 
& \multicolumn{1}{l}{ Firstname } & Mr./Mrs./Ms. Acad. title & Phone \\
\hline Sosa & Eduardo Omar & Mr. & Prof. & $+54-3752-435099$ \\
\hline
\end{tabular}

E-Mail

Fax

es@fceqyn.unam.edu.ar 
Application Form:

Bilateral Cooperation in Education and Research

PKZ:
International Bureau of the Federal Ministry of Education and Research

Summary* (in German)

Im Rahmen des europäischen Projekts WISEBED baut ein Konsortium unter Führung des Instituts für Telematik ein europaweit verteiltes drahtloses Sensornetzwerk auf, das bereits in der Startphase aus mehr als 2000 Knoten bestehen wird. Drahtlose Sensornetze sind eine der Kerntechnologien des Future Internet; sie stellen sozusagen die Erweiterung des Internets in die reale Welt dar, indem sie es gestatten, reale Daten an jedem Ort der Welt zu erfassen und über das Internet jedem Interessenten zugänglich zu machen. Im Rahmen des hier beschriebenen Projektes soll ein weiteres Sensornetz in Argentinien aufgebaut und in das europäische WISEBED integriert werden. Das Netzwerk soll in einem Nationalpark in der Provinz Misiones installiert werden und reale Daten aus diesem Nationalpark weltweit verfügbar machen. Deutschland bzw. Europa wird profitieren, indem das weltweite Sensornetz ausgebaut wird, während Argentinien vor allem Zugriff auf die Sensornetztechnologie erhält und neue Konzepte für den Schutz der Nationalparks entwickeln kann. In diesem Programm werden vor allem die Mittel für die Reisen deutscher Wissenschaftler nach Argentinien und umgekehrt beantragt.

Project ID: 1475 
Application Form:

Bilateral Cooperation in Education and Research

PKZ:
International Bureau

of the Federal Ministry

of Education and Research

\section{Financing}

\section{German Partners}

Details of funds employed

\begin{tabular}{|l|l|r|}
\hline German Partners & Source Name & Sum (in EUR) \\
\hline Universität zu Lübeck & Institutional Budget & $27.000,00$ \\
\hline
\end{tabular}

This proposal is submitted to

International Bureau

\section{Foreign Partners}

Details of funds employed

\begin{tabular}{|l|l|r|}
\hline Foreign Partners & Source Name & Sum (in EUR) \\
\hline Universidad Nacional de La Plata & Institutional Budget & $26.500,00$ \\
\hline Universidad de Misiones & Institutional Budget & $4.800,00$ \\
\hline
\end{tabular}

In the partner country of Germany this proposal is submitted to 
Application Form:

Bilateral Cooperation in Education and Research

PKZ:
International Bureau of the Federal Ministry of Education and Research

\section{Funding requested Year 2009}

Exchange of scientists and experts (costs for stays and travel will be calculated by IB-fixed terms)

German experts visiting foreign project partners

\begin{tabular}{|l|l|r|}
\hline City/Country & Name & Duration (days) \\
\hline Argentina & Fischer, Stefan, Prof. Dr. & 14 \\
\hline Argentina & Pfisterer, Dennis, Dr. & 14 \\
\hline
\end{tabular}

Foreign experts visiting german project partners

\begin{tabular}{|l|l|r|}
\hline Destination & Name/Home Country & Duration (days) \\
\hline Germany & Diaz, Javier, Prof. (Argentina) & 15 \\
\hline Germany & Marrone, Luis, Prof. (Argentina) & 15 \\
\hline Germany & Sosa, Eduardo, Prof. (Argentina) & 30 \\
\hline
\end{tabular}

\section{Physical resources (e.g. consumables)}

\begin{tabular}{|l|l|r|}
\hline No. & Description & Estimated Cost EUR \\
\hline 1 & 15 wireless sensor nodes plus sensor module equipment & 5.000 \\
\hline
\end{tabular}

Project ID: 1475

EWA Version 1.0 CVS070522

Internationales Büro des BMBF

Königswinterer Straße 522 - 524

D-53227 Bonn
Telefon: $+49(0) 2283821-453$

Telefax: +49 (0)228 3821-444

Internet: http://www.internationales-buero.de

E-Mail: ib@dlr.de 
Application Form:

Bilateral Cooperation in Education and Research

PKZ:
International Bureau of the Federal Ministry of Education and Research

\section{Funding requested Year 2010}

Exchange of scientists and experts (costs for stays and travel will be calculated by IB-fixed terms)

German experts visiting foreign project partners

\begin{tabular}{|l|l|r|}
\hline City/Country & Name & Duration (days) \\
\hline Argentina & Fischer, Stefan, Prof. Dr. & 14 \\
\hline Argentina & Pfisterer, Dennis, Dr. & 14 \\
\hline
\end{tabular}

\section{Foreign experts visiting german project partners}

\begin{tabular}{|l|l|r|}
\hline Destination & Name/Home Country & Duration (days) \\
\hline Germany & Diaz, Javier, Prof. (Argentina) & 15 \\
\hline Germany & Marrone, Luis, Prof. (Argentina) & 15 \\
\hline Germany & Sosa, Eduardo, Prof. (Argentina) & 30 \\
\hline
\end{tabular}

\section{Cost of events}

\begin{tabular}{|l|l|r|}
\hline No. & Description & Estimated Cost EUR \\
\hline 1 & Dissemination Event & 6.000 \\
\hline \multicolumn{2}{r}{} & Sum: \\
\cline { 2 - 3 }
\end{tabular}

Project ID: 1475

EWA Version 1.0 CVS070522

Internationales Büro des BMBF

Königswinterer Straße 522 - 524

D-53227 Bonn
Telefon: $+49(0) 2283821-453$

Telefax: +49 (0)228 3821-444

Internet: http://www.internationales-buero.de

E-Mail: ib@dlr.de 
Application Form:

Bilateral Cooperation in Education and Research

PKZ:
International Bureau of the Federal Ministry of Education and Research

\section{Exchange of scientists and experts}

a) German experts visiting foreign project partners

b) Foreign experts visiting german project partners

\section{Cost of events}

Physical resources

Other Costs

Salaries

Sum

\begin{tabular}{|c|c|}
\multicolumn{1}{c|}{ Count } & Duration (days) \\
\hline 4 & 56 \\
\hline 6 & 120 \\
\hline
\end{tabular}

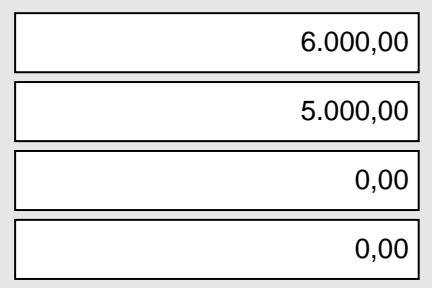

$11.000,00$

I certify, that the information contained in this application is true and correct. The realisation of the project is assured.

I agree that the basic data (incl. summary) and participating institutions can be published.

Signature of the principal investigator

The project proposal was elaborated jointly

German Partners

Date

Name

Signature

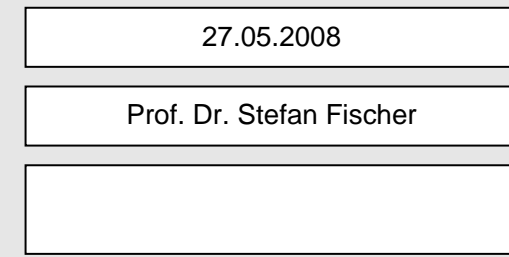

Foreign Partners

Date

Name

Signature

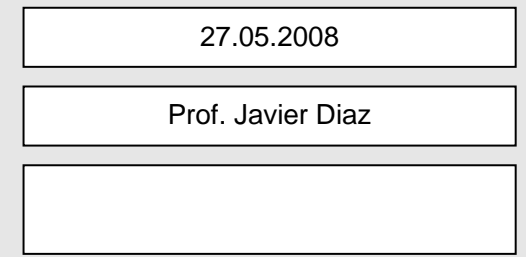

\section{Signature of institution or company}

\section{German partner}

Date

Name

Signature

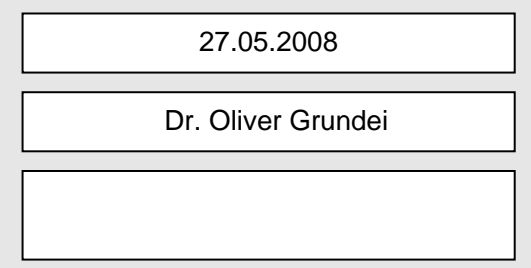

Foreign partner

Date

Name

Signature
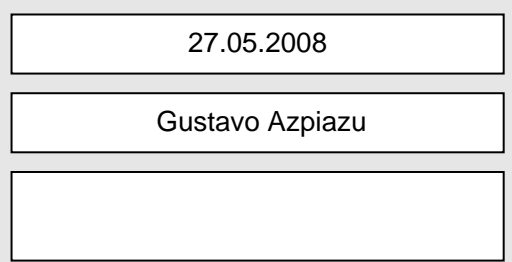

Project ID: 1475

Internationales Büro des BMBF

Königswinterer Straße 522 - 524

D-53227 Bonn
Telefon: $+49(0) 2283821-453$

Telefax: $+49(0) 2283821-444$

Internet: http://www.internationales-buero.de

E-Mail: ib@dlr.de 


\section{Project Description}

Scientific objectives (including relevance to the topics of BMBF, EU or other funding programmes if applicable)

In this project the partners intend to set up a wireless sensor network in Argentina, specifically in the Reserve Biosphere Yaboty. The new network will then be connected to the European network of sensor networks WISEBED which will be established from June 2008 on, and it will deliver real-world data concerning the environment in these parks to everybody in the world who is interested. A second network might be established close to the Ocean coast of the Province of Buenos Aires near the UNLP. Besides the training and expertise provided, the project will be incorporated in the regular courses of the Magister on Data Networks in the UNLP and also will produce several post-graduate thesis. WISEBED is part of the European Future Internet strategy, which is also broadly discussed on the Germany national level (see www.future-internet.org, for instance, for an initiative backed by the German Gesellschaft für Informatik). An important aspect of Future Internet is the seamless inclusion of wireless sensor networks in order to make access to all kinds of real-world data possible for everybody over the Internet. As such, this project is a perfect extension to WISEBED.

\section{Project description: nature and extent of the intended project cooperation}

In this project, three partners will cooperate in order to set up this network and connect it to WISEBED: on the Germany side, it is the Institute of Telematics at the University of Lübeck, which also leads the WISEBED consortium. On the Argentinean side, it's the Universidad Nacional de La Plata, which has the capability of being counterpart of the European project, because of its academic level and the people involved. The Universidad Nacional de Misiones, a very young university, because of its location, is a necessary partner in order to provide the backup for the success of the endeavour. It is necessary to mention the commitment of the Government of the Province Misiones in Argentina, which monitors the provincial parks, and has agreed to provide the authorization for the setup of the net. We think that all this work would provide significant result to the area from the theoretical and operative point of view. They would also promote many graduate and postgraduate courses and activities alike not so common in the actual curricula of all universities that will participate in the project.

\section{Methods}

Mark Weiser, the inventor of the term "Ubiquitous Computing" (wireless sensor networks are an enabling technology for ubiq. computing), was convinced that "The research method for ubiquitous computing is standard experimental computer science: the construction of working prototypes of the necessaryinfrastructure in sufficient quantity to debug the viability of the systems in everyday use, using ourselvesand a few colleagues as guinea pigs." This is exactly the method we are following in this project: we are creating sensor network prototypes to both learn something about the practical implications of this technology and to advance the field of ubiquitous computing in combination with the Future Internet. Apart from that, an important aspect of this project is transfer of knowledge which we implement both by traditional courses in a university environment and by hands-on training in order to enable people to quickly set up successful sensor network prototypes.

Project ID: 1475 
Application Form:

Bilateral Cooperation in Education and Research
International Bureau of the Federal Ministry of Education and Research

PKZ:

LpSys:

\section{Comprehensive Workplan}

Work packages

WP 1: Transfer of Sensor Network Knowledge to the Argentinean partners. Lübeck will execute remote instruction and face-to-face courses with the Argentinean partners in order to set grounds for successful practical project work. WP 2: Setting up the sensor network and creation of an application Here, the Argentinian partners will set up the network and program an application suitable for the selected national park. WP 3: Connecting the sensor network to WISEBED The University of Lübeck will provide the necessary middleware software which will by then be available within WISEBED The Argentinean partners will help in setting up the software on the local site. WP 4: Operate the network: We expect that the network is more or less self-organized (as desirable for sensor networks), but there is probably still some need for regular check-ups. WP 5: Dissemination of results All partners will present their results in a general assembly meeting of the WISEBED project. Second, there will be a workshop in the Universidad de La Plata as well as one in Misiones where the results will be presented publically.

\section{Distribution of work packages (if more than one partner)}

WP 1: University of Lübeck WP 2: Misiones and La Plata, potentially with support from Lübeck when necessary WP 3: University of Lübeck WP 4: Misiones WP 5: all partners

\section{Timetable}

WP 1: $01 / 2009$ to $03 / 2009 \quad$ WP 2: 04/2009 to $12 / 2009 \quad$ WP 3: $01 / 2010$ to $03 / 2010 \quad$ WP 4: $01 / 2010$ to 12/2010 WP 5: $01 / 2010$ to $12 / 2010$

Project ID: 1475

EWA Version 1.0 cvs070522

Internationales Büro des BMBF

Königswinterer Straße 522 - 524

D-53227 Bonn
Telefon: $+49(0) 2283821-453$

Telefax: +49 (0)228 3821-444

Internet: http://www.internationales-buero.de

E-Mail: ib@dlr.de 


\section{Reasons for co-operation}

Previous contacts, impulses

The idea for this project resulted from a research visit of Eduardo Sosa from Universidad de Misiones at the University of Lübeck in the summer of 2007. During this 2-month-stay, the two partners developed the principle concept for such a project and then decided to look for additional partners and frameworks in which the project could be realized. In Argentina, Javier Diaz from UNLP was asked to join in. In Germany, meanwhile, University of Lübeck set up the WISEBED consortium and applied for European funding which was granted in the beginning of 2008. WISEBED will start in June 2008, so it would be an excellent timing to take the first non-European partner into the project from 2009 on.

\section{Benefits for each country}

By cooperating with a non-European partner, Germany/Europe will be able to extend its Future Internet strategy to other parts of the world and thus challenge the US leadership in this area. Additionally, German technology (the sensor nodes and the sensor network algorithms) will be exported to external countries; as a result, Germany will improve its position with respect to competitive advantages in this field. Finally, European researchers will profit a lot from the extension of WISEBED in terms of size, but also because they get access to real-world data - which is not so common in today's sensor net projects. Argentina will profit because they get access to European high-tech information technology and the knowledge developed in Europe. They will learn from German researchers about the technologies in the rapidly emerging field of wireless sensor networks, and they will get to use real sensor network technology. Also, Argentina will be able to test new technology for monitoring and protecting their national resources, especially (in this project) in oxygen reserves of the world, like the biosphere Yaboty.

\section{Intended follow-up incl. possible sources for further fin. support, utilization of results}

Wireless sensor networks are considered to be one of the key technologies in information technology in the coming years. It is the goal of the project WISEBED - and thus also of this associated project - to clear the path for a broad usage of such networks. Projects like this one intend to show the possibilities that wireless sensor networks offer to a broad range of industries. By show-casing the project results, we expect to create major interest in in several branches of both European and Argentinian economy. 


\section{Qualifications of the applicant}

Experience of participating institutions

The Institute of Telematics (ITM, www.itm.uni-luebeck.de) is one of Germany's leading institutions concerning wireless sensor networks and their real-world implementation. It has conducted a number of such large-scale deployments such as the in the projects MarathonNet and FlegSenS. ITM published about 20 papers/year over the last 4 years. Due to its experiences, ITM has been selected to head the WISEBED consortium.LINTI (www.linti.unlp.edu.ar), located in La Plata, focuses on education in Networks, Security and Software Development and applications for our society. In Networks, LINTI has expertise ranging from gigabit to mobile ad-hoc networks and hence, sensor networks are an ideal candidate to establish academic relationsships deepenig this expertise in Argentina. Technology and natural science are the basis for Universidad de Misiones' (UNaM, www.unam.edu.ar) activities. UNaM fosters the development of basic technology competence in this region of Argentina's northeast. UNaM has direct access to the site of the network to be built.

\section{References (Relevant publications, Patenets etc.)}

[1200 characters are a hevay restriction here!]The University of Lübeck, Institute for Telematics, has published numerous papers on wireless sensor networks. Due to space restrictions, we can only point to the web site: http://www.itm.uni-luebeck.de/publications/ UNLP is active in Internet and mobile networking technologies, which are both important for this project. Two examples: Evaluating the Usability of the Mobile Interface of an Educational Website" Javier diaz, Ivana Harari y Paola Amadeo; CISSE 2007 Proceedings of the International Joint Conferences on Computer, Information, and Systems Sciences, and Engineering. Diciembre 2007 Movilidad en IPv4, Javier Díaz, Mauricio Demasi, Matías Robles, Germán Vodopivec, CACIC 2006, Universidad Nacional de San Luis, Argentina. ISBN:950-609-050-5

\section{Supporting young Scientists}

\section{Participation of young scientists}

On the German side, the project itself will mainly be executed by Prof. Fischer and Dr. Pfisterer. However, due to the close cooperation with the WISEBED project, at least two PhD students will be in close contact with this project and provide input whenever suitable. In addition, master theses will be excuted in the context of this project.On the Argentinean side, we think that all this work would provide significant result to the area from the theoretical and operative point of view. They would also promote many graduate and postgraduate courses and activities alike not so common in the actual curricula of all universities that will participate in the project. 
Application Form:

Bilateral Cooperation in Education and Research

PKZ:

LpSys:
International Bureau

of the Federal Ministry

of Education and Research

Funding requested Year

2009

Justification for Cost of Events

Project ID: 1475

EWA Version 1.0 CVS070522

Internationales Büro des BMBF

Königswinterer Straße 522 - 524

D-53227 Bonn
Telefon: $+49(0) 2283821-453$

Telefax: +49 (0)228 3821-444

Internet: http://www.internationales-buero.de

E-Mail: ib@dlr.de 
PKZ:

LpSys:

\section{Funding requested Year}

\section{0}

\section{Justification for Cost of Events}

Justification for No. 1

The results of the project should be presented to a broad public in Argentina. There will be one event in La Plata and one at the the Universidad de Misiones within a few days time. 
Application Form:

Bilateral Cooperation in Education and Research
International Bureau of the Federal Ministry of Education and Research

PKZ:

LpSys:

\section{Funding requested Year}

\section{9}

\section{Justification for Physical Resources}

Justification for No. 1

The Argentinean partner does not have a sensor network physically available yet; with this investment, a basic network can be set up which will be extended by own funds of the partner (Universidad de Misiones has just applied to the provincial authorities, for 45 more nodes). 
Application Form:

Bilateral Cooperation in Education and Research

PKZ:

LpSys:
International Bureau

of the Federal Ministry

of Education and Research

Funding requested Year

\section{0}

Justification for Physical Resources

Project ID: 1475

EWA Version 1.0 cvs070522

Internationales Büro des BMBF

Königswinterer Straße 522 - 524

D-53227 Bonn
Telefon: $+49(0) 2283821-453$

Telefax: +49 (0)228 3821-444

Internet: http://www.internationales-buero.de

E-Mail: ib@dlr.de 
Application Form:

Bilateral Cooperation in Education and Research

PKZ:

LpSys:
International Bureau

of the Federal Ministry

of Education and Research

Funding requested Year

2009

Justification for Other Costs

Project ID: 1475

EWA Version 1.0 cvs070522

Internationales Büro des BMBF

Königswinterer Straße 522 - 524

D-53227 Bonn
Telefon: $+49(0) 2283821-453$

Telefax: $+49(0) 2283821-444$

Internet: http://www.internationales-buero.de

E-Mail: ib@dlr.de 
Application Form:

Bilateral Cooperation in Education and Research

PKZ:

LpSys:
International Bureau

of the Federal Ministry

of Education and Research

Funding requested Year

2010

Justification for Other Costs

Project ID: 1475

EWA Version 1.0 CVS070522

Internationales Büro des BMBF

Königswinterer Straße 522 - 524

D-53227 Bonn
Telefon: $+49(0) 2283821-453$

Telefax: +49 (0)228 3821-444

Internet: http://www.internationales-buero.de

E-Mail: ib@dlr.de 


\section{Apéndice F: Especificaciones}

\section{Físicas y Mecánicas de los nodos utilizados}

Source: http://www.coalesenses.com/index.php?page=products

\section{Core Module}

\begin{tabular}{|l|r|}
\hline Processor \\
\hline RAM $^{1}$ & $128 \mathrm{kB}$ \\
\hline Serial Flash & \\
\hline Current draw operation & $512 \mathrm{kB}$ \\
\hline Current draw sleep mode & $\sim 6 \mathrm{~mA}$ \\
\hline RF Transceiver & $\sim 3 \mu \mathrm{A}$ \\
\hline Frequency & $2.4 \mathrm{GHz}$ \\
\hline Bandwidth & $250 \mathrm{kbit} / \mathrm{s}$ \\
\hline channels & 16 \\
\hline Transmit power & $2.5 \mathrm{~dB}$ \\
\hline Receive sensitivity & $-95 \mathrm{~dB}$ \\
\hline Current consumption & $\sim 16 \mathrm{~mA}$ \\
\hline Electromechanical & \\
\hline Supply voltage & \\
\hline Dimensions & $2.0 \mathrm{~V}-5.5 \mathrm{~V}$ \\
\hline Weight & $45 \mathrm{~mm} \times 30 \mathrm{~mm}$ \\
\hline Temperature range & $10 \mathrm{~g}$ \\
\hline
\end{tabular}

1) Shared for program and data

2) With voltage regulator in use 


\section{Gateway Module}

\begin{tabular}{|lr|}
\hline Gateway Module & \\
Maximum baud rate & $115200 \mathrm{~kb} / \mathrm{s}$ \\
Max. supply current (USB) & $500 \mathrm{~mA}$ \\
Current draw operation & tbd \\
Electromechanical & \\
USB/RS232 cable length & $\sim 800 \mathrm{~mm}$ \\
Dimensions & $45 \times 34 \mathrm{~mm}$ \\
Weight & $6 \mathrm{~g}$ \\
Temperature range & -20 to $70{ }^{\circ} \mathrm{C}$ \\
\hline
\end{tabular}

\section{Security Sensor Modules}

\begin{tabular}{|l|r|}
\hline Accelerometer & $\pm 2 \mathrm{~g}$ or $\pm 6 \mathrm{~g}$ \\
\hline Range & $40 \mathrm{~Hz}$ or $640 \mathrm{~Hz}$ \\
\hline Frequency & $\sim 650 \mu \mathrm{A}$ \\
\hline Current draw operation & $\sim 1 \mu \mathrm{A}$ \\
\hline Current draw sleep mode & $\sim 10 \mathrm{~m}$ \\
\hline Passive Infrared Sensor & $93 \% 110^{\circ}$ \\
\hline Range & $\sim 300 \mu \mathrm{A}$ \\
\hline Angle (hor:/vert.) & $0 \mu \mathrm{\Lambda}$ \\
\hline Current draw operation & \\
\hline Current draw sleep mode & $3.3 \mathrm{~V}$ \\
\hline Electromechanical & \multicolumn{2}{|c|}{} \\
\hline Supply voltage & $8 \mathrm{~g}$ \\
\hline Dimensions & -20 to $70^{\circ} \mathrm{C}$ \\
\hline Weight & \\
\hline Temperature range &
\end{tabular}




\section{Weather Sensor Module}

\begin{tabular}{|l|r|}
\hline Temperature and relative humidity \\
\hline Accuracy $(\mathrm{t} / \mathrm{rh})$ & $1{ }^{\circ} \mathrm{C} / 3 \%$ \\
\hline Resolution $(\mathrm{t} / \mathrm{rh})$ & $0.1^{\circ} \mathrm{C} / 0.1 \%$ \\
\hline Range $(\mathrm{t} / \mathrm{rh})$ & $-20 . .+70^{\circ} \mathrm{C} / 0.100 \%$ \\
\hline Current draw operation & $\sim 800 \mu \mathrm{A}$ \\
\hline Current draw standby & $\sim 0.5 \mu \mathrm{A}$ \\
\hline Barometric pressure & \\
\hline Range & $10 . .1100 \mathrm{mbar}$ \\
\hline Resolution & $0.1 \mathrm{mbar}$ \\
\hline Accuracy & $1.5 \mathrm{mbar}$ \\
\hline Frequency & $1 \mathrm{~Hz}$ \\
\hline Current draw operation & $\sim 1 \mathrm{~mA}$ \\
\hline Current draw standby & $<0.1 \mu \mathrm{A}$ \\
\hline Electromechanical & \\
\hline Supply voltage & $2.4 .3 .6 \mathrm{~V}$ \\
\hline Dimensions & $38 \mathrm{~mm} \times 30 \mathrm{~mm}$ \\
\hline Weight & $6 \mathrm{~g}$ \\
\hline Temperature range & $-20 . .+70^{\circ} \mathrm{C}$ \\
\hline
\end{tabular}




\section{Solar Power Harvesting System}

\begin{tabular}{|c|c|}
\hline \multicolumn{2}{|c|}{ Power Manage ment Module } \\
\hline Standby Current & $75 \mu \mathrm{A}$ \\
\hline Input Voltage & 4.35 to $12 \mathrm{~V}$ \\
\hline Output Voltage & 2.5 to $5.0 \mathrm{~V}$ \\
\hline Weight & $8 \mathrm{~g}$ \\
\hline Dimensions & $30 \times 37 \mathrm{~mm}$ \\
\hline Temperature range & -20 to $70^{\circ} \mathrm{C}$ \\
\hline \multicolumn{2}{|l|}{ Housing } \\
\hline Dimensions & $81 \times 82 \times 87 \mathrm{~mm}$ \\
\hline Protection Level & IP 66 \\
\hline Weight & $206 \mathrm{~g}$ \\
\hline \multicolumn{2}{|l|}{ Battery } \\
\hline Nominal Voltage & $3.7 \mathrm{~V}$ \\
\hline Nominal Capacity & $6750 \mathrm{mAh}$ \\
\hline Cable Length & $\sim 45 \mathrm{~mm}$ \\
\hline Dimensions & $67 \mathrm{~m} 59 \times 19 \mathrm{~mm}$ \\
\hline Weight & $136 \mathrm{~g}$ \\
\hline Charge temperature & 2 to $44^{\circ} \mathrm{C}$ \\
\hline Discharge temperature & -25 to $70^{\circ} \mathrm{C}$ \\
\hline \multicolumn{2}{|l|}{ Solar Panel } \\
\hline Nominal Power & $1.6 \mathrm{~W}$ \\
\hline Current at MPP & $250 \mathrm{~mA}$ \\
\hline Voltage at MPP & $6 \mathrm{~V}$ \\
\hline Open Circuit Voltage & $8 \mathrm{~V}$ \\
\hline Cable Length & $\sim 0.9 \mathrm{~m}$ \\
\hline Dimensions & $100 \times 240 \mathrm{~mm}$ \\
\hline Weight & $460 \mathrm{~g}$ \\
\hline Temperature range & -40 to $85^{\circ} \mathrm{C}$ \\
\hline
\end{tabular}




\section{Environmental Sensor Module}

\begin{tabular}{|l|r|}
\hline Thermometer \\
\hline Sensitivity & $1{ }^{\circ} \mathrm{C}$ \\
\hline Frequency & $10 \mathrm{~Hz}$ \\
\hline Range & -55 to $125^{\circ} \mathrm{C}$ \\
\hline Current draw operation & $\sim 250 \mu \mathrm{A}$ \\
\hline Current draw sleep mode & $\sim 1 \mu \mathrm{A}$ \\
\hline Light Sensor & \multicolumn{2}{|r|}{$1 \mathrm{tbd}$} \\
\hline Range & $1 \mathrm{~Hz}$ \\
\hline Frequency & $\sim 35 \mu \mathrm{A}$ \\
\hline Current draw operation & $\sim 10 \mu \mathrm{A}$ \\
\hline Current draw sleep mode & \\
\hline Electromechanical & $3.3 \mathrm{~V}$ \\
\hline Supply voltage & $45 \mathrm{~mm} \times 30 \mathrm{~mm}$ \\
\hline Dimensions & \\
\hline Weight & -20 to $70^{\circ} \mathrm{C}$ \\
\hline Temperature range &
\end{tabular}

\section{AA Battery holder}

\begin{tabular}{|l|r|}
\hline Battery Holder \\
\hline Battery Type & $2 \times$ AA \\
\hline Dimensions & $68 \times 33 \times 19 \mathrm{~mm}$ \\
\hline Connector cable length & $60 \mathrm{~mm}$ \\
\hline Weight & $\sim 10 \mathrm{~g}$ \\
\hline Temperature range & -20 to $70^{\circ} \mathrm{C}$ \\
\hline
\end{tabular}

